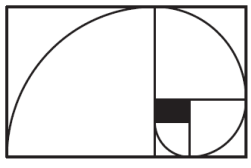

\title{
Dynamics and global geometry of manifolds without conjugate points
}

\section{Rafael O. Ruggiero}

\begin{abstract}
Manifolds with no conjugate points are natural generalizations of manifolds with nonpositive sectional curvatures. They have in common the fact that geodesics are global minimizers, a variational property of geodesics that is quite special. The restriction on the sign of the sectional curvatures of the manifold leads to a deep knowledge about the topology and the global geometry of the manifold, like the characterization of higher rank, nonpositively curved spaces as symmetric spaces. However, if we drop the assumptions concerning the local geometry of the manifold the study of geodesics becomes much harder. The purpose of this survey is to give an overview of the classical theory of manifolds without conjugate points where no assumptions are made on the sign of the sectional curvatures, since the famous work of Morse about minimizing geodesics of surfaces and the works of Hopf about tori without conjugate points. We shall show important classical and recent applications of many tools of Riemannian geometry, topological dynamics, geometric group theory and topology to study the geodesic flow of manifolds without conjugate points and its connections with the global geometry of the manifold. Such applications roughly show that manifolds without conjugate points are in many respects close to manifolds with nonpositive curvature from the topological point of view.
\end{abstract}





\section{Contents}

$\begin{array}{ll}\text { Introduction } & 7\end{array}$

1 Preliminaries on Riemannian geometry and geodesics 11

1 Riemannian manifolds as metric spaces . . . . . . . . . . . . 11

2 The Levi-Civita connection . . . . . . . . . . . . . . . . 12

3 The differential equation of geodesics . . . . . . . . . . . . 13

4 The exponential map, curvature and Jacobi fields . . . . . . 14

5 The geodesic flow . . . . . . . . . . . . . 17

5.1 Sasaki metric, Jacobi fields and the differential of the geodesic flow . . . . . . . . . . . . 18

5.2 Transversal symplectic structure of the geodesic flow 20

6 The universal covering . . . . . . . . . . . . . . . . . . . 24

6.1 Homotopy classes . . . . . . . . . . . . . . 25

6.2 Covering transformations and fundamental group . . 26

6.3 Covering transformations as isometries . . . . . . 27

2 Dynamical systems and stability 28

1 Limit set and non-wandering set . . . . . . . . . . . . . 28

2 Hyperbolic sets and Anosov dynamics . . . . . . . . . . 30

3 Local product structure, expansiveness and stability . . . . 34

4 The stability conjecture(s) . . . . . . . . . . . . 45

$3 C^{1}$ tools to study geometry and dynamics: Jacobi fields, $\begin{array}{ll}\text { hyperbolicity and global geometry } & 48\end{array}$

1 Negative curvature and Anosov dynamics . . . . . . . . . . 49

1.1 Rauch's comparison theorem . . . . . . . . . . 49

1.2 Asymptotic Jacobi fields and Riccati equation . . . . 50

1.3 Green subbundles .............. 53

1.4 Proof of Anosov's theorem and the impact of hyperbolicity in the global geometry of geodesics . . . . . 54

2 Characterization of Anosov geodesic flows in manifolds without conjugate points . . . . . . . . . . . 55 
2.1 On the divergence of Jacobi fields in manifolds without conjugate points . . . . . . . . . . . . . 56

Quasi-Anosov systems . . . . . . . . . . . 58

2.3 Quasi-convexity of Jacobi fields and continuity of Green subbundles . . . . . . . . . . . . . . . . . 59

2.4 Hyperbolic behavior of Green subbundles . . . . . . 60

3 Anosov dynamics and globally minimizing properties of geodesics: hyperbolicity implies the absence of conjugate points . . . 63

3.1 Conclusions . . . . . . . . . . . . . 65

4 Further applications of the Riccati equation in ergodic theory 66

$4 C^{0}$ tools to study global geometry of geodesics in the universal covering of manifolds without conjugate points $\quad 69$

1 Horospheres and Busemann flows in $\tilde{M}$. . . . . . . . 70

2 Two-dimensional tori without conjugate points are flat . . . . . . . . . . . . . 75

3 Surfaces without conjugate points of higher genus, quasigeodesics and quasi-convexity . . . . . . . . . . . 77

4 Divergence of geodesic rays and continuity of $H_{\theta}(0)$ with respect to $\theta \ldots \ldots \ldots \ldots$. . . . . . . . . . . . 84

4.1 Asymptoticity and quasi convexity . . . . . . . 84

4.2 Divergence of geodesic rays in surfaces without conjugate points . . . . . . . . . . 86

4.3 Divergence of geodesic rays in higher dimensions . . 89

4.4 The continuity of horospheres . . . . . . . . . . 90

5 Summary of results about the geodesic flow of compact surfaces without conjugate points

6 Higher dimensional manifolds without conjugate points: Convexity and flats in the universal covering . . . . . . . . . 93

5 Expansive geodesic flows in manifolds without conjugate points: an example of the application of $C^{0}$ methods to study dynamics and global geometry

1 Expansiveness and no conjugate points . . . . . . . . 96

2 Expansive geodesic flows without conjugate points in higher dimensions ................... 97

2.1 Expansiveness implies quasi-convexity and asymptoticity . . . . . . . . . . . . . . . . . . . 98

2.2 Strong stable and unstable sets . . . . . . . . . . . 100

3 Divergence of geodesic rays in $\tilde{M}$. . . . . . . . . . . . . . 104

4 Proof of Theorem 5.2 and applications . . . . . . . . . . . . 104 


\section{CONTENTS}

6 The fundamental group, global geometry and geometric $\begin{array}{ll}\text { group theory } & 108\end{array}$

1 Fundamental group, algebra and geometry . . . . . . . . . 108

2 Fundamental group in negative curvature, Preissmann's theorem and Gromov hyperbolic groups . . . . . . . . . . . . . 113

3 Preissmann property implies Gromov hyperbolicity? . . . . . . . . . . . . . . . . . . 117

4 Expansive dynamics, weak stability and Gromov hyperbolicity 120

7 Weak hyperbolicity of the geodesic flow and global hyperbolic geometry in nonpositive curvature

1 Homoclinic geodesics in the two torus and instability . . . . 131

2 The closing lemma for flat planes in analytic manifolds with nonpositive curvature and the proof of the conjecture in this

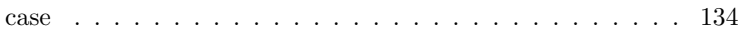

3 Sharpening the shadowing result by means of Aubry-Mather

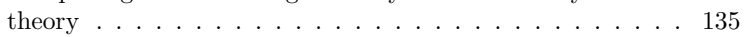

8 Weak hyperbolicity of geodesics, global geometry and Poincaré conjecture

1 Homoclinic geodesics in manifolds without conjugate points

and variable curvature sign . . . . . . . . . . . . 143

1.1 A generalization of Hedlund's tunnels . . . . . . . . 144

1.2 On finding homoclinic, minimizing geodesics in perturbed metrics . . . . . . . . . . . . . 146

1.3 Morse homoclinic geodesics and bounded asymptote . . . . . . . . . . . . . . 155

2 Bounded asymptote and Preissmann's property . . . . . . . . . . . . . . . 157

2.1 Shadowing of geodesics and the Preissmann property in bounded asymptote . . . . . . . . . 158

2.2 Topological stability and Preissmann property . . . 159

3 Further results in certain manifolds

without conjugate points . . . . . . . . . . . . 160

3.1 Morse homoclinic geodesics in perturbations of metrics without conjugate points . . . . . . . . . . 161

3.2 On the nonexistence of Morse homoclinic geodesics in manifolds without conjugate points . . . . . . . 162

3.3 Proof of Theorem $8.2 \ldots \ldots \ldots$

3.4 Proof of Theorem $8.3 \ldots \ldots \ldots \ldots$

4 Weak stability and Thurston's geometrization conjecture . . 166

4.1 The Poincaré conjecture . . . . . . . . . . . 167

4.2 Thurston's geometrization conjecture . . . . . . . 167

4.3 Applications of the Thurston's geometrization conjecture to weak stability problems . . . . . . . 169 
CONTENTS

$5 \quad$ Further remarks and questions . . . . . . . . . . . 173

Bibliography 175 


\section{Introduction}

The theory of manifolds without conjugate points is one of the most challenging research areas in geometry. A complete Riemannian manifold $(M, g)$, where $M$ is a $C^{\infty}$ manifold and $g$ is a $C^{\infty}$ Riemannian metric in $M$, has no conjugate points if the exponential map at every point is non-singular. This is equivalent to the fact that every geodesic is globally minimizing in the universal covering $\tilde{M}$ endowed with the pullback of the metric $g$ by the covering map. Namely, the distance between any two points in a geodesic in $\tilde{M}$ is just the length of the subset of this geodesic bounded by these two points. Hyperbolic space and manifolds of nonpositive curvature are well known examples of such manifolds, but the question of knowing if a manifold without conjugate point admits a metric with nonpositive curvature is very difficult and open.

The absence of conjugate points is on the one hand, a strong condition from the topological point of view; but on the other hand it gives no hints about the local geometry of the manifold. So the usual way in geometry to get the global description of manifolds from local data - like in the theory of manifolds whose sectional curvatures have constant sign - does not proceed in this theory. The geometric theory of manifolds without conjugate points is therefore the result of an exciting interplay between many areas in mathematics, from classical Riemannian geometry and dynamical systems to calculus of variations, classical mechanics and geometric group theory.

The purpose of this survey is to give an overview of the main techniques and results about one of the main subjects of the theory: the relationships between the topological dynamics of the geodesic flow, the topology and the global geometry of the manifold. We shall focus on the aspects of the theory which are not part of the theory of manifolds with nonpositive curvature, where there are already fairly good, complete surveys and books (see for instance [34], [8], [5], [60]). The theory of manifolds with nonpositive curvature is much richer in results than the theory of manifolds without conjugate points, simply because the convexity of many geometric functions and objects determined by the nonpositive curvature is a powerful tool to study global geometry, topology and the dynamics of the geodesic 
flow of the manifold. This is perhaps the reason why the theory of manifolds without conjugate points is not so popular: it is much harder and the results are quite often weaker than in the theory of manifolds with nonpositive curvature.

The topics presented in the survey could be classified in two main subjects: first of all the application of smooth methods or $C^{1}$ methods to study the geometry and the dynamics of the geodesic flow in manifolds without conjugate points; and secondly the application of $C^{0}$ or variational methods to study geodesics and global geometry in such manifolds. The application of $C^{1}$ methods concerns of course Jacobi fields and curvature, and such methods allow to study global structures from local data, like in the theory of manifolds with nonpositive curvature. We shall illustrate some of these methods, unfortunately we have to choose and many interesting applications won't be considered. The variational or $C^{0}$ methods concern the use of the globally minimizing nature of geodesics in the universal covering regardless of the sign of sectional curvatures. This is in fact the only information we have for sure about manifolds without conjugate points. We shall apply this kind of methods to study the connections between weak forms of hyperbolicity in the geodesic flow and some hyperbolic aspects of the topology and the global geometry of the manifold.

The survey is divided in 8 chapters. Chapter 1 has some preliminaries of Riemannian geometry and some basic results of the theory of geodesic flows. Chapter 2 contains a survey about the theory of stability of dynamical systems which is closely related with the theory of geodesic flows in manifolds with negative curvature.

In Chapter 3 we analyze some relevant $C^{1}$ methods of the theory of manifolds without conjugate points. Chapter 3 introduces many smooth objects associated to manifolds without conjugate points, mostly related to the asymptotic behavior of Jacobi fields. Green subspaces and the Riccati differential equation associated to them are considered; we show that geodesic flows of compact manifolds with negative curvature are Anosov and we show the characterization of Anosov flows in manifolds without conjugate points: they are characterized by the linear independence of Green subspaces. The theory of the Riccati equation is closely related with the theory of Lagrangian invariant graphs which are important tools in calculus of variations (Hamilton-Jacobi equation, Aubry-Mather theory). These ideas gave rise to a different, elegant proof of the famous Klingengerg's theorem, made by Mañé, who went further and showed that the existence of a continuous invariant, Lagrangian subbundle in the unit tangent bundle of a compact manifold implies the absence of conjugate points. We won't prove this theorem in the survey, the book by Paternain [72] is a very good reference for the subject. We chose the above three theorems as examples of the 
applications of $C^{1}$ methods because they were turning points in the theory of Anosov geodesic flows. Anosov's theorem shows the stability of geodesic flows in negative curvature; Eberlein's theorem gives a beautiful geometric characterization of Anosov geodesic flows in terms of invariant subspaces naturally associated to manifolds without conjugate points. And Klingenberg's theorem shows an important link between hyperbolic dynamics and manifolds without conjugate points through the calculus of variations. Moreover, Green subspaces are Lagrangian, and Mañé's theorem suggests that the existence of "many" Lagrangian, invariant subspaces is a typical feature of manifolds without conjugate points.

In Chapter 4 we start with the study of $C^{0}$ methods of the theory of manifolds without conjugate points. We start by the description of surfaces without conjugate points. We show the famous flat torus Hopf's theorem; we show the striking Morse's description of globally minimizing geodesics of surfaces of higher genus, and we prove Green's result about the divergence of geodesic rays of surfaces without conjugate points. Then we define all the usual geometric objects of the theory: horospheres, Busemann functions, and we give a summary of results of what is known about their regularity.

The remaining chapters are devoted to study the interplay between the dynamics of "weakly stable" geodesic flows without conjugate points and the global geometry of the manifold. Roughly speaking, weak stability will refer to different notions of stability - already discussed in Chapter 2 - which are weaker than structural stability. We shall expose a theory developed since 1990 in several papers by R. Ruggiero ([83], [84], [85], [86], [87], [88], [89], [90], [91], [92]), based in the following main idea: although weak forms of stability might not be enough to give accurate data about local geometry, they are strong enough to imply many important properties of global hyperbolic geometry.

With the basic topological tools introduced in Chapter 4, we start in Chapter 5 the application of $C^{0}$ methods to study the dynamics of expansive geodesic flows in compact manifolds without conjugate points. We show that such flows have practically the same topological dynamics of an Anosov geodesic flow. Here we find an example of how powerful is the combination of the globally minimizing nature of geodesics and a topological assumption of hyperbolic nature (expansiveness) on the geodesic flow. Either one of these conditions considered independently seems to be very weak. The results of this chapter are very important for the sequel, they motivate many questions about the relationship between global geometry and topological hyperbolic dynamics.

In Chapter 6 we pursue the study of the global geometry of the manifold by looking at the relationships between the fundamental group and 
the geodesic flow. At this point we give a brief introduction of geometric group theory, which showed to be very convenient and enlightening in the study of global geometry. We define Gromov hyperbolic groups, Preissmann groups, and many geometric objects associated to groups as metric spaces. We apply such notions to describe the fundamental group of compact manifolds without conjugate points and expansive geodesic flow: expansiveness implies Gromov hyperbolicity. This surprising fact strengthens the analogies between Anosov and expansive geodesic flows in what concerns the global geometry of the manifold. And motivate us to look for the weakest hyperbolic-like assumptions on the geodesic flow which still grant some hyperbolicity for the fundamental group. One interesting question arising in this context is a sort of topological version of the stability conjecture: does topological stability of the geodesic flow in compact manifolds without conjugate points imply Gromov hyperbolicity?

In the final two chapters we are devoted to explain the main ideas and results concerning the interplay between weak stability properties of the geodesic flow in manifolds without conjugate points, the global geometry of the universal covering, and geometric group theory. We make first a review of results in nonpositive curvature in Chapter 7 and then in Chapter 8 we present some recent developments for manifolds without conjugate points and variable curvature sign. We would like to point out that some of the main ideas of this theory have the flavor of the theory of connecting orbits in Aubry-Mather theory. We conclude the survey with a combination of the results of Chapters 7 and 8 and the recent work of Perelmann about the solution of the Poincaré conjecture. We get the existence of metrics of negative curvature in some three dimensional manifolds without conjugate points and weakly hyperbolic geodesic flows, and we could conjecture that almost every three dimensional, compact manifold without conjugate points and expansive geodesic flow admits a metric of negative curvature.

I am deeply grateful to Professor Nalini Anantharaman, who made a careful revision of the manuscript, found many mistakes in its preliminary versions and gave valuable suggestions and comments which improved substantially the reading of the text. 


\section{Chapter 1}

\section{Preliminaries on Riemannian geometry and geodesics}

Our main references here are [29], [34], [31], however any basic text of Riemannian manifolds would contain the material of the section. We shall use the notation $(M, g)$ to designate a $C^{\infty}$ Riemannian manifold: a differentiable manifold endowed with a Riemannian metric $g$ that will be assumed to be also $C^{\infty}$.

\section{Riemannian manifolds as metric spaces}

Let $T M$ be the tangent space of $M$. We shall call by $l_{g}$ the length associated to the metric $g$, i.e., given a differentiable curve $\gamma:[a, b] \longrightarrow M$, its lenght in the metric $g$ is

$$
l_{g}(\gamma)=\int_{a}^{b}\left\|\gamma^{\prime}(t)\right\| d t
$$

where $\left\|\gamma^{\prime}(t)\right\|=\sqrt{g\left(\gamma^{\prime}(t), \gamma^{\prime}(t)\right)}$ is the norm of $\gamma^{\prime}(t)$ in the metric $g$. The notion of length can be defined in fact in the family of rectifiable curves, or curves with bounded variation. There is a natural distance associated to the Riemannian metric $g$ : if $x, y$ are points in $M$, then

$$
d(x, y)=\inf \left\{l_{g}(\gamma), \gamma:[0,1] \rightarrow M, \gamma(0)=x, \gamma(1)=y\right\}
$$

where $\gamma$ is a differentiable (rectifiable) curve. The intuitive idea of a geodesic joining $x$ and $y$ corresponds to a curve joining these points whose length attains the distance $d(x, y)$. The Riemannian manifold $(M, g)$ is endowed with a natural structure of metric space associated to this distance, and we say that $(M, g)$ is complete if it is complete as a metric space.

This idea goes back to ancient greek mathematicians, who studied the problem of finding the shortest path joining two points in a sphere. Certainly, the notion of the shortest path joining two points is behind Euclid's 
postulates of plane geometry. Indeed, in the Euclidean plane the straight lines minimize the length of curves joining points in the plane, and in the hyperbolic upper-half plane the hyperbolic geodesics are straight lines and circles which minimize the length of curves with fixed endpoints. The definition of distance is variational in nature: it is the infimum of the lengths obtained by "varying" the curves joining two points.

Geodesics joining two points always exist in complete Riemannian manifolds by Hopf-Rinow's Theorem. With the aid of some basic features of the theory of Riemannian manifolds we can formulate rigourously a variational problem that leads to the solution of a differential equation whose solutions include the above minimizing geodesics.

\section{The Levi-Civita connection}

Recall that every Riemannian metric $g$ has a (unique) Levi-Civita connection

$$
\nabla: \mathcal{X}(M) \times \mathcal{X}(M) \longrightarrow \mathcal{X}(M),
$$

where $\mathcal{X}(M)$ is the set of smooth vector fields of $M$. The operator $\nabla$ acts as a differentiation of vector fields along curves in $M$ : the vector field $\nabla(X, Y)_{p}=\left(\nabla_{X} Y\right)_{p}$ evaluated at a point $p$ in $M$ is the same as the value of $\left(\nabla_{Z} Y\right)_{p}$ where $Z$ is a vector field in $M$ such that $Z(p)=X(p)$. In other words, the operator $\nabla$ acts as a derivation operator in $T M \times \mathcal{X}(M)$, this operator restricted to curves is called covariant differentiation.

The Levi-Civita connection satisfies the well known compatibility conditions with respect to the Riemannian metric: if $X, Y, Z$ are smooth vector fields in $M$, then

1. $X g(Y, Z)=g\left(\nabla_{X} Y, Z\right)+g\left(Y, \nabla_{X} Z\right)$,

2. $[X, Y]=\nabla_{X} Y-\nabla_{Y} X$,

where $[X, Y]$ is the Lie bracket of the vector fields $X$ and $Y$. The LeviCivita connection has an interpretation in terms of classical mechanics: it defines an acceleration of curves that is compatible with the metric, namely, the vector field

$$
\nabla_{\alpha^{\prime}(t)} \alpha^{\prime}(t)
$$

where $\alpha$ is a smooth curve in $M$. We say that a smooth vector field $X$ defined along a smooth curve $\alpha(t)$ is parallel if it satisfies the differential equation

$$
\nabla_{\alpha^{\prime}(t)} X(\alpha(t))=0 \text {. }
$$

We say that $X(\alpha(t))$ is the parallel transport of $X(0)$ along $\alpha$. It is easy to check that the parallel transport is an isometry, it preserves angles and norms of vectors. So we can view the parallel transport as a natural counterpart of the parallel transport of vectors in Euclidean space. 


\section{The differential equation of geodesics}

Given a smooth curve $\gamma:[0, T] \longrightarrow M$ with $\gamma(0)=x, \gamma(T)=y$, a smooth variation of $\gamma$ by curves is defined as a differentiable function $f$ : $(-\epsilon, \epsilon) \times[0,1] \longrightarrow M$ such that $f(0, t)=\gamma(t)$. We say that the variation $f$ has fixed endpoints $x$ and $y$ if $f((-\epsilon, \epsilon) \times\{0\})=x$, and $f((-\epsilon, \epsilon) \times\{1\})=y$. A variation of $\gamma$ can be thought as a local surface containing the curve $\gamma$. A natural way to think about the geodesic joining $x$ to $y$ is to define such a curve as minimum of the length of variations with endpoints $x, y$.

A common alternative approach in the literature about geodesics is to define them as minima of the so-called energy function of variations: the energy $E(c)$ of a smooth curve $c:[0, T] \longrightarrow M$ is given by $E(c)=\int_{0}^{T} g\left(c^{\prime}(t), c^{\prime}(t)\right) d t$. Observe that taking $[0, T]=[0,1]$, we get by the Schwartz inequality that $(L(c))^{2} \leq E(c)$, with equality if and only if the curve $c$ has constant speed. If we consider now the energy $E_{g}(f(s \times[0,1]))=E(s)$ of the curves of a variation $f(s, t)$, and take derivatives with respect to the parameter $s$ we obtain the well known first variation formula for the energy:

$$
\frac{\partial E}{\partial s}(0)=\int_{0}^{1} g\left(\nabla_{\gamma^{\prime}(t)} \gamma^{\prime}(t), \frac{\partial f}{\partial s}(0, s)\right) d t .
$$

Therefore, since the variational vector field $\frac{\partial f}{\partial s}(0, s)$ depends on the variation $f$ that is an arbitrary variation of $\gamma$, we conclude that a critical point $\gamma$ (a curve in fact) of the function $E(s)$ must satisfy the differential equation

$$
\nabla_{\gamma^{\prime}(t)} \gamma^{\prime}(t)=0
$$

This equation is a second order, linear, ordinary, differential equation, and hence, a solution $\gamma$ is uniquely determined by the initial conditions $\gamma(0)=$ $p, \gamma^{\prime}(0)=v$. Notice that the equation implies that the solutions have constant speed:

$$
g\left(\gamma^{\prime}(t), \gamma^{\prime}(t)\right)^{\prime}=2 g\left(\nabla_{\gamma^{\prime}(t)} \gamma^{\prime}(t), \gamma^{\prime}(t)\right)=0,
$$

according to the definition of the Levi-Civita connection (the derivative $g\left(\gamma^{\prime}(t), \gamma^{\prime}(t)\right)^{\prime}$ is a notation for the derivative of $g\left(\gamma^{\prime}(t), \gamma^{\prime}(t)\right)$ with respect to the vector field $\left.\gamma^{\prime}(t)\right)$. So the solutions $\gamma$ of the equation satisfy $L(\gamma)=$ $E(\gamma)^{\frac{1}{2}}$. Now, it is easy to check that the minima for $L(s)$ are just the minima for $E(s)$.

The solutions of the equation $\nabla_{\gamma^{\prime}(t)} \gamma^{\prime}(t)=0$ include of course those critical points of the energy function which might not be minimum points. However, a geodesic in the usual literature on Riemannian geometry is a solution of the above equation. The interpretation of the geodesics in terms of classical mechanics is that they are precisely the curves of the 
space with vanishing acceleration. So geodesics represent the free motion in a Riemannian manifold.

The first variation formula has many important consequences, we state next one of them because it will appear in many places in the survey.

Lemma 1.1. Let $(M, g)$ be a complete Riemannian manifold, and let $f$ : $(-\epsilon, \epsilon) \times[0, T] \longrightarrow M$ be a variation of a geodesic $\gamma(t)=f(0, t)$ that is parametrized by arc length. Then we have

$$
\frac{\partial E}{\partial s}(0)=g\left(\gamma^{\prime}(T), \frac{\partial f}{\partial s}(0, T)\right)-g\left(\gamma^{\prime}(0), \frac{\partial f}{\partial s}(0,0)\right) .
$$

The above formula tells us that when the variation of a geodesic $\gamma$ has no fixed endpoints, then the derivative of the length of the variation at $\gamma$ depends of the angles between $\gamma^{\prime}(t)$ and the variational vector field $\frac{\partial f}{\partial s}$ at $t=T$ and $t=0$. From this formula we can deduce that the distance from a point $p$ to a proper submanifold $S$ of $M$ is the length of a minimizing geodesic $\beta:[0,1] \longrightarrow M$ with $\beta(0)=p, \beta(1) \in S$, and $\beta^{\prime}(1)$ perpendicular to $T_{\beta(1)} S$.

The basic theory of Riemannian geometry shows that geodesics are indeed local minimizers: given a point $p$ in a complete Riemannian manifold $M$ there exists $\delta>0$ such that the open neighborhood $V_{\delta}(p)$ of radius $\delta$ centered at $p$ contains every geodesic segment $[x, y]$ joining any two points $x, y$ in $V_{\delta}(p)$, and this geodesic segment is the unique geodesic segment joining $x, y$. In particular, $[x, y]$ minimizes the length among all curves joining $x$ and $y$. Such a neighborhood is called normal or convex. This fact can be proved in many ways, one of them is calculating the second derivative of the length of variations (the second variation formula) and verifying that it is always positive for small variations with fixed endpoints (see for instance [31] for this approach). Another way of proving the local minimizing property of geodesics is using the theory of the exponential map that is the subject of the next subsection (see [29]).

\section{The exponential map, curvature and Ja- cobi fields}

The exponential map $\exp _{p}: T_{p} M \longrightarrow M$ at the point $p \in M$ is defined as $\exp _{p}(v)=\gamma_{v}(1)$, where $\gamma_{v}(t)$ is the geodesic whose initial conditions are $\gamma_{v}(0)=p, \gamma_{v}^{\prime}(0)=v$. Since geodesics have constant speed, we have that

$$
\exp _{p}(t v)=\gamma_{v}(t)
$$

for every unit vector $v \in T_{p} M$. This map is differentiable, and the uniqueness of geodesics in terms of their initial conditions grants that the $\exp _{p}$ is 
a local diffeomorphism (in fact the differential map $D_{0} e x p_{p}$ is the identity). Gauss (see [29] for instance) observed that geodesics starting at a point $p$ in a surface are perpendicular to small spheres centered at $p$. This fact holds in any dimension and is called Gauss Lemma, and with this property in hands we can define local polar coordinates in Riemannian manifolds with small metric spheres and geodesic rays. From the above observation it is not hard to show that geodesics are local minimizers as we mentioned in the previous subsection.

The exponential map is closely related with Jacobi fields and curvature.

Definition 1.1. Given three smooth, non-vanishing vector fields $X, Y, Z$ defined in an open neighborhood $U \subset M$, the curvature tensor $K(X, Y) Z$ is another vector field given by the formula

$$
R(X, Y) Z=\nabla_{X} \nabla_{Y} Z-\nabla_{Y} \nabla_{X} Z-\nabla_{[X, Y]} Z .
$$

The sectional curvature of the plane generated by $X(p), Y(p)$ is defined by

$$
K_{p}(X, Y)=\frac{g_{p}(R(X, Y) X, Y)}{|X \wedge Y|},
$$

where $|X \wedge Y|$ is the area of the parallelogram generated by $X(p), Y(p)$ with respect to the metric $g$.

It is not difficult to show that $K_{p}(X, Y)$ does not depend on the vector fields $X(p), Y(p)$ generating the same plane in the tangent space $T_{p}$. The function $K_{p}(X, Y)$ is just the Gaussian curvature in the case of surfaces, and if the surface is a submanifold of Euclidean space, it is of course the determinant of the second fundamental form. A classical argument due to Riemann shows that the sectional curvature $K_{p}(X, Y)$ is the curvature of the surface $\exp _{p}(t X(p)+s Y(p))$, for $t, s$ small, viewed as a submanifold of the ambient space $(M, g)$. So the curvature describes the shape of the local surfaces in $(M, g)$ given by the exponential of small open subsets of the tangent plane at every point.

Definition 1.2. A Jacobi vector field $J(t)$ defined along a geodesic $\gamma(t)$ is a vector function which is a solution of the differential equation

$$
J^{\prime \prime}(t)+R\left(\gamma^{\prime}(t), J(t)\right) \gamma^{\prime}(t)=0,
$$

where $J^{\prime}(t)=\nabla_{\gamma^{\prime}(t)} J(t)$ is the covariant derivative of the vector field $J(t)$ with respect to the vector $\gamma^{\prime}(t)$.

Let $\left.\gamma\right|_{(a, b)}$ be a geodesic, and let $f:(-\epsilon, \epsilon) \times(a, b) \longrightarrow M$ be a $C^{3}$ variation of $\gamma$ by geodesics, i.e., every curve $\alpha_{s_{0}}(t)=f\left(s_{0}, t\right), t \in(a, b)$, is a geodesic and $\alpha_{0}(t)=\gamma(t)$. The vector field

$$
J(t)=\left.\frac{\partial}{\partial s} f(s, t)\right|_{s=0}
$$


that is tangent to the variation along the geodesic $\gamma(t)$ is a Jacobi field, as proved by Jacobi. So, the qualitative theory of the Jacobi equation is closely related to the study of the infinitesimal behavior of geodesics.

As a solution of a second order differential equation, a Jacobi field is determined by the values of the vector field and its derivative at one point of the geodesic. The set of Jacobi fields of a geodesic is a finite dimensional vector space, and one of the most widely used tools to study the behavior of the norm of such vector fields is the comparison theory (Sturm-Liouville theory) for second order, ordinary differential equations. This is the reason why when the sectional curvatures have fixed sign a lot is known about the qualitative behavior of Jacobi fields. In Riemannian geometry, the comparison theory of Jacobi fields is called Rauch's comparison theory (see [79]).

The following result connects the exponential map with Jacobi fields.

Lemma 1.2. Let $J(t)$ be a Jacobi field defined in the geodesic $\gamma_{v}(t)$, such that $J(0)=0, J^{\prime}(0) \neq 0$. Then

$$
J(t)=D_{t v} \exp _{p}\left(t J^{\prime}(0)\right)
$$

The proof of the lemma is very simple: the differential map of $\exp _{p}$ applied to a vector $W \in T_{t v}\left(T_{p} M\right)$ is the derivative of a curve of the form $c_{t}(s)=\exp _{p}(t c(s))$, where $c(0)=v, c^{\prime}(0)=W$. But the function $f(t, s)=c_{t}(s)$ is a variation by geodesics, so its derivative with respect to $s$ is a Jacobi field.

A straightforward consequence of the lemma is that if the sectional curvatures are nonpositive, then the exponential map is always non-singular. From this fact we can deduce the well known Cartan-Hadamard Theorem:

Theorem 1.1. Let $(M, g)$ be a compact manifold with nonpositive curvature. Then the exponential map $\exp _{p}$ is a covering map for every $p \in M$.

The non-singularity of the exponential map at every point is a remarkable feature, it implies in particular that the universal covering of the manifold is $\mathbb{R}^{n}$. Riemannian manifolds with this property are called manifolds without conjugate points and manifolds with nonpositive curvature are examples of such manifolds.

Definition 1.3. A geodesic $\gamma$ of $(M, g)$ has no conjugate points if every Jacobi field defined in $\gamma$ has at most one zero.

An elementary application of Lemma 1.2 is the following.

Lemma 1.3. $(M, g)$ has no conjugate points if and only if every geodesic of $(M, g)$ has no conjugate points. 
The notion of geodesic without conjugate points goes back to variational calculus and Morse theory. We shall deal with manifolds without conjugate points in more detail in a forthcoming section. The most general result concerning the existence of geodesics is closely related to global existence theorems of ordinary differential equations and is known as the Hopf-Rinow Theorem. We state one of its many versions for the sake of completeness.

Theorem 1.2. Let $(M, g)$ be a $C^{\infty}$ Riemannian manifold. Then the following are equivalent:

1. $(M, g)$ is a complete metric space.

2. Given any two points $p, q$ in $M$ there exists a geodesic joining $p$ to $q$ such that $d(p, q)$ equals the length of this geodesic.

3. The exponential map $\exp _{p}$ is surjective for every $p \in M$.

4. The maximal interval of definition of any geodesic in $M$ (as a solution of an ordinary differential equation) is $\mathbb{R}$.

\section{The geodesic flow}

The interplay between variational ideas and ordinary differential equations proved to be very rich and convenient, allowing the introduction of many interesting objects related to geodesics. The equation of geodesics implies that the tangent vector of a geodesic must be parallel with respect to the connection, and in particular of constant norm, so it is often assumed that geodesics are always parametrized by arclength. We shall suppose throughout the exposition that geodesics have unit speed, we do not loose any information about the geometry of geodesics if we change their parametrizations.

The unit tangent bundle $T_{1} M$ of $(M, g)$ is the set of vectors whose norm is 1 in the norm induced by $g$. The previous observations about geodesics imply that we can define a flow in $T_{1} M$ whose orbits are natural lifts of geodesics. Let $\theta \in T_{1} M$ and let $(p, v)$ be the canonical coordinates of $\theta$, namely, $p \in M, v \in T_{p} M$.

Definition 1.4. The geodesic flow of $(M, g)$ is a one parameter family of diffeomorphisms, $\phi_{t}: T_{1} M \longrightarrow T_{1} M$ defined by

$$
\phi_{t}(p, v)=\left(\gamma_{(p, v)}(t), \gamma_{(p, v)}^{\prime}(t)\right)
$$

where $\gamma_{(p, v)}(t)$ is the geodesic whose initial conditions are $(p, v)$. 
This flow is $C^{\infty}$ if $(M, g)$ is $C^{\infty}$. The geodesic flow is complete if $(M, g)$ is complete, and it is clear that the geodesic flow sets up a frame to study geodesics that is much simpler in many respects than the manifold itself: the geodesics in $(M, g)$ do not form a flow, they cross each other as often as we wish. The map $\pi: T M \longrightarrow M$ given by $\pi(p, v)=p$ is called the canonical projection of the tangent bundle onto $M$. By the definition of the geodesic flow, the canonical projection of an orbit of the geodesic flow is a geodesic.

Let us denote by $\tilde{M}$ the universal covering of the manifold $M$, and by $\Pi: \tilde{M} \longrightarrow M$ the covering map. The metric $g$ induces a metric in $\tilde{M}$ called the pullback of $g$, denoted by $\tilde{g}$, and defined by $\tilde{g}(v, w)=g(D \Pi(v), D \Pi(w))$, where $D \Pi$ is the differential of the map $\Pi$. The covering transformations of $\tilde{M}$ become isometries in $(\tilde{M}, \tilde{g})$, and the covering map is a local isometry between $\tilde{M}$ and $M$. This implies that the geodesics of $(\tilde{M}, \tilde{g})$ are projected under the covering map onto geodesics of $(M, g)$, so the geodesics of $M$ can be canonically lifted to $\tilde{M}$. This simple idea is very important and useful in global Riemannian geometry, and we shall use it many times along the exposition.

\subsection{Sasaki metric, Jacobi fields and the differential of the geodesic flow}

The Riemannian metric $g$ defined in $M$ induces a natural Riemannian metric in $T_{1} M$ called the Sasaki metric. To construct the Sasaki metric we need some definitions.

Definition 1.5. Let $\pi: T_{1} M \longrightarrow M$ be the canonical projection, $\pi(p, v)=$ $p$. The vertical subspace at a point $\theta \in T_{1} M$ is the kernel of the map $D_{\theta} \pi$. We denote this subspace by $V_{\theta}$. The collection of vertical subspaces form a vector bundle called the vertical bundle of $T_{1} M$.

Definition 1.6. The horizontal subspace $\mathcal{H}_{\theta}$ at a point $(p, v) \in T_{1} M$ is the kernel of the connection map $\mathcal{K}: T T_{1} M \longrightarrow T M$ which is defined in the following way: let $Z \in T_{\theta} T_{1} M$, where $\theta=(p, v)$, and let $c(t)=(a(t), b(t))$, $t \in(-\epsilon, \epsilon)$, be a smooth curve in $T_{1} M$ such that $c(0)=\theta, c^{\prime}(0)=Z$; then

$$
\mathbb{K}(Z)=\nabla_{a^{\prime}(0)} b(0)=\nabla_{D \pi(Z)} b(0) .
$$

In the last definition, notice that $b(t)$ defines a vector field along the curve $a(t)$, and that if $b(t)$ is parallel along $a(t)$ then $c^{\prime}(0)$ is a horizontal vector of $T_{\theta} T_{1} M$. In particular, if $\gamma(t)$ is a unit speed geodesic with $\gamma(0)=p$, then the curve $\left(\gamma(t), \gamma^{\prime}(t)\right)$ is horizontal. Namely, the vector field tangent to the geodesic flow is always horizontal.

The vertical subspace has dimension $n-1$, and the horizontal subspace has dimension $n$ if $n$ is the dimension of $M$. We have that $T_{\theta} T_{1} M=\mathcal{H}_{\theta} \oplus V_{\theta}$, 
and that the horizontal subspace $\mathcal{H}_{(p, v)}$ is canonically isomorphic to $T_{p} M$ : since $D \pi$ is a submersion, given a vector $w \in T_{p} M$ there exists a unique vector $W$ in $\mathcal{H}_{(p, v)}$, called the horizontal lift of $w$, such that $D \pi(W)=w$. Clearly, the map $w \mapsto W$ is an isomorphism. The Sasaki metric $<,>$ in the tangent space of $T_{1} M$ is the metric which turns these subspaces into orthogonal subspaces,

$$
<X, Y>_{(p, v)}=g_{p}(D \pi(X), D \pi(Y))+g_{p}(\mathcal{K}(X), \mathcal{K}(Y)) .
$$

The map $D \pi$ is a Riemannian submersion with respect to this metric: the restriction of $D \pi$ to each horizontal subspace is an isometry.

Now, let $\gamma_{\theta}$ be a unit speed geodesic of $(M, g)$, where $\theta=(p, v) \in T_{1} M$ indicates the initial position and velocity of $\gamma_{\theta}$. Observe that $\gamma_{\theta}(t)=$ $\pi\left(\phi_{t}(\theta)\right)$.

Lemma 1.4. Given a vector $(V, W) \in T_{\theta} T_{1} M=\mathcal{H}_{\theta} \oplus V_{\theta}$, there exists a unique Jacobi field $J_{(V, W)}(t)$ defined in $\gamma_{\theta}$ whose initial conditions are $J_{(V, W)}(0)=V, J_{(V, W)}^{\prime}(0)=W$, such that

$$
D_{\theta} \phi_{t}(V, W)=\left(J_{(V, W)}(t), J_{(V, W)}^{\prime}(t)\right)
$$

in coordinates of $T_{\phi_{t}(\theta)} T_{1} M=\mathcal{H}_{\phi_{t}(\theta)} \oplus V_{\phi_{t}(\theta)}$.

This lemma is very important, it makes the link between the geometry of $(M, g)$ and the infinitesimal behavior of the dynamics of the geodesic flow. The lemma asserts essentially that the behavior of the differential of the geodesic flow is determined by the behavior of Jacobi fields, and hence, by the sectional curvatures of $(M, g)$.

One of the immediate consequences of Lemma 1.4 is a reduction of the study of the dynamics of $D \phi_{t}$ to the subbundle of $T T_{1} M$ that is perpendicular to the geodesic vector field. Let $N_{\theta}$ be the subspace of $T_{\theta} T_{1} M$ of vectors which are orthogonal to the geodesic flow with respect to the Sasaki metric, and let $\mathcal{N}$ be the subbundle whose elements are the subspaces $N_{\theta}$, $\theta \in T_{1} M$. Let $H_{\theta} \subset T_{\theta} T_{1} M$ be the intersection of the horizontal subspace of $\theta$ with the subspace $N_{\theta}$, and let $H$ be the subbundle of such subspaces.

Lemma 1.5. The subbundle $N$ is invariant by the geodesic flow.

Proof. This is a straightforward consequence of the basic theory of the Jacobi equation and Lemma 1.4, we sketch the proof for the sake of completeness. Let $\gamma$ be a unit speed geodesic in $(M, g)$, and let $J(t)$ be a Jacobi vector field defined in $\gamma$. If we differentiate twice the function $f(t)=g_{\gamma(t)}\left(J^{\prime}(t), \gamma^{\prime}(t)\right)$ we get that $f^{\prime}(t)=0$ for every $t \in \mathbb{R}$. Moreover, $g_{\gamma(t)}\left(J(t), \gamma^{\prime}(t)\right)^{\prime}=g_{\gamma(t)}\left(J^{\prime}(t), \gamma^{\prime}(t)\right)=g_{\gamma(0)}\left(J^{\prime}(0), \gamma^{\prime}(0)\right)$ for every $t \in \mathbb{R}$. Integrating the previous equation we obtain

$$
g_{\gamma(t)}\left(J(t), \gamma^{\prime}(t)\right)=g_{\gamma(0)}\left(J(0), \gamma^{\prime}(0)\right)+g_{\gamma(0)}\left(J^{\prime}(0), \gamma^{\prime}(0)\right) t .
$$


Therefore, if $J(0)=0$ and $g_{\gamma(0)}\left(J^{\prime}(0), \gamma^{\prime}(0)\right)=0$, the Jacobi field $J(t)$ stays perpendicular to $\gamma^{\prime}(t)$ for every $t \in \mathbb{R}$. The subspace of Jacobi fields generated by such fields has dimension $n-1$ and corresponds by Lemma 1.4 to vertical vectors. Let $W=\left(0, J^{\prime}(0)\right) \in V_{\theta}$, then Lemma 1.4 and the above formula imply that $D_{\theta} \phi_{t}(W)=\left(J(t), J^{\prime}(t)\right) \in N_{\phi_{t}(\theta)}$ for every $t \in \mathbb{R}$.

Now, let $J(t)$ be a Jacobi field such that $J^{\prime}(0)=0, g_{\gamma(0)}\left(J(0), \gamma^{\prime}(0)\right)=0$. Such fields generate a $n-1$ dimensional subspace of Jacobi fields, which corresponds to vectors in $H$ according to Lemma 1.4. The initial conditions of these Jacobi fields grant that $g_{\gamma(t)}\left(J(t), \gamma^{\prime}(t)\right)=0$ for every $t \in \mathbb{R}$, and again by Lemma 1.4 we obtain that $D_{\theta} \phi_{t}\left(H_{\theta}\right) \subset N_{\phi_{t}(\theta)}$ for every $t \in \mathbb{R}$.

Since $D_{\theta} \phi_{t}$ is a diffeomorphism for every $\theta \in T_{1} M, t \in \mathbb{R}$, and $H_{\theta}, V_{\theta}$ are linearly independent generators of $N_{\theta}$, we conclude that $D_{\theta} \phi_{t}\left(N_{\theta}\right)=N_{\phi_{t}(\theta)}$ as we wished to show.

The subspaces $N_{\theta}$ are very important in the symplectic theory of the geodesic flow, which is the subject of the next section.

\subsection{Transversal symplectic structure of the geodesic flow}

In this section we review some aspects of the theory of geodesic flows from the point of view of conservative dynamics. Our main references for the section are [3], [31]. The geodesic flow of $(M, g)$ is the Euler-Lagrange flow of the Lagrangian $L: T M \longrightarrow \mathbb{R}$ given by $L(x, v)=\frac{1}{2} g_{x}(v, v)$ in the energy level $E=1$, where the energy $E$ is just $E(x, v)=g_{x}(v, v)$. Recall that the Euler-Lagrange equation of a Lagrangian $L$ defined in $T M$ is

$$
\frac{d}{d t} L_{v}\left(x(t), x^{\prime}(t)\right)=L_{x}\left(x(t), x^{\prime}(t)\right)
$$

where $L_{v}, L_{x}$ are respectively notations for the gradients of $L$ with respect to the $v$-coordinates in $T_{x} M$, and the $x$-coordinates in $M$. In the case of the geodesic flow the Euler-Lagrange equation is simply $\nabla_{\gamma^{\prime}(t)} \gamma^{\prime}(t)=0$, where $\nabla$ is the Levi-Civita connection. As a particular case of mechanical Lagrangian, i.e., a Lagrangian of the form $L(x, v)=\frac{1}{2} g_{x}(v, v)-U(x)$, $U: M \longrightarrow \mathbb{R}$, the geodesic flow can be interpreted as the free motion in the manifold $(M, g)$ (in the case of the geodesic flow, $U(x)=0$ for every $x \in M)$.

The symplectic structure underlying the geodesic flow arises from the well known duality relating Lagrangian systems and Hamiltonian systems. Recall that a symplectic two-form $\Omega: \mathbb{R}^{n} \times \mathbb{R}^{n} \longrightarrow \mathbb{R}$ is closed, alternating, non-degenerate two-form. A symplectic form $\Omega: T M \longrightarrow \mathbb{R}$ is a function 
with the property that the restriction $\Omega_{p}: T_{p} M \longrightarrow \mathbb{R}$ to each tangent space $T_{p} M$ is a symplectic two-form. Of course, the dimension of $M$ has to be even if it admits a symplectic form.

The so-called Fenchel transform $H: T^{*} M \longrightarrow \mathbb{R}$ of a convex Lagrangian $L$ is given by:

$$
H(x, p)=\sup _{v \in T_{x} M}(p(v)-L(x, v)),
$$

where $T^{*} M$ is the cotangent bundle of $M$ and $(x, p)$ are canonical local coordinates for $T^{*} M$. The function $H$ is called the Hamiltonian associated to $L$. The expression of $H$ in the case of the geodesic flow is very simple. Indeed, let $x=\left(x_{1}, x_{2}, \ldots x_{n}\right)$ be local coordinates for $M$, and let $v \in T_{x} M$ be given by $v=\left(v_{1}, v_{2}, . ., v_{n}\right)$ in the coordinates $\frac{\delta}{\delta x_{1}}, \frac{\delta}{\delta x_{2}}, \ldots, \frac{\delta}{\delta x_{n}}$ of $T_{x} M$. Let $\sigma: T M \longrightarrow T^{*} M$ be the dual map induced by the metric $g$, given in local coordinates by

$$
\sigma_{p}(v) \cdot w=g_{p}(v, w)=\sum_{i, j=1}^{n} g_{p}^{i j} v_{i} w_{j},
$$

where recall that $\sigma$ is a 1 -form, $p \in M, w \in T_{p} M$, and $g^{i j}$ are the coefficients of the metric $g$ in the coordinate basis.

Then we get in the case of the geodesic flow that the Hamiltonian $H$ is just

$$
H(x, p)=\frac{1}{2} g_{x}\left(\sigma^{-1}(p), \sigma^{-1}(p)\right) .
$$

Once we have a smooth function $H: T^{*} M \longrightarrow \mathbb{R}$ and a symplectic two form $\Omega: T T^{*} M \longrightarrow \mathbb{R}$, we define the Hamiltonian vector field $X_{H}$ by the differential equation

$$
D_{(x, p)} H(Y)=\Omega\left(X_{H}(x, p), Y\right),
$$

where $Y \in T_{(x, p)} T^{*} M$ and $D_{(x, p)} H$ is the differential of $H$. The vector field $X_{H}$ is often called the symplectic gradient of $H$ with respect to $\Omega$. If we write the differential equation in local coordinates $(x, p)$ of $T^{*} M$ we get the Hamilton equations

$$
\begin{gathered}
x^{\prime}(t)=H_{p}(x(t), p(t)), \\
p^{\prime}(t)=-H_{x}(x(t), p(t)),
\end{gathered}
$$

where $H_{p}, H_{x}$ are respectively the gradients with respect to the coordinates $p, x$.

There are natural differential forms associated to the cotangent bundle $T^{*} M$. The canonical (Liouville) 1 -form $\omega: T M \longrightarrow \mathbb{R}$ is given by

$$
\omega_{(x, p)}(W)=p\left(D_{x} \pi^{*}(W)\right)
$$


where $W \in T_{(x, p)} T^{*} M$ and $\pi^{*}: T^{*} M \longrightarrow M$ is the canonical projection. In local coordinates, the canonical 1 -form is given by

$$
\omega_{(x, p)}=p \cdot d x=\sum_{i=1}^{n} p_{i} d x_{i} .
$$

The canonical symplectic form associated to $H$ is defined by

$$
\Omega=d \omega=d p \wedge d x=\sum_{i=1}^{n} d p_{i} \wedge d x_{i} .
$$

The geodesic flow in $T^{*} M$ is defined by the solutions of the symplectic gradient of $H(x, p)=\frac{1}{2} g_{p}\left(\sigma^{-1}(p), \sigma^{-1}(p)\right)$ with respect to the canonical symplectic two-form $\Omega$.

It is not difficult to check that the Euler-Lagrange flow of a convex Lagrangian and the Hamiltonian flow associated to its Fenchel transform are conjugate by the Legendre transform $\mathbb{L}: T M \longrightarrow T^{*} M$ :

$$
\mathbb{L}(x, v)=\left(x, L_{v}(x, v)\right),
$$

where $L_{v}$ is the gradient of $L$ with respect to the canonical coordinates of the tangent space $T_{x} M$ (the vertical coordinates in fact). In the case of geodesic flows, the Legendre transform is just the dual map $\sigma$.

A straightforward calculation in local coordinates gives that the pullbacks $\mathbb{L}^{*}(\omega): T T M \longrightarrow \mathbb{R}, \mathbb{L}^{*}(\Omega): T T M \longrightarrow \mathbb{R}$ of the forms $\omega, \Omega$, by the Legendre transform, are the forms

$$
\begin{gathered}
\mathbb{L}^{*}(\omega)(x, v)(W)=<W, X(x, v)>, \\
\mathbb{L}^{*}(\Omega)(x, v)(W, Z)=g_{x}\left(P_{(x, v)}^{\mathcal{H}}(W), P_{(x, v)}^{V}(Z)\right)-g_{x}\left(P_{(x, v)}^{\mathcal{H}}(Z), P_{(x, v)}^{V}(W)\right),
\end{gathered}
$$

where $\langle W, X\rangle$ is the Sasaki metric, $X(x, v)$ is the geodesic vector field, $P_{(x, v)}^{\mathcal{H}}: T_{(x, v)} T M \longrightarrow \mathcal{H}_{(x, v)}$ is the orthogonal projection in the horizontal space, and $P_{(x, v)}^{V}: T_{(x, v)} T M \longrightarrow V_{(x, v)}$ is the orthogonal projection in the vertical subspace.

Since we shall work mainly in the Lagrangian framework of the geodesic flow, we shall use the same notations $\omega, \Omega$ for the pullbacks of the canonical forms. Their restrictions to $T T_{1} M$ will be denoted by $\omega, \Omega$ too. Remark that the restriction of $\Omega$ to $T T_{1} M$ is not a symplectic form, because the dimension of $T_{1} M$ is odd. However, the restriction $\Omega^{\perp}: \mathcal{N} \longrightarrow \mathbb{R}$ to the subbundle $\mathcal{N}$ defined in the previous section gives a symplectic form. Its expression is

$$
\Omega^{\perp}(x, v)(W, Z)=g_{x}\left(P_{(x, v)}^{H}(W), P_{(x, v)}^{V}(Z)\right)-g_{x}\left(P_{(x, v)}^{H}(Z), P_{(x, v)}^{V}(W)\right),
$$


where $P_{(x, v)}^{H}: T_{(x, v)} T M \longrightarrow H_{(x, v)}$ is the orthogonal projection in the subspace $H_{(x, v)}=\mathcal{H}_{(x, v)} \cap N_{(x, v)}$. A fundamental fact of the theory of geodesic flows is that these forms are invariant by the differential of the geodesic flow. In a standard reference of mathematical physics, the invariance of the canonical forms is shown using the Hamiltonian formalism. We shall use the Lagrangian point of view just to apply the results of the previous sections.

Lemma 1.6. The canonical forms $\omega, \Omega^{\perp}$ are invariant by the geodesic flow in $T_{1} M$. Namely, $\phi_{t}^{*}(\omega)=\omega, \phi_{t}^{*}\left(\Omega^{\perp}\right)=\Omega^{\perp}$ for every $t \in \mathbb{R}$, where $\phi_{t}^{*} \Theta\left(w_{1}, w_{2}, . ., w_{k}\right)=\Theta\left(D \phi_{t}\left(w_{1}\right), . ., D \phi_{t}\left(w_{k}\right)\right)$ for every $k$-form $\Theta$.

Proof. According to Lemma 1.4, given a vector $(W, Z) \in T_{\theta} T_{1} M=\mathcal{H}_{\theta} \oplus$ $V_{\theta}$, there exists a unique Jacobi field $J_{(W, Z)}(t)$ defined in $\gamma_{\theta}$ whose initial conditions are $J_{(W, Z)}(0)=W, J_{(W, Z)}^{\prime}(0)=Z$, such that

$$
D_{\theta} \phi_{t}(W, Z)=\left(J_{(W, Z)}(t), J_{(W, Z)}^{\prime}(t)\right)
$$

in coordinates of $T_{\phi_{t}(\theta)} T_{1} M=\mathcal{H}_{\phi_{t}(\theta)} \oplus V_{\phi_{t}(\theta)}$. Let $X(\theta)$ be the geodesic vector field. Writing $D_{\theta} \phi_{t}(W, Z)$ in the coordinates $X\left(\phi_{t}(\theta)\right) \oplus N_{\phi_{t}(\theta)}$ we get

$$
\begin{aligned}
D_{\theta} \phi_{t}(W, Z)= & g_{\gamma(t)}\left(J_{(W, Z)}(t), \gamma^{\prime}(t)\right) X\left(\phi_{t}(\theta)\right) \\
& +P_{N}\left(J_{(W, Z)}(t), J_{(W, Z)}^{\prime}(t)\right) N_{\phi_{t}(\theta)} .
\end{aligned}
$$

So the canonical one-form $\omega$ evaluated in $D_{\theta} \phi_{t}(W, Z)$ gives

$$
\omega\left(D_{\theta} \phi_{t}(W, Z)\right)=g_{\gamma(t)}\left(J_{(W, Z)}(t), \gamma^{\prime}(t)\right) .
$$

By Lemma 1.5 we have that

$$
\begin{aligned}
g_{\gamma(t)}\left(J_{(W, Z)}(t), \gamma^{\prime}(t)\right) & =g_{\gamma(0)}\left(J_{(W, Z)}(0), \gamma^{\prime}(0)\right)+g_{\gamma(0)}\left(J_{(W, Z)}^{\prime}(0), \gamma^{\prime}(0)\right) t \\
& =\omega(W, Z)+g_{\gamma(0)}\left(J_{(W, Z)}^{\prime}(0), \gamma^{\prime}(0)\right) t .
\end{aligned}
$$

Therefore, if we assume that $g_{\gamma(0)}\left(J_{(W, Z)}^{\prime}(0), \gamma^{\prime}(0)\right)=0$ we have that $\phi_{t}^{*} \omega(W, Z)=\omega(W, Z)$ as we wish to show. The point is that the Jacobi fields with this condition together with parallel Jacobi fields generate all Jacobi fields in $\gamma$. And it is clear that the form $\omega$ is $\phi_{t}$-invariant along parallel Jacobi fields. This shows that $\omega$ is $\phi_{t}$-invariant.

Regarding the symplectic two-form $\Omega^{\perp}$, we get by elementary calculus of differential forms that if $f=\phi_{t}$,

$$
f^{*}(\Omega)=f^{*}(d \omega)=d\left(f^{*}(\omega)\right)=d(\omega)=\Omega,
$$

so the symplectic form $\Omega$ in $T M$ is $\phi_{t}$-invariant. Since the subbundle $N$ is $D \phi_{t}$-invariant too, we get that $\Omega^{\perp}$ is invariant by the geodesic flow. 
Another nice way of proving that $\Omega^{\perp}$ is $\phi_{t}$-invariant is using elementary properties of the Jacobi equation. In fact, we have,

$$
\begin{aligned}
\Omega^{\perp}\left(D_{\theta} \phi_{t}\left(W_{1}, Z_{1}\right), D_{\theta} \phi_{t}\left(W_{2}, Z_{2}\right)\right)= & g_{\gamma(t)}\left(J_{\left(W_{1}, Z_{1}\right)}(t), J_{\left(W_{2}, Z_{2}\right)}^{\prime}(t)\right) \\
& -g_{\gamma(t)}\left(J_{\left(W_{1}, Z_{1}\right)}^{\prime}(t), J_{\left(W_{2}, Z_{2}\right)}(t)\right) .
\end{aligned}
$$

This quantity is nothing but the Wronskian of the solutions $J_{\left(W_{1}, Z_{1}\right)}(t)$, $J_{\left(W_{2}, Z_{2}\right)}(t)$ that is constant for the Jacobi equation. This implies that $\phi_{t}^{*}\left(\Omega^{\perp}\right)=\Omega^{\perp}$.

Corollary 1.1. The geodesic flow preserves the measure $\mu=\left(\Omega^{\perp}\right)^{n-1} \wedge \omega$, the Liouville measure.

The Liouville measure is in fact the Lebesgue measure of $T_{1} M$. So this corollary has many strong consequences for the dynamics of the flow and is the starting point of the smooth ergodic theory of the geodesic flow. We shall be more concerned in the survey with topological dynamics than ergodic theory, so we won't go very deep into this subject in the present text. However, the symplectic structure of the geodesic flow and little of ergodic theory will be used in forthcoming sections to illustrate some important applications of $C^{1}$ methods to study its dynamics. We would like to remind one of the main theorems of ergodic theory, due to Birkhoff [63]. We state the theorem for the case of flows.

Theorem 1.3. Let $\psi_{t}: N \longrightarrow N$ be a smooth flow, where $N$ is a complete smooth manifold. Suppose that $N$ supports a probability measure $\mu$ that is invariant by $\psi_{t}$, i.e., for every measurable set $A \subset N, \mu\left(\psi_{t}(A)\right)=\mu(A)$ for every $t$ in the set of parameters. Let $f: N \longrightarrow \mathbb{R}$ be a measurable, integrable function. Then

1. The function

$$
\bar{f}(x)=\lim _{t \rightarrow+-\infty} \frac{1}{t} \int_{0}^{t} f\left(\psi_{s}(x)\right) d x
$$

is a well defined limit in a total measure subset of $N$.

2. $\bar{f}$ is integrable and $\psi_{t}$-invariant: $\bar{f}\left(\psi_{t}(x)\right)=\bar{f}(x)$ for almost every $x$ in $N$.

3. The integral of $\bar{f}$ satisfies

$$
\int_{N} \bar{f}(x) d \mu=\int_{N} f(x) d \mu .
$$

\section{The universal covering}

We devote the end of the chapter to give a crash course about the theory of covering spaces. Given two topological spaces $X, Y$ and a continuous 
map $\Pi: X \longrightarrow Y$, we say that $\Pi$ is a covering map if for every $p \in X$ there exists an open neighborhood $U_{p} \subset X$ containing $p$ such that the restriction of $\Pi$ to $U_{p}$ is a homeomorphism. Given a topological space $Y$, the collection $C_{Y}$ of topological spaces $X$ such that there exists a covering map $\Pi_{X}: X \longrightarrow Y$ admits a natural partial order: $X_{1} \leq X_{2}$ if there exists a covering map $\sigma: X_{2} \longrightarrow X_{1}$. By Zorn's Lemma, there is a maximal element $\tilde{Y}$ in $C_{Y}$ which is called the universal covering of $Y$. The universal covering is unique up to homeomorphisms.

The key property of covering spaces is the so-called lifting property of maps. Namely,

Lemma 1.7. Let $\Pi: X \longrightarrow Y$ be a covering map between two topological spaces $X, Y$, and let $f: Z \longrightarrow Y$ be a continuous map from a topological space $Z$ to $Y$. Let $z \in Z$ and $q \in \Pi^{-1}(f(z))$. Then there exists a unique continuous map $\tilde{f}: Z \longrightarrow X$ such that $\Pi \circ \tilde{f}=f$ and $\tilde{f}(z)=q$.

The proof of Lemma 1.7 is based on the fact that the covering map $\Pi$ is a local homeomorphism: we can construct local lifts of $f$ using local inverses of $\Pi$. Then we can patch such local lifts and get a lift $\tilde{f}$ of $f$. We leave the details to the reader. The map $\tilde{f}$ is called a lift of $f$, and in general there might be many lifts of a single map.

\subsection{Homotopy classes}

One of the most relevant aspects of the theory of covering spaces is its connection with homotopy theory, isometric actions and uniformization theory. We recall next the basic notions of the homotopy theory of curves. The above theory is suited for path connected spaces, so from now on we shall assume that our topological spaces are just smooth, connected manifolds.

Given a smooth manifold $M$, and two continuous curves $c:[0,1] \longrightarrow M$, $w:[0,1] \longrightarrow M$, a homotopy from $c$ to $w$ is a continuous map $F:[0,1] \times$ $[0,1] \longrightarrow M$ such that

1. $F(0, t)=c(t)$ for every $t \in[0,1]$,

2. $F(1, t)=w(t)$ for every $t \in[0,1]$.

When $c(0)=c(1)$ we say that $c$ is a closed curve or a loop. If $c(1)=w(0)$ we define the composition $c \circ w:[0,1] \longrightarrow M$ as follows:

1. $c \circ w(t)=c(2 t)$ for every $t \in\left[0, \frac{1}{2}\right]$,

2. $c \circ w(t)=w(2 t-1)$ for every $t \in\left[\frac{1}{2}, 1\right]$. 
Two closed curves $c_{1}, c_{2}$ are called homotopic or freely homotopic if there exists an homotopy from $c_{1}$ to $c_{2}$. The homotopy relation between closed curves is an equivalence relation, and the set of equivalence classes is called the free homotopy group.

Given $p \in M$, the collection $\Omega_{p}$ of loops $c$ with $c(0)=c(1)=p$ is called the space of loops with base point $p$. The composition of loops in $\Omega_{p}$ is well defined and gives another element of $\Omega_{p}$. A homotopy $F$ from $c \in \Omega_{p}$ to $w \Omega_{p}$ with base point $p$ is a homotopy from $c$ to $w$ such that $F(s, 0)=F(s, 1)=p$ for every $s \in[0,1]$. The homotopy $F$ with base point $p$ can be viewed as a path of curves in $\Omega_{p}$ from $c$ to $w$. The curves $c, w$ in $\Omega_{p}$ are called homotopic if there exists a homotopy from $c$ to $w$ with base point $p$. A loop in $\Omega_{p}$ is homotopically trivial if it is homotopic to the constant loop $p$. $M$ is said to be simply connected if every loop in $\Omega_{p}$ is homotopically trivial for some $p \in M$. With the help of the lifting property (Lemma 1.7 we can show that the universal covering is simply connected.

It is easy to check that the property of being homotopic in $\Omega_{p}$ defines an equivalence relation in $\Omega_{p}$. Moreover, the composition of loops induces a natural operation in the set of pairs of equivalence classes which is a group operation. So the set of equivalence classes in $\Omega_{p}$ endowed with this group operation is called the fundamental group $\pi_{1}(M, p)$ with base point $p$ of $M$. It is easy to show that $\pi_{1}(M, p)$ and $\pi_{1}(M, q)$ are isomorphic (in fact, they are conjugate) for every $p, q \in M$. So we shall denote by $\pi_{1}(M)$ the conjugacy class of the base point fundamental groups of $M$, and we shall refer to it as the fundamental group of $M$.

One of the fundamental links between homotopy groups and geometry is the following famous theorem due of Birkhoff.

Theorem 1.4. Let $(M, g)$ be a $C^{\infty}$ complete (as a metric space) Riemannian manifold. Then in each nontrivial homotopy class there exists a closed geodesic whose length is minimal in the class.

\subsection{Covering transformations and fundamental group}

A covering transformation $F: \tilde{M} \longrightarrow \tilde{M}$ is a homeomorphism satisfying $\Pi \circ F=\Pi$. The basic theory of covering spaces shows that covering transformations are closely related with the elements of the fundamental group, we explain briefly this connection for the sake of completeness.

Given an element $[\alpha] \in \pi_{1}(M, p)$, where $\alpha:[0,1] \longrightarrow M$ is a loop with base point $p$, and a lift $\tilde{\alpha} \in \tilde{M}$ with $\tilde{\alpha}(0)=\tilde{p} \in \Pi^{-1}(p)$, there is a natural homeomorphism $T_{[\alpha]}: \tilde{M} \longrightarrow \tilde{M}$ associated to $\alpha$ which is defined as follows. Let $\tilde{q} \in \tilde{M}$, to get $T_{[\alpha]}(\tilde{q})$ we consider a continuous curve $\tilde{\beta}:[0,1] \longrightarrow \tilde{M}$ 
joining $\tilde{q}$ to $\tilde{p}$. This curve projects onto a curve $\Pi(\tilde{\beta})=\beta:[0,1] \longrightarrow M$ joining $q$ to $p$. The curve $\Gamma_{\beta}=\beta^{-1} \circ \alpha \circ \beta$ defines a loop $\Gamma_{\beta}:[0,1] \longrightarrow M$ based on $q$, where $\beta^{-1}$ is just a notation for the curve $\beta^{-1}(t)=\beta(1-t)$. By Lemma 1.7 we can lift this curve to a curve $\tilde{\Gamma}_{\beta} \subset \tilde{M}$ starting from $\tilde{q}=\tilde{\Gamma}_{\beta}(0)$, in a way that $\tilde{\alpha} \subset \tilde{\Gamma}_{\beta}$. The point $T_{[\alpha]}(\tilde{q})$ is given by

$$
T_{[\alpha]}(\tilde{q})=\tilde{\Gamma}_{\beta}(1) .
$$

It is easy to show that this point does not depend on the choice of $\beta$, so it is well defined. Moreover, $T_{[\alpha]}$ is a homeomorphism of $\tilde{M}$ without fixed points, and if we replace $\tilde{\alpha}$ by another lift of $\alpha$, we get a homeomorphism $S: \tilde{M} \longrightarrow \tilde{M}$ that is conjugate to $T_{[\alpha]}$. So in fact $T_{[\alpha]}$ also depends on $\tilde{\alpha}$, but we won't make this explicit in the notation. The maps $T_{[\alpha]}$ are covering transformations, and they provide a natural representation of $\pi_{1}(M, p)$ in the group of homeomorphisms of $\tilde{M}$. The maps $T_{[\alpha]}$ can be viewed as geometric realizations of the elements of the fundamental group.

The main basic feature of the covering transformations is the following result.

Lemma 1.8. Let $M$ be a smooth manifold. Given $p \in M$, we have that $M$ is diffeomorphic to the quotient space given by the orbit space of the action of $\pi_{1}(M, p)$ on $\tilde{M}$.

\subsection{Covering transformations as isometries}

Given a Riemannian metric $g$ defined in $M$, the covering map allow us to give a natural metric $\tilde{g}$ in $\tilde{M}$, the pullback of $g$ by the covering map. It is defined by

$$
\tilde{g}_{\tilde{x}}(V, W)=g_{x}\left(D_{\tilde{x}} \Pi(V), D_{\tilde{x}} \Pi(W)\right)
$$

where $V, W \in T_{\tilde{x}} \tilde{M}$, and $\Pi(\tilde{x})=x$. The manifold $(\tilde{M}, \tilde{g})$ is locally isometric to $(M, g)$ and the geodesics of $(\tilde{M}, \tilde{g})$ are lifts of the geodesics in $(M, g)$.

The fundamental group $\pi_{1}(M, p)$ acts as a group of isometries in $(\tilde{M}, \tilde{g})$ by the very definition of the covering transformations associated to the fundamental group. Combining this observation and Lemma 1.8 we have that every connected Riemannian manifold can be obtained as the quotient of a simply connected Riemannian manifold by the action of a discrete subgroup of isometries. This simple but relevant fact will be very important for the sequel, it leads naturally to many considerations and problems in uniformization theory. 


\section{Chapter 2}

\section{Dynamical systems and stability}

The purpose of the chapter is to develop some basic notions and tools of the theory of dynamical systems related to the study of global stability of systems. A dynamical system is a continuous action $\phi: \Gamma \times M \longrightarrow M$ of a group $\Gamma$ on a topological space $M$, where $\Gamma$ is taken to be either $\mathbb{R}$ or $\mathbb{Z}$. If $M$ is a $C^{\infty}$ manifold and the action is $C^{k}$, we say that $\phi$ is a $C^{k}$ dynamical system.

Smooth flows in complete manifolds $\phi_{t}: M \longrightarrow M$ whose orbits are solutions of a first order ordinary differential equation are well known examples of smooth dynamical systems or $\mathbb{R}$-actions. The geodesic flow is just one of such examples. As examples of $\mathbb{Z}$-actions or discrete actions we might mention the action of smooth endomorphisms of manifolds and its iterates.

Many relevant physical models are given in terms of dynamical systems, like iteration processes and conservative dynamical systems coming from classical mechanics. The study of dynamical systems as models leads naturally to look at the evolution of initial conditions of the space under the action of the system. Such evolution gives rise to asymptotic configurations of the system, whose study is one of the main subjects of the general theory of dynamical systems. We shall discuss in the chapter some of the main results of the asymptotic theory of systems, notably in the case of systems enjoying some amount of hyperbolicity.

\section{Limit set and non-wandering set}

The complexity of the dynamics of a system is concentrated in the so-called limit set of the system. 
Definition 2.1. Given a continuous dynamical system $\phi: \Gamma \times M \longrightarrow M$ acting on a complete metric space $M$, the limit set of a point $p \in M$ is the set $L(p) \subset M$ given by the following property: for every $q \in L(p)$, there exists a sequence $\Gamma_{k}$ of elements in $\Gamma$ such that

$$
\lim _{k \rightarrow+\infty} d\left(q, \phi\left(\Gamma_{k}, p\right)\right)=0 .
$$

The $\alpha$-limit set $\alpha(p)$ of $p$ is the subset of $L(p)$ given by the limits of sequences $\phi\left(\Gamma_{k}, p\right)$ with $\Gamma_{k} \rightarrow+\infty$. The $\omega$-limit set $\omega(p)$ of $p$ is given by the limits of sequences $\phi\left(\Gamma_{k}, p\right)$ with $\Gamma_{k} \rightarrow-\infty$. The limit set $L(\phi)$ of a dynamical system $\phi$ is the union of the limit sets of the points in the space.

Definition 2.2. Given a continuous dynamical system $\phi: \Gamma \times M \longrightarrow M$ acting on a complete metric space $M$, the set $N W(\phi)$ of non-wandering points is defined as follows: $p \in N W(\phi)$ if and only if for every open neighborhood $V$ of $p$ there exists $n \in \Gamma n \geq 1$, such that $V \cap \phi(n, V) \neq \emptyset$, where $\phi(n, V)=\{\phi(n, x), x \in V\}$.

Every point in the limit set of a system is non-wandering, and the set of non-wandering points is a closed, invariant set for the system. If $M$ is compact, the set of non-wandering points is nonempty. A natural question in the theory of dynamical systems is to know whether the limit set coincides with the non-wandering set. It happens to be that the answer to this question is related in many cases with the stability and the hyperbolicity of the system. We shall come back to this point later in the chapter.

Conservative dynamical systems enjoy many special properties which are not found in general systems. One of the main basic results of conservative systems, whose proof is a straightforward exercise, is the following.

Lemma 2.1. Let $(M, \mu)$ be a probability space endowed with a probability measure $\mu$. Let $f: M \longrightarrow M$ be a smooth homeomorphism which preserves $\mu$ : for every subset $A \subset M$ we have that $\mu(f(A))=\mu(A)$. Then every point in the support of $\mu$ is non-wandering.

In fact, Lemma 2.1 can be improved.

Definition 2.3. Let $\phi: \Gamma \times M \longrightarrow M$ be a continuous dynamical system acting on a complete metric space $M$. A point $p \in M$ is called positively recurrent if there exists a sequence $\Gamma_{n} \in \Gamma$ with $\Gamma_{n} \rightarrow+\infty$, such that $\lim _{n \rightarrow+\infty} \phi\left(\Gamma_{n}, p\right)=p$. A point $q$ is called negatively recurrent if the same above assertion holds with $\Gamma_{n} \rightarrow-\infty$.

It is clear that the set of recurrent points is a special subset of the set of non-wandering points. The following result can be easily deduced from Birkhoff's theorem (Theorem 1.3). 
Lemma 2.2. Let $(M, \mu)$ be a probability space, endowed with a probability measure $\mu$. Let $f: M \longrightarrow M$ be a smooth homeomorphism which preserves $\mu$. Then the set of recurrent points of $f$ has total measure.

Lemmas 2.1 and 2.2 have analogous versions for nonsingular conservative flows. In particular, such lemmas hold for geodesic flows of compact Riemannian manifolds.

\section{Hyperbolic sets and Anosov dynamics}

We introduce in this section the notion of hyperbolic set of a dynamical system. We define first hyperbolic sets for diffeomorphism and then for flows.

Definition 2.4. Let $f: M \longrightarrow M$ be a $C^{\infty}$ diffeomorphism acting in a $C^{\infty}$ Riemannian manifold $M$. A closed invariant subset $A \subset M$ for $f$ is called hyperbolic if there exist constants, $C>0, \lambda \in(0,1)$, and a direct sum decomposition by invariant subspaces $T_{p} M=E^{s}(p) \oplus E^{u}(p)$ for every $p \in A$, such that

$$
\begin{aligned}
& \text { 1. }\left\|D f^{n}(W)\right\| \leq C \lambda^{n}\|W\| \text { for every } W \in E^{s}(p) \text { and } n \geq 0 \text {, } \\
& \text { 2. }\left\|D f^{n}(W)\right\| \leq C \lambda^{-n}\|W\| \text { for every } W \in E^{u}(p) \text { and } n \leq 0 .
\end{aligned}
$$

Definition 2.5. Let $\psi_{t}: M \longrightarrow M$ be a $C^{\infty}$ flow acting without singularities on a complete manifold $M$. A closed invariant subset $A \subset M$ is called hyperbolic for the flow if there exist constants, $C>0, \lambda \in(0,1)$, and a direct sum decomposition by invariant subspaces $T_{p} M=E^{s}(p) \oplus E^{u}(p) \oplus X(p)$ for every $p \in A$, where $X(p)$ is the subspace tangent to the orbits of the flow $\psi_{t}$, such that

$$
\begin{aligned}
& \text { 1. }\left\|D \psi_{t}(W)\right\| \leq C \lambda^{t}\|W\| \text { for every } W \in E^{s}(p) \text { and } t \geq 0 \text {, } \\
& \text { 2. }\left\|D \psi_{t}(W)\right\| \leq C \lambda^{-t}\|W\| \text { for every } W \in E^{u}(p) \text { and } t \leq 0 \text {. }
\end{aligned}
$$

The subspace $E^{s}(p)$ is usually called the stable subspace, and the subspace $E^{u}(p)$ is called the unstable subspace. When the set of nonwandering points of a diffeomorphism $f$ is a hyperbolic set, then the diffeomorphism is called Axiom A. If the manifold $M$ is a hyperbolic set then the dynamical system is called an Anosov system, in the honor of D. Anosov who developed a fairly complete theory of such systems in the 1960's after the works of E. Hopf and G. Hedlund about geodesic flows of compact surfaces of negative curvature. We shall come back to this issue in Chapter 3.

The theory developed by D. Anosov included an accurate analysis of the topological dynamics of the systems, which led to the proof of the structural 
stability; and the study of statistical or ergodic features of the dynamics. Since the emphasis of this survey is more topological than ergodic, we shall discuss for the sake of completeness the main ideas involved in the proof of the structural stability of Anosov systems.

The first fundamental property of compact hyperbolic sets is the existence of invariant submanifolds which are tangent to the invariant subspaces $E^{s}(p), E^{u}(p)$ at every $p \in A$. This result is often called the Invariant manifold Theorem, and generalizes the existence of invariant submanifolds of hyperbolic periodic orbits of systems. We follow [48].

Theorem 2.1. Let $\phi: \Gamma \times M \longrightarrow M$ be a $C^{1}$ dynamical system given by either a diffeomorphism $(\Gamma=\mathbb{Z})$ or a smooth flow without singularities $(\Gamma=\mathbb{R})$, acting in a $C^{\infty}$ Riemannian manifold $M$, and let $A \subset M$ be a compact, invariant hyperbolic set for $\phi$. Then for each $p \in A$ there is a pair of embedded, $C^{1}$ disks $W_{\text {loc }}^{s}(p), W_{\text {loc }}^{u}(p)$, called the local stable and the local unstable submanifolds of $p$ respectively, such that

1. The tangent space of $W_{l o c}^{s}(p)$ at $p$ is $E^{s}(p)$, and the tangent space of $W_{l o c}^{u}(p)$ at $p$ is $E^{u}(p)$.

2. $\left.\phi\left(W_{\text {loc }}^{s}(p), t\right)\right) \subset W_{\text {loc }}^{s}(\phi(p, t)), \phi\left(W_{\text {loc }}^{u}(p),-t\right) \subset W_{\text {loc }}^{u}(\phi(p,-t))$, for every $t \geq 0$,

3. For every $\delta>0$ there exists $C(\delta)$ such that

$$
d(\phi(p, t), \phi(q, n))<C(\delta)(\lambda+\delta)^{t} d(p, q),
$$

for every $q \in W_{\text {loc }}^{s}(p)$ and $t \geq 0$;

$$
d(\phi(p,-t), \phi(q,-t))<C(\delta)(\lambda-\delta)^{-t} d(p, q),
$$

for every $q \in W_{l o c}^{u}(p)$ and $t \geq 0$.

4. There exists $\beta>0$ and a family of neighborhoods $U_{p}$ which contain the ball of radius $\beta$ around $p$ such that

$$
\begin{gathered}
W_{l o c}^{s}(p)=\left\{q \in M, \phi(q, t) \in U_{\phi(p, t)} \forall t \geq 0\right\}, \\
W_{l o c}^{u}(p)=\left\{q \in M, \phi(q,-t) \in U_{\phi(p,-t)} \forall t \geq 0\right\} .
\end{gathered}
$$

5. If the dynamical system $\phi$ is $C^{k}, k \geq 1$, then the stable and unstable sets are $C^{k}$ submanifolds.

Given a diffeomorphism $f$, the global stable manifold $W^{s}(x)$ where $x$ belongs to a compact hyperbolic set is defined by

$$
W^{s}(x)=\bigcup_{n \geq 0} f^{-n}\left(W_{l o c}^{s}\left(f^{n}(x)\right)\right),
$$


and the global unstable manifold $W^{u}(x)$ is defined by

$$
W^{u}(x)=\bigcup_{n \geq 0} f^{n}\left(W_{l o c}^{u}\left(f^{-n}(x)\right)\right) .
$$

If $\phi_{t}$ is a nonsingular flow with a compact hyperbolic set $A$, the global stable and unstable manifolds at a point $x \in A$ are given by

$$
\begin{aligned}
& W^{s}(x)=\bigcup_{t \geq 0} \phi_{-t}\left(W_{l o c}^{s}\left(\phi_{t}(x)\right)\right), \\
& W^{u}(x)=\bigcup_{t \geq 0} \phi_{t}\left(W_{l o c}^{u}\left(\phi_{-t}(x)\right)\right) .
\end{aligned}
$$

In the case of Anosov systems the dimension of $E^{s}(x)$ is constant, it does not depend on $x$, as well as the dimension of $E^{u}(x)$. Moreover, the subspaces $E^{s}(x)$ depend continuously on $x$, as well as the subspaces $E^{u}(x)$. So Anosov systems have the property that stable sets have constant dimension, and unstable sets have constant dimension too. Since for Anosov diffeomorphisms stable and unstable subspaces have complementary dimensions, we have that stable and unstable manifolds of Anosov diffeomorphisms have a local product structure.

Definition 2.6. Given $k>0,0<m<n, a C^{k}, m$-dimensional foliation of a $C^{\infty} n$-dimensional manifold $M$, is a partition $F$ of $M$ into a collection of sets $F(x)$ called leaves, $x \in M$, satisfying the following properties:

1. $x \in F(x)$ for every $x \in M$, and if $F(x) \cap F(y) \neq \emptyset$ then $F(x)=F(y)$.

2. Given $x \in M$ there exists an open neighborhood $U$ of $x$ and $a C^{k}$ diffeomorphism $\psi:(-1,1)^{m} \times(-1,1)^{n-m} \longrightarrow U$ such that $\psi\left((-1,1)^{m} \times\right.$ $\left.\left\{y_{0}\right\}\right)$ is the connected component of the intersection $F\left(\psi\left(0^{m}, y_{0}\right)\right) \cap U$ which contains $\psi\left(0^{m}, y_{0}\right)$, for every $y_{0} \in(-1,1)^{n-m}$, where $0^{m}$ is the zero vector in $\mathbb{R}^{m}$.

The above definition can be modified to include $C^{0}$ foliations of dimension $m$ : we just replace the $C^{k}$ diffeomorphism $\psi$ by a $C^{0}$ homeomorphism. Similarly, we can define a Hölder foliation, or $C^{\alpha}$ foliation with $\alpha \in(0,1)$, by replacing the $C^{k}$ diffeomorphism $\psi$ by a Hölder homeomorphism with exponent $\alpha$. The map $\psi$ is often called a trivialization of the foliation $F$.

Definition 2.7. Let $F_{1}, F_{2}$ be two $C^{k}$ foliations in a $C^{\infty} n$-dimensional manifold $M$. We say that $F_{1}, F_{2}$ have a $C^{m}$ local product structure, for $m \geq 1$, if for every $x \in M$ there is an open neighborhood $U$ of $x$, and a $C^{m}$ diffeomorphism $\Phi:(-1,1)^{a} \times(-1,1)^{n-a} \longrightarrow U$ such that

1. $\Phi\left((-1,1)^{a} \times\left\{y_{0}\right\}\right)$ is the connected component of $F_{1}\left(\Phi\left(0^{a}, y_{0}\right)\right) \cap U$ containing $\Phi\left(0^{a}, y_{0}\right)$ for every $y_{0} \in(-1,1)^{n-a}$. 
2. $\Phi\left(x_{0} \times(-1,1)^{n-a}\right)$ is the connected component of $F_{2}\left(\Phi\left(x_{0}, 0^{n-a}\right)\right) \cap U$ containing $\Phi\left(x_{0}, 0^{n-a}\right)$ for every $x_{0} \in(-1,1)^{n-a}$.

So $F_{1}, F_{2}$ have local product structure if they have leaves of complementary dimensions and there are local simultaneous trivializations of $F_{1}, F_{2}$ at every point defining local parametrizations of $M$. We can define $C^{0}, C^{\alpha}$ local product structures, with $\alpha \in(0,1)$, replacing the $C^{m}$ diffeomorphism $\Phi$ by a $C^{0}\left(C^{\alpha}\right)$ homeomorphism.

The well known Anosov linear automorphisms of the torus illustrate very well the invariant manifold theorem. The linear map $\tilde{f}: \mathbb{R}^{2} \longrightarrow \mathbb{R}^{2}$, $\tilde{f}(x, y)=(2 x+y, x+y)$ is a symmetric, measure preserving map which preserves as well the lattice of points with integer coordinates. So by means of the covering map from $\mathbb{R}^{2}$ onto $T^{2}$ we can project $\tilde{f}$ into a diffeomorphism $f: T^{2} \longrightarrow T^{2}$. Its differential at every point has two eigenvalues $\lambda<1<\mu$, which yields that $f$ is Anosov. The eigenvectors of $D_{p} f$ determine the directions of the stable and unstable subspaces at $p$, and the eigenvalues give the contraction of stable vectors and the expansion of unstable vectors under the action of $D_{p} f$. The topological theory of Anosov diffeomorphisms is well developed by now. For instance, Anosov diffeomorphisms in $T^{n}$ are conjugated to linear Anosov maps [38], and there is a beautiful theory of the so-called codimension one Anosov diffeomorphism combining dynamics, foliation theory and topology to classify such systems. For a complete survey about the theory of Anosov diffeomorphisms we refer to [101].

The local product structure for invariant submanifolds of flows without singularities is defined in a slightly different way since the stable and the unstable subspaces are not transversal in this case.

Definition 2.8. Let $\psi_{t}: N \longrightarrow N$ be a $C^{\infty}$, complete flow without singularities acting on a $C^{\infty}, n$-dimensional manifold $N$. Suppose that there exists a pair of foliations of $N, \mathcal{F}_{1}, \mathcal{F}_{2}$ preserved by $\psi_{t}$ (i.e., the leaf $\mathcal{F}_{i}(p)$ of $\mathcal{F}_{i}$ containing $p$ satisfies $\psi_{t}\left(\mathcal{F}_{i}(p)\right)=\mathcal{F}_{i}\left(\psi_{t}(p)\right)$ for every $\left.t \in \mathbb{R}\right)$. We say that $\mathcal{F}_{1}, \mathcal{F}_{2}$ have a $C^{k}$ local product structure if for every $p \in N$ there is a local transversal section $S_{p}$ of the flow containing $p, a C^{k}$ diffeomorphism $f:(-1,1)^{(n-1)} \longrightarrow S_{p}$, and integers $a, b$ with $a+b+1=n$, such that

1. $f\left((-1,1)^{a} \times\left\{\overline{y_{0}}\right\}\right)$ is a subset of the connected component of

$$
\mathcal{F}_{1}\left(f\left(0^{a}, \overline{y_{0}}\right)\right) \cap S_{p}
$$

containing $f\left(0, \overline{y_{0}}\right)$ for every $\overline{y_{0}} \in(-1,1)^{b}$.

2. $f\left(\left\{\overline{x_{0}}\right\} \times(-1,1)^{b}\right)$ is a subset of the connected component of

$$
\mathcal{F}_{2}\left(f\left(\overline{x_{0}}, 0^{b}\right)\right) \cap S_{p}
$$

containing $f\left(\overline{x_{0}}, 0\right)$ for every $\overline{x_{0}} \in(-1,1)^{a}$. 
The foliations have a $C^{0}$ local product structure if the local trivialization $f:(-1,1)^{(n-1)} \longrightarrow S_{p}$ is a homeomorphism for every $p \in N$. The foliations have a $C^{\alpha}$ local product structure, where $\alpha \in(0,1)$, if the local trivialization is Hölder with exponent $\alpha$.

Anosov flows have always a pair of foliations with Hölder local product structure, namely, the so-called center stable and center unstable foliations. The center stable foliation $W^{c s}$ of an Anosov flow $\psi_{t}$ is given by the union of the leaves $W^{c s}(p)$ which are defined by

$$
W^{c s}(p)=\bigcup_{t \in R} \phi_{t}\left(W^{s}(p)\right)
$$

where $W^{s}(p)$ is the stable submanifold of $p$. The center unstable foliation $W^{c u}$ of $\psi_{t}$ is given by the union of the leaves $W^{c u}(p)$ which are defined by

$$
W^{c u}(p)=\bigcup_{t \in R} \phi_{t}\left(W^{u}(p)\right),
$$

where $W^{u}(p)$ is the unstable submanifold of $p$. The submanifolds $W^{s}(p)$, $W^{u}(p)$ are usually called strong stable and unstable submanifolds respectively.

The combination of Anosov dynamics and the local geometry of invariant submanifolds (namely, local product structure) give rise to two of the most important features of Anosov dynamics: the Anosov closing lemma and the pseudo-orbit tracing property. These two results are crucial for the proof of the structural stability of Anosov systems and motivate the definitions of weak stability which will appear later in the survey. Such properties will be the subject of the next section.

\section{Local product structure, expansiveness and stability}

We start with the definitions of the main notions of stability that will be considered throughout the survey.

Definition 2.9. Let $f: M \longrightarrow M$ be a $C^{k}$ diffeomorphism acting on a $C^{\infty}$ complete manifold $M$. We say that $f$ is $C^{i}$-topologically stable, for $i \leq$ $k$, if there exists an open $C^{i}$ neighborhood $V$ of $f$ such that for every $h \in V$, there exists a semi-conjugacy $\sigma_{h}: M \longrightarrow M: \sigma_{h}$ is a surjective, continuous map satisfying $\sigma_{h} \circ f=h \circ \sigma_{h}$. If $f$ is a continuous homeomorphism we define the $C^{0}$ topological stability of $f$ by taking $i=0$ in the above definition. 
Definition 2.10. $A C^{k}$ diffeomorphism $f: M \longrightarrow M$ acting on a $C^{\infty}$ complete manifold $M$ is called $C^{i}$-structurally stable if it is $C^{i}$ topologically stable and all the semi-conjugacies $\sigma_{h}$ with diffeomorphisms in the open neighborhood $V$ of $f$ are conjugacies, i.e., homeomorphisms. If $f$ is a continuous homeomorphism, we define the $C^{0}$ structural stability by taking $i=0$ in the above definition.

Notice that structural stability is stronger than topological stability. The purpose of the section is to discuss the famous result due to Anosov:

Theorem 2.2. Let $f: M \longrightarrow M$ be an Anosov diffeomorphism acting on a smooth, compact Riemannian manifold $M$. Then $f$ is $C^{1}$ structurally stable.

We shall deduce Theorem 2.2 from a more general result which will be important in forthcoming sections. We first define a more general category of systems which includes Anosov systems.

Definition 2.11. Let $f: X \longrightarrow X$ be a homeomorphism acting on a metric space $(X, d)$. We say that $f$ is expansive if there exists a constant $\epsilon>0$ such that for any given $x, y \in X$ satisfying $d\left(f^{n}(x), f^{n}(y)\right) \leq \epsilon$ for every $n \in \mathbb{Z}$, we have that $x=y$.

For flows the definition of expansiveness is slightly more technical.

Definition 2.12. Let $\psi_{t}: X \longrightarrow X$ be a continuous flow acting on a metric space $(X, d)$. The flow $\psi_{t}$ is said to be expansive if there exists a constant $\epsilon>0$ such that for every $x \in X$ we have the following property: if for a given $y \in X$ there exists a continuous, surjective map $h: R \longrightarrow R$ with $h(0)=0$ such that

$$
d\left(\psi_{t}(x), \psi_{h(t)}(y)\right) \leq \epsilon
$$

for every $t \in R$ then there exists $t_{0}$ such that $\psi_{t_{0}}(x)=y$.

We shall often call the number $\epsilon$ in the above definitions a expansiveness constant for the dynamical system considered. Observe that if $\epsilon$ is a expansiveness constant then $\epsilon^{\prime} \leq \epsilon$ is a expansiveness constant too. One of the main consequences of Theorem 2.1 is

Corollary 2.1. Every Anosov system acting on a compact Riemannian manifold is expansive.

The proof of this corollary is very simple but illustrates the power of the geometric realization of hyperbolic invariant subspaces given by Theorem 2.1. We just give an outline of the proof in the case of diffeomorphisms for the sake of completeness.

Theorem 2.1 and the local product structure of invariant foliations imply that the dynamics is locally very well approximated by an Anosov linear 
dynamics. So let $x, y$ be two points in the manifold and assume that they do not belong to the same orbit. If they are close enough, say in a local product neighborhood of the system, then the local stable manifold of $x$ meets the local unstable manifold of $y$ in a unique point $p$. Therefore, the forward orbits of $p$ and $x$ will approach exponentially fast, while the forward orbits of $p$ and $y$ will get far from each other exponentially fast. Hence, the forward orbits of $x$ and $y$ will get far from each other exponentially fast by the triangle inequality. The same happens with the backward orbits of $x$ and $y$. This shows the expansiveness of Anosov diffeomorphims, a expansiveness constant can be calculated in terms of the size of local product neighborhoods and the expansion-contraction exponents of the dynamics.

Many important properties of the topological dynamics of Anosov systems hold for expansive systems. Our next result is fully proved in [85], but it appears in some other references about expansive systems in low dimensional manifolds (see for instance [62]).

Theorem 2.3. Let $f: M \longrightarrow M$ be an expansive homeomorphism acting on a smooth, compact Riemannian manifold $M$. Suppose that there exists a pair of continuous, invariant foliations $F_{1}, F_{2}$ satisying the following properties:

1. $F_{1}, F_{2}$ have a $C^{0}$ local product structure.

2. $F_{1}(p)$ is the stable set of $p$ for every $p \in M$, namely,

$$
\lim _{n \rightarrow+\infty} d\left(f^{n}(q), f^{n}(p)\right)=0
$$

for every $q \in F_{1}(p)$ and

$$
\lim _{n \rightarrow-\infty} d\left(f^{n}(q), f^{n}(p)\right)=0
$$

for every $q \in F_{2}(p)$.

Then $f$ is $C^{0}$ topologically stable.

One of the main applications of Theorem 2.3 is that it shows the existence of a family of systems which are topological stable but not structurally stable. We shall postpone the discussion of this matter for the moment and concentrate in the proof of the above two theorems.

The key step of the proof of Theorem 2.3 is, as in Anosov's proof of Theorem 2.2 , the so-called shadowing property or pseudo-orbit tracing property.

Definition 2.13. Let $f: M \longrightarrow M$ be a homeomorphism defined in a complete metric space $M$. Given $\alpha>0$, a sequence $\left\{x_{n}\right\}$ of points in $M$ is called a $\alpha$-pseudo-orbit if $d\left(f\left(x_{n}\right), x_{n+1}\right) \leq \alpha$ for every $n \in \mathbb{Z}$. 
For instance, when $M$ is a compact manifold, any orbit of a $\epsilon-C^{k}$ perturbation of $f: M \longrightarrow M$ is a $\alpha(\epsilon)$-pseudo-orbit of $f$, if $\epsilon$ is sufficiently small. The links between the pseudo-orbit tracing property and stability of systems have been considered by many authors since the 1970's, we would like to point out the classical work of Walters [103]. The next result is the main step toward the proof of Theorem 2.3. It is proved in [85] in a more general setting.

Lemma 2.3. Let $f: M \longrightarrow M$ be a $\epsilon$-expansive homeomorphism defined in a compact manifold. Suppose that $f$ satisfies the assumptions of Theorem 2.3. Then there exists $\alpha=\alpha(\epsilon)>0$ such that every $\alpha$-pseudo-orbit of $f$ can be $\frac{\epsilon}{2}$ shadowed by a unique orbit of $f$. Namely, given a $\alpha$-pseudo-orbit $\left\{x_{n}\right\}$, there exists a unique $p \in M$ such that $d\left(f^{n}(p), x_{n}\right) \leq \frac{\epsilon}{2}$ for every $n \in \mathbb{Z}$.

We shall subdivide the proof of Lemma 2.3 in several steps. Let us begin the proof with the following important remark due to Walters [103].

Lemma 2.4. Let $f: M \longrightarrow M$ be an expansive homeomorphism of a compact manifold $M$ with expansiveness constant $\epsilon>0$. Then given $0<$ $\delta \leq \epsilon$ there exists $N=N(\delta) \in \mathbb{N}$ such that for every pair of points $p, q$ satisfying

$$
\left.\sup \left\{d(p, q), d\left(f^{k}(p), f^{k}(q)\right)\right)\right\} \leq \frac{1}{N},
$$

where $k \in \mathbb{N}$, then we have that

$$
\sup _{0 \leq i \leq k} d\left(f^{i}(p), f^{i}(q)\right) \leq \delta .
$$

Proof. Suppose that the statement is not true. Then there exists some $\delta>0$, a sequence $t_{n}>0$, and pairs of points $p_{n}, q_{n}$, such that

1. $\sup \left\{d\left(p_{n}, q_{n}\right), d\left(f^{t_{n}}\left(p_{n}\right), f^{t_{n}}\left(q_{n}\right)\right)\right\} \leq \frac{1}{n}$,

2. $\sup _{0 \leq i \leq t_{n}} d\left(f^{i}\left(p_{n}\right), f^{i}\left(q_{n}\right)\right) \geq \delta$.

By the continuity of $f$ we can choose $q_{n}$ such that

$$
\sup _{0 \leq i \leq t_{n}} d\left(f^{i}\left(p_{n}\right), f^{i}\left(q_{n}\right)\right)=\delta \leq \epsilon .
$$

Obviously, as $n \rightarrow+\infty$ we have $t_{n} \rightarrow+\infty$. Otherwise, if there exists $T>0$ such that $t_{n} \leq T$, by the uniform continuity of $f$ in $M$ we have that $d\left(p_{n}, q_{n}\right) \leq \frac{1}{n} \rightarrow 0$ implies that $\sup _{0 \leq i \leq T} d\left(f^{i}\left(p_{n}\right), f^{i}\left(q_{n}\right)\right) \rightarrow 0$, which contradicts the choice of $\delta$.

Let $s_{n}$ be such that $d\left(f^{s_{n}}\left(p_{n}\right), f^{s_{n}}\left(q_{n}\right)\right)=\delta$. The above argument shows that $s_{n} \rightarrow+\infty$ and $t_{n}-s_{n} \rightarrow+\infty$ as $n \rightarrow \infty$. 
Consider the pairs of points $x_{n}=f^{s_{n}}\left(p_{n}\right), y_{n}=f^{s_{n}}\left(q_{n}\right)$. By taking a convergent subsequence of such pairs, we get a pair of points $x_{\infty}, y_{\infty}$ such that

1. $d\left(x_{\infty}, y_{\infty}\right)=\delta \leq \epsilon$,

2. $d\left(f^{i}\left(x_{\infty}\right), f^{i}\left(y_{\infty}\right)\right) \leq \delta \leq \epsilon$, for every $i \in \mathbb{Z}$.

This clearly contradicts the expansiveness assumption.

Lemma 2.5. Let $\rho>0$ be the injectivity radius of $M$. Then, given $\delta>0$ there exists $n_{\delta}>0$ such that for every $p \in M$, and every $q$ in the connected component of $F_{1}(p) \cap B_{\rho}(p)$ which contains $p$ we have that $d\left(f^{m}(p), f^{m}(q)\right) \leq \delta$ for every $m \geq n_{\delta}$, where $B_{r}(p)$ is the ball of radius $r$ centered at $p$. Analogously, there exists $m_{\delta}<0$ such that if $q$ is in the connected component of $F_{2}(p) \cap B_{\rho}(p)$ which contains $p$ then $d\left(f^{n}(p), f^{n}(q)\right) \leq \delta$ for every $n \leq m_{\delta}$.

Proof. In other words, the stable sets $F_{1}(p)$ are uniformly contracted by positive iterates of $f$, and the unstable sets $F_{2}(p)$ are uniformly contracted by negative iterates of $f$. The connected component of $F_{1}(p) \cap B_{\rho}(p)$ which contains $p$ plays the role of local stable sets in the case of Anosov diffeomorphisms. In Anosov dynamics, the above contractions are exponential functions of $n$. The choice of $\rho$ as the radius of balls is just to grant that $B_{\rho}(p)$ is embedded for every $p \in M$.

The proof follows from the assumptions of items (1) and (2) in Theorem 2.3 and Lemma 2.4. Let us show for instance that stable sets contract uniformly in the sense of Lemma reftopcontraction. Let $\sigma>0$. Since

$$
\lim _{n \rightarrow+\infty} d\left(f^{n}(q), f^{n}(p)\right)=0
$$

for every $q \in F_{1}(p)$, and the set connected component of $F_{1}(p) \cap B_{\rho}(p)$ which contains $p$ is a compact set, there exists $n_{p}>0$ such that for every $q$ in this connected component we have

$$
d\left(f^{n_{p}}(q), f^{n_{p}}(p)\right)<\sigma .
$$

Since the family of connected components of $F_{1}(p) \cap B_{\rho}(p)$ containing $p$ varies continuously with respect to $p$, we have an open neighborhood $U_{p}$ of $p$ where the same inequality holds. By compactness of $M$, we cover $M$ by a finite number of such neighborhoods and thus get a number $n_{0}>0$ such that $d\left(f^{n_{0}}(q), f^{n_{0}}(p)\right)<\sigma$ for every $p \in M$ and $q$ in the connected component of $F_{1}(p) \cap B_{\rho}(p)$ containing $p$. Since $F_{1}$ is an invariant foliation we have shown that for every $p \in M$,

$$
f^{k n_{0}}(q) \in F_{1}\left(f^{k n_{0}}(p)\right) \cap B_{\sigma}\left(f^{k n_{0}}(p)\right)
$$


for every $k \in \mathbb{N}$ and every $q$ in the connected component of $F_{1}(p) \cap B_{\rho}(p)$ containing $p$. By Lemma 2.4 there exists $\delta(\sigma)$, with $\lim _{\sigma \rightarrow 0} \delta(\sigma)=0$, such that

$$
\sup _{i \geq n_{0}} d\left(f^{i}(p), f^{i}(q)\right) \leq \delta(\sigma)
$$

for every $q$ in the connected component of $F_{1}(p) \cap B_{\rho}(p)$ containing $p$. So given $\delta>0$, the lemma follows just choosing $\sigma$ such that $\delta(\sigma)<\delta$.

\section{Proof of Lemma 2.3}

Let $\epsilon>0$ be an expansiveness constant for $f$. Lemma 2.5 allows us to define the sets

$$
\begin{aligned}
& S_{r}(p)=\left\{q \in M, d\left(f^{n}(q), f^{p}\right) \leq r, \forall n \geq 0\right\}, \\
& U_{r}(p)=\left\{q \in M, d\left(f^{n}(q), f^{p}\right) \leq r, \forall n \leq 0\right\} .
\end{aligned}
$$

Notice that $S_{\epsilon}(p) \subset F_{1}(p)$ and $U_{\epsilon}(p) \subset F_{2}(p)$. Because two orbits which satisfy

$$
\alpha \leq d\left(f^{i}(p), f^{i}(q)\right) \leq \epsilon
$$

for every $i \geq 0$ give rise in their $\omega$-limits to two different orbits $O\left(p_{\infty}\right)$, $O\left(q_{\infty}\right)$ such that

$$
d\left(f^{i}\left(p_{\infty}\right), f^{i}\left(q_{\infty}\right)\right) \leq \epsilon,
$$

for every $i \in \mathbb{Z}$ (like in the proof of Lemma 2.5). Therefore, two forward orbits which stay within a distance of at most $\epsilon$ must approach.

Moreover, from the local product structure of the $C^{0}$ foliations $F_{1}, F_{2}$ it is not difficult to show that there exists $\rho>0$ such that if $d(x, y)<\rho$ then $S_{\epsilon / 4}(x) \cap U_{\epsilon / 4}(y) \neq \emptyset$.

To show the pseudo-orbit tracing property of $f$, it is enough to show the same property for $h=f^{N}$ where $N$ is some fixed integer. We now proceed to determine the constant $\alpha$ in the statement in terms of $\epsilon$. Let us first choose some constants:

1. Let $n_{0}>0$ be the minimum of the integers $n \in \mathbb{N}$ such that

(a) $\frac{\epsilon}{4}+\frac{2 \epsilon}{n}<\frac{\epsilon}{2}$,

(b) $S_{\epsilon / 4}(x) \cap U_{\epsilon / 4}(y) \neq \emptyset$ for every $x, y$ with $d(x, y)<\frac{2 \epsilon}{n}$,

(c) If $q \in S_{\epsilon}(p)$ (or in $U_{\epsilon}(p)$ ), and $d(p, q)<\frac{2 \epsilon}{n}$ then $q \in S_{\epsilon / 4}(p)$ $\left(U_{\epsilon / 4}(p)\right.$ respectively).

2. We take $N>0$ such that $f^{k}\left(S_{\epsilon}(p)\right) \subset S_{\epsilon / n_{0}}\left(f^{k}(p)\right)$, and $f^{-k}\left(U_{\epsilon}(p)\right) \subset$ $U_{\epsilon / n_{0}}\left(f^{-k}(p)\right)$ for every $p \in M$ and $k \geq N$. 
Claim: Every $\frac{\epsilon}{n_{0}}$-pseudo-orbit of $h=f^{N}$ can be $\frac{\epsilon}{2}$-traced by an orbit of $h$.

We follow the same steps of the usual proof in the hyperbolic case (see for instance, Proposition 3.6 in Bowen [18]). However, in some steps of the proof the lack of hyperbolicity will make things a little more difficult. So let us consider a $\frac{\epsilon}{n_{0}}$-pseudo-orbit $\left\{x_{n}\right\}$ of $h$. Let us define a sequence $y_{n}$, $n \in \mathbb{N}$, by the following recursive formula:

$$
\begin{aligned}
y_{0} & =x_{0} \\
y_{1} & =U_{\epsilon / 4}\left(h\left(y_{0}\right)\right) \cap S_{\epsilon / 4}\left(x_{1}\right) \\
& \ldots \\
y_{n} & =U_{\epsilon / 4}\left(h\left(y_{n-1}\right)\right) \cap S_{\epsilon / 4}\left(x_{n}\right) .
\end{aligned}
$$

Let us now show that the orbit of $p_{n}=h^{-n}\left(y_{n}\right) \frac{\epsilon}{2}$-shadows the sequence $x_{i}$, for $0 \leq i \leq n$. To see this, we prove first that

$$
d\left(h^{i}\left(p_{n}\right), y_{i}\right)=d\left(h^{i-n}\left(y_{n}\right), y_{i}\right)<\frac{2 \epsilon}{n_{0}}
$$

and that

$$
h^{i}\left(p_{n}\right) \in U_{\epsilon / 2}\left(y_{i}\right),
$$

for every $0 \leq i \leq n$. We make a sort of reverse induction. For $i=n$ we have $h^{i}\left(p_{n}\right)=h^{n}\left(p_{n}\right)=y_{n}$ which satisfies the above two properties. So we assume that both properties hold for $i=k+1$ and let us show that they hold for $i=k$. By the induction assumption we get that $h^{-n+k+1}\left(y_{n}\right) \in$ $U_{\epsilon / 2}\left(y_{k+1}\right)$, and this yields

$$
h^{-n+k}\left(y_{n}\right) \in h^{-1}\left(U_{\epsilon / 2}\left(y_{k+1}\right)\right) \subset U_{\epsilon / n_{0}}\left(h^{-1}\left(y_{k+1}\right)\right),
$$

where the last inclusion comes from the assumption on $n_{0}$. From the construction of the sequence $y_{n}$ we have that $y_{k+1} \in U_{\epsilon / 4}\left(h\left(y_{k}\right)\right) \cap S_{\epsilon / 4}\left(x_{k+1}\right)$, which implies that

$$
h^{-1}\left(y_{k+1}\right) \in h^{-1}\left(U_{\epsilon / 4}\left(h\left(y_{k}\right)\right)\right) \subset U_{\epsilon / n_{0}}\left(y_{k}\right),
$$

again by the choice of $n_{0}$. Thus we get

$$
\begin{aligned}
d\left(h^{-n+k}\left(y_{n}\right), y_{k}\right) & \leq d\left(h^{-n+k}\left(y_{n}\right), h^{-1}\left(y_{k+1}\right)\right)+d\left(h^{-1}\left(y_{k+1}\right), y_{k}\right) \\
& \leq \frac{\epsilon}{n_{0}}+\frac{\epsilon}{n_{0}}=\frac{2 \epsilon}{n_{0}}
\end{aligned}
$$

that is the first part of the induction assumption. At the same time,

$$
h^{-n+k}\left(y_{n}\right) \in U_{\epsilon / n_{0}}\left(h^{-1}\left(y_{k+1}\right)\right) \subset U_{\epsilon}\left(y_{k}\right),
$$


so from the choice of $n_{0}$ we obtain that in fact $h^{-n+k}\left(y_{n}\right) \in U_{\epsilon / 4}\left(y_{k}\right)$. This finishes the proof of the induction assumption.

Using the previous remark we get

$$
\left.d\left(h^{i}\left(p_{n}\right), x_{i}\right)=d\left(h^{i-n}\left(y_{n}\right), x_{i}\right) \leq d\left(h^{i}\left(p_{n}\right), y_{i}\right)+d\left(y_{i}\right), x_{i}\right) \leq \frac{\epsilon}{4}+\frac{2 \epsilon}{n_{0}}<\frac{\epsilon}{2},
$$

since $y_{i} \in S_{\frac{\epsilon}{4}}\left(x_{i}\right)$ and $\frac{2 \epsilon}{n_{0}}<\frac{\epsilon}{4}$ by hypothesis. This finishes the proof of the claim.

To get an orbit of $h$ which $\frac{\epsilon}{2}$-traces the pseudo-orbit $\left\{x_{n}\right\}$, just take a convergent subsequence of the points $p_{n_{k}} \rightarrow p_{\infty}$. The orbit of $p_{\infty}$ is the desired orbit. This orbit is clearly unique since $\epsilon$ is an expansiveness constant for $f$.

We would like to remark that Lemma 2.3 is proved usually in references about symbolic dynamics under hyperbolicity assumptions. There is a natural, well known version of Lemma 2.3 for compact hyperbolic sets of diffeomorphisms where all stable and unstable submanifolds intersect. Isolated, closed hyperbolic sets are called in the literature basic sets in the context of Axiom A diffeomorphisms, and play an important role in the study of the stability of Axiom A systems. We refer to [18] for further details on the subject.

\section{Proof of Theorem 2.3}

We just outline the proof because it is straightforward from Lemma 2.3. Every orbit of a $\delta-C^{0}$ small perturbation $f_{\delta}$ of an expansive homeomorphism $f$ is a $\alpha=\alpha(\delta)$ pseudo-orbit of $f$. So we can choose $\delta$ small in terms of the expansiveness constant $\epsilon$ of $f$ in order to apply Lemma 2.3 to every orbit of $f_{\delta}$. In this way, we define a map

$$
h: M \longrightarrow M, h(x)=x_{0},
$$

where the $f$-orbit of $x_{0} \frac{\epsilon}{2}$-traces the $f_{\delta}$-orbit of $x$ in the sense of Lemma 2.3. It is clear that $h$ is well defined because $x_{0}$ is unique. Indeed, if the orbits of two points $x_{0}, y_{0} \frac{\epsilon}{2}$-trace the orbit of $x$, by the triangular inequality we would get that $d\left(f^{n}\left(x_{0}\right), f^{n}\left(y_{0}\right)\right) \leq \epsilon$ for every $n \in \mathbb{N}$, which by the expansiveness of $f$ yields $x_{0}=y_{0}$. It is easy to show that $h$ is a continuous semi-conjugacy between $f_{\delta}$ and $f$, which shows that $f$ is $C^{0}$-topologically stable.

Corollary 2.2. In the hypotheses of Lemma 2.3, given $0<\epsilon$ there exists a $C^{0}$ neighborhood $V$ of the $\epsilon$-expansive homeomorphism $f$ such that every $\epsilon$-expansive homeomorphism in $V$ is actually conjugate to $f$. 
Proof. This is an immediate consequence of Lemma 2.3. In fact, if $f_{\delta}$ is $\delta$ $C^{0}$ close to $f$ it is semi-conjugate to $f$ and the orbits of $f \frac{\epsilon}{2}$-trace the orbits of $f_{\delta}$ according to Lemma 2.3. But if $f_{\delta}$ is also $\epsilon$-expansive, the triangular inequality implies that two $f_{\delta}$-orbits cannot be traced by the same $f$ orbit (as in the proof of Theorem 2.3). So the semi-conjugacy between $f_{\delta}$ and $f$ is in fact a conjugacy as we wished to show.

Theorem 2.4. An Anosov diffeomorphism $f: M \longrightarrow M$ defined on a compact manifold $M$ is $C^{1}$ structurally stable.

Proof. We just outline the proof of this famous result. We can show that if a diffeomorphism $h: M \longrightarrow M$ is $C^{1}$ sufficiently close to $f$, then $h$ is Anosov (see for instance [48]). Since Anosov diffeomorphims are expansive, we can apply Corollary 2.2 to $h$ to conclude that $h$ is conjugate to $f$. Since this happens in an open $C^{1}$ neighborhood of $f$, then $f$ is $C^{1}$ structurally stable as we claimed.

The structural stability extends to Axiom A diffeomorphisms acting on compact manifolds which satisfy the strong transversality condition: the intersections of the stable and unstable submanifolds is always transversal. This result was proved by Robbin [80], Robinson [81], and De Melo [28] in the 1970's. Lemma 2.3 is not enough to show the structurally stability of Axiom A systems, some extra work is needed to control the dynamics outside the non-wandering set. We refer to the book by Hirsch, Pugh and Shub [51] for a complete proof of this result.

A further, important consequence of Lemma 2.3 is the well known Anosov closing lemma.

Lemma 2.6. Let $f: M \longrightarrow M$ be an $\epsilon$-expansive homeomorphism defined on a compact manifold $M$. Suppose that there exists a pair of continuous foliations $F_{1}, F_{2}$, which are invariant by $f$, satisfying the assumptions of Theorem 2.3. Suppose that the orbit of $p \in M$ satisfies $d\left(p, f^{n}(p)\right)<\alpha(\epsilon)$ for some $n>0$, where $\alpha(\epsilon)$ is defined in Lemma 2.3. Then there exists a periodic point $p_{0}$ of period $n$ such that $d\left(f^{i}(p), f^{i}\left(p_{0}\right)\right) \leq \epsilon$ for every $0 \leq i \leq n$.

Proof. We consider the sequence of points given by

$$
x_{k}=f^{k_{\bmod _{n}}}(p),
$$

where $k_{\text {mod }_{n}}$ is the integer $k$ modulo $n: k=m_{k} n+k_{\bmod _{n}}$. In this way, $x_{m n+k}=x_{k}$ for every integer $m$. By the assumption on $p$, the sequence $\left\{x_{k}\right\}$ is a $\alpha$-pseudo-orbit of $f$, so by Lemma 2.3 there exists an orbit $\left\{f^{k}\left(p_{0}\right), k \in \mathbb{Z}\right\}$ which satisfies $d\left(x_{k}, f^{k}\left(p_{0}\right)\right) \leq \frac{\epsilon}{2}$. The point is that $p_{0}$ 
must be a periodic point of $f$. Because by the choice of $p_{0}$ and the triangular inequality we get

$$
\begin{aligned}
d\left(f^{k+n}\left(p_{0}\right), f^{k}\left(p_{0}\right)\right) & \leq d\left(f^{k+n}\left(p_{0}\right), x_{k+n}\right)+d\left(x_{k+n}, f^{k}\left(p_{0}\right)\right) \\
& \leq \frac{\epsilon}{2}+d\left(x_{k}, f^{k}\left(p_{0}\right)\right) \\
& \leq 2 \frac{\epsilon}{2}=\epsilon .
\end{aligned}
$$

So the orbits of $p_{0}$ and $q=f^{n}\left(p_{0}\right)$ satisfy $d\left(f^{k}(q), f^{k}\left(p_{0}\right)\right) \leq \epsilon$ for every $k \in \mathbb{Z}$ which implies, by the expansiveness of $f$, that $p_{0}=q=f^{n}\left(p_{0}\right)$ as we claimed.

Corollary 2.3. In the hypotheses of Lemma 2.6, if every point of $f$ is non-wandering then the set of periodic orbits is dense in $M$. In particular, if $f$ preserves the Lebesgue measure then the periodic orbits are dense. Moreover, there exists a dense orbit and hence the limit set coincides with the whole manifold.

All the above results can be extended in a natural way to flows without singularities. Let us start with the definitions of the different stability notions already considered.

Definition 2.14. Let $\psi_{t}: M \longrightarrow M$ be a $C^{k}$ flow without singularities acting on a complete Riemannian manifold $M$. The flow $\psi_{t}$ is said to be $C^{i}-$ topologically stable, where $0 \leq i \leq k$, if there exists a $C^{i}$ neighborhood $V$ of the time one map $f=\psi_{1}$ such that for every flow $\sigma_{t}$ with $\sigma_{1} \in V$ we have the following property:

There exists a continuous surjective map $h_{\sigma}: M \longrightarrow M$ such that for every $y \in M$ there exists a continuous, surjective function $\rho_{y}: R \longrightarrow R$, $\rho_{y}(0)=0$ with

$$
h_{\sigma}\left(\psi_{t}(y)\right)=\sigma_{\rho_{y}(t)}\left(h_{\sigma}(y)\right)
$$

for every $t \in R$. If the map $h_{\sigma}$ is a homeomorphism for every $\sigma_{t}$ where $\sigma_{1} \in V$, and $\rho_{y}$ is bijective for every $y \in M$, then the flow $\psi_{t}$ is said to be structurally stable.

There is a well known procedure to reduce the study of flows without singularities acting on compact manifolds to diffeomorphisms. However, the rigorous formulation of this procedure is full of technical details, so we just give an outline of it for the sake of completeness.

We can obtain a fairly good discrete representation of the continuous dynamics of a flow by taking an appropriate finite collection $\Sigma_{i}, i=1,2, . ., n$, of local cross sections of the flow, and considering the Poincaré maps $P_{i, j}: \Sigma_{i} \longrightarrow \Sigma_{j}$ with a natural partial ordering. 
We say that $\Sigma_{i}$ is consecutive to $\Sigma_{j}$ if there exists a point $x \in \Sigma_{j}$ such that $P_{j, i}(x)=\phi_{t(j, i)}(x) \in \Sigma_{i}$, where

$$
0<t(j, i)=\inf _{k \neq j}\left\{t>0, \phi_{t}(x) \in \Sigma_{k}\right\} .
$$

Let $\Sigma=\cup_{i} \Sigma_{i}$, and let $F: \Sigma \longrightarrow \Sigma$ be given by $F(p)=P_{j(p), i(p)}(p)$, where $p \in \Sigma_{j(p)}$ and $\phi_{t(j(p), i(p))}(x) \in \Sigma_{i(p)}$.

The choice of the sections $\Sigma_{i}$ is made in a way that we can describe the trace of each orbit of the flow in $\Sigma$ as an orbit of the map $F$. We would like to warn the reader about the obvious technical problems involved in the formulation of this sort of discrete coding of the dynamics of the flow. These problems are inherent to the Poincaré maps (just to mention some: the map $F$ might not be continuous, the representation of an orbit as an orbit of $F$ might not be unique). We won't enter into much detail about the subject, we refer the reader to some very good references in the literature ([78], [103] for instance).

In this discrete setting, a $\alpha$-pseudo-orbit of the flow $\phi_{t}$ is given by a collection of points $p_{k} \in \Sigma_{i(k)}$, a collection of curves $c_{k}:\left[t_{k}, s_{k}\right] \longrightarrow M$, $k \in \mathbb{Z}$, defined by

$$
c_{k}(t)=\phi_{t-t_{k}}\left(p_{k}\right),
$$

satisfying the following properties:

1. $c_{k}\left(s_{k}\right) \in \Sigma_{i^{\prime}(k)}$, where $i(k) \neq i^{\prime}(k)$.

2. $d\left(c_{k}\left(s_{k}\right), p_{k+1}\right) \leq \alpha$ for every $k \in \mathbb{Z}$.

So a pseudo-orbit of $\phi_{t}$ is given by a sequence $\left\{c_{k}\right\}$ of curves tangent to the flow, whose endpoints form a pseudo-orbit of the map $F$. The definition of pseudo-orbit depends on the chosen system of sections.

All the proofs of the results about stability proven before for diffeomorphims can be carried out for non-singular flows by using this discrete framework. Hence, the pseudo-orbit tracing property and the topological stability of expansive flows with local product structure; as well as the structural stability of Anosov flows, hold independently of the chosen discrete representation of the flow. Notice then that the semi-conjugacy between a non-singular, expansive flow with local product structure and a neighboring system might not be unique. The study of the uniqueness of conjugacies between Anosov systems is part of a very interesting theory associated to cohomological problems of Anosov systems. The discussion of this theory is out of the scope of this book, we refer to [48] for further details on the subject. 


\section{The stability conjecture(s)}

The stability of Anosov systems raised a very natural question: does the stability of a system imply that such system is hyperbolic? The study of this problem was parallel to the study of the stability of Axiom A systems in the 1970's. The first important result in this subject is due to Newhouse $[71]$.

Theorem 2.5. Let $f: M \longrightarrow M$ be a symplectic $C^{4}$ diffeomorphism acting on a compact manifold $M . f$ is structurally stable if and only if $f$ is Anosov.

A symplectic diffeomorphism is a diffeomorphism whose differential preserves a symplectic form, which was defined in the last section of Chapter 1. Symplectic diffeomorphisms enjoy very special dynamical properties, like the preservation of a volume form and the fact that the non-wandering set is the whole manifold (Lemma 2.1). In the proof of Theorem 2.5 we find two of the main steps in the study of any stability problem.

First of all, the differential of a structurally stable diffeomorphism at a periodic point is a hyperbolic linear map. The proof of this fact in the case of symplectic diffeomorphisms is related with the algebraic nature of symplectic linear maps and the Birkhoff's normal form of elliptic periodic points of symplectic maps (see for instance [70], this is why the $C^{4}$ assumption is required). Secondly, given any recurrent point $p$ of the dynamics of $f$ there exists a $C^{1}$ perturbation $\hat{f}$ of $f$ such that $p$ is a periodic point of $\hat{f}$. This remarkable result is known as the $C^{1}$ Closing Lemma, which was proved by Pugh [77] in the 1960's for diffeomorphims and flows, but also holds in the symplectic category.

The characterization of $C^{1}$ structurally stable systems, a problem which was widely known as the stability conjecture, was obtained in the 1980's by Mañé [63], Liao [64] in the two dimensional case, and by Mañé [65] for any dimensions.

Theorem 2.6. A diffeomorphism $f: M \longrightarrow M$ acting on a compact manifold $M$ is $C^{1}$ structurally stable if and only if it is Axiom $A$ and satisfies the strong transversality condition.

The rich, deep theory developed to solve the stability conjecture had (and still has) a tremendous impact in the $C^{1}$ generic theory of dynamical systems. The characterization of $C^{k}$ structurally stable systems for $k>1$ is, as far as we know, an open problem. Unfortunately, we won't discuss the proof of the $C^{1}$ stability conjecture in the present survey. However, the stability conjecture is one of the main sources of inspiration of many problems in topological dynamics of geodesic flows. 
We would like to finish the section with a list of some considerations and questions about stability and hyperbolicity in the realm of geodesic flows, which give us a guideline for the exposition of many of the results in this survey.

First of all, an important part of the methods used in the proof of the $C^{1}$ stability conjecture do not apply to geodesic flows. Because the $C^{1}$ generic theory is much more difficult in the category of geodesic flows than in general flows. For instance, the $C^{1}$ closing lemma is unknown and hence many essential parts of the proof of the stability conjecture do not proceed. One of the main consequences of the closing lemma is that the non-wandering set of structurally stable systems is the closure of the set of hyperbolic periodic orbits. We shall show how to deal with some questions related to this subject assuming that the geodesic flow is expansive.

An interesting question arising from the stability conjecture is the following: what can be said about a system which enjoys a weaker form of stability? By weak stability we mean the shadowing property (pseudoorbits of the system can be traced by true orbits of the system) and/or the topological stability in some $C^{k}$ topology. In some sense, the shadowing property could be regarded as a form of stability that is stronger than the topological stability. Because according to the results in the previous section, the shadowing property is used to get the topological stability of certain expansive systems. However, outside the context of expansive systems it is hard to link the shadowing property with the topological stability. So it makes sense to assume each one of them independently and try to characterize systems enjoying either one or the other.

By Mañé's theorem (Theorem 2.6) a system which enjoys a form of stability that is not structural stability cannot be Axiom A. In the case of symplectic diffeomorphisms, by Newhouse theorem the system cannot be Anosov. We showed that expansive systems with local product structure are topologically stable and have the shadowing property, and we shall see in Chapter 5 that expansive geodesic flows of compact surfaces have local product structure. Moreover, it is not hard to construct examples of expansive geodesic flows which are not Anosov. In the case of general systems enjoying weaker forms of stability there are some partial results by Sakai [93], [94].

Yet, we shall show in Chapters 5 and 6 that the global geometry of the universal covering of compact manifolds without conjugate points and expansive geodesic flows shares many remarkable properties with the global geometry of manifolds with Anosov geodesic flows. The global geometry of the universal covering is closely related with the so-called geometric group theory of the fundamental group of the manifold. In fact, we shall discuss 
in detail, at the end of the survey, the following question: Does a compact manifold without conjugate points and expansive geodesic flow admits a metric of negative curvature?

Based in the above comments, we could expect that geodesic flows which enjoy weaker forms of stability like the shadowing property or the topological stability have hyperbolic global geometry. The main difficulty here is that many important $C^{1}$ tools used to study structural stability cannot be applied to study weaker forms of stability. For instance, it is not true that weakly stable systems in our sense must have hyperbolic periodic orbits, a fact that holds in hyperbolic systems due to the linearization theory of hyperbolic linear maps. Moreover, in the category of geodesic flows this implies that we cannot use curvature estimates and Jacobi fields to study weakly stable systems, because we saw in Chapter 1 that the action of the differential of the geodesic flow is expressed in terms of Jacobi fields. So we have to develop another kind of theory to understand weakly stable systems.

So the purpose of the following chapters is to show what we can do to study the dynamics of the geodesic flow with $C^{1}$ methods (Chapter 3), and then to show some topological methods developed to study dynamics and global geometry of weakly stable systems (from Chapter 4 on). 


\section{Chapter 3}

\section{$C^{1}$ tools to study geometry and dynamics: Jacobi fields, hyperbolicity and global geometry}

In the previous chapter we discussed the stability properties of Anosov systems, one of their most important features. Stability for a certain dynamical system implies roughly that nearby systems behave analogously. This is relevant in mathematical models because a model is usually an aproximation of a real physical phenomenon. Therefore, we have good reasons to look for Anosov systems in conservative dynamics, and in particular in the case of geodesic flows. The main goals of this chapter are, first of all, to discuss how to find sufficient conditions for a geodesic flow to be Anosov; and secondly, to present some of the main consequences of Anosov dynamics in the global geometry and the topology of the manifold. Certainly, the interplay between Anosov dynamics and global hyperbolic geometry is one of the main motivations for some conjectures about weak stability and global geometry that will be considered in the last part of the survey.

The very definition of Anosov systems requires the existence of invariant subbundles, and in the context of geodesic flows this means the existence of special subspaces of Jacobi fields according to Lemma 1.4. This is what we mean by $C^{1}$ methods: special assumptions on either Jacobi fields or sectional curvatures (sign) should imply particular properties of the dynamics of the geodesic flow. Jacobi fields constitute a first order, linear approximation of the geodesic flow, so it is natural to expect that the global geometry of geodesics could be understood from reasonable assumptions on Jacobi fields. 


\section{Negative curvature and Anosov dynamics}

The first result of the chapter is a classical one, it relates manifolds of negative sectional curvatures with Anosov dynamics.

Theorem 3.1. Let $(M, g)$ be a compact Riemannian manifold with negative sectional curvatures. Then the geodesic flow is Anosov.

Theorem 3.1 was proved first by Hedlund [49] for surfaces of constant negative curvature, Hopf [52] extended this result to compact surfaces of negative curvature, and many years later Anosov [2] proved the theorem for any dimension. We shall give a sketch of proof of this theorem using Rauch's comparison theorem, to illustrate how we can get information about the dynamics of the geodesic flow when we know the behavior of Jacobi fields. This proof is a good example of the interplay between geometry, analysis and the theory of ordinary differential equations in the study of the geodesic flow. We shall need to introduce many important basic tools of the theory of manifolds without conjugate points before the proof of Theorem 3.1.

\subsection{Rauch's comparison theorem}

Let us first recall the Rauch's comparison theorem, whose statement was taken from [29], [23].

Theorem 3.2. (Rauch) Let $(\bar{M}, \bar{g})$ be a complete manifold of dimension $n+k$, where $n=\operatorname{dim}(M)$. Let $\gamma$, be a geodesic of $(M, g)$ and $\bar{\gamma}$ be a geodesic of $(\bar{M}, \bar{g})$, and let $J(t), \bar{J}(t)$ be Jacobi fields in $\gamma, \bar{\gamma}$ respectively such that

1. $J(0)=\bar{J}(0)=0$,

2. $g\left(J^{\prime}(0), \gamma^{\prime}(0)\right)=\bar{g}\left(\bar{J}^{\prime}(0), \bar{\gamma}^{\prime}(0)\right)$

3. $\left\|J^{\prime}(0)\right\|_{g}=\left\|\bar{J}^{\prime}(0)\right\|_{\bar{g}}$.

Suppose that $\bar{\gamma}$ has no conjugate points in $[0, l]$ and that for every $v \in$ $T_{\gamma(t)} M, w \in T_{\bar{\gamma}(t)} \bar{M}$, we have

$$
\bar{K}\left(w, \bar{\gamma}^{\prime}(t)\right) \geq K\left(v, \gamma^{\prime}(t)\right),
$$

where $\bar{K}(X, Y)$ is the sectional curvature of $(\bar{M}, \bar{g})$. Then we have that $\|\bar{J}(t)\|_{\bar{g}} \leq\|J(t)\|_{g}$ for every $t \in[0, l]$. Moreover, if there exists a point $t_{0} \in$ $(0, l]$ such that $\left\|\bar{J}\left(t_{0}\right)\right\|_{\bar{g}}=\left\|J\left(t_{0}\right)\right\|_{g}$, then $K\left(J(t), \bar{\gamma}^{\prime}(t)\right)=K\left(\bar{J}(t), \gamma^{\prime}(t)\right)$ for every $t \in\left(0, t_{0}\right]$.

Rauch's comparison theorem is in fact the geometric version of the famous Sturm-Liouville comparison theorems for ordinary, second order differential equations. Actually, the proof of Rauch's comparison theorem for surfaces follows from the classical Sturm-Liouville comparison theorems applied to the Jacobi equation of geodesics. 


\subsection{Asymptotic Jacobi fields and Riccati equation}

The first step towards the proof of Theorem 3.1 is to understand the socalled Green subbundles of the unit tangent bundle of a manifold without conjugate points. The absence of conjugate points in a geodesic allows the construction of asymptotic Jacobi fields. More precisely, let $\gamma(t)$ be a geodesic without conjugate points where the sectional curvatures are uniformly bounded from below by a constant $K_{0}$. Let $V \in T_{\gamma(0)} M$ be perpendicular to $\gamma^{\prime}(0)$, and let $J_{T}(t)$ be the Jacobi field whose boundary conditions are $J_{T}(0)=V, J_{T}(T)=0$. Since there are no conjugate points in $\gamma$, we have that $J_{T}$ exists and is unique. Moreover, by the equation

$$
g_{\gamma(t)}\left(J(t), \gamma^{\prime}(t)\right)=g_{\gamma(0)}\left(J(0), \gamma^{\prime}(0)\right)+g_{\gamma(0)}\left(J^{\prime}(0), \gamma^{\prime}(0)\right) t,
$$

already used in the proof of Lemma 1.5 we have that the linear function $f(t)=g_{\gamma(t)}\left(J_{T}(t), \gamma^{\prime}(t)\right)$ has two zeroes: $t=0$ and $t=T$. So $f(t)$ must be zero for every $t \in \mathbb{R}$ and hence, $J_{T}(t)$ is perpendicular to $\gamma^{\prime}(t)$ for every $t \in \mathbb{R}$.

Let $e_{0}(t)=\gamma^{\prime}(t), e_{2}(t), . ., e_{n-1}(t)$ be an orthonormal, parallel frame defined along $\gamma(t)$. We can write every Jacobi field $J(t)$ perpendicular to $\gamma$ as

$$
J(t)=\sum_{i=1}^{n-1} g_{\gamma(t)}\left(J(t), e_{i}(t)\right) e_{i}(t) .
$$

Define the curvature matrix $\mathbb{K}(\gamma(t))$ by

$$
\mathbb{K}(\gamma(t))_{i j}=g_{\gamma(t)}\left(R\left(\gamma^{\prime}(t), e_{i}(t)\right) \gamma^{\prime}(t), e_{j}(t)\right),
$$

where $R$ is the curvature tensor of $g$. The Jacobi equation written in the coordinates defined by the frame $\left\{e_{i}(t), i=1,2, . ., n-1\right\}$ gives a matrix Jacobi equation

$$
\mathbb{J}^{\prime \prime}(t)+\mathbb{K}(\gamma(t)) \mathbb{J}(t)=0,
$$

whose solutions are $(n-1) \times(n-1)$ matrices $\mathbb{J}(t)$ obtained as follows: if $J_{1}(t), J_{2}(t), . ., J_{n-1}(t)$ are Jacobi fields which are perpendicular to $\gamma$, then $\mathbb{J}_{i j}(t)=g_{\gamma(t)}\left(J_{i}(t), e_{j}\right)$. Every Jacobi field $J(t)$ perpendicular to $\gamma$ in the subspace generated by $J_{1}(t), J_{2}(t), \ldots, J_{n-1}(t)$ can be written as

$$
J(t)=\mathbb{J}(t) W,
$$

where $W$ is a $(n-1)$ vector. If we consider the Jacobi fields $J_{i, T}(t)$, $i=1,2, \ldots, n-1$, which are perpendicular to $\gamma$ and satisfy $J_{i, T}(0)=e_{i}(0)$, $J_{i, T}(T)=0 \forall i=1,2, . ., n-1$, we get a special matrix solution of the Jacobi equation which shall be denoted by $\mathbb{J}_{T}(t)$. Observe that $\mathbb{J}_{T}(t)$ is invertible for every $t \neq T$, because the Jacobi fields $J_{i, T}(t), i=1,2, . ., n-1$ generate a subspace of dimension $(n-1)$ of Jacobi fields. 
The matrix Jacobi equation gives rise to a matrix Riccati equation

$$
U^{\prime}(t)+U^{2}(t)+\mathbb{K}(\gamma(t))=0
$$

where $U(t)=\mathbb{J}^{\prime}(t) \mathbb{J}(t)^{-1}$. We denote by $U_{T}(t)$, for $T \in \mathbb{R}$, the special solution $U_{T}(t)=\mathbb{J}_{T}^{\prime}(t) \mathbb{J}_{T}(t)^{-1}$, that is defined for every $t \neq T$.

Notice that in the case of surfaces, the above equation is one dimensional, and the curvature matrix is just the Gaussian curvature $K$. Moreover, if the curvature $K$ is a negative constant, we have two constant solutions

$$
U^{s}(t)=-\sqrt{-K}, U^{u}(t)=\sqrt{-K} .
$$

The remaining solutions can be calculated explicitly from the above, and they belong to two types of solutions: the first type are the solutions contained in the strip bounded by $U^{s}$ and $U^{u}$, which are defined for every $t \in \mathbb{R}$; and the second type of solutions are just the functions $U_{T}(t)$, having singularities and defined for every $t \neq T$. Let us denote by $u_{T}^{-}$the solutions which are below $U^{s}$, and $u_{T}^{+}$those which are above $U^{u}$. Then it is easy to check that

$$
\begin{aligned}
& \lim _{T \rightarrow+\infty} u_{T}^{-}(t)=U^{s}(t), \\
& \lim _{T \rightarrow-\infty} u_{T}^{+}(t)=U^{u}(t),
\end{aligned}
$$

where the above limits are uniform on compact subsets. The following lemma was proved by Hopf [53] for surfaces and by Green [45] for any dimension.

Lemma 3.1. Let $(M, g)$ be a compact manifold without conjugate points whose sectional curvatures are bounded below by a constant $-K_{0}$, where $K_{0} \geq 0$. Then there exists a constant $K_{1}$, such that for every geodesic $\gamma$ we have:

1. $\left\|U_{T}(t)\right\|_{\infty} \leq K_{1}$, for every $|t-T| \geq 1$,

2. For every $V \in T_{\gamma(0)} M$ that is perpendicular to $\gamma^{\prime}(0)$, the limit

$$
\lim _{T \rightarrow+\infty} \mathbb{J}_{T}(t)(V)=J_{V}^{s}(t)
$$

exists for every $t \in \mathbb{R}$, and it is a perpendicular Jacobi field with $J_{V}^{s}(0)=V$,

3. Analogously, the limit $\lim _{T \rightarrow-\infty} J_{T}(t)(V)=J_{V}^{u}(t)$ exists, and it is a perpendicular Jacobi field with $J_{V}^{u}(0)=V$,

4. The Jacobi fields $J_{V}^{s}(t), J_{V}^{u}(t)$ never vanish if $V \neq 0$, and we have $\left\|J_{V}^{s \prime}(t)\right\| \leq \sqrt{K_{0}}\left\|J_{V}^{s}(t)\right\|,\left\|J_{V}^{u \prime}(t)\right\| \leq \sqrt{K_{0}}\left\|J_{V}^{u}(t)\right\|$, for every $t \in \mathbb{R}$. 
The main idea of the proof is a comparison argument between the solutions of the Riccati equation of $(M, g)$ along $\gamma$ and the Riccati equation $U^{\prime}(t)+U^{2}(t)-\mathbb{K}_{0}=0$, where $\mathbb{K}_{0}=K_{0} I, I$ is the identity matrix. The proof in the case of surfaces is relatively easy, a complete general proof can be found in [33].

The Jacobi fields $J_{V}^{s}$ are called stable Jacobi fields, the Jacobi fields $J_{V}^{u}$ are called unstable Jacobi fields. It is easy to check that in the case of constant negative curvature $-a^{2}$, the norms of such Jacobi fields satisfy

$$
\begin{gathered}
\left\|J_{V}^{s}(t)\right\|=\|V\| e^{-a t}, \\
\left\|J_{V}^{u}(t)\right\|=\|V\| e^{a t},
\end{gathered}
$$

for every $t \in \mathbb{R}$. Hence, if $(M, g)$ is compact with negative curvature, applying Rauch's comparison theorem we get upper bounds, which are exponential functions, for the norm of stable and unstable Jacobi fields.

The stable and unstable Jacobi fields of the geodesic $\gamma_{\theta}$ give rise to special matrix solutions of the Jacobi equation $\mathbb{J}_{\theta}^{s}(t), \mathbb{J}_{\theta}^{u}(t)$ which are determined by

$$
\mathbb{J}_{\theta}^{s}(t)(V)=J_{V}^{s}(t)
$$

for every $t \in \mathbb{R}$ and $V \in T_{\gamma_{\theta}(0)} M$ perpendicular to $\gamma^{\prime}(0)$;

$$
\mathbb{J}_{\theta}^{u}(t)(V)=J_{V}^{u}(t),
$$

for every $t \in \mathbb{R}$ and $V \in T_{\gamma_{\theta}(0)} M$ perpendicular to $\gamma^{\prime}(0)$. In particular, $\mathbb{J}_{\theta}^{s}(0)=\mathbb{J}_{\theta}^{u}(0)=I$. The corresponding matrix solutions of the Riccati equation

$$
\begin{gathered}
U_{\theta}^{s}(t)=\mathbb{J}_{\theta}^{s \prime}(t)\left(\mathbb{J}_{\theta}^{s}(t)\right)^{-1}, \\
U_{\theta}^{u}(t)=\mathbb{J}_{\theta}^{u \prime}(t)\left(\mathbb{J}_{\theta}^{u}(t)\right)^{-1},
\end{gathered}
$$

will be called respectively the stable Riccati solution and the unstable Riccati solution. By Lemma 3.1, the matrices $U_{\theta}^{s}(t), U_{\theta}^{u}(t)$ are uniformly bounded linear operators for every $\theta \in T_{1} M$, satisfying

$$
\begin{aligned}
& \left\|U_{\theta}^{s}(t)\right\|_{\infty} \leq \sqrt{K_{0}}, \\
& \left\|U_{\theta}^{u}(t)\right\|_{\infty} \leq \sqrt{K_{0}},
\end{aligned}
$$

where $K_{0} \geq 0$ and $-K_{0}$ is a lower bound for the norm of the sectional curvatures of $M$. 


\subsection{Green subbundles}

The set of stable Jacobi fields is a subspace of Jacobi fields, as well as the set of unstable Jacobi fields. These subspaces lift to $T_{(p, v)} T_{1} M$ (Lemma 1.4) to invariant subspaces $E^{s}(p, v), E^{u}(p, v)$ respectively called the Green subspaces, where $(p, v)$ is any point in the orbit $\left(\gamma(t), \gamma^{\prime}(t)\right)$ of the geodesic flow. Green subspaces have dimension $n-1$ if $n=\operatorname{dim}(M)$, and a basis of $E^{s}\left(\phi_{t}(\theta)\right)$ is given by the set

$$
\left\{\left(J_{i}^{s}(t), J_{i}^{s \prime}(t)\right), i=1,2, \ldots, n-1\right\},
$$

where $J_{i}^{s}(t)$ is the stable Jacobi field defined by $J_{i}^{s}(0)=e_{i}(0)$, and $(W, Z) \in$ $N_{\theta}$ is given in coordinates $H_{\theta} \oplus V_{\theta}$. Replacing $s$ by $u$ in the upper indices of $J_{i}^{s}$ we get a basis for $E^{u}(p, v)$.

Let $U_{\theta}^{s}(t), U_{\theta}^{u}(t)$ be the solutions of the Riccati equation defined in the previous subsection along the geodesic $\gamma_{\theta}, \theta=(p, v)$. Then the Green subspaces at $\theta$ are defined by

$$
\begin{aligned}
& E^{s}(\theta)=\left\{\left(W, U_{\theta}^{s}(0) W\right), W \in H_{\theta}\right\}, \\
& E^{u}(\theta)=\left\{\left(W, U_{\theta}^{u}(0) W\right), W \in H_{\theta}\right\},
\end{aligned}
$$

where $H_{\theta}$ is the horizontal subspace of $N_{\theta}$. So Green subspaces are graphs of linear maps which depend on $\theta$. In fact, the dependence of $U_{\theta}^{s}(0), U_{\theta}^{u}(0)$ with respect to $\theta$ is just measurable, and there are examples of compact surfaces without conjugate points where these subspaces do not depend continuously on $\theta[6]$.

The following assertions are easy to check.

1. If the sectional curvatures of $(M, g)$ are nonpositive, the Green subspaces depend continuously on $\theta \in T_{1} M$. Moreover, the Green subspaces attached to a geodesic $\gamma$ are linearly dependent if and only if there exist parallel Jacobi fields along $\gamma$ which are perpendicular to $\gamma$. This implies that there exists a vanishing sectional curvature at every point of the geodesic.

2. If $\operatorname{dim}(M)=2$, we have the following identities:

$$
\begin{gathered}
\left\|J_{\theta}^{s}(t)\right\|=\left\|J_{\theta}^{s}(0)\right\| e^{\int_{0}^{t} U_{\theta}^{s}(r) d r}, \\
\left\|J_{\theta}^{u}(t)\right\|=\left\|J_{\theta}^{u}(0)\right\| e^{\int_{0}^{t} U_{\theta}^{u}(r) d r} .
\end{gathered}
$$

3. If $\operatorname{dim}(M)=2$ and $M$ is compact with negative curvature, the geodesic flow is Anosov. 
Item (3) is a particular case of Anosov's theorem. In the case of surfaces it is easy to verify that negative curvature implies that the stable solutions of the Riccati equation are always negative, and the unstable solutions of the Riccati equation are always positive. This is because the norms of Jacobi fields are strictly convex functions. Now, from item (2) and Lemma 1.4 we get that stable vector are exponentially contracted by $D \phi_{t}, t>0$, and unstable vectors are exponentially contracted if $t<0$.

In higher dimensions, it is also true that negative sectional curvatures imply that the stable and unstable Riccati solutions are respectively, negative definite and positive definite matrices. However, we won't use this approach to show Anosov's theorem in the next section, we shall apply Rauch comparison theorem. If we do not assume any restrictions on the curvature of $(M, g)$, it is more difficult to establish a link between $U_{\theta}^{s}(t)$, $U_{\theta}^{u}(t)$ and the action of $D_{\theta} \phi_{t}$. What we can say is that they are related with the so-called Lyapunov exponents of the geodesic flow. Freire and Mañé [40] show that the metric entropy of the geodesic flow of compact manifolds without conjugate points is given by the integral of the trace of the unstable Riccati operator. We won't give further details on the subject, we suggest the reader to look at [40].

\subsection{Proof of Anosov's theorem and the impact of hy- perbolicity in the global geometry of geodesics}

So let $(M, g)$ be a compact manifold with negative sectional curvatures bounded below by $-K_{0}$, and bounded above by $-a^{2}$, where $a, K_{0}>0$. By Lemma 3.1 we have that for every stable Jacobi field $J^{s}(t)$ and every unstable Jacobi field $J^{u}(t)$,

$$
\begin{gathered}
\left\|J^{s \prime}(t)\right\| \leq \sqrt{K_{0}}\left\|J^{s}(t)\right\|, \\
\left\|J^{u \prime}(t)\right\| \leq \sqrt{K_{0}}\left\|J^{u}(t)\right\| .
\end{gathered}
$$

Let $W^{s}=\left(X_{1}, X_{2}\right) \in E^{s}(\theta)$ be a stable Green vector, and $W^{u}=\left(Z_{1}, Z_{2}\right) \in$ $E^{u}(\theta)$ be an unstable Green vector, where their expression in coordinates correspond to $N_{\theta}=H_{\theta} \oplus V_{\theta}$. Let $J_{\left(X_{1}, X_{2}\right)}^{s}, J_{\left(Z_{1}, Z_{2}\right)}^{u}$ be the associated Jacobi fields according to Lemma 1.4. Then, the same Lemma 1.4 tells us that

where $\|v\|_{S}$ is the Sasaki norm.

$$
\begin{gathered}
\left\|D_{\theta} \phi_{t}\left(W^{s}\right)\right\|_{S} \leq \sqrt{1+K_{0}}\left\|J_{\left(X_{1}, X_{2}\right)}^{s}(t)\right\|, \\
\left\|D_{\theta} \phi_{t}\left(W^{u}\right)\right\|_{S} \leq \sqrt{1+K_{0}}\left\|J_{\left(Z_{1}, Z_{2}\right)}^{u}(t)\right\|,
\end{gathered}
$$

By Rauch's comparison theorem, comparing the Jacobi equation of $(M, g)$ along $\gamma_{\theta}(t)$ with the Jacobi equation of a manifold with constant negative curvature $-a^{2}$, we have that

$$
\left\|D_{\theta} \phi_{t}\left(W^{s}\right)\right\|_{S} \leq\left\|J_{\left(X_{1}, X_{2}\right)}^{s}(0)\right\| \sqrt{1+K_{0}} e^{-a t}
$$


for every $t \geq 0$, and

$$
\left\|D_{\theta} \phi_{t}\left(W^{u}\right)\right\|_{S} \leq\left\|J_{\left(Z_{1}, Z_{2}\right)}^{u}(0)\right\| \sqrt{1+K_{0}} e^{a t},
$$

for every $t \leq 0$. The above inequalities clearly imply that the Anosov conditions are fulfilled by the Green subspaces, thus proving Anosov's theorem.

To finish the section, we would like to mention some of the main consequences of Anosov dynamics in the global geometry of $(M, g)$.

1. Let $p \in \tilde{M}$, and let $S_{t}(p)$ be the sphere of radius $t$ centered at $p$. Given two different geodesic rays $\gamma(t), \beta(t)$ with $\gamma(0)=\beta(0)=p$, the distance in $S_{t}(p)$ between $\gamma(t)$ and $\beta(t)$ diverges exponentially with $t$.

2. The volume growth of $S_{t}(p)$, namely, $\lim _{t \rightarrow+\infty} \frac{1}{t} \ln \left(\operatorname{Vol}\left(S_{t}(p)\right)\right)$ is bounded below by a positive constant independent of $p$.

The proofs of items (1) and (2) follow from the definition of the exponential map and its relationship with radial Jacobi fields, we give a sketch for the sake of completeness. In fact, by Lemma 1.2 we can write the norm of the differential of $\exp _{p}: T_{p} \tilde{M} \longrightarrow \tilde{M}$ in terms of the norm of Jacobi fields which vanish at $p$. But by the Anosov properties of Green subbundles, such Jacobi fields behave asymptotically like unstable Jacobi fields whose norms diverge exponentially. We leave the details to the reader. In forthcoming sections we shall mention other further implications of the Anosov dynamics in the global geometry of $M$.

\section{Characterization of Anosov geodesic flows in manifolds without conjugate points}

In this section we sketch the proof of one of the most important results of the theory of manifolds without conjugate points, which was proved in the early 1970's and is due to Eberlein [33]. As we showed in the previous section, the Green subspaces of a compact, n-dimensional manifold with negative curvature are linearly independent, have dimension $\mathrm{n}-1$, and the differential of the geodesic flow acts hyperbolically when restricted to each one of them. Eberlein's beautiful idea was to show that just the linear independence of Green subspaces characterizes Anosov geodesic flows. About 10 years later, R. Mañé suggested a different point of view to show Eberlein's theorem, using the notion of quasi-Anosov systems ([63] for instance for the definition) that appears in his works about the stability conjecture. We present here a rather simple proof inspired by Mañé's point of view, although we won't use the notion of domination in the argument. 
Theorem 3.3. Let $(M, g)$ be a compact Riemannian manifold without conjugate points. The geodesic flow is Anosov if and only if the Green subspaces are linearly independent at every point.

We shall subdivide the proof in several steps.

\subsection{On the divergence of Jacobi fields in manifolds without conjugate points}

The first one is a fundamental result proved by Eberlein [33] about the divergence of Jacobi fields with a zero in manifolds without conjugate points.

Proposition 3.1. Let $(M, g)$ be compact without conjugate points, and let $\gamma_{\theta}(t)$ be a geodesic, where $\theta \in T_{1} M$. Let $J(t)$ a nontrivial Jacobi field such that $J(0)=0$. Given $c>0, a>0$, there exists $T=T\left(c, a, \gamma_{\theta}\right)>0$ such that if $\left\|J^{\prime}(0)\right\| \geq a$, then $\|J(t)\| \geq c$ for every $t \geq T$.

Proof. We shall sketch the proof in the case of surfaces, which was proved by Green [44], just to give an idea of the method. The main tool of the proof is the Riccati equation, that is easier to handle in dimension 2. The generalization of the argument for dimension $n$ is quite technical and we refer to Eberlein's paper for the complete proof.

So let us suppose that $\operatorname{dim}(M)=2$, where we can restrict ourselves to the study of the one dimensional Jacobi equation along $\gamma_{\theta}(t), f^{\prime \prime}(t)+$ $K(t) f(t)=0$, where $K(t)=K\left(\gamma_{\theta}(t)\right)$ is the Gaussian curvature at $\gamma_{\theta}(t)$. Since $(M, g)$ has no conjugate points, Lemma 3.1 implies that there exists at least a solution $Y(t)$ (either the stable or the unstable one, they might coincide) which never vanishes. Let us suppose that $Y(t)>0$ for every $t \in \mathbb{R}$.

Since the set of solutions $J(t)$ of the Jacobi equation which vanish at $t=0$ is a one dimensional subspace, we can assume that $J(0)=0, J^{\prime}(0)=1$ to show the proposition. The solutions $J(t), Y(t)$ are linearly independent, so their Wronskian is a non-zero constant,

$$
W(J(t), Y(t))=J^{\prime}(0) Y(0)-J(0) Y^{\prime}(0)=J^{\prime}(0) Y(0)=Y(0)>0 .
$$

Let us consider the function $h(t)=\frac{J(t)}{Y(t)}$. Its derivative is

$$
h^{\prime}(t)=\frac{1}{Y^{2}(t)}\left(J^{\prime}(t) Y(t)-J(t) Y^{\prime}(t)\right)=\frac{1}{Y^{2}(t)} W(J, Y)>0,
$$

and thus $h(t)$ strictly increasing.

Let us suppose by contradiction that the proposition is false. Then there exists a sequence $x_{n} \rightarrow+\infty$ such that $\lim _{n \rightarrow+\infty} J\left(x_{n}\right)=c \in \mathbb{R}$. Let $a_{n}$ be 
the sequence given by $Y\left(x_{n}\right)=a_{n} J\left(x_{n}\right)$, which is decreasing since $a_{n}=$ $\frac{1}{h\left(x_{n}\right)}$ and $h(t)$ is increasing. In this way we get that $\lim _{n \rightarrow+\infty} a_{n}=a \geq 0$, so let us consider the solution of the Jacobi equation given by

$$
Z(t)=Y(t)-a J(t)
$$

The above solution of the Jacobi equation is linearly independent of $Y(t)$, so the Wronskian $W(Z, Y)=d$ is a non-vanishing constant. Moreover, we have

$$
\lim _{n \rightarrow+\infty} Z\left(x_{n}\right)=0
$$

Claim: $Z(t) \neq 0$ for every $t>0$.

In fact, we have $Z(0)=Y(0)>0$, and $Z\left(x_{n}\right)=Y\left(x_{n}\right)-a J\left(x_{n}\right)$, which implies that

$$
\frac{Z\left(x_{n}\right)}{J\left(x_{n}\right)}=a_{n}-a>0,
$$

so $Z\left(x_{n}\right)>0$ for every $n \in \mathbb{N}$. Thus, if we had some number $t_{0}>0$ such that $Z\left(t_{0}\right)<0$, the solution $Z(t)$ would have at least two zeroes which is impossible by the no conjugate points assumption.

So we get that $Z(t) \geq 0$ for every $t \geq 0$, and if $Z(s)=0$ for some $s>0$, we would have $Z^{\prime}(s)=0=Y^{\prime}(s)-a J^{\prime}(s)$. This implies that $Y(t), J(t)$ satisfy $Y(s)=J(s), Y^{\prime}(s)=J^{\prime}(s)$, which yields $Y(t)=J(t)$ for every $t \in \mathbb{R}$ by the uniqueness of solutions of the Jacobi equation. Since $Y(0)>0$ and $J(0)=0$ this latter identity is a contradiction, so we get that $Z(t)>0$ for every $t \geq 0$ as we claimed.

By Lemma 3.1, the solutions $u_{1}(t)=\frac{Z^{\prime}(t)}{Z(t)}, u_{2}(t)=\frac{Y^{\prime}(t)}{Y(t)}$ of the Riccati equation $u^{\prime}+u^{2}+K=0$ are bounded by a constant $L$ for every $t \geq 1$. If we divide $W(Z, Y)$ by $Z(t)$ we get

$$
\frac{d}{Z(t)}=\frac{W(Z, Y)}{Z(t)}=\frac{Z^{\prime}(t)}{Z(t)} Y(t)-Y^{\prime}(t)=\left(u_{1}(t)-u_{2}(t)\right) Y(t)
$$

Given $\epsilon>0$ let $n_{0}>0$ be such that $\left|J\left(x_{n}\right)-c\right| \leq \epsilon$ for every $n \geq n_{0}$. Taking $t=x_{n}$ with $n \geq n_{0}$ in the above equation we have

$\frac{d}{Z\left(x_{n}\right)}=\left(u_{1}\left(x_{n}\right)-u_{2}\left(x_{n}\right)\right) Y\left(x_{n}\right)=\left(u_{1}\left(x_{n}\right)-u_{2}\left(x_{n}\right)\right) a_{n} J\left(x_{n}\right) \leq 2 L a(c+\epsilon)$.

On the other hand, $\lim _{n \rightarrow+\infty} Z\left(x_{n}\right)=0$, so $\frac{d}{Z\left(x_{n}\right)}$ cannot be bounded. The contradiction arose from assuming that $J\left(x_{n}\right)$ is bounded for every $n>0$, thus finishing the proof of the proposition. 
We would like to remark that the proof for surfaces shows that the number $T$ does not depend on the geodesic $\gamma_{\theta}$. This is very important and was used by Green to show that geodesic rays in surfaces without conjugate points diverge [44]. We shall come back to this issue later.

\subsection{Quasi-Anosov systems}

Let us start with the definition of a quasi-Anosov system.

Definition 3.1. Let $\psi_{t}: N \rightarrow N$ a $C^{\infty}$ flow defined in a complete Riemannian manifold $N$. The system $\psi_{t}$ is called quasi-Anosov if for every non-zero vector $V \in T N$ that is linearly independent from the vector field that is tangent to the flow we get

$$
\sup _{t \in \mathbb{R}}\left\|D \psi_{t}(V)\right\|=+\infty .
$$

Anosov systems are quasi-Anosov, but the converse of this assertion is not true in general. The main result of this subsection is the following:

Lemma 3.2. Let $(M, g)$ be a compact manifold without conjugate points. If the Green bundles are linearly independent then the geodesic flow is quasiAnosov.

The proof of the lemma follows immediately from the following result:

Lemma 3.3. Let $(M, g)$ be a compact manifold without conjugate points. Suppose that there exists a geodesic $\gamma_{\theta}$ and perpendicular Jacobi field $J$ defined in $\gamma$ such that $\|J(t)\| \leq C$ for every $t \geq 0$. Then $J$ is a stable Jacobi field and the vector $\left(J(t), J^{\prime}(t)\right) \in N_{\phi_{t}(\theta)}$ is in $E^{s}\left(\phi_{t}(\theta)\right)$ for every $t \in \mathbb{R}$. Analogously, if $\|J(t)\| \leq C$ for every $t \leq 0$, then $J$ is an unstable vector field.

Proof. Assume that $\|J(t)\| \leq C$ for every $t \geq 0$. Let us consider the Jacobi fields $J_{T}$ defined in Subsection 3.2 by $J_{T}(0)=J(0), J_{T}(T)=0$. We know that that the limit as $T \rightarrow+\infty$ of $J_{T}$ is a stable Jacobi field.

Claim: $\lim _{T \rightarrow+\infty} J_{T}(t)=J(t)$ for every $t \in \mathbb{R}$.

Indeed, consider the Jacobi fields $Y_{T}(t)=J(t)-J_{T}(t)$ which satisfy

$$
Y_{T}(0)=0, \quad\left\|Y_{T}(T)\right\|=\|J(T)\| \leq C .
$$

Applying Proposition 3.1 to $Y_{T}$ we get

$$
\lim _{T \rightarrow+\infty} Y_{T}^{\prime}(0)=0 .
$$

So clearly, $\lim _{T \rightarrow+\infty} J_{T}^{\prime}(0)=J^{\prime}(0)$, and since $J_{T}(0)=J(0)$ for every $T>0$ we have that $J_{T}(t)$ tends uniformly in $t$ to $J(t)$ (by the continuous 
dependence of solutions of the Jacobi equation upon initial conditions). Therefore, $J(t)$ is a stable Jacobi field as we wished to show. The proof for unstable Jacobi fields is completely analogous.

\section{Proof of Lemma 3.2}

The proof of Lemma 3.2 is straightforward from Lemma 3.3. If the geodesic flow is not quasi-Anosov, there exists by Proposition 1.4 a non-zero Jacobi field $J(t)$ defined in some geodesic $\gamma_{\theta}$, that is linearly independent from the geodesic vector field, such that $\|J(t)\| \leq C$ for every $t \in \mathbb{R}$. Since Jacobi fields split into Jacobi fields perpendicular to $\gamma_{\theta}^{\prime}(t)$ and Jacobi fields parallel to $\gamma_{\theta}^{\prime}(t)$, we get a perpendicular Jacobi $J^{\perp}(t)$ field whose norm is uniformly bounded in time. But then Lemma 3.3 implies that $J^{\perp}(t)$ is in the intersection of the set of stable Jacobi fields and the set of unstable Jacobi fields. Hence, the vector $\left(J^{\perp}(0), J^{\perp \prime}(0)\right) \in N_{\theta}$ belongs to $E^{s}(\theta) \cap E^{u}(\theta)=\{0\}$, so $J^{\perp}(0)=0$. Since $J^{\perp}(t)$ is perpendicular to $\gamma_{\theta}^{\prime}(0)$ this yields that $J^{\perp}(t)=0$ for every $t \in \mathbb{R}$, contradicting the hypothesis on $J(t)$.

This result is the fundamental piece of the proof of Theorem 3.3.

\subsection{Quasi-convexity of Jacobi fields and continuity of Green subbundles}

From this subsection, we shall explore the strong consequences of Lemma 3.2 over the geometry of Jacobi fields.

Corollary 3.1. Let $(M, g)$ be a compact manifold without conjugate points whose Green bundles are linearly independent. Then Jacobi fields are quasiconvex. Namely, there exists $L>0$ such that for every geodesic $\gamma \subset M$ and every Jacobi field $J(t)$ defined in $\gamma$ we have

$$
\sup _{t \in[a, b]}\|J(t)\| \leq L \max \{\|J(a)\|,\|J(b)\|\},
$$

for every $a<b$.

Proof. Assume by contradiction that there exist a sequence of geodesics $\gamma_{\theta_{n}}$, perpendicular Jacobi fields $J_{n}$ defined in $\gamma_{\theta_{n}}$, and sequences $a_{n}<b_{n}$ such that

$$
\sup _{t \in\left[a_{n}, b_{n}\right]}\left\|J_{n}(t)\right\| \geq n \max \left\{\left\|J_{n}\left(a_{n}\right)\right\|,\left\|J_{n}\left(b_{n}\right)\right\|\right\} .
$$

Let $t_{n} \in\left[a_{n}, b_{n}\right]$ be the point where $\|J(t)\|$ attains its maximum value. Clearly, $\left|a_{n}-t_{n}\right|$ and $\left|b_{n}-t_{n}\right|$ tend to $\infty$ with $n$. Let

$$
Z_{n}(t)=\frac{1}{\left\|J_{n}\left(t_{n}\right)\right\|} J_{n}\left(t+t_{n}\right),
$$


be Jacobi fields obtained by dividing $J_{n}$ by $\left\|J_{n}\left(t_{n}\right)\right\|$. Each $Z_{n}(t)$ is a Jacobi field of the geodesic $\beta_{n}(t)=\gamma_{\theta_{n}}\left(t+t_{n}\right)$. We have that $\left\|Z_{n}(t)\right\| \leq 1$ for every $t \in\left[a_{n}-t_{n}, b_{n}-t_{n}\right]$, and $\left\|Z_{n}(0)\right\|=1$. Taking a convergent subsequence of the vectors $\left(\beta_{n}(0), \beta_{n}^{\prime}(0)\right), Z_{n}(0)$, we get a limit geodesic $\gamma(t)$ where there is a limit Jacobi field $J(t)$ satisfying $\|J(0)\|=1$, $\|J(t)\| \leq 1$ for every $t \in \mathbb{R}$, contradicting Lemma 3.2.

Corollary 3.2. Let $(M, g)$ be a compact manifold without conjugate points and linearly independent Green subbundles. Then each Green subbundle is continuous, i.e., the maps $\theta \rightarrow E^{s}(\theta), \theta \rightarrow E^{u}(\theta)$, are continuous in the Grassmanian of subspaces of $T M$.

Proof. This is straightforward from the definition of stable and unstable Jacobi fields and quasi-convexity. Let $T>0$, since the Jacobi fields $J_{T}(t)$ satisty $\left\|J_{T}(0)\right\|=1$, and $J_{T}(T)=0$, the quasi-convexity yields that

$$
\left\|J_{T}(t)\right\| \leq L
$$

for every $t \in[0, T]$. Since stable Jacobi fields $J(t)$ with $\|J(0)\|=1$ are limits of the $J_{T}$ as $T \rightarrow+\infty$, we get that $\|J(t)\| \leq L$ for every $t \geq 0$ and every stable Jacobi field $J(t)$. The same holds for unstable Jacobi fields and $t \leq 0$.

The continuity of the map $\theta \rightarrow E^{s}(\theta)$ follows from the above remark: if we take convergent limits of stable Jacobi fields $J_{n}$ such that $\left\|J_{n}(0)\right\|=1$, then its limit will be a Jacobi field $J_{\infty}$ with $\left\|J_{\infty}(0)\right\|=1$ and $\left\|J_{\infty}(t)\right\| \leq L$ for every $t \geq 0$. By Lemma $3.3 J_{\infty}$ is a stable Jacobi field. The same happens with unstable Jacobi fields, thus proving the continuity of both Green subbundles.

\subsection{Hyperbolic behavior of Green subbundles}

In this subsection we finish the proof of Eberlein's Theorem. We begin with the following further consequence of Lemma 3.2.

Lemma 3.4. Let $(M, g)$ be compact without conjugate points such that the Green subbundles are linearly independent. Then, if $V \in E^{s}(\theta)$ we have

$$
\lim _{t \rightarrow+\infty}\left\|D \phi_{t}(V)\right\|=0
$$

and if $W \in E^{u}(\theta)$ we have

$$
\lim _{t \rightarrow-\infty}\left\|D \phi_{t}(V)\right\|=0
$$

The above limits are uniform in $\theta \in T_{1} M$. 
Proof. By Lemma 1.4 in Chapter 1, it is enough to show the statement for stable and unstable Jacobi fields. By Lemma 3.3 we know that $\|J(t)\| \leq$ $L\|J(0)\|$ for every $t \geq 0$ if $J(T)$ is a stable Jacobi field.

So assume by contradiction that $\|J(t)\|$ does not converge to zero if $t \rightarrow+\infty$. Then there is a sequence $t_{n} \rightarrow+\infty$ and a constant $a>0$ such that $\left\|J\left(t_{n}\right)\right\| \geq a$ for every $n \in \mathbb{N}$. Let us define the Jacobi fields

$$
J_{n}(t)=\frac{1}{\left\|J\left(t_{n}\right)\right\|} J\left(t+t_{n}\right),
$$

which are stable Jacobi fields satisfying

$$
\left\|J_{n}(t)\right\| \leq \frac{L}{a}\|J(0)\|
$$

for every $t \geq-t_{n}$, and $\left\|J_{n}(0)\right\|=1$ for every $n \in \mathbb{N}$.

Let $\gamma$ be the geodesic where $J(t)$ is defined, let $\theta_{n}=\left(\gamma\left(t_{n}\right), \gamma^{\prime}\left(t_{n}\right)\right)$, take a convergent subsequence of points $\theta_{n_{k}} \rightarrow \sigma \in T_{1} M$. We get a geodesic $\gamma_{\sigma}$ possessing a Jacobi field $J_{\sigma}(t)$ whose norm is uniformly bounded in $t \in \mathbb{R}$. This contradicts Lemma 3.2 , so the norm of every stable Jacobi field $J(t)$ must tend to zero as $t \rightarrow+\infty$. The same fact holds for unstable Jacobi fields and $t \rightarrow-\infty$.

The following technical result will lead to the proof of the hyperbolicity of Green subbundles.

Lemma 3.5. Let $f: \mathbb{R} \rightarrow \mathbb{R}$ be a continuous, positive function, that is bounded above by $A>0$, and such that

1. $f(t+s) \leq f(t) f(s)$ for every $s, t$.

2. $\lim _{t \rightarrow+\infty} f(t)=0$.

Then there exists constants $C>0, \lambda \in(0,1)$ such that $f(t) \leq C \lambda^{t} f(0)$ for every $t \geq 0$.

Proof. The proof is an elementary calculus exercise. By the hypothesis, there exists $m \in \mathbb{N}$ such that $f(t)<1$ for every $t \geq m$. By item (2) in the hypothesis we get

$$
f(k m) \leq f(m)^{k}
$$

for every $k \in \mathbb{N}$. Thus, if $t \geq 0, t=c m+r$ where $c \in \mathbb{N}$ and $r \in[0, m)$, which implies

$$
f(t) \leq f(\mathrm{~cm}) f(r) \leq f(m)^{c} f(r) .
$$

Let $A$ be an upper bound of $f$, and let $b=f(m)$. Then we have

$$
f(t) \leq A b^{c}=A b^{\frac{t-r}{m}}=A b^{-\frac{r}{m}}\left(b^{\frac{1}{m}}\right)^{t} .
$$

In this way, letting $C=A b^{-\frac{r}{m}}, \lambda=\left(b^{\frac{1}{m}}\right)^{t}$, we get the lemma. 


\section{Proof of Eberlein's Theorem}

Consider the functions

$$
f(t)=\sup _{\theta \in T_{1} M}\left\|\left.D \phi_{t}\right|_{E^{s}(\theta)}\right\|
$$

for $t \geq 0$ and

$$
g(t)=\sup _{\theta \in T_{1} M}\left\|\left.D \phi_{-t}\right|_{E^{u}(\theta)}\right\|
$$

for $t \geq 0$. It is simple to verify the following assertions:

1. Since $D \phi_{t}, t \in \mathbb{R}$, is a family of linear operators, we have $f(t+s) \leq$ $f(t) f(s), g(t+s) \leq g(t) g(s)$, for every $s, t \geq 0$,

2. By the compactness of $M$ and the continuity of $E^{s}(\theta), E^{u}(\theta)$ with respect to $\theta \in T_{1} M$, the functions $f(t), g(t)$ are bounded above by a constant $A>0$ for every $t \geq 0$,

3. Lemma 3.4 tells us that the limit of both $f(t), g(t)$ as $t \rightarrow+\infty$ is 0 .

Therefore, applying Lemma 3.5 to $f$ and $g$ we conclude that the geodesic flow is Anosov.

\section{Further remarks}

Eberlein's Theorem is closely related with a more general result which does not involve any assumption concerning conjugate points.

Theorem 3.4. A symplectic diffeomorphism acting on a compact manifold is Anosov if and only if it is quasi-Anosov.

Theorem 3.4 is due to Mañé, and it is not easily found in the literature. There is a corresponding version for geodesic flows: quasi-Anosov geodesic flows of compact manifolds are Anosov. The main ideas of the proof of this result are similar to the ideas of the proof of Eberlein's theorem, we give next an outline of proof. Of course, the interesting part is that the quasiAnosov property implies the Anosov property. The first step generalizes Corollary 3.1 .

Lemma 3.6. Let $f: X \longrightarrow X$ be a symplectic diffeomorphism acting on a compact manifold $X$. If $f$ is quasi-Anosov there exists $C>0$ such that for every $p \in X, W \in T_{p} X$, we have

$$
\sup _{n \in[0, m]}\left\|D_{p} f^{n}(W)\right\| \leq C \sup \left\{\|W\|,\left\|D_{p} f^{m}(W)\right\|\right\},
$$

for every $m \in \mathbb{N}$. 
The proof is quite similar to the proof of Corollary 3.1. Arguing by contradiction, if we assume that such constant $C$ does not exists then we get a nonzero vector $W_{0} \in T_{p_{0}} X$ such that

$$
\left\|D_{p_{0}} f^{n}\left(W_{0}\right)\right\| \leq 1
$$

for every $n \in \mathbb{N}$. This is forbidden by the quasi-Anosov assumption.

Once we have the quasi-convexity of the action of the differential $D f$, we can follow step by step the proof of Eberlein's theorem.

1. For every point $p \in X$ there exists a stable subspace

$$
E^{s}(p)=\left\{v \in T_{p} X,\left\|D_{p} f^{m}(v)\right\| \leq D\|v\| \forall m \geq 0\right\} .
$$

This follows from the quasi-convexity of $D f$, the quasi-Anosov assumption and the preservation of a symplectic form (good exercise).

2. In fact, the constant $D$ appearing in item (1) does not depend on the point $p$. This implies that the subspaces $E^{s}(p)$ depend continuously on $p$ and that

$$
\lim _{n \rightarrow+\infty}\left\|D_{p} f^{m}(v)\right\|=0
$$

for every $v \in E^{s}(p)$, and every $p \in X$.

3. Applying Lemma 3.5 we get exponential contraction for the action of $D f$ in $E^{s}(p)$.

4. The preservation of a symplectic form by $f$ grants the existence of unstable subspaces, varying continuously and which are exponentially contracted by the backward iterates of $D f$. This implies that $f$ is Anosov.

\section{Anosov dynamics and globally minimizing properties of geodesics: hyperbolicity im- plies the absence of conjugate points}

We would like to finish this chapter with a remarkable theorem due to Klingenberg [57], which completes the characterization of Anosov geodesic flows in compact manifolds.

Theorem 3.5. Let $(M, g)$ be a compact Riemannian manifold whose geodesic flow is Anosov. Then $(M, g)$ has no conjugate points.

This result was later generalized by Mañé [66], proving the following amazing result: 
Theorem 3.6. Let $(M, g)$ be a compact Riemannian manifold such that the geodesic flow preserves a continuous Lagrangian subbundle. Then $(M, g)$ has no conjugate points.

A subspace $\mathcal{L}_{\theta} \subset N_{\theta}$ is called Lagrangian if $\operatorname{dim}\left(\mathcal{L}_{\theta}\right)=n-1$ and $\Omega^{\perp}(V, W)=0$ for every $V, W \in \mathcal{L}_{\theta}$, where $\Omega^{\perp}$ is the canonical two-form of the geodesic flow. The definition of Lagrangian subspaces of a symplectic form is taken from mathematical physics. A subbundle $\mathcal{L}$ of the normal subbundle $\mathcal{N}$ is called Lagrangian if each subspace in $\mathcal{L}$ is Lagrangian with respect to the canonical two-form $\Omega^{\perp}$.

Mañé [66] observed that the stable and unstable subbundles of an Anosov geodesic flow are Lagrangian, we shall show this fact here to give a flavor of the proof of Theorem 3.6.

Lemma 3.7. Assume that the geodesic flow of $(M, g)$ is Anosov. Then the stable and the unstable subbundles of the flow are invariant and Lagrangian.

Proof. Let $W, Z \in T_{\theta} T_{1} M$ be two vectors in the stable subspace of $\theta$. Since the canonical two-form is invariant by the geodesic flow $\phi_{t}$, we have

$$
\Omega^{\perp}\left(D_{\theta} \phi_{t}(W), D_{\theta} \phi_{t}(Z)\right)=\Omega^{\perp}(W, Z)
$$

for every $t \in \mathbb{R}$. Let $C>0, \lambda \in(0,1)$ be the constants in the definition of Anosov flow, so $\left\|D_{\theta} \phi_{t}(Y)\right\| \leq C \lambda^{t}\|Y\|$ for every $t \geq 0$ and every stable vector $Y$. This yields,

$$
\lim _{t \rightarrow+\infty} \Omega^{\perp}\left(D_{\theta} \phi_{t}(W), D_{\theta} \phi_{t}(Z)\right)=0,
$$

and by the invariance of the form $\Omega^{\perp}$ we conclude that $\Omega^{\perp}(W, Z)=0$ for every pair of vectors $W, Z$ in the stable subspace of $\theta$. The same holds for unstable vectors, letting $t \rightarrow-\infty$.

We can show that Green subbundles are Lagrangian too, regardless of any further hypothesis on the dynamics of the geodesic flow.

Lemma 3.8. The Green subbundles in a manifold without conjugate points are invariant, Lagrangian subspaces.

Proof. This follows from the definition of the canonical two-form combined with the definition of Green subbundles. Let $W^{s}=\left(W_{1}, W_{2}\right)$, $Z^{s}=\left(Z_{1}, Z_{2}\right)$ be two stable vectors in $E^{s}(\theta)$, then

$$
\begin{aligned}
\Omega^{\perp}\left(W^{s}, Z^{s}\right)= & g_{\gamma_{\theta}(t)}\left(J_{\left(W_{1}, Z_{1}\right)}(t), J_{\left(W_{2}, Z_{2}\right)}^{\prime}(t)\right) \\
& -g_{\gamma_{\theta}(t)}\left(J_{\left(W_{1}, Z_{1}\right)}^{\prime}(t), J_{\left(W_{2}, Z_{2}\right)}(t)\right),
\end{aligned}
$$


where $J_{\left(W_{i}, Z_{i}\right)}(t)$ is the Jacobi field such that $J_{\left(W_{i}, Z_{i}\right)}(0)=W_{i}, J_{\left(W_{i}, Z_{i}\right)}^{\prime}(0)=$ $Z_{i}$. This is the Wronskian of the solutions $J_{\left(W_{1}, Z_{1}\right)}(t), J_{\left(W_{2}, Z_{2}\right)}(t)$ which is constant. By the definition of $E^{s}(\theta)$, we know that

$$
J_{\left(W_{i}, Z_{i}\right)}(t)=\lim _{T \rightarrow+\infty} J_{i, T}(t),
$$

where $J_{i, T}(t)$ is the Jacobi field perpendicular to $\gamma_{\theta}$ satisfying $J_{i, T}(0)=$ $J_{\left(W_{i}, Z_{i}\right)}(0), J_{i, T}(T)=0$. So we have

$$
g_{\gamma_{\theta}(T)}\left(J_{1, T}(T), J_{2, T}^{\prime}(T)\right)-g_{\gamma_{\theta}(T)}\left(J_{2, T}(T), J_{1, T}^{\prime}(T)\right)=0 .
$$

And since the Wronskian of $J_{1, T}(t), J_{2, T}(t)$ is constant we get that it vanishes for every $t \in \mathbb{R}$. Taking limits as $T \rightarrow+\infty$, the continuity of $\Omega^{\perp}$ yields that $\Omega^{\perp}\left(W^{s}, Z^{s}\right)=0$ for every pair of stable vector fields in $E^{s}(\theta)$. Since the dimension of $E^{s}(\theta)$ is n- 1 , this subspace is Lagrangian for every $\theta$. An analogous argument taking $T \rightarrow-\infty$ shows that $E^{u}(\theta)$ is Lagrangian.

Lemma 3.8 shows that manifolds without conjugate points whose sectional curvatures are bounded from below always have invariant, Lagrangian subspaces. Theorem 3.6 tells us that the converse of the above assertion is also true.

Mañé proved Theorem 3.6 using tools of symplectic topology (Maslov index). Theorem 3.6 belongs to a remarkable research area in mathematical physics and calculus of variations which relates the existence of the so-called invariant graphs of the dynamics with globally minimizing properties of the orbits of the dynamics with respect to some variational principle. The theory of the Hamilton-Jacobi equation, Birkhoff Theorems for twist maps and Lagrangian, invariant tori, and Mather's theory, are also important bodies of work in this research area. The proof of Theorem 3.6 is out of the scope of this survey. We recommend [72] for a complete, clear proof of the theorem.

\subsection{Conclusions}

As a conclusion of the subsection, we get that the geodesic flow of a compact manifold is Anosov if and only if the manifold has no conjugate points and the Green subspaces are linearly independent at every point. So Anosov geodesic flows are well understood from the point of view of the geometry of the manifold. In the forthcoming chapters we shall discuss the influence of Anosov dynamics in the global geometry of the manifold and the structure of the fundamental group.

The structural stability of Anosov flows, which implies that the Anosov dynamics is persistent in open neighborhoods of the flow, has an interesting corollary in the case of geodesic flows. Namely, the persistence of 
Anosov dynamics carries the persistence of the absence of conjugate points of the metric according to Klingenberg's theorem. More precisely, if the geodesic flow of a compact manifold $(M, g)$ is Anosov, then there exists a $C^{2}$ neighborhood $U$ of $(M, g)$ such that the geodesic flow of every metric in the neighborhood $U$ has no conjugate points. The persistence of globally minimizing properties of all geodesics is is quite exceptional in the set of metrics of a manifold, and in fact this remark has a converse proved in [83]:

Theorem 3.7. Let $(M, g)$ be compact without conjugate points. If there exists a $C^{2}$ open neighborhood of $(M, g)$ where the geodesic flow of every metric has no conjugate points, then the geodesic flow is Anosov.

Theorem 3.7 says that the interior in the $C^{2}$ topology of the set of metrics in compact manifolds without conjugate points is precisely the set of metrics whose geodesic flows are Anosov. These metrics are usually called Anosov metrics. An open question is to know whether the boundary of the set of metrics in $M$ without conjugate points is in the closure of Anosov metrics, given that $M$ admits an Anosov metric.

\section{Further applications of the Riccati equa- tion in ergodic theory}

The Riccati equation is a very useful tool in the theory of manifolds without conjugate points. We would like to finish the chapter by mentioning, for the sake of completeness, some of its applications in ergodic theory.

The Riccati equation is closely related with the calculus of the metric entropy of the geodesic flow with respect to the Liouville measure. The definition of the metric entropy of a dynamical system with respect to a measure preserved by the system is quite technical and we won't include it in the survey (we refer to [48] for instance for details). Intuitively, the metric entropy is a way of measuring chaos in a system. Freire and Mañé in [40] showed the following result:

Theorem 3.8. Let $(M, g)$ be a compact manifold without conjugate points. Then the metric entropy $h_{\mu}\left(\phi_{t}\right)$ of the geodesic flow $\phi_{t}$ with respect to the Liouville measure $\mu$ is given by the following formula:

$$
h_{\mu}\left(\phi_{t}\right)=\int_{T_{1} M} \operatorname{trace}\left(U_{\theta}^{u}(0)\right) d \mu,
$$

where $U_{\theta}^{u}(0)$ is the solution of the Riccati equation in the geodesic $\gamma_{\theta}$ associated to the unstable Green subspace $E^{u}(\theta)$, and trace $(M)$ is the trace of a linear operator $M$. 
The proof of Theorem 3.8 uses the fact that the metric entropy is the integral of the positive Lyapunov exponents of the flow, an equation known as Pesin's formula. Recall that the Lyapunov exponent $\chi_{\theta}(W)$ of a tangent vector $W \in T_{\theta} T_{1} M$ is given by

$$
\chi_{\theta}(W)=\lim _{t \rightarrow \pm_{-}^{+}} \frac{1}{t} \ln \left(\left\|D_{\theta} \phi_{t}(W)\right\|\right) .
$$

This limit might not exist, however, Osedelets' Theorem [48] grants the existence of a total measure subset $\Lambda$ of $T_{1} M$ where Lyapunov exponents exist for every tangent vector. Moreover, Oseledets' theorem implies that for every $\theta \in \Lambda$ there exists a decomposition

$$
T_{\theta} T_{1} M=E_{1}(\theta) \oplus E_{2}(\theta) \oplus . . \oplus E_{n(\theta)}(\theta)
$$

by invariant subspaces of the flow such that the Lyapunov exponent $\chi_{\theta}^{i}$ is constant in $E_{i}(\theta)$, and such that $\chi_{\theta}^{1}<\chi_{\theta}^{2}<. .<\chi_{\theta}^{n(\theta)}$.

Let us define the function $\chi^{+}(\theta)$ as the sum of the positive Lyapunov exponents of the subspaces $E_{i}(\theta)$. The well known Pesin's formula applied to geodesic flows implies that

$$
h_{\mu}\left(\phi_{t}\right)=\int_{T_{1} M} \chi^{+}(\theta) d \mu,
$$

where $\mu$ is the Liouville measure. Freire-Mañé show in [40] that for geodesic flows of compact manifolds without conjugate points, $\int_{T_{1} M} \chi^{+}(\theta) d \mu$ is just the integral of the trace of the unstable solution of the Riccati equation.

Just to give some hints of the kind of ideas involved in [40], let us look more carefully to the case when $M$ is a surface. The solutions of the Riccati equation are of the form $U(t)=\frac{f^{\prime}(t)}{f(t)}=\frac{d}{d t}(\ln (f(t))$, where $f(t)$ is a solution of the one dimensional Jacobi equation $f^{\prime \prime}(t)+K(t) f(t)=0$. Of course, the above equation is well defined at the points where $f(t) \neq 0$. Integrating we get

$$
f(t)=f(0) e^{\int_{0}^{t} U(r) d r} .
$$

If $J(t)$ is either a stable or an unstable Jacobi field defined in the geodesic $\gamma(t)$, the one-dimensional solution of the Jacobi equation is given by a non vanishing function $f(t)=g\left(J(t), \gamma^{\prime}(t)\right)$. So in fact, we can suppose that the one-dimensional solutions $f(t)$ in the case of surfaces are the norms of Jacobi fields. So let $f(t)$ be the norm of a stable Jacobi field. We get

$$
\frac{1}{t} \ln (f(t))=\int_{0}^{t} U_{\theta}^{s}(r) d r
$$


and if $f(t)$ is the norm of an unstable Jacobi field we have

$$
\frac{1}{t} \ln (f(t))=\int_{0}^{t} U_{\theta}^{u}(r) d r .
$$

Using Lemma 1.4 we can prove easily that for every stable vector $W \in$ $E^{s}(\theta)$, and $\theta \in \Lambda$, we have

$$
\lim _{t \rightarrow+\infty} \frac{1}{t} \ln \left(\left\|D_{\theta} \phi_{t}(W)\right\|\right) \leq \lim _{t \rightarrow+\infty} \frac{1}{t} \int_{0}^{t} U^{s}\left(\phi_{r}(\theta)\right) d r .
$$

An analogous formula holds for unstable vector fields and $U^{u}$. Let $\chi^{s}(\theta)$, $\chi^{u}(\theta)$ be respectively the stable and unstable Lyapunov exponent in $\theta$. Then, by Birkhoff's ergodic theorem we get

$$
\begin{aligned}
& \int_{T_{1} M} \chi^{s}(\theta) d \mu \leq \int_{T_{1} M} U^{s}(\theta) d \mu, \\
& \int_{T_{1} M} \chi^{u}(\theta) d \mu \leq \int_{T_{1} M} U^{u}(\theta) d \mu .
\end{aligned}
$$

Thus, we see that the Riccati solutions associated to stable and unstable Jacobi fields are connected with Lyapunov exponents. Freire-Mañé show that the above inequalities are actually equalities, and that the positive Lyapunov exponents are precisely those of Green unstable vectors.

Freire-Mañé conjectured, inspired by Theorem 3.8, that the metric entropy of the manifold is zero if and only if the manifold is a flat torus. They proved the conjecture for manifolds without focal points, and G. Knieper [60] showed the conjecture for the so-called manifolds with bounded asymptote, which will be defined in Chapter 4 . 


\section{Chapter 4}

\section{$C^{0}$ tools to study global geometry of geodesics in the universal covering of manifolds without conjugate points}

In the previous chapter, we gave some examples of the use of $C^{1}$ tools to study the geodesic flow: a priori restrictions on the sign of sectional curvatures and Jacobi fields, Sturm-Liouville comparison theorems, Riccati equation. In few words, any information about the local geometry of the manifold carries naturally some information about the dynamics of the geodesic flow. We have dealt so far with the application of $C^{1}$ tools to study Anosov geodesic flows, but in a forthcoming section we shall discuss some important, powerful applications to the theory of manifolds with nonpositive curvature.

The use of $C^{1}$ tools in the general theory of manifolds without conjugate points does not go too far, as in the study of nonpositive curvature geometry. The hypothesis of absence of conjugate points might not imply any particular restriction in the local geometry of the manifold. If no assumptions are made on the curvature it is very difficult to say something about the behavior of Jacobi fields and geodesics. Most of the results of the theory of manifolds without conjugate points obtained by using $C^{1}$ methods and no restrictions on curvatures or Jacobi fields use integrals of the Riccati solutions to connect geometry to topological invariants. We shall discuss Hopf's theorem about tori without conjugate points to illustrate our remark. Such line of reasoning works very well in dimension 2 , but in higher dimensions it is not really fruitful. The proof of Hopf's conjecture by Burago-Ivanov [21] has nothing to do with the Riccati equation.

The purpose of this chapter is to introduce some $C^{0}$ (topological rather 
than differentiable) methods to study the global geometry of geodesics in manifolds without conjugate points. We shall discuss the theory of globally minimizing geodesics of compact surfaces developed by Morse [69], which strongly influenced the theory of visibility manifolds introduced by Eberlein-O'Neil [35], and more recently the theory of Gromov hyperbolic spaces [46]. Such theories give us important guidelines to pursue the study of the global geometry of geodesics without any information about the local geometry. By global geometry of geodesics we mean the geometry of geodesics in the universal covering $\tilde{M}$ endowed with the pullback of the metric $g$ of $M$ by the covering map.

We shall review first some of the fundamental objects of the geometry of manifolds without conjugate points. We shall remind many of the different categories of manifolds without conjugate points studied in the literature in order to explore the knowledge on the global geometry of geodesics without direct assumptions on the sectional curvatures. And we shall compare different important features of such manifolds with their counterparts in the theory of manifolds with nonpositive curvature, where there is a quite complete understanding of the global geometry of geodesics.

\section{Horospheres and Busemann flows in $\tilde{M}$}

Let $\tilde{M}$ be the universal covering of $M$, and let $(\tilde{M}, \tilde{g})$ be the universal covering endowed with the pullback of the metric $g$ in $M$ by the covering map. We always parametrize geodesics by arc length. We shall often call by $[p, q]$ the geodesic segment joining two points in $\tilde{M}$. This geodesic segment is unique, since geodesics in manifolds without conjugate points are globally minimizing. Let us start discussing this phenomenon.

Definition 4.1. Let $(M, g)$ be a complete Riemannian manifold. A geodesic $\gamma \subset M$ is called globally minimizing if for every lift $\tilde{\gamma}$ of $\gamma$ in $(\tilde{M}, \tilde{g})$ we have that $\tilde{\gamma}([s, t])$ is the curve of minimal length joining $\tilde{\gamma}(s)$ and $\tilde{\gamma}(t)$ for every pair of real numbers $s<t$.

Geodesics in Euclidean spaces and manifolds with nonpositive curvature are always globally minimizing. This follows from Lemma 1.2 and the fact that the norm of Jacobi fields is always convex in nonpositive curvature. Indeed, geodesic rays starting at a point $p \in \tilde{M}$ hit a point $q \neq p$ in just one direction, since geodesic rays diverge if the norm of Jacobi fields is convex. So the geodesic $[p, q]$ is unique in $\tilde{M}$ which yields that the length of $[p, q]$ is the minimal length of curves joining $p, q$. Now, if $\theta=(p, v)$ is the unit vector tangent to the geodesic $[p, q]$ at $p$ in a way that $\gamma_{\theta}(t)=q$ for $t=d(p, q)$, the same reasoning shows that $\gamma_{\theta}([s, t])$ is the curve with minimal length joining $\gamma_{\theta}(s)$ and $\gamma_{\theta}(t)$, which yields that geodesics are globally minimizing as we claimed. 
In manifolds without conjugate points, each geodesic has no conjugate points, which is in principle weaker than globally minimizing. In fact, basic Morse theory for geodesics [29] tells us that given a geodesic $\gamma$ without conjugate points, and points $\gamma(s), \gamma(t), s<t$, the length of $\gamma([s, t])$ minimizes the length of local variations of $\gamma([s, t])$ by curves joining $\gamma(s)$ and $\gamma(t)$.

Lemma 4.1. Geodesics in complete manifolds without conjugate points are globally minimizing.

Proof. The proof relies on the non-singularity of the exponential map, as in the case of manifolds with nonpositive curvature. Indeed, once $(M, g)$ has no conjugate points, $(\tilde{M}, \tilde{g})$ has no conjugate points. So the map $\exp _{p}: T_{p} \tilde{M} \longrightarrow \tilde{M}$ is non-singular and hence a covering map for every $p \in \tilde{M}$. Since $\tilde{M}$ is simply connected we have that $\exp _{p}$ is a diffeomorphism, so given a point $q \in \tilde{M}$ different from $p$, there exists a unique geodesic $\exp _{p}(t v)$ reaching $q$. Since the manifold is complete, there exists a minimizing geodesic joining $p$ to $q$, which has to be a subset of $\exp _{p}(t v)$. The same reasoning we used in the case of nonpositive curvature yields that $\exp _{p}(t v)$ is globally minimizing.

The fact that every geodesic is globally minimizing has strong topological consequences. The first one we would like to mention is the existence of the so-called Busemann functions: given $\theta=(p, v) \in T_{1} \tilde{M}$ the Busemann function $b^{\theta}: \tilde{M} \longrightarrow R$ associated to $\theta$ is defined by

$$
b^{\theta}(x)=\lim _{t \rightarrow+\infty}\left(d\left(x, \gamma_{\theta}(t)\right)-t\right)
$$

The level sets of $b^{\theta}$ are the horospheres $H_{\theta}(t)$ where the parameter $t$ means that $\gamma_{\theta}(t) \in H_{\theta}(t)$. Notice that $\gamma_{\theta}(t)$ intersects each level set of $b^{\theta}$ perpendicularly at only one point in $H_{\theta}(t)$. Moreover,

$$
b^{\theta}\left(\gamma_{\theta}(s)\right)=\lim _{t \rightarrow+\infty}\left(d\left(\gamma_{\theta}(s), \gamma_{\theta}(t)\right)-t\right)=\lim _{t \rightarrow+\infty}((t-s)-t)=-s,
$$

for every $s \in \mathbb{R}$. So $b^{\theta}\left(H_{\theta}(s)\right)=-s$ for every $s \in \mathbb{R}$.

We list some basic properties of horospheres and Busemann functions that will be needed in the forthcoming sections (see [75], [26] for instance, for details).

Lemma 4.2. Let $(M, g)$ be a $C^{\infty}$ Riemannian manifold without conjugate points whose sectional curvatures are bounded from below by a constant $C \leq 0$. Then we have:

1. $b^{\theta}$ is a $C^{1}$ function for every $\theta$.

2. The gradient $\nabla b^{\theta}$ has norm equal to one at every point. 
3. Every horosphere is a $C^{1+K}$, embedded submanifold of dimension $n-1$ ( $C^{1+K}$ means $K$-Lipschitz normal vector field), where $K$ is a constant depending on curvature bounds.

4. The orbits of the integral flow of $-\nabla b^{\theta}, \psi_{t}^{\theta}: \tilde{M} \longrightarrow \tilde{M}$, are geodesics which are everywhere perpendicular to the horospheres $H_{\theta}$. In particular, the geodesic $\gamma_{\theta}$ is an orbit of this flow and we have that

$$
\psi_{t}^{\theta}\left(H_{\theta}(s)\right)=H_{\theta}(s+t)
$$

for every $t, s \in R$.

Proof. We would like to give the main lines of the proof for the sake of completeness. The main point of the proof is the bounded geometry of large spheres in $\tilde{M}$ granted by the solutions of the Riccati equation. Observe that the non-singularity of the exponential map at every point of $(\tilde{M}, \tilde{g})$ implies that spheres are $C^{\infty}$, embedded, codimension one submanifolds. Let us consider the sphere $S_{r}(x)$ of radius $r$ centered at $x \in \tilde{M}$.

Claim: The second fundamental form of $S_{r}(x)$ at the point $\exp _{x}(r v)=$ $\gamma_{(x, v)}(r)$ is the product of $(-1)$ by the solution of the Riccati equation (equation 3.1)

$$
U_{\theta(v, r), r}(t)=J_{\theta(v, r), r}^{\prime}(t)\left(J_{\theta(v, r), r}(t)\right)^{-1},
$$

at $t=r$, where $\theta(v, r)=\left(\gamma_{(x, v)}(r),-\gamma_{(x, v)}^{\prime}(r)\right)$, and $J_{\theta(v, r), r}(t)$ is the matrix solution of the Jacobi equation defined by Jacobi fields perpendicular to $\gamma_{\theta(v, r)}$ satisying $J_{\theta(v, r), r}(r)=0$.

The second fundamental form $A_{q}: T_{q} S_{r}(x) \longrightarrow T_{q} S_{r}(x)$ of $S_{r}(x)$ at a point $q$ is the linear operator

$$
A_{q}(Y)=\nabla_{Y}(N)
$$

which gives the covariant derivative of the unit, inward field of normals $N$ of $S_{r}(x)$ at $q$ with respect to $Y$. To show the claim, first observe that the sphere of radius $t>0$ centered at $x$ is given by

$$
S_{t}(x)=\left\{\exp _{x}(t v), v \in T_{x} \tilde{M},\|v\|=1\right\} .
$$

The field of unit normals $N(q)$ at $q \in S_{t}(x)$ is just

$$
N(q)=N\left(\exp _{x}\left(t v_{q}\right)\right)=\theta\left(v_{q}, t\right),
$$

where $q=\exp _{x}\left(t v_{q}\right)$. So to get a unit vector $Y \in T_{q} S_{r}(x)$ we consider a variation of geodesic rays of the form

$$
f(t, s)=\exp _{x}(t v(s))
$$


with $|s|<\epsilon, t=r$, and $v(s)$ a differentiable curve of unit vectors in $T_{x} \tilde{M}$ satisfying $D_{r v(0)} \exp _{x}\left(v^{\prime}(0)\right)=Y$ (this follows from Lemma 1.2). Thus we get

$$
A_{q}(Y)=\nabla_{Y} N=-\nabla_{N} Y=-J_{r}^{\prime}(0)
$$

where $J_{r}(t)=D_{(r-t) v(0)} \exp _{x}\left(t v^{\prime}(0)\right)$ is the Jacobi field defined in $\gamma_{\theta(v, r)}$ (according to Lemma 1.2) satisfying $J_{r}(0)=Y, J_{r}(r)=0$. The equality $\nabla_{Y} N=-\nabla_{N} Y$ comes from

$$
\nabla_{Y} N-\nabla_{N} Y=[Y, N]=0,
$$

where $[Y, N]$ is the Lie bracket of $Y, N(N$ and $Y$ commute because they are coordinate vector fields of the parametrized surface $f(t, s)$ ). Taking an orthonormal basis $\left\{e_{1}, e_{2}, . ., e_{n-1}\right\}$ of $T_{q} S_{r}(x)$, and considering the Jacobi fields $J_{r}^{i}(t)$ in $\gamma_{\theta(v, r)}$ given by $J_{r}^{i}(0)=e_{i}, J_{r}^{i}(r)=0$, we get a matrix solution $J_{\theta(v, r), r}(t)$ of the Jacobi equation defining perpendicular Jacobi fields in $\gamma_{\theta(v, r)}$. The matrix $J_{\theta(v, r), r}(t)$ satisfies $J_{\theta(v, r), r}(0)=I$. By the above considerations about the second fundamental form of spheres we have

$$
A_{q}\left(e_{i}\right)=-J_{r}^{i \prime}(0)=-U_{\theta(v, r)}(0)\left(e_{i}\right),
$$

with $U_{\theta(v, r)}(t)=J_{\theta(v, r), r}^{\prime}(t)\left(J_{\theta(v, r), r}(t)\right)^{-1}$ is the matrix solution of the Riccati equation given in Section 1.2 of Chapter 3. This proves the claim.

To proceed with the proof of the Lemma, let $p \in \tilde{M}$, let $w \in T_{p} \tilde{M}$ be a unit vector, and consider the family of spheres $S_{r}\left(\gamma_{(p, w)}(r)\right), r>0$. This family is monotone, in the sense that the region bounded by $S_{r}\left(\gamma_{(p, w)}(r)\right)$ contains all $S_{t}\left(\gamma_{(p, w)}(t)\right)$ if $t<s$. By the Claim and Lemma 3.1, given $T>0$ there exists $K>0$ such that the norm of the second fundamental form at every point of $S_{r}\left(\gamma_{(p, w)}(r)\right)$ is bounded above by $K$. So by Arzela-Ascoli theorem, given any compact ball $B_{R}(p)$ centered at $p$ there is a convergent subsequence of the sets $S_{r}\left(\gamma_{(p, w)}(r)\right) \cap B_{R}(p)$ in the $C^{1}$ topology. The limit set $\Sigma(p, w)$ is a $C^{1}$ submanifold with $K$-Lipschitz first derivatives.

The geodesic rays starting at $\gamma_{(p, w)}(r)$ converge to a flow if $r \rightarrow+\infty$ which is perpendicular to $\Sigma(p, w)$. And the definition of the Busemann function implies that $\Sigma(p, w)=H_{(p, w)}(0)$. Replacing $p$ by any point $\gamma_{(p, w)}(t)$ we get a horosphere $H_{(p, w)}(t)$ which contains $\gamma_{(p, w)}(t)$. The limit flow of geodesic rays from $\gamma_{(p, w)}(r)$ is perpendicular to every $H_{(p, w)}(t)$. So this flow is just the Busemann flow. The regularity of the Busemann function and flow follows from the regularity of the horospheres. This proves items (1), (2), (3) in the Lemma.

Item (4) follows from the first variation formula (Lemma 1.1) applied to variations by geodesics of $\gamma_{(p, w)}$ joining $\gamma_{(p, w)}(t)$ to points in $H_{(p, w)}(s)$. Indeed, the first variation formula gives that the geodesic of minimal length of the type $\left[\gamma_{(p, w)}(t), q\right]$, where $q \in H_{(p, w)}(s)$, has to be perpendicular to 
$H_{(p, w)}(s)$ at $q$. By uniqueness of geodesics with respect to initial conditions, we get that $q=\gamma_{(p, w)}(s)$ and that the distance from $\gamma_{(p, w)}(t)$ to $H_{(p, w)}(s)$ is $|t-s|$. Thus, we actually have that the horospheres $H_{\theta}(t)$ are equidistant, i.e., given any point $p \in H_{\theta}(s)$, the distance $d\left(p, H_{\theta}(t)\right)$ is equal to $|t-s|$. This proves item (4).

Notice that by item (4) in Lemma 4.1 we have that $b^{\theta}\left(H_{\theta}(t)\right)=-t$ for every $t \in R$. The proof of Lemma 4.2 shows an interesting geometric interpretation of the solutions of the Riccati equation (eq. 3.1 in Chapter 3). The singular solutions of the Riccati equations are the second fundamental forms of spheres. Hence, when the horospheres are of class $C^{2}$ the asymptotic solutions of the Ricatti equation describe the curvatures of the horospheres endowed with the restriction of the metric $\tilde{g}$. In general, although horospheres might not be smooth enough, the asymptotic solutions of the Riccati equation, namely, the stable and the unstable solutions defined in Chapter 3, could be interpreted as their second fundamental forms (they are not true second fundamental forms for the horospheres because ArzelaAscoli theorem in the proof of Lemma 4.2 does not grant $C^{2}$ smoothness of the horospheres).

The link between Riccati solutions and curvatures in spheres and horospheres is very eloquent. We have seen in Chapter 3 that the solutions of the Riccati equation are connected with Lyapunov exponents and contraction (expansion) of spheres and horospheres by the action of the geodesic flow. The relationship with curvatures in spheres shows that the contraction (expansion) produced by the geodesic flow in spheres and horospheres is connected with the way they curve in space. Negative curvature of $(M, g)$ implies that the Riccati solutions are definite linear operators (the stable is negative definite, the unstable is positive definite). This means that spheres and horospheres are convex submanifolds, and Busemann flows tend to either contract horospheres or expand horospheres.

To finish the section, we would like to recall two natural dynamical objects closely related to horospheres: the so-called central subsets of the geodesic flow.

The canonical lift in $T_{1} \tilde{M}$ of $H_{\theta}(0)$ is the set $\tilde{\mathcal{F}}^{s}(\theta)=\left\{\left(p,-\nabla_{p} b^{\theta}\right), p \in\right.$ $\left.H_{\theta}(0)\right\}$. The projection of $\tilde{\mathcal{F}}^{s}(\theta)$ in $T_{1} M$ by the map $P: T_{1} \tilde{M} \longrightarrow T_{1} M$, $P(p, v)=(\Pi(p), D \Pi(v))$, will be denoted by $\mathcal{F}^{s}(P(\theta))$. This set is usually called the stable set of $P(\theta)$, a notation based on the fact that in the case of Anosov geodesic flows $\mathcal{F}^{s}(p, v)$ is indeed the strong stable submanifold of $(p, v)$. The saturation of $\mathcal{F}^{s}(P(\theta))$ by the geodesic flow is called the central stable set of $\theta$. It is not known whether the collection of stable sets forms a continuous foliation. It is true in the case of manifolds of nonpositive curvature, this fact is connected in general with the continuity of horospheres in the compact open topology. 
Analogously, the projection in $T_{1} M$ by the map $P$ of the set $\tilde{\mathcal{F}}^{u}(p, v)=$ $\left\{\left(p, \nabla_{p} b^{(p,-v)}\right), p \in H_{(p,-v)}(0)\right\}$, gives rise to the unstable set $\mathcal{F}^{u}(P(p, v))$ of $P(p, v)$. The saturation by the geodesic flow of an unstable set is called central unstable set. In the case of Anosov geodesic flows, the collection of the sets $\mathcal{F}^{s}(p, v), \mathcal{F}^{u}(p, v),(p, v) \in T_{1} M$ give two continuous foliations $\mathcal{F}^{s}$, the stable foliation, and $\mathcal{F}^{u}$, the unstable foliation. The theory of hyperbolic dynamical systems tells us that the leaves are smooth submanifolds of dimension $n-1$, where $n=\operatorname{dim}(M)$, and that the foliations are Hölder continuous, according to the work of Anosov [2]. In the case of surfaces, the stable and unstable foliations are $C^{1}$, as showed by Hopf [52].

For Anosov flows, by the hyperbolic theory of regularity of invariant submanifolds (Lemma 2.1), the sets $\mathcal{F}^{s}(p, v), \mathcal{F}^{u}(p, v)$ have the same differentiability class of the flow. So horospheres are highly regular too, and in this case the asymptotic solutions of the Riccati equation are in fact the corresponding fundamental forms. Eberlein [34] showed that in manifolds with nonpositive curvature, horospheres are $C^{2}$ submanifolds, providing a non-Anosov example where the Riccati solutions give the second fundamental forms of spheres and horospheres. In the general case of manifolds without conjugate points it is not known if horospheres are always $C^{2}$ submanifolds.

\section{Two-dimensional tori without conjugate points are flat}

The purpose of the next two sections is to survey some of the main results concerning surfaces without conjugate points. We hope to motivate from a geometric point of view many of the conjectures presented and the problems solved at the end of the survey. First of all, the two dimensional sphere has no metric without conjugate points. Because the universal covering of a complete, n-dimensional manifold with a metric without conjugate points is diffeomorphic to $\mathbb{R}^{n}$, and the universal covering of the sphere is the sphere itself. So by the classification of compact surfaces, the topologically simplest surface in the list, after $S^{2}$, is the two-torus $T^{2}$. The following famous result due to E. Hopf [53] solves the issue:

Theorem 4.1. Two dimensional tori without conjugate points are flat.

Proof. The proof is one of the first, paradigmatic examples of the use of the Riccati equation to get information about the global geometry of manifolds without conjugate points. It works very well in dimension two as we already observed.

Let $\left(T^{2}, g\right)$ be a metric without conjugate points. By Lemma 3.1 there exists a solution $U_{\theta}(t)$ of the Riccati equation for every $\theta \in T_{1} M$ which 
never vanishes, we can assume without loss of generality that $U_{\theta}(t)=U_{\theta}^{s}(t)$. The function $\theta \rightarrow U_{\theta}^{s}(0)$ is measurable, and $U_{\phi_{t}(\theta)}^{s}(0)=U_{\theta}^{s}(t)$ for every $t \in \mathbb{R}$. Integrating the Riccati equation and taking the mean value of the integrals in the equation, we get

$$
\lim _{t \rightarrow+\infty} \frac{1}{t} \int_{0}^{t}\left(U_{\theta}^{s}(t)\right)^{2} d t=-\lim _{t \rightarrow+\infty} \frac{1}{t} \int_{0}^{t} K\left(\gamma_{\theta}(t)\right) d t,
$$

where $K(p)$ is the Gaussian curvature at $p$. The above limits exist in a full Lebesgue measure set of $T_{1} M$ by Birkhoff's ergodic theorem (Theorem 1.3). Notice that $\gamma_{\theta}(t)=\gamma_{\phi_{t}(\theta)}(0)$, so the same Birkhoff's theorem implies that

$$
\int_{T_{1} T^{2}}\left(U_{\theta}^{s}(0)\right)^{2} d \mu=-\int_{T_{1} T^{2}} K(\theta) d \mu=-2 \pi \chi\left(T^{2}\right)=0,
$$

where $\chi\left(T^{2}\right)$ is the Euler characteristic of the torus. In the last equality we used of course Gauss-Bonet theorem. Hence, the operator $\left(U_{\theta}^{s}(0)\right)^{2}$ must be equal to zero in a total Lebesgue measure set, because it is positive semi-definite. Since $U_{\theta}^{s}(0)$ is a symmetric linear operator, this yields that $U_{\theta}^{s}(0)=0$ almost everywhere in $T_{1} T^{2}$.

The set $\Gamma$ where $U_{\theta}^{s}(0)=0$ might not be invariant, but since the geodesic flow preserves the Lebesgue measure, we have that $\phi_{t}(\Gamma) \cap \Gamma$ has total measure for every $t \in \mathbb{R}$. Since a countable intersection of full measure sets is a full measure set, we have that

$$
\Gamma_{\infty}=\bigcap_{t \in \mathbb{Q}} \phi_{t}(\Gamma)
$$

has full measure. Observe that $\Gamma_{\infty}$ is $\phi_{t}$-invariant in the set of rational values of the parameter $t$. So $\theta \in \Gamma_{\infty}$ implies that $\phi_{t}(\theta) \in \Gamma_{\infty}$ for every rational $t$ and hence, $U_{\theta}^{s}(t)=0$ for every rational $t$.

Since $U_{\theta}^{s}(t)$ is differentiable in the geodesic $\gamma_{\theta}$, we conclude that $U_{\theta}^{s}(t)=$ 0 for every $t \in \mathbb{R}$. By the Riccati equation, $K\left(\gamma_{\theta}(t)\right)=0$ for every $t \in \mathbb{R}$. Since $\Gamma_{\infty}$ has total measure, we deduce that the Gaussian curvature is zero in a dense subset of $T^{2}$, which implies that the curvature vanishes everywhere.

This beautiful application of the Riccati equation to get the geometric rigidity of two-dimensional tori was generalized by Green [45], who considered n-dimensional tori and replaced the Gaussian curvature by the Ricci curvature. Using the same Hopf's proof Green showed that n-dimensional tori without conjugate points have nonpositive Ricci curvature, which vanishes everywhere if and only if the torus is flat. The problem of proving Hopf's theorem, usually called the Hopf's conjecture, in any dimension, remained open for about 45 years (Hopf's work appeared in the 1940's), until Burago-Ivanov [21] proved the conjecture using totally different methods. 


\section{Surfaces without conjugate points of higher genus, quasi-geodesics and quasi-convexity}

So surfaces without conjugate points and non constant curvature are either non compact or have genus greater than one. Since it was known from Hadamard's theorem that nonpositive curvature implies no conjugate points, many authors looked for examples of such surfaces admitting regions of positive curvature. Gulliver [47] found non compact examples, Ballmann-Brin-Burns [6] give a compact example of a surface with very high genus where the Green subbundles are not continuous; and recently Donnay-Pugh [30] constructed examples of compact embedded surfaces in $\mathbb{R}^{3}$ with Anosov geodesic flows and hence, by Klingenberg's theorem, with no conjugate points.

Thus, there is no geometric classification of compact surfaces without conjugate points and higher genus. All of them admit a metric of constant negative curvature but there are many other metrics without conjugate points.

We start with the classical work of Morse [69] describing completely the qualitative behavior of globally minimizing geodesics of compact surfaces of genus greater than one. This result is crucial to understand many of the conjectures posed in the survey about weak stability of geodesics and global geometry. More precisely, Morse showed the following:

Theorem 4.2. Let $(M, g)$ be a compact surface of genus greater than one, and let $\left(M, g_{0}\right)$ be a hyperbolic metric in $M$ with constant curvature -1 . Then, given $A>0, B>0$ there exists $C>0$ such that every $A, B$ quasigeodesic in $(\tilde{M}, \tilde{g})$ is in the hyperbolic tubular neighborhood of radius $C$ of some geodesic in the hyperbolic plane $\mathbb{H}^{2}=\left(\tilde{M}, \tilde{g}_{0}\right)$.

An $A, B$-quasi-geodesic of $(\tilde{M}, \tilde{g})$ is a continuous rectifiable curve $\alpha:[0,1] \longrightarrow \tilde{M}$ such that

$$
l_{\tilde{g}}(\alpha[t, s]) \leq A d_{\tilde{g}}(\alpha(t), \alpha(s))+B
$$

for every $t, s \in[0,1]$, where $l_{\tilde{g}}$ is the length in the metric $\tilde{g}$. The definition of quasi-geodesic makes sense in more general spaces, called geodesic metric spaces. We shall come back to this point in more detail in forthcoming sections.

Morse Theorem provided one of the first relevant evidences of the influence of the topology of manifolds in the global geometry of geodesics. The uniformization theory of compact surfaces is crucial for the argument, there is no such a theorem for higher dimensional manifolds. The solution of the Poincaré conjecture by Perelmann and its aplications to Thurston's 
geometrization conjecture yield a version of Morse theorem for some compact three dimensional manifolds. We shall come back to this point at the end of the survey. The following very simple result is the starting point of many strong implications of Theorem 4.2.

Lemma 4.3. Let $(M, g),(M, h)$ be two $C^{\infty}$ Riemannian structures in a compact manifold. Then there exists $A>0$ such that every globally minimizing geodesic of $(M, h)$ is a A,0-quasi-geodesic of $(M, g)$.

Proof. This is in fact straightforward from the fact that the two metrics $g$ and $h$ are equivalent: there exists $B>0$ such that

$$
\frac{1}{B^{2}} g_{p}(v, v) \leq h_{p}(v, v) \leq B^{2} g(v, v)
$$

for every $p \in M$ and every tangent vector $v \in T_{p} M$. The equivalence follows from the compactness of $T_{1} M$ and the continuity of the norms of $h$ and $g$ in $T_{1} M$.

Now, let $\gamma \subset(\tilde{M}, \tilde{h})$ be a globally minimizing geodesic for $\tilde{h}$. Then, the distance in the metric $h$ from $\gamma(t)$ to $\gamma(s)$ is

$$
d_{h}(\gamma(t), \gamma(s))=\int_{t}^{s} h\left(\gamma^{\prime}(r), \gamma^{\prime}(r)\right)^{\frac{1}{2}} d r
$$

for every $t<s$, which implies

$$
\begin{aligned}
\frac{1}{B} d_{g}(\gamma(t), \gamma(s)) & \leq \frac{1}{B} \int_{t}^{s} g\left(\gamma^{\prime}(r), \gamma^{\prime}(r)\right)^{\frac{1}{2}} d r \\
& \leq \frac{1}{B} d_{h}(\gamma(t), \gamma(s))
\end{aligned}
$$

Consider a $g$-minimizing geodesic $\beta:[a, b] \longrightarrow \tilde{M}$ such that $\beta(a)=\gamma(t)$, $\beta(b)=\gamma(s)$. Then, interchanging the roles of $h$ and $g$ in the above estimates we get

$$
d_{h}(\gamma(t), \gamma(s)) \leq B \int_{a}^{b} g\left(\beta^{\prime}(r), \beta^{\prime}(r)\right)^{\frac{1}{2}} d r=B d_{g}(\gamma(t), \gamma(s))
$$

Since changing $\gamma$ we cover all pairs of points in $\tilde{M}$, we get the lemma.

Roughly speaking, Theorem 4.2 and Lemma 4.3 imply that the existence of a hyperbolic metric in a surface yields that globally minimizing geodesics of any metric in the surface behave in the same way when lifted to the hyperbolic space. This nice idea can be considered the germ of what is called nowadays the theory of hyperbolic groups, or geometric group theory. 
Next, we proceed to sketch the proof of Theorem 4.2. For this purpose, we recall some basic features of hyperbolic plane geometry. We shall consider the upper half plane model of the hyperbolic space, $\mathbb{H}^{2}$, endowed with the metric $g_{(x, y)}(v, v)=\frac{\langle v, v\rangle}{y^{2}}$, where $\langle v, v\rangle$ is the Euclidean metric. In this model, the geodesics are the vertical straight lines and the circles which are perpendicular to the horizontal axis. We shall denote by $l_{\mathbb{H}^{2}}(c)$ the hyperbolic length of a curve in $\mathbb{H}^{2}$. We need two preliminary elementary lemmas of hyperbolic geometry to show 4.2 .

Lemma 4.4. 1. The tubular neighborhood of radius $r$,

$$
V_{r}(\gamma)=\left\{p \in \mathbb{H}^{2}, d_{\mathbb{H}^{2}}(p, \gamma) \leq d\right\}
$$

of the hyperbolic geodesic $\gamma(t)=(0, t)$ is the cone $C_{\alpha}$ in $\mathbb{H}^{2}$ bounded by the two straight lines $y=\tan (\alpha) x$, with $x>0$, and $y=-\tan (\alpha) x$, with $x<0$, where $0<\alpha<\frac{\pi}{2}$. The distance $r$ from the vertical axis $\gamma(t)$ and the boundary of $C_{\alpha}$ satisfies the following formula:

$$
r=\ln \left(\cot \left(\frac{\alpha}{2}\right)\right) \text {. }
$$

2. Let $0<\rho_{1}<\rho_{2}$. Then the hyperbolic length of the segment $I_{\alpha, \rho_{1}, \rho_{2}} \subset$ $r_{\alpha}^{+}$bounded by the circles $x^{2}+y^{2}=\rho_{1}^{2}, x^{2}+y^{2}=\rho_{2}^{2}$ is

$$
l_{\mathbb{H}^{2}}\left(I_{\alpha, \rho_{1}, \rho_{2}}\right)=\cosh (s(\alpha)) \ln \left(\frac{\rho_{2}}{\rho_{1}}\right) .
$$

Proof. The proof is elementary, we just sketch it for the sake of completeness. We take polar coordinates $(\rho, \theta)$ in the plane and calculate the lengths of the statement in such coordinates. Any circle $x^{2}+y^{2}=r^{2}$ is a hyperbolic geodesic that is perpendicular to the vertical axis. By the first variation formula we have that the distance from a point $p \in\{y=\tan (\alpha) x\}$ with $\rho(p)=r$ to the vertical axis is just the length of the arc of $x^{2}+y^{2}=r^{2}$ bounded by $y=\tan (\alpha) x$ and the vertical axis. So we have

$$
\begin{aligned}
d_{\mathbb{H}^{2}}(p, \gamma) & =l_{\mathbb{H}^{2}}\left(\rho=r, \theta \in\left(\alpha, \frac{\pi}{2}\right)\right) \\
& =\int_{\alpha}^{\frac{\pi}{2}} \frac{r d \theta}{r \sin (\alpha)} \\
& =\ln \left(\cot \left(\frac{\alpha}{2}\right)\right) .
\end{aligned}
$$

Notice that the above number does not depend on $r$, so the distance from any point of $y=\tan (\alpha) x$ to $\gamma$ is constant.

The distance from the vertical axis to $r_{\alpha}^{-}$is the same above number, since the symmetry with respect to the vertical axis $T(x, y)=(-x, y)$ is a hyperbolic isometry. This shows item (1). 
The proof of item (2) is similar. Parametrize $I_{\alpha, \rho_{1}, \rho_{2}}$ in polar coordinates, we get the curve $\left(\rho \in\left[\rho_{1}, \rho_{2}\right], \theta=\alpha\right)$. So the length of $I_{\alpha, \rho_{1}, \rho_{2}}$ is given by

$$
l_{\mathbb{H}^{2}}\left(I_{\alpha, \rho_{1}, \rho_{2}}\right)=\int_{\rho_{1}}^{\rho_{2}} \frac{d r}{r \sin (\alpha)}=\ln \left(\frac{\rho_{2}}{\rho_{1}}\right) \frac{1}{\sin (\alpha)} .
$$

To get the expression of $l_{\mathbb{H}^{2}}\left(I_{\alpha, \rho_{1}, \rho_{2}}\right)$ in terms of the distance $s(\alpha)$ from $r_{\alpha}^{+}$to the vertical axis just replace in the above formula the equation for $s(\alpha)$ obtained in item (1).

Lemma 4.5. Let $S_{\alpha} \in \mathbb{H}^{2}$ be the cone bounded by $y=\tan (\alpha) x, x>0$, and and the horizontal axis $y=0$. Let $P: S_{\alpha} \longrightarrow\{y=\tan (\alpha) x\}$ be the hyperbolic orthogonal projection onto $y=\tan (\alpha) x$, i.e., the projection along hyperbolic geodesics which are perpendicular to $y=\tan (\alpha) x$. Then $P$ shrinks the hyperbolic length of curves: if $c:[0,1] \longrightarrow S_{\alpha}$ is a smooth curve contained in $S_{\alpha}$, then $l_{\mathbb{H}^{2}}(P(c)) \leq l_{\mathbb{H}^{2}}(c)$.

Proof. Assume without loss of generality that $c$ is a simple curve and that $\rho(c(0)) \leq \rho(c(1))$. It is not difficult to show that there exists a partition $0=t_{0}<t_{1}<. .<t_{k}<t_{k+1}=1$ of $[0,1]$ such that

1. The hyperbolic geodesic $c_{i}=\left[c\left(t_{i}\right), c\left(t_{i+1}\right)\right]$ is contained in $S_{\alpha}$ for every $i=0,1, . ., k$.

2. $\rho\left(c\left(t_{i}\right)\right) \leq \rho\left(c\left(t_{i+1}\right)\right)$ for every $i=0,1, . ., k$.

Since each $c_{i}$ is an arc of circle or a vertical segment, and $\rho\left(c\left(t_{i}\right)\right)<$ $\rho\left(c\left(t_{i+1}\right)\right)$, we get that the restriction of the projection $P$ to $c_{i}$ is an increasing function for every $i=0,1, . ., k$. Moreover,

$$
P\left(c_{i}\right)=I_{\alpha, \rho\left(c\left(t_{i}\right)\right), \rho\left(c\left(t_{i+1}\right)\right)}
$$

for every $i=0,1, \ldots k$. So we can parametrize each geodesic $c_{i}$ by the radius $\rho$. We get monotone parametrizations

$$
c_{i}(\rho)=(\rho \cos (\theta(\rho)), \rho \sin (\theta(\rho)))
$$

for $\rho \in\left[\rho\left(c\left(t_{i}\right)\right), \rho\left(c\left(t_{i+1}\right)\right)\right]$.

Observe that $\theta(\rho) \leq \alpha$ for every $t \in[0,1]$ because $c_{i} \subset S_{\alpha}$ for every $i$. Since $\sin (\theta)$ is increasing in $\theta \in\left[0, \frac{\pi}{2}\right]$, we get that $\frac{1}{\sin (\theta(\rho))} \geq \frac{1}{\sin (\alpha)}$ for every $\rho \in\left[\rho\left(c\left(t_{i}\right)\right), \rho\left(c\left(t_{i+1}\right)\right)\right]$, and every $i=0,1, . . k$. Calculating the hyperbolic length of $c_{i}$ we have

$$
\begin{aligned}
l_{\mathbb{H}^{2}}\left(c_{i}\right) & =\int_{\rho\left(c\left(t_{i}\right)\right)}^{\rho\left(c\left(t_{i+1}\right)\right)} \frac{\sqrt{1+\rho^{2}\left(\theta^{\prime}(\rho)\right)^{2}}}{\rho \sin (\theta(\rho))} d \rho \\
& \geq \int_{\rho\left(c\left(t_{i}\right)\right)}^{\rho\left(c\left(t_{i+1}\right)\right)} \frac{1}{\rho \sin (\alpha)} d \rho \\
& =\ln \frac{\rho\left(c\left(t_{i+1}\right)\right)}{\rho\left(c\left(t_{i}\right)\right)} \frac{1}{\sin (\alpha)}=l_{\mathbb{H}^{2}}\left(I_{\alpha, \rho\left(c\left(t_{i}\right)\right), \rho\left(c\left(t_{i+1}\right)\right)}\right) .
\end{aligned}
$$


So we get that $l_{\mathbb{H}^{2}}\left(c_{i}\right) \geq l_{\mathbb{H}^{2}}\left(P\left(c_{i}\right)\right)$ for every $i=0,1, . ., k$. Since the sum of the lengths of the $c_{i}$ 's at most the length of $c$, and the union of the projections by $P$ of the $c_{i}$ 's is contained in the projection by $P$ of $c$, we conclude that $l_{\mathbb{H}^{2}}(c) \geq l_{\mathbb{H}^{2}}(P(c))$ as we wished to show.

\section{Proof of Theorem 4.2}

Let $c:[a, b] \longrightarrow \mathbb{H}^{2}$ be an $A, B$ quasi-geodesic, which we suppose to be the union of a finite number of smooth curves. Let $[c(a), c(b)]=\beta$ be the hyperbolic geodesic joining $c(a)$ to $c(b)$. We shall show that there exists $D>0, D=D(A, B)$, such that $c([a, b])$ is contained in the tubular neighborhood $V_{D}(\beta)=\left\{p \in \mathbb{H}^{2}, d_{\mathbb{H}^{2}}(p, \beta) \leq D\right\}$ of $\beta$ with radius $D$.

Since the group of isometries of $\mathbb{H}^{2}$, the orientation preserving Moebius transformations, act transitively in $\mathbb{H}^{2}$, we can suppose that $\beta$ is a vertical segment parametrized as $\gamma(t)=(0, t), t \in\left[t_{1}, t_{2}\right]$. In this way, $c$ is a quasigeodesic joining two points in the vertical axis, $c(a)=\gamma\left(t_{1}\right), c(b)=\gamma\left(t_{2}\right)$. Let $\rho(c(t))$ be the radial polar coordinate of $c(t)$.

Let us suppose that for $r>0$, the curve $c$ has some points outside $V_{r}(\gamma)$. Let $C_{\alpha_{r}}$ be the corresponding cone. Let $S_{\alpha_{r}}$ be the complement of $C_{\alpha_{r}}$ in the first quadrant. In this way, we can separate the set

$$
E_{r}=\left\{t \in[a, b], c(t) \cap S_{\alpha_{r}} \neq \emptyset\right\}
$$

into two subsets: $[a, b]=U_{1}^{r} \cup U_{2}^{r}$, where $U_{1}^{r}$ is the set of connected components $[s, t]$ of $E$ where $\frac{\rho(c(t))}{\rho(c(s))} \leq e$, and $U_{2}^{r}$ is the set of connected components of $E$ where $\frac{\rho(c(t))}{\rho(c(s))}>e$.

Observe that if $[s, t] \in U_{1}^{r}$, we have that the length of $I_{\alpha, \rho(c(s)), \rho(c(t))}$ is bounded above by $\csc \left(\alpha_{r}\right)$. And since $c$ is a $A, B$-quasi geodesic, we have that

$$
l_{\mathbb{H}^{2}}(c([s, t])) \leq A d_{\mathbb{H}^{2}}(c(s), c(t))+B \leq A \csc \left(\alpha_{r}\right)+B .
$$

So the length of each component in $U_{1}^{r}$ is bounded by a universal constant $\kappa_{r}=A \csc \left(\alpha_{r}\right)+B$.

Claim: There exists $r_{0}=r_{0}(A, B)$ such that if $[t, s] \in U_{2}^{r}$, then $r \leq r_{0}$.

In fact, assume that $c[s, t])$ is contained in $U_{2}^{r}$. Let $\sigma \in[s, t]$ such that

$$
d_{\mathbb{H}^{2}}\left(c([s, t]),\left\{y=\tan \left(\alpha_{r}\right) x\right\}\right)=d_{\mathbb{H}^{2}}\left(c(\sigma),\left\{y=\tan \left(\alpha_{r}\right) x\right\}\right)=\nu .
$$


Assume that $\rho(s) \leq \rho(\sigma) \leq \rho(t)$. The reasoning applied in this case also holds in the general case. Since $c$ is an $A, B$-quasi geodesic we have

$$
\begin{aligned}
l_{\mathbb{H}^{2}}(c([s, t])) & =l_{\mathbb{H}^{2}}(c(s), c(\sigma))+l_{\mathbb{H}^{2}}(c(\sigma), c(t)) \\
& \leq A\left(d_{\mathbb{H}^{2}}(c(s), c(\sigma))+d_{\mathbb{H}^{2}}(c(\sigma), c(t))\right)+2 B \\
& \leq A\left(2 r+2 \nu+\ln \left(\frac{\rho(c(t))}{\rho(c(\sigma))}\right)+\ln \left(\frac{\rho(c(\sigma))}{\rho(c(t))}\right)+2 B\right. \\
& =A\left(2(2 r+\nu)+\ln \left(\frac{\rho(c(t))}{\rho(c(s))}\right)\right)+2 B .
\end{aligned}
$$

Notice that

$$
\begin{aligned}
\nu \leq l_{\mathbb{H}^{2}}(c([s, t])) & \leq A d_{\mathbb{H}^{2}}(c(s), c(t))+B \\
& \leq A \ln \left(\frac{\rho(c(t))}{\rho(c(s))}\right)+B .
\end{aligned}
$$

Combining with the previous estimate, we get

$$
l_{\mathbb{H}^{2}}(c([s, t])) \leq A(2 A+1) \ln \left(\frac{\rho(c(t))}{\rho(c(s))}\right)+B(2+A)+4 A r .
$$

Since the orthogonal projection $I_{\alpha_{r}, \rho(c(s)), \rho(c(t))}$ of $c([s, t])$ onto $\left\{y=\tan \left(\alpha_{r}\right) x\right\}$ has less hyperbolic length than $c([s, t])$ (Lemma 4.5), we have

$$
\begin{aligned}
\ln \left(\frac{\rho(c(t))}{\rho(c(s))}\right) \cosh (r) & =l_{\mathbb{H}^{2}}\left(I_{\alpha_{r}, \rho(c(s)), \rho(c(t))}\right) \\
& \leq A(2 A+1) \ln \left(\frac{\rho(c(t))}{\rho(c(s))}\right)+B(2+A)+4 A r .
\end{aligned}
$$

Dividing this inequality by $Q=\ln \left(\frac{\rho(c(t))}{\rho(c(s))}\right)>1$, we obtain

$$
\cosh (r) \leq A(2 A+1)+B(2+A)+4 A r .
$$

Since $\cosh (r)$ is an exponential function of $r$, there exists $r_{0}>0$ such that the inequality holds if and only if $r \leq r_{0}$. This finishes the proof of the Claim.

From the Claim we get that there exists $r_{0}$ such that if the set $U_{2}^{r}$ is nonempty for some $r$, then $r \leq r_{0}$. From the the definition of $U_{1}^{r}$, it is clear that there is a number $R_{0}>0$ such that either there exists $r$ with $U_{2}^{r}=\neq \emptyset$, or the length of $c([a, b])$ is bounded above by $R_{0}$. In the latter case the statement of the Lemma follows. In the former case, it follows from the Claim and the fact that the length of a connected component in $U_{1}^{r}$ is at most $\kappa_{r_{0}}$. This finishes the proof Theorem 4.2 in the case where $[a, b]$ is finite. 
If $c:(-\infty,+\infty) \longrightarrow \mathbb{H}^{2}$ is a $A, B$-quasi geodesic, then we consider a sequence $\left[a_{n}, b_{n}\right]$ of intervals where $a_{n} \rightarrow-\infty, b_{n} \rightarrow+\infty$, and apply the previous result to each quasi geodesic $c\left(\left[a_{n}, b_{n}\right]\right)$. We get a sequence of geodesics $\gamma_{n}:\left[s_{n}, t_{n}\right] \longrightarrow \mathbb{H}^{2}$ which are contained in a tubular neighborhood of radius $D$ of $c\left(\left[a_{n}, b_{n}\right]\right)$. It is clear that there exists a convergent subsequence of the $\gamma_{n}$ whose limit is a geodesic $\gamma_{\infty}$, that is in the $D$-tubular neighborhood of $c$, as we wished to show. This finishes the proof of Theorem 4.2.

Morse's result has strong consequences on the global geometry of geodesics. For instance, we get that $(\tilde{M}, \tilde{g})$ is a quasi-convex space.

Definition 4.2. A complete Riemannian manifold $(N, h)$ is $K, C$-quasiconvex if given $a_{1}, a_{2}, b_{1}, b_{2}$ in $N$, then any pair of geodesics $\left[a_{1}, a_{2}\right]$ joining $a_{1}, a_{2}$, and $\left[b_{1}, b_{2}\right]$ joining $b_{1}, b_{2}$, satisfies

$$
d\left(\left[a_{1}, a_{2}\right],\left[b_{1}, b_{2}\right]\right) \leq K \sup \left\{d\left(a_{1}, b_{1}\right), d\left(a_{2}, b_{2}\right)\right\}+C,
$$

where $d\left(\left[a_{1}, a_{2}\right],\left[b_{1}, b_{2}\right]\right)$ is the Haussdorff distance from one geodesic to the other.

Corollary 4.1. Let $(M, g)$ be a compact surface without conjugate points and genus greater than one. Then $(\tilde{M}, \tilde{g})$ is a quasi-convex space.

Proof. This follows essentially from Proposition 4.2, which asserts that every geodesic in $(\tilde{M}, \tilde{g})$ is globally minimizing; combined with Theorem 4.2 and the triangular inequality.

The proof of Morse's theorem (Theorem 4.2) can be extended to any compact manifold admitting a metric with negative curvature. Eberlein and O'Neil [35] introduced visibility manifolds and showed the shadowing of quasi-geodesics by geodesics. Such manifolds are in fact manifolds where geodesic rays diverge and whose fundamental groups are Gromov hyperbolic, a subject that will be treated later on (Chapters 6, 7) in this survey.

Corollary 4.2. Let $(M, g)$ be a compact surface of genus greater than one without conjugate points. Let $\left(M, g_{1}\right)$ be any other metric in $M$ without conjugate points. Then there exists $r>0$ depending on $g, g_{1}$, such that for every $\tilde{g}_{1}$-geodesic $\gamma_{1} \in\left(\tilde{M}, \tilde{g}_{1}\right)$ there exists a $\tilde{g}$-geodesic $\gamma \in(\tilde{M}, \tilde{g})$ such that

$$
d\left(\gamma, \gamma_{1}\right) \leq r,
$$

where the above distance is the Hausdorff distance.

Proof. Another straightforward consequence of Theorem 4.2. Let $\left(M, g_{1}\right)$ be any metric without conjugate points in the surface $M$. Then the shadowing of the $\tilde{g}_{1}$-geodesics by hyperbolic geodesics, and the shadowing of the $\tilde{g}$-geodesics by hyperbolic geodesics, implies by the triangular inequality that $\tilde{g}$-geodesics shadow $\tilde{g}$-geodesics. 
The quasi-convexity of $(\tilde{M}, \tilde{g})$ and the shadowing of geodesics are remarkable consequences of the topological classification of compact surfaces. Usually, any information about the global behavior of geodesics is obtained from some information about the local geometry (curvature) of the surface. To explore the relationships between global properties of geodesics and the topology of manifolds is one of the main goals of the survey.

\section{Divergence of geodesic rays and continuity of $H_{\theta}(0)$ with respect to $\theta$}

We continue our survey of results about surfaces without conjugate points with the analysis of two very natural questions of the theory of manifolds without conjugate points:

1. Do geodesic rays diverge in $\tilde{M}$ ?

2. Do horospheres $H_{\theta}$ depend continuously on $\theta$ ?

Definition 4.3. We say that two geodesic rays $\gamma(t), \beta(t)$ in $\tilde{M}$, with $\gamma(0)=$ $\beta(0)$ diverge if $\lim _{t \rightarrow+\infty} d(\gamma(t), \beta(t))=+\infty$. We say that geodesic rays diverge uniformly in $\tilde{M}$ if given $\epsilon>0, D>0$, there exist $T=T(\epsilon, D)>0$ such that if $\gamma, \beta$ are two geodesics with $\gamma(0)=\beta(0)$ which form an angle greater than $\epsilon$ at $t=0$, we have that $d(\gamma(t), \beta(t)) \geq D$ for every $t \geq T$.

Geodesic rays diverge in manifolds with nonpositive curvature due to the convexity of the norm of Jacobi fields and the definition of the exponential map (Lemma 1.2).

The continuity of $\theta \rightarrow H_{\theta}(0)$ in the compact open topology is defined as follows: let $\theta_{n}$ converge to $\theta$, then $H_{\theta_{n}}(0)$ converges to $H_{\theta}(0)$ uniformly on compact subsets of $\tilde{M}$. Although by Lemma 4.2 it is clear that $H_{\theta}(t)$ depends continuously on $t \in R$, it is not known whether $H_{\theta}(0)$ depends continuously on $\theta$. The continuity of $\theta \rightarrow H_{\theta}$ is equivalent to the continuity in the $C^{1}$ topology of the map $\theta \rightarrow b^{\theta}$ uniformly on compact subsets of $\tilde{M}$.

Horospheres $H_{\theta}(0)$ depend continuously on $\theta$ in manifolds with nonpositive curvature and sectional curvatures bounded from below. The continuity of horospheres and the divergence of geodesic rays are closely related through the notion of asymptoticity of geodesics in $\tilde{M}$.

\subsection{Asymptoticity and quasi convexity}

Definition 4.4. A geodesic $\beta$ is asymptotic to a geodesic $\gamma$ in $\tilde{M}$ if there exists a constant $C>0$ such that $d(\beta(t), \gamma(t)) \leq C$ for every $t \geq 0$. 
We shall denote by Busemann asymptotes of $\gamma_{\theta}$ the orbits of the flow $\psi_{t}^{\theta}$.

Lemma 4.6. Let $(M, g)$ be a compact manifold without conjugate points such that $(M, g)$ is quasi-convex (Def. 4.2). Then Busemann asymptotes of $\gamma_{\theta}$ are asymptotic to $\gamma_{\theta}$.

Proof. This is a consequence of the quasi-convexity of $(M, g)$ and the definition of Busemann asymptotes. Recall that each Busemann asymptote $\beta$ of $\gamma_{\theta}$ is defined as a limit as $T \rightarrow+\infty$ of geodesics of the form $\beta_{T}=\left[\beta(0), \gamma_{\theta}(T)\right]$, where the notation $[p, q]$ means the geodesic joining $p$ to $q$ (we already know that $[p, q]$ it is unique). Let $\gamma_{\theta}\left(t_{0}\right)$ be the closest point of $\gamma_{\theta}$ to $\beta(0)$. By quasi-convexity we have constants $A, B$ such that

$$
d_{H}\left(\gamma_{\theta}\left(\left[t_{0}, T\right]\right), \beta_{T}\right) \leq A d\left(\gamma_{\theta}\left(t_{0}\right), \beta(0)\right)+B,
$$

where $d_{H}$ is the Hausdorff distance. Clearly, this estimate extends to the limit if $T \rightarrow+\infty$, so we get

$$
d_{H}\left(\gamma_{\theta}\left(\left[t_{0}, \infty\right)\right), \beta([0, \infty)) \leq A d\left(\gamma_{\theta}\left(t_{0}\right), \beta(0)\right)+B\right.
$$

thus proving that $\beta$ is asymptotic to $\gamma_{\theta}$.

Corollary 4.3. If $(M, g)$ is a compact surface without conjugate points, then Busemann asymptotes of $\gamma_{\theta}$ are asymptotic to $\gamma_{\theta}$ for every $\theta \in T_{1} \tilde{M}$.

This is just a combination of the quasi-convexity of compact surfaces without conjugate points (Corollary 4.1 and the previous lemma).

In higher dimensions, Busemann asymptotes of $\gamma_{\theta}$ might not be asymptotic to $\gamma_{\theta}$. Lemma 4.6 can be applied to manifolds with nonpositive curvature and without focal points.

Definition 4.5. A complete Riemannian manifold $(M, g)$ without conjugate points has no focal points if for every $(p, v) \in T M, v \neq 0$, the restriction of the exponential map $\exp _{p}$ to the subspace of $T_{p} M$ that is perpendicular to $v$ is nonsingular.

There are many equivalent ways to define manifolds without focal points, we summarize some of them with the help of the tools developed in Chapter 3 .

Lemma 4.7. A complete manifold $(M, g)$ without conjugate points has no focal points if and only if:

1. The second fundamental form of every sphere in $(\tilde{M}, \tilde{g})$ is semidefinite. 
2. The norm of the stable Jacobi fields defined in Chapter 3 is always non-increasing, and the norm of unstable Jacobi fields in always nondecreasing.

3. The matrix solutions $U_{\theta}^{s}(t)$ of the Riccati equation (eq. 3.1) are negative semi-definite for every $\theta \in T_{1} M, t \in \mathbb{R}$; and the matrix solutions $U_{\theta}^{u}(t)$ are positive semi-definite for every $\theta \in T_{1} M, t \in \mathbb{R}$.

The proof of the above lemma is a good exercise and we leave it to the reader. It is easy to check, using Lemma 4.7, that manifolds with nonpositive curvature have no focal points. Moreover, from Lemma 4.7 we can easily deduce that the universal covering of a complete manifold without focal points is 1,0-quasi convex. So Busemann asymptotes are true asymptotes in this case. The theory of manifolds without focal points is an important, well understood part of the theory of manifolds without conjugate points. They were studied by Eberlein, Pesin, Eschenburg and many others, and most of the results known for manifolds with nonpositive curvature generalize to such manifolds.

In the case of manifolds without conjugate points, Croke-Schröeder [26] showed that two different bi-asymptotic lifts $\gamma_{\theta}, \gamma_{\sigma}$ of a closed geodesic $\gamma \in M$ have the property that the corresponding Busemann functions differ by a constant, provided that $(M, g)$ is analytic. This statement implies in particular that $\gamma_{\theta}$ and $\gamma_{\sigma}$ are Busemann asymptotes of each other. In fact, using the tools developed in [26] it is not difficult to show that

Lemma 4.8. Let $(M, g)$ be compact without conjugate points. Let $\gamma_{\theta}, \gamma_{\sigma}$ be two different, bi-asymptotic lifts in $\tilde{M}$ of a closed geodesic $\gamma \in M$. Then they are Busemann asymptotes of each other.

We shall prove Lemma 4.8 in Chapter 6, using elementary properties of the fundamental group of manifolds without conjugate points.

\subsection{Divergence of geodesic rays in surfaces without conjugate points}

In the previous section we analyzed the problem of knowing whether a Busemann asymptote of a given geodesic in $\tilde{M}$ is a true asymptote of the geodesic. In the present section we look at a sort of converse of this problem: given a geodesic that is asymptotic to a given one, is it Busemann asymptotic? The answer to this question is closely related with the divergence of geodesic rays.

More generally, the issue of the divergence of geodesic rays comes in when we ask the following question: given a geodesic $\gamma \subset \tilde{M}$, and a point $p \in \tilde{M}$, is there a unique geodesic asymptotic to $\gamma$ starting at $p$ ? In the 
case of quasi convex manifolds, Lemma 4.6 shows that such an asymptotic geodesic always exists. But the quasi convexity assumption does not grant the uniqueness of the asymptote. If we had two geodesics asymptotic to $\gamma$ starting at $p$, then we would have two non diverging geodesic rays. In particular, if the manifold has nonpositive curvature there is a unique geodesic asymptotic to $\gamma \subset \tilde{M}$ passing through $p \in \tilde{M}$. It is not difficult to show that the above assertion also holds for manifolds without focal points.

In compact surfaces without conjugate points, the problem of the divergence of geodesic rays was solved by Hopf and Green [44]. Hopf's theorem (Theorem 4.1) says that if the surface is a torus then the metric is flat. Therefore, the universal covering is just Euclidean space and clearly geodesic rays (straight lines) diverge. For surfaces of higher genus, the divergence of geodesic rays is also true by the work of Green.

Proposition 4.1. Let $(M, g)$ be a compact surface without conjugate points. Then geodesic rays diverge uniformly in $\tilde{M}$.

Proof. The argument of the proof relies strongly in two dimensional topology, it does not extend to higher dimensions. Suppose by contradiction, that we have $p \in \tilde{M}$, and two geodesics $\gamma_{(p, v)}, \gamma_{(p, w)}$, such that

$$
d\left(\gamma_{(p, v)}(t), \gamma_{(p, w)}(t)\right) \leq C
$$

for every $t \geq 0$. Let us assume that the pair $(v, w)$ has the canonical orientation of $\tilde{M}$.

The set $\left\{\gamma_{(p, v)}(t), t \geq 0\right\} \cup\left\{\gamma_{(p, w)}(t), t \geq 0\right\}$ bounds a geodesic cone $S \in \tilde{M}$ with the following properties:

1. There exists a smooth curve $c:[0, a] \longrightarrow T_{p} \tilde{M}$ of normal vectors $c(s)$ such that $c(0)=v, c(a)=w$, and $\left\|c^{\prime}(s)\right\|=1$ for every $s \in[0, a]$.

2. The cone $S$ is given by

$$
S=\left\{\exp _{p}(t c(s)), t \geq 0, s \in[0, a]\right\} .
$$

The set $S_{t}=\left\{\exp _{p}(t c(s)), s \in[0, a]\right\}$ is an arc of the sphere $S_{t}(p)$ of radius $t$ centered at $p$. Recall that $S_{t}(p)$ has bounded geometry if $t \geq 2$ say, namely, the curvatures of large spheres are bounded by a uniform constant (Lemma 4.2). So the assumption $d\left(\gamma_{(p, v)}(t), \gamma_{(p, w)}(t)\right) \leq C$ for every $t \geq 0$ implies that there exists a constant $L_{0}>0$ such that the length of the arcs $S_{t}$ is bounded above by $L_{0}$ for every $t \geq 0$. The length of $S_{t}$ is given by

$$
l_{\tilde{g}}\left(S_{t}\right)=\int_{0}^{a}\left\|D_{t c(s)} \exp _{p}\left(t c^{\prime}(s)\right)\right\| d s=\int_{0}^{a}\left\|J_{s}(t)\right\| d s,
$$

where $J_{s}(t)=D_{t c(s)} \exp _{p}\left(t c^{\prime}(s)\right)$ is a Jacobi field according to Lemma 1.2. This Jacobi fields vanish at $t=0$, and $J_{s}^{\prime}(0)=c^{\prime}(s)$ for every $s \in[0, a]$. So $\left\|J_{s}^{\prime}(0)\right\|=1$ for every $s$. 
The fact that $l_{\tilde{g}}\left(S_{t}\right) \leq L_{0}$ for every $t \geq 0$ implies that

$$
\int_{0}^{a}\left\|J_{s}(t)\right\| d s \leq L_{0}
$$

for every $t \geq 0$. Applying the mean value theorem for integrals, given $t>0$, there exists $s_{t} \in[0, a]$ such that

$$
\left\|J_{s_{t}}(t)\right\| \leq a L_{0} .
$$

On the other hand, Proposition 3.1 in the case of surfaces implies that given $r>0$ there exists $T=T(r)>0$ such that $\left\|J_{s}(t)\right\| \geq r$ for every $t \geq T$. Indeed, Proposition 3.1 says that Jacobi fields which vanish at a point diverge uniformly with respect to the norm of its derivative. This contradicts the assumption of the non divergence of rays, so we get Proposition 4.1.

Remark that the argument in the proof of Proposition 4.1 fails in higher dimensions essentially because of the (possibly) non uniform divergence of vanishing Jacobi fields. In fact, the divergence of vanishing Jacobi fields might depend on the geodesic where it is defined, and not only on the norm of its initial derivative. This is the best result known about divergence of vanishing Jacobi fields, it was proved by Eberlein [32] as we already observed in Chapter 3.

Corollary 4.4. Let $(M, g)$ be a compact surface without conjugate points. Given a geodesic $\gamma \subset \tilde{M}$, and $p \in \tilde{M}$, there is a unique geodesic asymptotic to $\gamma$ containing $p$. This geodesic is the Busemann asymptote of $\gamma$ passing through $p$. The relation between geodesics $\gamma \mathbf{R} \beta$ if and only if " $\gamma$ is a Busemann asymptote of $\beta$ " is an equivalence relation.

To conclude the subsection, we would like to discuss the last part of the statement of Corollary 4.4 in general dimensions. Assuming that the manifold has nonpositive curvature or even no focal points the Corollary remains true. This is because in such manifolds we have both divergence of geodesic rays and quasi-convexity of $(\tilde{M}, \tilde{g})$. We could expect that the above relation is an equivalence relation in the category of manifolds without conjugate points. Observe that if we drop the word "Busemann" from the definition of the relation, it becomes obviously an equivalence relation.

The main difficulty to prove the equivalency of the relation of being Busemann asymptote is precisely the reflexive property. As far as we know, this is an open problem of the theory of manifolds without conjugate points. We continue to deal with the divergence of rays in higher dimensions in the next subsections. 


\subsection{Divergence of geodesic rays in higher dimensions}

The previous discussion can be generalized in higher dimensions in the following statement.

Lemma 4.9. Let $(M, g)$ be a compact manifold without conjugate points. Suppose that geodesic rays diverge in $\tilde{M}$. Then any geodesic asymptotic to $\gamma \subset \tilde{M}$ is a Busemann asymptote of $\gamma$.

The (possibly) non uniform divergence of vanishing Jacobi fields motivated Eberlein to define an ad hoc condition on Jacobi fields, the so-called bounded asymptote condition.

Definition 4.6. The manifold $(M, g)$ without conjugate points satisfies the bounded asymptote condition if there exists $C>0$ such that every stable Jacobi field $J(t)$ satisfies $\|J(t)\| \leq C\|J(0)\|$ for every $t \geq 0$.

Of course, the convexity of the metric (nonpositive curvature and no focal points) imply the bounded asymptote condition. However, it is very hard to prove it if we don't know something about the local geometry of the manifold. Moreover, the bounded asymptote condition implies that $(\tilde{M}, \tilde{g})$ is $C, 0$-quasi-convex, according to Definition 4.2 .

Manifolds with bounded asymptote were studied for instance by Eschenburg [37], Croke-Kleiner [25], and Knieper [60].

Lemma 4.10. [60] Let $(M, g)$ be a compact manifold without conjugate points and bounded asymptote. Then vanishing Jacobi fields diverge at least linearly. Namely, there exists $C>0$ such that given a nontrivial Jacobi field $J(t)$ with $J(t)=0$, we have that

$$
\|J(t)\| \geq C\left\|J^{\prime}(0)\right\| t
$$

for every $t \geq 0$.

With this result, we can show that geodesic rays diverge with the same order of magnitude.

Corollary 4.5. [89] Let $(M, g)$ be a compact manifold without conjugate points and bounded asymptote. Then geodesic rays diverge at least linearly. Namely, there exists $C>0$ such that given two geodesic rays $\gamma_{(p, v)}, \gamma_{(p, w)}$, we have that

$$
d\left(\gamma_{(p, v)}(t), \gamma_{(p, w)}(t)\right) \geq C \measuredangle(v, w) t,
$$

for every $t \geq 0$, where $\measuredangle(v, w)$ is the angle formed by $v, w$. 


\subsection{The continuity of horospheres}

In this subsection we analyze the link between divergence of geodesic rays and the continuity of horospheres. The following elementary remark summarizes the main idea behind all the results that will be presented in the subsection: let $(M, g)$ be compact without conjugate points. The map $\theta \rightarrow b^{\theta}$ is continuous in the compact open topology of $\tilde{M}$ (according to the definition given at the beginning of the section) if and only if then the map $\theta \rightarrow H_{\theta}(0)$ is continuous. Indeed, if the Busemann functions $b^{\theta}$ depend continuously on $\theta$ in the $C^{1}$ topology, their level sets $H_{\theta}(t)$ will depend continuously on $\theta$ as well.

So the continuity of $\theta \rightarrow H_{\theta}(0)$ is equivalent to the continuous dependence of Busemann asymptotes upon their initial data. This is not easy to show in a manifold without conjugate points and no further hypothesis. Pesin [75] introduced a category of manifolds without conjugate points that is inspired by this remark.

Definition 4.7. Let $(M, g)$ has no conjugate points. $(\tilde{M}, \tilde{g})$ satisfies the asymptoticity condition if every geodesic $\gamma$ in $\tilde{M}$ has the following property:

Given any sequence of points $p_{n} \in \tilde{M}$ converging to $x \in \tilde{M}$, and any sequence of points $q_{n} \in \tilde{M}$ such that

1. $\lim _{n \rightarrow+\infty} d\left(q_{n}, \gamma(0)\right)=+\infty$,

2. There exists a constant $D>0$ such that $d\left(q_{n}, \gamma\right) \leq D$ for every $n$,

then the sequence of geodesic segments $\left[p_{n}, q_{n}\right]$ converges to a geodesic $\gamma_{x}$ -depending only on $x$ - that is asymptotic to $\gamma$.

The asymptoticity condition is a little technical, but is weaker than the bounded asymptote condition because it is an assumption made on the geodesics and not on Jacobi fields. The example of Ballmann-Brin-Burns [6] of a compact surface without conjugate points does not satisfies the bounded asymptote condition. Nevertheless, geodesic rays diverge and horospheres depend continuously in the compact open topology.

Notice that the geodesic $\gamma_{x}$ in the above definition only depends on the point $x$ and is independent of the sequences $q_{n}, q_{n}$. So the asymptotic geodesic $\gamma_{x}$ is the unique geodesic asymptotic to $\gamma$ through $x$.

Pesin in [75], showed that under the asymptoticity axiom, $H_{\theta}(0)$ depend continuously on $\theta \in \tilde{M}$ according to the notion of continuity given at the beginning of the section. Actually, Pesin's axiom of asymptoticity implies the continuity of horospheres almost by its definition. We leave the details of this proof to the reader. 
Unfortunately, the axiom of asymptoticity is very hard to check it in a manifold without conjugate points and no further hypothesis on the geometry. Surfaces without conjugate points satisfy the asymptoticity condition by Corollary 4.4. If the curvature of the manifold is nonpositive, the axiom of asymptoticity holds as well since the argument in the proof of Corollary 4.4 can be extended to such manifolds: it is based on quasi-convexity of $(\tilde{M}, \tilde{g})$ and divergence of geodesic rays, both hold in nonpositive curvature.

Lemma 4.11. Let $(M, g)$ be a compact manifold without conjugate points. Suppose that $(\tilde{M}, \tilde{g})$ is quasi-convex and that geodesic rays diverge in $(M, g)$. Then the map $\theta \rightarrow H_{\theta}(0)$ is continuous.

Finally, the following somehow surprising result gives some light about the relevance of the asymptoticity condition in the continuity of horospheres.

Theorem 4.3. [91] Let $(M, g)$ be a compact manifold without conjugate points. If $\theta \rightarrow H_{\theta}(0)$ depends continuously on $\theta \in T_{1} \tilde{M}$, then geodesic rays diverge uniformly in $\tilde{M}$.

This theorem is interesting because it has no hypothesis on the geometry of the manifold, nor on the geodesics or Jacobi fields. It might suggest that the divergence of geodesic rays should hold without the need of quasiconvexity or asymptoticity conditions. As a consequence of this theorem we get that Pesin's asymptoticity condition implies the divergence of geodesic rays. It would be interesting to show that divergence of geodesic rays and continuity of horospheres are equivalent.

\section{Summary of results about the geodesic flow of compact surfaces without conjugate points}

We would like to conclude our discussion about compact surfaces without conjugate points with a summary of the most important results about the geodesic flow. The topological dynamics of the geodesic flow, as well as important results in ergodic theory, foliation theory and rigidity, are well known in the case of surfaces. Many of such results are open problems in higher dimensions.

1. The geodesic flow of compact surfaces without conjugate points and genus greater than one is $C^{0}$ semi-conjugate to the geodesic flow of a hyperbolic surface [42]. 
2. The collection of central stable (unstable) forms a foliation of $T_{1} M$. If the genus of the surface is greater than one this foliation is $C^{0}$ conjugate to a hyperbolic central foliation by a (vertical) fiber preserving conjugacy. Moreover, the central foliations are the only codimension one, continuous foliations which are invariant by the geodesic flow [13].

3. Every compact surface whose geodesic flow preserves a codimension one, $C^{1}$ foliation has no conjugate points, and if the foliation is $C^{3}$ then the curvature of the surface is a nonpositive constant [12].

4. The topological entropy of the geodesic flow of a compact surface of genus greater than one is nonzero and there is always a hyperbolic closed geodesic [48].

5. In a compact surface without conjugate points and genus greater than one, if the Green subbundles are continuous the metric entropy with respect to the Lebesgue measure is nonzero [60].

6. If the geodesic flow is Anosov, the foliations $\mathcal{F}^{s}$ and $\mathcal{F}^{u}$ are $C^{1}$, and the flow is ergodic (Hopf [52]).

7. Moreover, if the geodesic flow is Anosov then the stable and unstable foliations are minimal (Hedlund [50]), and in the case of constant negative curvature the so-called horocycle flows are uniquely ergodic (Furstenberg [41]).

For the definitions and the basic theory of measure preserving systems (ergodicity, entropy, etc) we refer to [48]. Observe that all the above properties are well known for Anosov geodesic flows in compact manifolds. It is indeed surprising that two dimensional topology combined with the general structure of Jacobi fields of compact manifolds without conjugate points gives such a rich topological structure for the geodesic flow in compact surfaces with no conjugate points.

We won't discuss these statements in the present survey, however it is worthwhile to mention such properties to get a more complete panorama. Notice that item (2) says that the central foliations in compact surfaces without conjugate points behave like Anosov central foliations, they are the only codimension one continuous invariant foliations. Item (3) generalizes a famous theorem due to E. Ghys [43] involving Anosov geodesic flows of compact surfaces. Items (4) and (5) suggests that the geodesic flow of compact surfaces with higher genus and nonpositive curvature should be ergodic. However, this problem is very hard and remains open so far. 


\section{Higher dimensional manifolds without con- jugate points: Convexity and flats in the universal covering}

Recall that a continuous function $f: \mathbb{R} \longrightarrow \mathbb{R}$ is convex if $f((1-t) x+t y) \leq$ $(1-t) f(x)+t f(y)$ for every $t \in[0,1]$ and $x, y \in \mathbb{R}$. When $f$ is $C^{2}$, it is convex if and only if $f^{\prime \prime}(x) \geq 0$ for every $x$. Convex functions on intervals are defined in an analogous way.

We say that a continuous function $f:(M, g) \longrightarrow \mathbb{R}$ is convex if $f \circ \gamma(t)$ is convex for every geodesic $\gamma$. The existence of convex functions in a Riemannian manifold is quite exceptional, and manifolds with non positive curvature have plenty of them. Let us list some of the convex functions of nonpositive curvature geometry, we follow [34].

Lemma 4.12. Let $(M, g)$ be a complete manifold with nonpositive curvature. Then

1. Let $\gamma$ be either a geodesic of $(M, g)$ or a geodesic of $(\tilde{M}, \tilde{g})$. Let $J(t)$ be a Jacobi field on $\gamma$. Then the norm of $J(t)$ is a convex function.

2. If $C \subset \tilde{M}$ is a convex subset, the function $d_{C}: \tilde{M} \longrightarrow \mathbb{R}$ given by $d_{C}(p)=d(p, C)=\inf \{d(p, q), q \in C\}$ is convex, where $d$ is the distance with respect to $\tilde{g}$.

3. The distance to a given point in $\tilde{M}$ is a convex function. In particular, spheres in $\tilde{M}$ are convex submanifolds (i.e., their second fundamental forms are definite) and balls are convex subsets.

4. Busemann functions $b^{\theta}$ are convex, and subsets of the form $b^{\theta} \leq T$ are convex subsets of $\tilde{M}$ for every $T \in \mathbb{R}$.

5. If $F$ is an isometry of $(\tilde{M}, \tilde{g})$, then the displacement of $F, d_{F}: \tilde{M} \longrightarrow$ $\mathbb{R}$ given by $d_{F}(x)=d(x, F(x))$ is a convex function.

The convexity of distance functions has strong consequences in the geometry of nonpositive curvature. We list some of the main properties of the global geometry of $\tilde{M}$ which follow from convexity. A good reference for this subject is [8].

Lemma 4.13. 1. The flat strip theorem: any two bi-asymptotic geodesics in $\tilde{M}$ bound a flat strip that is a convex set. The set of bi-asymptotic geodesics to a given one in $\tilde{M}$ is a convex set foliated by bi-asymptotic geodesics.

2. There exists a set of bi-asymptotic geodesics to $\gamma_{(p, v)}$ in $\tilde{M}$ if and only if $H_{(p, v)}(t) \cap H_{(p,-v)}(-t)$ contains a convex, nontrivial set for every 
$t \in \mathbb{R}$. Moreover, $H_{(p, v)}(t) \cap H_{(p,-v)}(-t)$ is precisely the intersection of the geodesics which are bi-asymptotic to $\gamma_{(p, v)}$ and $H_{(p, v)}(t)$ for every $t \in \mathbb{R}$.

The flat strip theorem (item (1) above) is one of the fundamental tools in the study of global geometry in nonpositive curvature. We could ask if convexity can exist in manifolds whose curvature has variable sign. The answer is affirmative, there exist manifolds without focal points (Definition 4.5) where the spheres in the universal covering are smooth convex sets and have some regions with positive curvature. Many of the results stated in Lemmas 4.12 and 4.13 hold for manifolds without focal points. In particular, the flat strip theorem holds for such manifolds (see [75] for instance). However, there are deep results of the theory of nonpositive curvature manifolds like the structure theorem of higher rank manifolds [5], [7] which are not known for manifolds without focal points. 


\section{Chapter 5}

\section{Expansive geodesic flows in manifolds without conjugate points: an example of the application of $C^{0}$ methods to study dynamics and global geometry}

An interesting application of $C^{0}$ methods, namely, the study of geodesics without any a priori information about Jacobi fields, in higher dimensions, is the theory of expansive geodesic flows in manifolds without conjugate points.

As we saw in Chapters 2 and 3, Anosov geodesic flows of compact manifolds are examples of expansive geodesic flows without conjugate points (recall that the definition of an expansive flow is Definition 2.12). However, it is not hard to get examples of geodesic flows which are expansive and non Anosov. The expansiveness hypothesis is of purely topological nature, the differential of the geodesic flow (and hence Jacobi fields) do not appear in the definition. Expansive flows are natural generalizations of Anosov flows, and it is natural to ask whether the features of global geometry of manifolds with Anosov geodesic flows can be generalized to manifolds with expansive geodesic flows. This will be the main subject of the chapter. 


\section{Expansiveness and no conjugate points}

In the 1980 's, R. Mañé made the following conjecture inspired by Klingenberg's Theorem (Theorem 3.5 in Chapter 3):

Let $(M, g)$ be a compact Riemannian manifold whose geodesic flow is expansive. Then $(M, g)$ has no conjugate points.

The conjecture was proved by M. Paternain [73] for surfaces. The proof has two main ideas which do not generalize to higher dimensions. The first one is the existence of stable and unstable sets for expansive homeomorphisms of surfaces, proved independently by Lewowicz [62] and Hiraide.

Let $f: X \longrightarrow X$ be a homeomorphism defined in a metric space $X$. Inspired by the definitions of local stable and unstable sets in Chapters 2, 3 , given $a>0$ we define the $a$-local stable set of $p$ as

$$
W_{a}^{s}(p)=\left\{q \in M, d\left(f^{n}(p), f^{n}(q)\right) \leq a \forall n \geq 0\right\} .
$$

The $a$-local unstable set of $p$ is given by

$$
W_{a}^{u}(p)=\left\{q \in M, d\left(f^{n}(p), f^{n}(q)\right) \leq a \forall n \leq 0\right\} .
$$

The following result is a version for expansive homeomorphisms of the existence of invariant submanifolds in hyperbolic dynamics (Theorem 2.1).

Theorem 5.1. [62] Let $f: S \longrightarrow S$ be an $\epsilon$-expansive homeomorphism of a compact surface $S$. Then for every point $p \in S$ the sets $W_{\epsilon}^{s}(p), W_{\epsilon}^{s}(p)$ are continuous curves which have a local product structure at every point of $S$ but at a finite number of periodic orbits. These exceptional orbits behave like prone singularities of pseudo-Anosov homeomorphisms of surfaces. Moreover, every expansive homeomorphism of a compact surface is conjugate to a pseudo-Anosov homeomorphism.

For the definition of pseudo-Anosov homeomorphisms and prone singularities we refer to [99]. According to the definition of local product structure given in Chapter 2, we have that the collections of stable and unstable sets behave like a pair of foliations with singularities. Outside the singularities each point $p$ has an open neighborhood $U(p)$ where the stable and unstable sets determine a continuous chart $\psi: U(p) \longrightarrow(-1,1) \times(-1,1)$. This chart satisfies the following two properties:

1. $\psi(p)=(0,0)$.

2. $\psi^{-1}\left((-1,1) \times\left\{y_{0}\right\}\right)$ is the connected component of $W_{\epsilon}^{s}\left(\psi^{-1}\left(x_{0}, y_{0}\right)\right) \cap$ $U(p)$ containing $\psi^{-1}\left(x_{0}, y_{0}\right)$ for every $y_{0} \in(-1,1)$.

3. $\psi^{-1}\left(\left\{x_{0}\right\} \times(-1,1)\right)$ is the connected component of $W_{\epsilon}^{u}\left(\psi^{-1}\left(x_{0}, y_{0}\right)\right) \cap$ $U(p)$ containing $\psi^{-1}\left(x_{0}, y_{0}\right)$ for every $x_{0} \in(-1,1)$. 
We have then a very good topological description of the dynamics of expansive homeomorphisms of compact surfaces. Such homeomorphisms are almost Anosov systems from the topological point of view. They fail to be topologically Anosov in a finite set of periodic orbits. This structure is generalized without much problems to expansive flows without singularities in three dimensional manifolds.

The second important idea is the application of some tools of the theory of codimension one foliations, which allow to show that there are no prone singularities and that the central leaves are graphs of the canonical projection. We recommend the reader to look at [73] for details.

The conjecture remains open in higher dimensions. We shall show a partial result at the end of the chapter.

\section{Expansive geodesic flows without conjugate points in higher dimensions}

We shall see in this section how qualitative methods to study geodesics in manifolds without conjugate points can provide deep knowledge about the dynamics and the global geometry of expansive geodesic flows. The purpose of the section is to show the highlights of the proof of the following result found in [86].

Theorem 5.2. Let $M$ be a compact manifold without conjugate points. Suppose that the geodesic flow is expansive. Then the following properties hold:

1. If a pair of geodesics $\gamma, \beta$ in $(\tilde{M}, g)$ with $d(\gamma, \beta) \leq D$ for some constant $D>0$, we have that $\gamma=\beta$. Here, $d(\gamma, \beta)$ is the Hausdorff distance.

2. Geodesic rays diverge in the universal covering.

3. The map $\theta \rightarrow H_{\theta}(0)$ is continuous.

4. The sets $\tilde{\mathcal{F}}^{s}(\theta), \tilde{\mathcal{F}}^{u}(\theta)$ are true stable and unstable sets for every $\theta \in T_{1} \tilde{M}$, namely, each orbit starting at $\eta \in \tilde{\mathcal{F}}^{s}(\theta)$ satisfies $d\left(\phi_{t}(\eta), \phi_{t}(\theta)\right) \rightarrow 0$ as $t \rightarrow+\infty$.

5. The families of sets $\mathcal{F}^{s}, \mathcal{F}^{u}$ are continuous foliations having a local product structure.

We shall subdivide the proof in many steps. 


\subsection{Expansiveness implies quasi-convexity and asymp- toticity}

Let us start with the following result, which is inspired by Lemma 2.4 in the work of Walters [103].

Lemma 5.1. Let $M$ be a compact manifold having no conjugate points and let $\phi_{t}: T_{1} M \longrightarrow T_{1} M$ be $\epsilon$-expansive. Then, given $0<\delta \leq \epsilon$ there exists $n=n(\delta) \in \mathbb{N}$ such that for every pair of geodesics $\gamma, \beta$ in $\tilde{M}$ satisfying

$$
\sup \{d(\gamma(t), \beta(t)), d(\gamma(s), \beta(s))\} \leq \frac{1}{n}
$$

for some $t<s$ then we have that

$$
d_{H}(\gamma([t, s]), \beta([t, s])) \leq \delta,
$$

where $d_{H}$ is the Hausdorff distance.

Proof. The proof of the lemma goes pretty much as the proof of Lemma 2.4 for expansive homeomorphisms of compact manifolds. However, Lemma 5.1 is not a straightforward consequence of Lemma 2.4 applied to the time one map of the geodesic flow in $T_{1} M$. The point is that the assumptions of Lemma 5.1 involve the distance in $\tilde{M}$ and not in $T_{1} M$, and nearby points in different geodesics in $\tilde{M}$ might not be close to each other in $T_{1} M$.

We argue by contradiction. Suppose that the statement is not true. Then there exists some $\delta>0$ such that for every $n \in \mathbb{N}$ there is a pair of geodesics $\gamma_{n}, \beta_{n}$, and numbers $s_{n}<t_{n}$ satisfying

1. $\sup \left\{d\left(\gamma_{n}\left(s_{n}\right), \beta_{n}\left(s_{n}\right)\right), d\left(\gamma_{n}\left(t_{n}\right), \beta_{n}\left(t_{n}\right)\right)\right\} \leq \frac{1}{n}$,

2. $d_{H}\left(\gamma_{n}\left(\left[s_{n}, t_{n}\right]\right), \beta_{n}\left(\left[s_{n}, t_{n}\right]\right)\right) \geq \delta$.

Let us join $\gamma_{n}\left(s_{n}\right), \beta_{n}\left(s_{n}\right)$ with the geodesic $\left[\gamma_{n}\left(s_{n}\right), \beta_{n}\left(s_{n}\right)\right]$, and join $\gamma_{n}\left(t_{n}\right), \beta_{n}\left(t_{n}\right)$ with the geodesic $\left[\gamma_{n}\left(t_{n}\right), \beta_{n}\left(t_{n}\right)\right]$. By the continuity of geodesics in $\tilde{M}$ with respect to boundary conditions (recall that geodesics in $\tilde{M}$ are unique minimizers), we can find points $a_{n} \in\left[\gamma_{n}\left(s_{n}\right), \beta_{n}\left(s_{n}\right)\right]$, $b_{n} \in\left[\gamma_{n}\left(t_{n}\right), \beta_{n}\left(t_{n}\right)\right]$, such that

$$
d_{H}\left(\gamma_{n}\left(\left[s_{n}, t_{n}\right]\right),\left[a_{n}, b_{n}\right]\right)=\delta .
$$

So we can assume without loss of generality that $\beta_{n}\left(\left[s_{n}, t_{n}\right]\right)=\left[a_{n}, b_{n}\right]$, and let $x_{n}$ be such that $d\left(\gamma_{n}\left(x_{n}\right), \beta_{n}\left(\left[s_{n}, t_{n}\right)\right)=\delta\right.$. It is clear that if $n \rightarrow$ $+\infty$, then $\left|s_{n}-x_{n}\right| \rightarrow+\infty$ and $\left|t_{n}-x_{n}\right| \rightarrow+\infty$, by the continuity of geodesics with respect to initial conditions.

Consider the geodesics $\Gamma_{n}(t)=\gamma_{n}\left(t+x_{n}\right), \Upsilon_{n}(t)=\beta_{n}\left(t+x_{n}\right)$ restricted to the intervals $\left[s_{n}-x_{n}, t_{n}-x_{n}\right]$. This interval contains $t=0$ where $\Gamma_{n}(0)=\gamma_{n}\left(x_{n}\right), \Upsilon_{n}(0)=\beta_{n}\left(x_{n}\right)$. 
By taking covering isometries in $\tilde{M}$, we can suppose that the points $\Gamma_{n}(0)$ belong to a compact fundamental domain $M_{0}$ of $M$. So we get a convergent subsequence of geodesics $\Gamma_{n_{k}} \rightarrow \Gamma_{\infty}, \Upsilon_{n_{k}} \rightarrow \Upsilon_{\infty}$, such that

1. $\Gamma_{\infty} \neq \Upsilon_{\infty}$,

2. $d_{H}\left(\Gamma_{\infty}, \Upsilon_{\infty}\right) \leq \delta$.

This contradicts the expansiveness assumption.

The above observation has strong consequences in the global geometry of geodesics in $\tilde{M}$. The first one is quasi-convexity.

Corollary 5.1. Let $(M, g)$ be compact without conjugate points and expansive geodesic flow. Then $(\tilde{M}, \tilde{g})$ is quasi-convex.

Proof. Let $[a, b],[p, q]$ be two geodesic segments in $\tilde{M}$ and assume without loss of generality that $d(a, p) \geq d(b, q)$. Let $n_{0}$ be as in Lemma 5.1 and let $N_{0}=\left[\left[d(a, p) n_{0}\right]\right]$, where $[[r]]$ is the integer part of the number $r$. Let us make a partition of the geodesic segments

$$
\begin{aligned}
{[a, p] } & =\bigcup_{i=0}^{N_{0}}\left[a_{i}, a_{i+1}\right] \\
{[b, q] } & =\bigcup_{i=0}^{N_{0}}\left[b_{i}, b_{i+1}\right]
\end{aligned}
$$

with $a_{0}=a, b_{0}=b, a_{N_{0}+1}=p, b_{N_{0}+1}=q$ such that

1. length $\left(\left[a_{i}, a_{i+1}\right]\right)=\frac{1}{n_{0}}$ for every $0 \leq i \leq N_{0}$ and length $\left(\left[a_{N_{0}}, a_{N_{0}+1}\right]\right)$ $\leq \frac{1}{n_{0}}$ (notice that $a_{N_{0}}$ may be equal to $a_{N_{0}+1}$ ).

2. length $\left(\left[b_{i}, b_{i+1}\right]\right)=\frac{d(b, q)}{N_{0}+1} \leq \frac{1}{n_{0}}$ for every i.

From Lemma 5.1 and properties 1, 2 above we deduce that

$$
\begin{aligned}
d([a, b],[p, q]) & \leq \sum_{i=0}^{N_{0}} d\left(\left[a_{i}, b_{i}\right],\left[a_{i+1}, b_{i+1}\right]\right) \\
& \leq\left(N_{0}+1\right) \delta\left(n_{0}\right) \\
& =\delta\left(n_{0}\right)\left(\left[\left[n_{0} d(a, p)\right]\right]+1\right) \\
& \leq \delta\left(n_{0}\right) n_{0} d(a, p)+\delta\left(n_{0}\right) \\
& =K \sup (d(a, p), d(b, q))+C
\end{aligned}
$$

where $K=\delta\left(n_{0}\right) n_{0}$ and $C=\delta\left(n_{0}\right)$. This finishes the proof of the lemma.

Corollary 5.2. Let $(M, g)$ be a compact manifold with expansive geodesic flow. Then, there exists $K, C>0$ such that for every geodesic $\gamma \subset \tilde{M}$ and every Busemann asymptote of $\gamma, \beta$, we have

$$
d_{H}(\alpha([0,+\infty)), \beta([0,+\infty)) \leq K d(\gamma(0), \beta(0))+C .
$$

Proof. This follows from the quasi convexity of $(\tilde{M}, \tilde{g})$ and Lemma 4.6. 


\subsection{Strong stable and unstable sets}

The stable set of the geodesic flow at $\theta \in T_{1} \tilde{M}$ is defined by

$$
W^{s}(\theta)=\left\{x \in T_{1} \tilde{M}, d\left(\phi_{t}(x), \phi_{t}(\theta)\right) \leq C(x, \theta) \forall t \geq 0\right\} .
$$

The unstable set of $\theta \in T_{1} \tilde{M}$ is defined by

$$
W^{u}(p)=\left\{x \in T_{1} \tilde{M}, d\left(\phi_{t}(x), \phi_{t}(\theta)\right) \leq D(x, \theta) \forall t \leq 0\right\} .
$$

Notice that the above definition is slightly more general than the definition of $a$-local stable and unstable sets. The constants $C(x, \theta), D(x, \theta)$ might depend on $x, \theta$, while in the case of $a$-local stable and unstable sets, we took $a=C(x, \theta)=D(x, \theta)$. Given $\theta \in T_{1} \tilde{M}$, let us consider the sets $\tilde{\mathcal{F}}^{s}(\theta), \tilde{\mathcal{F}}^{u}(\theta)$ defined in Chapter 4, at the end of Section 4.1. Recall that $\tilde{\mathcal{F}}^{s}(\theta)$ is the set of vectors which are opposite to the gradients of the Busemann function of $\theta$ at the points of $H_{\theta}(0)$. So, according to Corollary 5.2, we have that $\tilde{\mathcal{F}}^{s}(\theta)$ is a subset of the stable set of $\theta$. Analogously, $\mathcal{F}^{u}(\theta)$ is a subset of the unstable set of $\theta$. The question is: are there more points in the stable and unstable sets of $\theta$ ? The answer is no:

Proposition 5.1. Let $(M, g)$ be a compact manifold without conjugate points whose geodesic flow is expansive. Then

$$
\begin{aligned}
& W^{s}(\theta)=\tilde{\mathcal{F}}^{s}(\theta), \\
& W^{u}(\theta)=\tilde{\mathcal{F}}^{u}(\theta),
\end{aligned}
$$

for every $\theta \in T_{1} \tilde{M}$.

Proposition 5.1 is in fact a generalization of Lemma 2.5, which states the topological contraction of local stable sets of expansive homeomorphisms with local product structure of compact manifolds. However, Proposition 5.1 is not just purely dynamical. It involves the issue of the existence and uniqueness of asymptotic geodesics and their relationship with Busemann asymptotes, discussed in Chapter 4 . We shall subdivide the proof in many steps. The first one is another application of Lemma 5.1. 
Lemma 5.2. Let $(M, g)$ be compact without conjugate points, with expansive geodesic flow. If $\gamma, \beta$ are two geodesics in $\tilde{M}$ satisfying

$$
d_{H}(\gamma, \beta) \leq C
$$

where $d_{H}(\gamma, \beta)$ is the Hausdorff distance - then there exists $t_{0}$ such that $\gamma(t)=\beta\left(t+t_{0}\right)$.

Proof. Let $\epsilon$ be the the expansiveness constant of the geodesic flow. It is clear that there exists $\bar{\epsilon}$ such that two geodesic segments of length greater than 1 in M have Hausdorff distance less than $\bar{\epsilon}$ if and only if their Hausdorff distance in $T_{1} \tilde{M}$ as pieces of orbits of the geodesic flow is less than $\epsilon$, so we shall assume without loss of generality that $\bar{\epsilon}=\epsilon$. Now, let us assume that the Hausdorff distance between $\gamma$ and $\beta$ is bounded above by $\mathrm{C}$. Then these geodesics are bi-asymptotic and there exists $C^{\prime}>0$ such that

$$
d(\gamma(t), \beta(t)) \leq C^{\prime}
$$

for every $t \in R$. Consider the geodesic segments $\sigma_{m}=[\gamma(m), \beta(m)]$. All their lengths are less than or equal to C'.

Let $\delta=\epsilon, n=n(\epsilon)>0$ be the numbers defined in Lemma 5.1. Let us take $m>0$ and let us suppose without loss of generality that the length of $\sigma_{m}$ is greater than or equal to the length of $\sigma_{-m}$. Let us parametrize $\sigma_{m}:\left[0, a_{m}\right] \longrightarrow \tilde{M}$, for $m>0$, by arc length in a way that $\sigma_{m}(0)=\gamma(m)$, $\sigma_{m}\left(a_{m}\right)=\beta(m)$. We can choose a partition $0=t_{0}^{m}<t_{1}^{m}<. .<t_{k_{m}}^{m}=a_{m}$ of $\left[0, a_{m}\right]$ such that $t_{i+1}^{m}-t_{i}^{m}=\frac{1}{n}$, for every $0 \leq i \leq k_{m}-2$, and $a_{m}-$ $t_{k_{m}-1}^{m} \leq \frac{1}{n}$. Let us consider a partition $0=s_{0}^{m}<s_{1}^{m}<. .<s_{k_{m}}^{m}=a_{-m}$ of $\left[0, a_{-m}\right]$ such that $s_{i+2}^{m}-s_{i+1}^{m}=s_{i+1}^{m}-s_{i}^{m}$ for every $0 \leq i \leq k_{m}-3$, and $a_{-m}-s_{k_{m}-1}^{m}=a_{m}-t_{k_{m}-1}^{m}$. In this way we get

$$
s_{i+1}^{m}-s_{i}^{m} \leq t_{i+1}^{m}-t_{i}^{m}=\frac{1}{n}
$$

for every $0 \leq i \leq k_{m}-1$, because we are assuming that $\sigma_{m}$ is larger than $\sigma_{-m}$, or equivalently, $a_{-m} \leq a_{m}$. Observe now that similar partitions can be obtained for $\left[0, a_{m}\right],\left[0, a_{-m}\right]$ assuming $a_{m} \leq a_{-m}$, just interchanging the roles of $a_{m}, a_{-m}$, so we can suppose without loss of generality that $a_{-m} \leq a_{m}$ for every $m>0$.

Consider now the geodesic segments

$$
\alpha_{m}^{i}=\left[\sigma_{-m}\left(s_{i}^{m}\right), \sigma_{m}\left(t_{i}^{m}\right)\right]
$$

for every $0 \leq i \leq k_{m}$. From the construction we get that

$$
d_{H}\left(\alpha_{m}^{i}, \alpha_{m}^{i+1}\right) \leq \delta \leq \epsilon
$$


for every $0 \leq i \leq k_{m}$ and $m \geq 1$. By the choice of $k_{m}$, we have that

$$
k_{m} \frac{1}{n} \leq C^{\prime}
$$

which implies that $k_{m} \leq n C^{\prime}=A$. Since $\tilde{M}$ is quasi-convex, there exists $B>0$ such that the Haussdorff distance from $\alpha_{m}^{i}$ to $\gamma$ is bounded above by $B$. So taking convergent subsequences of the segments $\alpha_{m}^{i}$ as $m$ goes to $\infty$ we can obtain a collection of at most $A+1$ geodesics $\alpha^{i}, i=0,1, . ., A^{\prime}$, where $\alpha^{0}=\gamma, \alpha^{A^{\prime}}=\beta$, satisfying

$$
d_{H}\left(\alpha^{i}, \alpha^{i+1}\right) \leq \epsilon
$$

for every $i$. But from the definition of expansiveness we get that $\gamma=\alpha^{i}=\beta$ for every $i$, thus proving the lemma.

Observe that Lemma 5.2 tells us that the expansiveness of the geodesic flow implies a sort of universal expansiveness in $\tilde{M}$ : the geodesic flow in $T_{1} \tilde{M}$ is expansive for every constant $C>0$. This implies that

Corollary 5.3. Let $(M, g)$ be compact without conjugate points and expansive geodesic flow. Then two asymptotic geodesics are strongly asymptotic. Namely, if $d(\gamma(t), \beta(t)) \leq B$ for every $t \geq 0$, then there exists $t_{0} \in \mathbb{R}$ such that

$$
\lim _{t \rightarrow+\infty} d\left(\gamma(t), \beta\left(t_{0}+t\right)\right)=0 .
$$

Proof. This is more or less the same argument of the proof of Lemmas 2.5 and 5.2. In fact, if we had two asymptotic geodesics $\gamma, \beta$ in $\tilde{M}$ and $a>0$ such that

$$
\limsup _{t \geq 0} d(\gamma(t), \beta) \geq a,
$$

then we can obtain a sequence of pairs of geodesics converging to a pair of different bi-asymptotic geodesics. This contradicts Lemma 5.2.

The following shortcut result due to Morse [69] is the last step before the proof of Proposition 5.1.

Lemma 5.3. Let $(M, g)$ be a complete manifold. Given $\alpha>0$ there exists $\delta>0$ such that for every pair of globally minimizing geodesics $\gamma_{(p, v)}, \gamma_{(p, w)}$ in $\tilde{M}$, such that the angle formed by $v, w$ is at least $\alpha$, we have

$$
\begin{aligned}
& d\left(\gamma_{(p, v)}(t), \gamma_{(p, w)}\right) \geq \delta, \\
& d\left(\gamma_{(p, w)}(t), \gamma_{(p, v)}\right) \geq \delta,
\end{aligned}
$$

for every $t \geq 1$. 
The proof is very simple, the reader may look at Morse's work [69] or take it as an exercise.

\section{Proof of Proposition 5.1}

Let $\gamma_{\theta} \subset \tilde{M}$ be a geodesic, and let $\gamma_{(x, w)}$ be asymptotic to $\gamma_{\theta}$. We must show that $\gamma_{(x, w)}$ is a Busemann asymptote of $\gamma_{\theta}$. We already know that there is a Busemann asymptote $\gamma_{(x, v)}$ of $\gamma_{\theta}$ through $x$. If $(x, v) \neq(x, w)$, then we can apply Lemma 5.3 to deduce that there is $\delta>0$ such that

$$
\begin{aligned}
& d\left(\gamma_{(x, v)}(t), \gamma_{(p, w)}\right) \geq \delta, \\
& d\left(\gamma_{(x, w)}(t), \gamma_{(p, v)}\right) \geq \delta,
\end{aligned}
$$

for every $t \geq 1$. By considering iterates of this pair of geodesics by covering isometries of the fundamental group of $M$, the same limiting argument used in the proof of Lemma 5.2 gives us a pair of different, bi-asymptotic geodesics in $\tilde{M}$. This contradicts Lemma 5.2 , so $(x, v)=(x, w)$, and the Busemann asymptote $\gamma_{(x, v)}$ is the only geodesic asymptotic to $\gamma_{\theta}$ containing $x$.

Corollary 5.4. Let $(M, g)$ be a compact manifold without conjugate points and expansive geodesic flow. Then the map $\theta \rightarrow H_{\theta}(0)$ is continuous.

Proof. By Proposition 5.1, given $\theta \in T_{1} \tilde{M}$ and $x \in \tilde{M}$ there exists a unique geodesic asymptotic to $\gamma_{\theta}$ through $x$. This is just Pesin's asymptoticity axiom (Definition 4.7), which implies the continuity of horospheres.

We get as corollary as well the uniform (topological) contraction of the stable sets.

Corollary 5.5. Let $M$ be a compact Riemannian manifold with no conjugate points whose geodesic flow is expansive. Then given $0<r<D$ there exists $T>0$ such that for every $\theta=(p, v) \in T_{1} \tilde{M}$ we have

$$
\psi_{t}^{\theta}\left(B_{D}(p) \bigcap H_{\theta}(0)\right) \subset B_{r}\left(\psi_{t}^{\theta}(p)\right) \bigcap H_{\left(\gamma_{\theta}(t), \gamma_{\theta}^{\prime}(t)\right)}(0),
$$

for every $t \geq T$, where $B_{r}(p)$ is the ball of radius $r$ centered at $p$, and $\psi_{t}^{\theta}$ is, as in Chapter 4, the Busemann flow of $\theta$. Moreover, we have that

$$
W^{s}(\theta) \cap W^{u}(\theta)=\{\theta\}
$$

for every $\theta \in T_{1} \tilde{M}$. 


\section{Divergence of geodesic rays in $\tilde{M}$}

The divergence of geodesic rays in $\tilde{M}$ is closely related with the existence of strong stable sets. Actually, we can get the divergence of geodesic rays by applying Corollary 5.4 and Theorem 4.3. However, we can prove the divergence as a consequence of the contraction of stable sets.

Lemma 5.4. Let $(M, g)$ be a compact manifold without conjugate points and expansive geodesic flow. Then geodesic rays diverge uniformly in $\tilde{M}$.

Proof. Assume by contradiction that there is no uniform divergence. Then there exist $\alpha>0, b>0$, such that for every $n \in \mathbb{N}$, there exist geodesics $\gamma_{\left(p_{n}, v_{n}\right)}, \gamma_{\left(p_{n}, w_{n}\right)}$ in $\tilde{M}$, satisfying

1. $\measuredangle\left(v_{n}, w_{n}\right) \geq \alpha$ for every $n \in \mathbb{N}$.

2. There exist $t_{n}, s_{n} \rightarrow+\infty$ such that

$$
d\left(\gamma_{\left(p_{n}, v_{n}\right)}\left(t_{n}\right), \gamma_{\left(p_{n}, w_{n}\right)}\left(s_{n}\right)\right) \leq b
$$

By quasi convexity, we get

$$
d\left(\gamma_{\left(p_{n}, v_{n}\right)}(t), \gamma_{\left(p_{n}, w_{n}\right)}(t)\right) \leq b
$$

for every $t \in\left[0, \min \left\{t_{n}, s_{n}\right\}\right]$. By Morse's shortcut Lemma (Lemma 5.3) there exists $\delta>0$ such that

$$
\begin{aligned}
& d\left(\gamma_{\left(p_{n}, v_{n}\right)}(t), \gamma_{\left(p_{n}, w_{n}\right)}\right) \geq \delta, \\
& d\left(\gamma_{\left(p_{n}, w_{n}\right)}(t), \gamma_{\left(p_{n}, v_{n}\right)}\right) \geq \delta,
\end{aligned}
$$

for every $t \geq 1$. Since $\min \left\{t_{n}, s_{n}\right\} \rightarrow+\infty$ with $n$, we apply the same limiting argument in the proof of Lemma 5.2 to iterates of $\gamma_{\left(p_{n}, v_{n}\right)}, \gamma_{\left(p_{n}, w_{n}\right)}$ by covering isometries. So we get a pair of different, bi-asymptotic geodesics in $\tilde{M}$ which contradicts Lemma 5.2. This finishes the proof of the lemma.

We saw all the information about the global geometry of geodesics in $\tilde{M}$ that we got from the expansiveness assumption, that is purely topological. In the next chapter we shall give some other applications of $C^{0}$ methods to study the fundamental group of manifolds with expansive geodesic flow.

\section{Proof of Theorem 5.2 and applications}

Items (1), (2), (3), (4) in Theorem 5.2 follow from the results of the previous sections. Item (5), the local product structure, is not very difficult to show and we just give an outline of the proof. 
Given $\theta=(p, v) \in T_{1} \tilde{M}$, let $\delta>0$ be such that the ball $B_{\delta}(z)$ of radius $\delta$ centered at $z \in M$ is embedded for every $z \in M$. Let $\tilde{\mathcal{F}}_{\delta}^{s}(\theta)$ be the set

$$
\tilde{\mathcal{F}}_{\delta}^{s}(\theta)=\left\{(x, w) \in \tilde{F}^{s}(\theta), d(x, p)<\delta\right\} .
$$

This is a $C^{0}, n-1$ dimensional subset of $T_{1} M$, since it is homeomorphic to the ball $B_{\delta}(p)$. Let

$$
\tilde{\mathcal{F}}_{\delta}^{u}(\theta)=\left\{(x, w) \in \tilde{F}^{u}(\theta), d(x, p)<\delta\right\} .
$$

If $\Pi: T_{1} \tilde{M} \longrightarrow T_{1} M$ is the projection $\Pi(p, v)=\left(\pi(p), D_{p} \pi(v)\right)$, where $\pi: \tilde{M} \longrightarrow M$ is the covering map, then the sets $\operatorname{Pi}\left(\tilde{\mathcal{F}}_{\delta}^{s}(\theta)\right), \Pi\left(\tilde{\mathcal{F}}_{\delta}^{u}(\theta)\right.$ are open subsets (in the relative topology) of local stable and unstable sets respectively for $\pi(p, v)$ in $T_{1} M$.

Consider the set

$$
S(\theta)=\bigcup_{\sigma \in \tilde{\mathcal{F}}_{\delta}^{s}(\theta)}\left\{\phi_{t}\left(\tilde{\mathcal{F}}_{\delta}^{u}(\sigma)\right),|t|<\delta\right\},
$$

that is an open neighborhood of $\theta$ in $T_{1} \tilde{M}$ because of Brower's invariance of domain theorem. The set $S(\theta)$ is foliated by the local center unstable sets $\left\{\phi_{t}\left(\tilde{\mathcal{F}}_{\delta}^{u}(\sigma)\right),|t|<\delta\right\}$. Let us define

$$
U(\theta)=\bigcup_{\sigma \in \tilde{\mathcal{F}}_{\delta}^{u}(\theta)}\left\{\phi_{t}\left(\tilde{\mathcal{F}}_{\delta}^{s}(\sigma)\right),|t|<\delta\right\},
$$

again an open neighborhood of $\theta$ in $T_{1} \tilde{M}$. This set is foliated by the local center stable sets $\left\{\phi_{t}\left(\tilde{\mathcal{F}}_{\delta}^{s}(\sigma)\right),|t|<\delta\right\}$, and then the intersection $B(\theta)=$ $S(\theta) \cap U(\theta)$ is an open neighborhood of $\theta$ where local center stable and local unstable sets intersect by the continuity of the stable and unstable foliations.

By the expansiveness of the geodesic flow, the center stable set of $\theta$ and the center unstable set of $\theta$ just meet at the orbit of $\theta$. Now, it is not difficult to show that there exists a local transversal section of the geodesic flow at $\theta$ where the intersections of the local center stable sets and the local center unstable sets give a local product structure. For the details we refer to [86]. The same configuration of stable and unstable sets occurs in $T_{1} M$ after applying the projection $\Pi$. This finishes the proof of Theorem 5.2.

We would like to finish the section with some applications of Theorem 5.2. Combining the local product structure with the stability results of Chapter 2 we get (see [86]), 
Corollary 5.6. Expansive geodesic flows in compact manifolds without conjugate points are topologically stable and have the pseudo-orbit tracing property. In particular, the Anosov closing lemma holds and closed orbits are dense. Moreover, the geodesic flow is topologically transitive.

The next application is in [85], and is a partial answer to Mañé's conjecture relating expansiveness and conjugate points.

Theorem 5.3. Let $(M, g)$ be compact without conjugate points and expansive geodesic flow with expansiveness constant $\epsilon>0$. There exists an open $C^{1}$ neighborhood $U(g)$ of the metric $g$ of $C^{\infty}$ metrics in $M$, and $\epsilon^{\prime}>0$, such that if $(M, h) \in U(g)$ has expansive geodesic flow with expansiveness constant $\epsilon^{\prime}$ then $(M, h)$ has no conjugate points.

Proof. Since the geodesic flow $\phi_{t}$ of $(M, g)$ is topologically stable, there exists an $C^{1}$ open neighborhood $U(g)$ of the metric where the geodesic flow $\psi_{t}$ of a metric $h$ in $U(g)$ is semi-conjugate to $\phi_{t}$. According to the results in Chapter 2, there exists $\epsilon^{\prime}>0$ such that if $\psi_{t}$ is $\epsilon^{\prime}$-expansive then the semiconjugacy is actually a continuous conjugacy $f:\left(T_{1} M, h\right) \longrightarrow\left(T_{1} M, g\right)$. Since $f$ is a homeomorphism and the closed orbits of $\phi_{t}$ are dense, the closed orbits of $\psi_{t}$ are also dense.

Claim: Each closed geodesic in $(M, g)$ is unique in its free homotopy class.

This follows from the fact that two closed geodesics in a homotopy class of $M$ lift to $\tilde{M}$ to pairs of bi-asymptotic geodesics. And by Lemma 5.2 there are no such geodesics.

Hence, the Claim holds in $(M, h)$ too, because of the existence of the homeomorphism $f$. Since in each non trivial homotopy class there exists at least one which minimizes the length in the class, we get that all closed geodesics of $(M, h)$ are minimizers in their corresponding homotopy classes. Therefore, closed geodesics in $(M, g)$ have no conjugate points. And since the set of closed geodesics is dense in $\left(T_{1} M, h\right)$, we conclude that the whole set of $h$-geodesics has no conjugate points, because the set of geodesics without conjugate points is a closed subset of the set of geodesics. This finishes the proof of the theorem.

In the next chapter we shall mention some other special features of the global geometry of manifolds without conjugate points and expansive geodesic flows. The universal covering of such a manifold is what is called a Gromov hyperbolic space, a fact that has very strong implications in the global geometry of the manifold.

The theory of expansive geodesic flows in manifolds without conjugate points is one of our main motivations for the conjectures in the survey. 
They provide examples of higher dimensional, non Anosov systems with many of the most important properties of the topological dynamics and global geometry of Anosov geodesic flows. And it would be natural to ask whether compact manifolds without conjugate points and expansive geodesic flows admit metrics of negative curvature. We shall discuss this issue later in the context of three dimensional manifolds and the works of Perelmann about the Poincaré conjecture. 


\section{Chapter 6}

\section{The fundamental group, global geometry and geometric group theory}

In this chapter we continue to develop the employ of $C^{0}$, topological methods to study the relationships between the dynamics of the geodesic flow, the topology and the global geometry of the manifold. When we refer to the global geometry of a manifold $(M, g)$ we refer actually to the geometry of the universal covering $\tilde{M}$ endowed with the pullback $\tilde{g}$ of the metric $g$ by the covering map. We follow the notations of Chapter 1 .

\section{Fundamental group, algebra and geometry}

We start by recalling briefly some basic facts concerning the fundamental group of compact manifolds without conjugate points. As we observed in Chapter 1 , there is a natural representation of the point base fundamental groups with groups of covering isometries of $(\tilde{M}, \tilde{g})$, so we shall identify $\pi_{1}(M)$ with the group of covering isometries throughout the chapter. Our main references for this subject are [26], [88].

Lemma 6.1. Let $M$ be a compact manifold with no conjugate points. Then the following assertions hold:

1. Given a covering isometry $T$, the displacement function $d_{T}: \tilde{M} \longrightarrow$ $\mathbb{R}, d_{T}(x)=d(x, T(x))$, assumes a positive minimum. Moreover, the set of critical points of $d_{T}$ consists only of minimum points.

2. The critical set of $d_{T}$ is foliated by geodesics $\gamma \subset \tilde{M}$ satisfying $T(\gamma(t))=\gamma(t+c(T))$ for every $t \in \mathbb{R}$, where $c(T)$ is the minimum value of $d_{T}$ and coincides with the minimum length of a closed geodesic in the free homotopy class of $\Pi(\gamma)$. Such a geodesic is called 
an axis of $T$ and it is the lift of a closed geodesic of $M$ in the homotopy class of $\Pi(\gamma)$, where $\Pi: \tilde{M} \longrightarrow M$ is the covering map.

3. A geodesic $\gamma$ is an axis of $T$ if and only if it is an axis of $T^{n}$. We have $T^{n}(\gamma(t))=\gamma(t+n c(T))$ for every $t \in \mathbb{R}$.

4. A covering isometry $S$ commutes with a covering isometry $T$ if and only if $S$ permutes the axes of $T$.

5. If $S$ commutes with $T$, the displacement function $d_{S}$ is bounded along any axis of $T$.

We just give some ideas of the proof of Lemma 6.1 because its statement is quite eloquent. From the very definition of covering isometries (last section of Chapter 1) we know that given $p \in M$ and $\tilde{p} \in \Pi^{-1}(p)$, we obtain a representation of the point base fundamental group $\pi_{1}(M, p)$ by a subgroup of isometries. Each element of this representation is obtained by lifting closed paths in a fixed homotopy class in $\pi_{1}(M, p)$, in a way that all the lifts contain the point $\tilde{p}$.

So let us take $p$ in a closed geodesic $\gamma_{(p, v)}$ with nontrivial homotopy class, and a lift $\tilde{\gamma}_{(\tilde{p}, \tilde{v})}$ of $\gamma_{(p, v)}$ in $\tilde{M}$. Then there exists a covering isometry $T: \tilde{M} \longrightarrow \tilde{M}$ associated to $\gamma_{(p, v)}$ and $\tilde{p}$ which satisfies $T\left(\tilde{\gamma}_{(\tilde{p}, \tilde{v})}\right)=\tilde{\gamma}_{(\tilde{p}, \tilde{v})}$. Moreover, parametrizing $\gamma_{(p, v)}$ by arc length we have

$$
T\left(\tilde{\gamma}_{(\tilde{p}, \tilde{v})}(t)\right)=\tilde{\gamma}_{(\tilde{p}, \tilde{v})}\left(t+l\left(\gamma_{(p, v)}\right)\right),
$$

where $l\left(\gamma_{(p, v)}\right)$ is the minimum positive period of $\gamma_{(p, v)}$ (which coincides with the length of $\gamma_{(p, v)}$ of course). Since $\gamma_{(p, v)}$ minimizes length in its (free) homotopy class - by Birkhoff's Theorem (Theorem 1.4) and the absence of conjugate points (Lemma 4.1) - the displacement $d_{T}(x)=d(x, T(x))$ is minimal along $\tilde{\gamma}_{(\tilde{p}, \tilde{v})}$. Moreover, the displacement $d_{T}(x)$ is the length of a closed path in $M$ homotopic to $\gamma_{(p, v)}$, so it is minimal if and only if it equals the length of $\gamma_{(p, v)}$. Thus, any point where the displacement is minimal is contained in a lift of a closed curve $c$ with minimal length in the homotopy class of $\gamma_{(p, v)}$ and hence a geodesic. The above considerations are part of the proof of items (1),(2) in Lemma 6.1. The proof of the fact that all critical points of $d_{T}$ are minima requires a little more work, we refer the reader to [26] for the details of this proof and the proof of item (3). Items (4) and (5) are easy to check from the definitions and the fact that covering isometries preserve distances.

Item (2) implies that $\pi_{1}(M)$ has no torsion, because any nontrivial covering isometry preserves the lift in $\tilde{M}$ of a closed geodesic of $M$. Since every geodesic in $\tilde{M}$ is globally minimizing, a periodic point of a covering isometry would give rise to a closed minimizing geodesic in $\tilde{M}$, a contradiction. In particular, every nontrivial element of $\pi_{1}(M)$ has infinite order. 
We shall change for convenience the notation of covering isometries given in Chapter 1. Since every covering isometry preserves an axis $\bar{\gamma}$ in $\tilde{M}$, we shall often denote by $T_{\bar{\gamma}}$ the covering isometry whose critical displacement is the minimum positive period of $\Pi(\bar{\gamma})$.

The first studies of the fundamental group of manifolds without conjugate points are due to Busemann [22], who gave some hints about many analogies between such groups and groups of isometries of manifolds with nonpositive curvature. For instance, Busemann observed that covering isometries with bounded displacement in $\tilde{M}$ have in fact constant displacement and hence, all points in $\tilde{M}$ are critical and contained in axes of the covering isometry. Such axes are all bi-asymptotic, so this covering isometry looks like a translation in $\mathbb{R}^{n}$ which preserves a flow by parallel lines.

The above result has a sort of converse whose proof gives a flavor of geometric rigidity type results involving the action of covering isometries and geodesics in manifolds without conjugate points with no a priori restrictions on the curvature sign. The following lemma is proved in [89], we include its proof here for the sake of completeness.

Lemma 6.2. Let $M$ be a compact manifold without conjugate points. Let $\bar{\gamma}$ be the lift of a closed geodesic $\gamma$ in $M$ of minimum period $l(\gamma)$ which is an axis of $T_{\bar{\gamma}}$. A covering transformation $T$ in $\pi_{1}(M)$ commutes with $T_{\bar{\gamma}}$ if and only if $T(\bar{\gamma})$ is bi-asymptotic to $\bar{\gamma}$.

Proof. If $T$ commutes with $T_{\bar{\gamma}}$ then $T$ preserves the axes of $T_{\bar{\gamma}}$ by Lemma 6.1 (4). In particular, the geodesic $T(\bar{\gamma})$ is an axis of $T_{\bar{\gamma}}$ and the displacement $d_{T}$ is bounded along $\bar{\gamma}$ : there exists $L>0$ such that $d_{T}(\bar{\gamma}(t)) \leq L$ for every $t \in \mathbb{R}$. But $d_{T}(\bar{\gamma}(t))=d(\bar{\gamma}(t), T(\bar{\gamma}(t))) \leq L$ for every $t \in \mathbb{R}$, and thus $\bar{\gamma}$, $T(\bar{\gamma}$ are bi-asymptotic.

To show the converse, suppose that $\bar{\gamma}$ and $T(\bar{\gamma})$ are bi-asymptotic. There exists $B>0$ such that $d_{H}(T(\bar{\gamma}), \bar{\gamma}) \leq B$, where $d_{H}$ is the Haussdorff distance between the geodesics. Let $A=d(\bar{\gamma}(0), T(\bar{\gamma}(0)))$, and let $l>0$ be the minimum period of $\gamma$.

Claim 1: $d(\bar{\gamma}(n l), T(\bar{\gamma}(n l))) \leq A+2 B$, for every $n \in Z$. In particular, the displacement of $T$ restricted to $\bar{\gamma}$ is bounded above.

For, let $t_{n} \in R$ be a point where the distance from $T(\gamma(n l))$ to $\gamma$ is attained, i.e., $d(T(\gamma(n l)), \gamma)=d\left(T(\gamma(n l)), \gamma\left(t_{n}\right)\right)$. On the one hand, by the triangle inequality and the fact that $T$ is an isometry, we have

$$
\begin{aligned}
d\left(\gamma(0), \gamma\left(t_{n}\right)\right) \leq & d(\gamma(0), T(\gamma(0)))+d(T(\gamma(0)), T(\gamma(n l))) \\
& +d\left(T(\gamma(n l)), \gamma\left(t_{n}\right)\right) \\
\leq & A+n l+B
\end{aligned}
$$


which implies that

$$
d\left(\gamma(0), \gamma\left(t_{n}\right)\right)-n l \leq A+B .
$$

On the other hand, by the same reason we have

$$
n l=d(T(\gamma(0)), T(\gamma(n l))) \leq A+B+d\left(\gamma(0), \gamma\left(t_{n}\right)\right),
$$

and hence

$$
n l-d\left(\gamma(0), \gamma\left(t_{n}\right)\right) \leq A+B .
$$

Therefore, we conclude that

$$
\left|d\left(\gamma(0), \gamma\left(t_{n}\right)\right)-n l\right| \leq A+B .
$$

Now, it is not difficult to show that $\gamma\left(t_{n}\right)$ cannot be too far from $\gamma(n l)$. In fact, assume first that $t_{n} \leq n l$. Then

$$
d(\gamma(0), \gamma(n l))=d\left(\gamma(0), \gamma\left(t_{n}\right)\right)+d\left(\gamma\left(t_{n}\right), \gamma(n l)\right),
$$

which, by the above inequality, implies that

$$
n l \geq n l-(A+B)+d\left(\gamma\left(t_{n}\right), \gamma(n l)\right) .
$$

Thus, $d\left(\gamma\left(t_{n}\right), \gamma(n l)\right) \leq A+B$. Assuming that $t_{n} \geq n l$, an analogous reasoning leads to the same conclusion. Hence,

$$
\begin{aligned}
d(\gamma(n l), T(\gamma(n l))) & \leq d\left(\gamma(n l), \gamma\left(t_{n}\right)\right)+d\left(\gamma\left(t_{n}\right), T(\gamma(n l))\right) \\
& \leq A+B+B=A+2 B,
\end{aligned}
$$

as stated in the Claim.

Claim 2: There exists $k \in Z$ such that $T T_{\bar{\gamma}}^{k}=T_{\bar{\gamma}}^{k} T$.

In other words, there exists a power of $T_{\bar{\gamma}}$ that commutes with $T$. First of all, by Claim 1, we have that

$$
\begin{aligned}
d(\gamma(n l), T(\gamma(n l))) & =d\left(T_{\bar{\gamma}}^{n}(\gamma(0)), T T_{\bar{\gamma}}^{n}(\gamma(0))\right) \\
& \left.=d(\gamma(0)), T_{\bar{\gamma}}^{-n} T T_{\bar{\gamma}}^{n}(\gamma(0))\right) \\
& \leq A+2 B,
\end{aligned}
$$

for every $n \in Z$. And then, since the action of the isometries of $\pi_{1}(M)$ is discrete in $\tilde{M}$, there are only a finite number of different conjugates of $\gamma(0)$ of the type $T_{\bar{\gamma}}^{-n} T T_{\bar{\gamma}}^{n}(\gamma(0))$. So there exist $0<n_{1}<n_{2}$ such that

$$
T_{\bar{\gamma}}^{-n_{1}} T T_{\bar{\gamma}}^{n_{1}}(\gamma(0))=T_{\bar{\gamma}}^{-n_{2}} T T_{\bar{\gamma}}^{n_{2}}(\gamma(0)),
$$

which implies that

$$
T_{\bar{\gamma}}^{-n_{1}} T T_{\bar{\gamma}}^{n_{1}}=T_{\bar{\gamma}}^{-n_{2}} T T_{\bar{\gamma}}^{n_{2}}
$$


because $\pi_{1}(M)$ is torsion free. Hence,

$$
T=T_{\bar{\gamma}}^{-\left(n_{2}-n_{1}\right)} T T_{\bar{\gamma}}^{\left(n_{2}-n_{1}\right)},
$$

thus proving the Claim with $k=n_{2}-n_{1}$.

Claim 3: $T T_{\bar{\gamma}}=T_{\bar{\gamma}} T$.

Indeed, notice that the geodesic $\gamma_{1}=T(\bar{\gamma})$ is a lift of $\gamma$ with the same minimum period $l$. Let $T_{\gamma_{1}}$ be the translation with axis $\gamma_{1}$ and critical displacement $d_{T_{\gamma_{1}}}\left(\gamma_{1}(t)\right)=l$. Since $T_{\bar{\gamma}}^{k}$ commutes with $T$, the geodesic $\gamma_{1}$ is also an axis of $T_{\bar{\gamma}}^{k}$ :

$$
T_{\bar{\gamma}}^{k}\left(\gamma_{1}\right)=T_{\bar{\gamma}}^{k} T(\bar{\gamma})=T T_{\bar{\gamma}}^{k}(\bar{\gamma})=T(\bar{\gamma})=\gamma_{1} .
$$

Therefore, $T_{\bar{\gamma}}^{k}$ is a power of $T_{\gamma_{1}}$. But $T_{\bar{\gamma}}$ and $T_{\gamma_{1}}$ have the same critical displacement along $\gamma_{1}$, and hence, since $\pi_{1}(M)$ is torsion free, we must have that $T_{\bar{\gamma}}=T_{\gamma_{1}}$. Finally, recall that $T_{\gamma_{1}}$ is obtained by conjugating $T_{\bar{\gamma}}$ :

$$
T_{\gamma_{1}}=T T_{\bar{\gamma}} T^{-1}
$$

This follows from the fact that the map $T T_{\bar{\gamma}} T^{-1}$ preserves the geodesic $\gamma_{1}$, where its critical displacement is $l$ (as it is easy to check). Thus, the actions of $T_{\bar{\gamma}}$ and $T T_{\bar{\gamma}} T^{-1}$ coincide along $\gamma_{1}$, and being $\pi_{1}(M)$ torsion free the two maps must coincide. This finishes the proof of the Claim and the Lemma.

A straightforward consequence of Lemma 6.2 and Lemma 6.1 is the following:

Lemma 6.3. Let $\gamma \subset M$ be a closed geodesic, $\bar{\gamma}$ a lift of $\gamma, T_{\bar{\gamma}}$ the generator of the group of covering isometries preserving $\bar{\gamma}$. Then a lift $\beta$ of $\gamma$ is an axis of $T_{\bar{\gamma}}$ if and only if $\beta$ is asymptotic to $\bar{\gamma}$.

Proof. Indeed, if $\beta$ is asymptotic to $\bar{\gamma}$ it is actually bi-asymptotic to $\bar{\gamma}$. So we have a covering isometry $F$ such that $\beta=F(\bar{\gamma})$ which commutes with $T_{\bar{\gamma}}$ by Lemma 6.2 . But once $F$ commutes with $T_{\bar{\gamma}}$ we have by Lemma 6.1 , (3) that $F$ takes axes of $T_{\bar{\gamma}}$ into axes of $T_{\bar{\gamma}}$. Hence, $\beta$ must be an axis of $T_{\bar{\gamma}}$ as well. Moreover, if $\beta$ is an axis of $T_{\bar{\gamma}}$ it was already shown in the proof of Lemma 6.2.

An application of Lemma 6.1 is Lemma 4.8 which was stated in Subsection 4.4 .

Lemma 6.4. Let $(M, g)$ be compact without conjugate points. Let $\gamma_{\theta}, \gamma_{\sigma}$ be two different, bi-asymptotic lifts in $\tilde{M}$ of a closed geodesic $\gamma \in M$. Then they are Busemann asymptotes of each other. 
Proof. Let $T$ be a covering isometry such that $\gamma_{\theta}$ is an axis of $T$. By Lemma 6.1 item 2, the geodesic $\gamma_{\sigma}$ is also an axis of $T$ and the displacement of $T$ restricted to both $\gamma_{\theta}$ and $\gamma_{\sigma}$ attains its minimum value $l=\operatorname{Per}(\gamma)$, the minimum period of $\gamma$. Let us assume that $\gamma_{\sigma}(0) \in H_{\theta}(0)$. Since $T$ is an isometry we have that $T\left(H_{\theta}(t)\right)=H_{\theta}(t+l)$ for every $t \in \mathbb{R}$. So $T\left(\gamma_{\sigma}(0)\right) \in H_{\theta}(l)$ and hence, by Lemma 5.1, the distance

$$
d\left(\gamma_{\sigma}(0), T\left(\gamma_{\sigma}(0)\right)\right)=d_{T}\left(\gamma_{\sigma}(0)\right)
$$

coincides with the distance between $H_{\theta}(0)$ and $H_{\theta}(l)$ that is precisely $l$. Since the distance between two horospheres $H_{\theta}(t)$ and $H_{\theta}(s)$ is attained at the geodesics of the Busemann flow $\psi_{t}^{\theta}$, the geodesic $\gamma_{\sigma}$ must be an orbit of the Busemann flow $\psi_{t}^{\theta}$ thus proving the lemma.

This basic result will be used in the last sections of the survey.

There is a classical, rich theory about the links between the geometry of $\tilde{M}$ and algebraic properties of $\pi_{1}(M)$ for nonpositive curvature manifolds. Each time we refer to a subgroup of $\pi_{1}(M)$ we really mean a subgroup of $\pi_{1}(M, p)$ for some $p \in M$. We shall adopt this convention from now on to shorten notation. Just to give some examples of results: normal subgroups of $\pi_{1}(M)$ give rise to the existence of an isometry between $\tilde{M}$ and a product manifold; the well known Bieberbach theorems of groups of isometries in Euclidean space have corresponding versions for nonpositive curvature. Good references for the above subject are the books of Wolf [102], CheegerEbin [23], and Ballmann-Gromov-Schröeder [8]. If we drop the assumption on the curvature sign very few is known about the relationships between the algebraic structure of $\pi_{1}(M)$ and rigidity properties of $\tilde{M}$. The following result due to Croke-Schröeder [26] is perhaps the best we can say about manifolds without conjugate points concerning the subject.

Theorem 6.1. Let $(M, g)$ be a compact, manifold without conjugate points. Then every nilpotent subgroup of $\pi_{1}(M)$ is abelian. If in addition we suppose that $M$ is analytic, then $\pi_{1}(M)$ is Bieberbach: every solvable subgroup of $\pi_{1}(M)$ contains a finite index abelian subgroup.

We shall come back to this theorem and its proof in the forthcoming subsections, it contains some ideas about the geometry of subgroups of $\pi_{1}(M)$ that will be relevant for the main results of the survey.

\section{Fundamental group in negative curvature, Preissmann's theorem and Gromov hyper- bolic groups}

In this subsection we focus on some remarkable properties of $\pi_{1}(M)$ when $(M, g)$ has negative curvature. One of the first fundamental links between 
algebraic properties of $\pi_{1}(M)$ and negative curvature geometry is the famous Preissmann's theorem [76].

Theorem 6.2. Let $(M, g)$ be a compact manifold whose sectional curvatures are strictly negative. Then every abelian subgroup of $\pi_{1}(M)$ is infinite cyclic.

One way of proving this theorem is the following. Suppose that $G \subset$ $\pi_{1}(M)$ is an abelian, subgroup. Let $T, S$ be two nontrivial elements of $G$, and let us assume without loss of generality that $T$ has the smallest displacement of $G$. Since $T S=S T$ by Lemma $6.1 S$ and $T$ permute the axes of each other. Moreover, is a good elementary exercise to show that $S^{n}$ preserves an axis of $T$ if and only if $S$ is in the cyclic group generated by $T$, since $\pi_{1}(M)$ has no torsion. So let $\gamma$ be an axis of $T$, then $S(\gamma)$ is also an axis of $T$ which remains at bounded distance from $T$, because the axes of $T$ are lifts of closed geodesics in the same homotopy class. And since the distance between geodesics in negative curvature spaces is strictly convex, such distance has to be zero. Thus, $S(\gamma)=\gamma$ and therefore, $S$ is in the group generated by $T$.

Preissmann's theorem is one of the first results relating the geometry of negative curvature and algebraic properties of $\pi_{1}(M)$. Of course, the theory of manifolds of negative curvature experienced an extraordinary development since the times of Preissmann. The so-called geometric group theory introduced by Gromov and Thurston is the most broad, clear framework to understand the rich interaction between geometry and algebra in spaces of negative curvature.

Let us give a brief account of definitions and some results of the theory of Gromov hyperbolic spaces. Our main references are [46] and [27]. We recall the definition of Gromov hyperbolic spaces given in the Introduction. A metric space $(X, d)$ is called a geodesic space if every pair of points can be joined by a geodesic segment.

Definition 6.1. A complete geodesic space $(X, d)$ is called $\delta$-hyperbolic (or simply hyperbolic) if every geodesic triangle is $\delta$-thin, i.e., every point of a given side of the triangle is at distance at most $\delta$ from the union of the other two sides.

$\delta$-hyperbolic spaces are often called Gromov hyperbolic. This notion contains the essential characterization of geodesic triangles in the usual hyperbolic space, and is based in the so-called CAT-comparison theory (from Caratheodory, Alexandrov, Toponogov). Observe that the definition of $\delta$-thin spaces is very general, we do not need a Riemannian metric for the definition. This allows to study a wide range of metric spaces from the point of view of hyperbolic geometry, including trees, groups, and polygonal spaces. 
Of course, the hyperbolic plane and the universal covering of compact manifolds of negative curvature are Gromov hyperbolic. Compact manifolds whose geodesic flow is Anosov have Gromov hyperbolic universal coverings. This fact is a combination of a result due to Klingenberg [58] who shows that universal coverings of compact, Anosov manifolds have the so-called visibility property (see Section 3 of this chapter)- and Theorem 6.8 in this chapter. If $(M, g)$ is a compact surface without conjugate points of genus greater than one, then $(\tilde{M}, \tilde{g})$ is a Gromov hyperbolic space too by the work of Morse. Surfaces without conjugate points of higher genus are good examples of how the notion of Gromov hyperbolicity captures the global, coarse geometry of spaces.

The fundamental group of such manifolds can be regarded as a Gromov hyperbolic space as well, by means of the word metric. Namely, given a symmetric set of generators of $\pi_{1}(M)$, we define the length of an element $T$ of $\pi_{1}(M)$ as the number of generators in the shortest string representing $T$ as a product of such generators. The word distance $w(T, S)$ from $T$ to $S$ is the word length of $T^{-1} S$. The group $\pi_{1}(M)$ acts by isometries of the metric space $\left(\pi_{1}(M), w\right)$ by left multiplication. We can even turn this discrete metric space into a continuous metric space, by constructing the Cayley graph $\Gamma \pi_{1}(M)$ of the group $\pi_{1}(M)$ : we put a segment of length one between any two elements of $\pi_{1}(M)$ whose distance in the word metric is one, and extend the word metric to a continuous metric in this graph.

If we change the set of symmetric generators, the word metrics are quasiisometric.

Definition 6.2. A map $f: X \longrightarrow Y$ between two geodesic spaces $(X, d)$, $(Y, h)$ is a quasi-isometry if there exist positive constants $L, B$ and a map $h: Y \longrightarrow X$ satisfying the following conditions:

$$
\begin{aligned}
& \text { 1. } d(f(p), f(q)) \leq L d(p, q)+B \forall p, q \in X \\
& \text { 2. } d(h(p), h(q)) \leq L d(p, q)+B \forall p, q \in Y \\
& \text { 3. } d(f(h(p)), p) \leq B \forall p \in Y \\
& \text { 4. } d(h(f(p)), p) \leq B \forall p \in X
\end{aligned}
$$

The spaces $X$ and $Y$ are called quasi-isometric and we shall refer to the image $f(X)$ either as a quasi-isometric immersion of $X$ or as a quasiisometric copy of $X$. Notice that the map $h$ above can be regarded as a sort of quasi-inverse of $f$. The notion of quasi-isometry allows us to identify $\pi_{1}(M)$ with a sort of discrete analogous of $\tilde{M}$.

Theorem 6.3. Let $(M, g)$ be a compact Riemannian manifold. Then $(\tilde{M}, \tilde{g})$ and $\left(\pi_{1}(M), w\right)$ are quasi-isometric. 
Another definition for quasi-geodesic, equivalent to the previous one, is the following:

Definition 6.3. Let $(X, d)$ be a metric space. A rectifiable curve $C$ parametrized by arc length $c:[a, b] \longrightarrow X$ is called $A, B$-quasi-geodesic if the metric spaces $(c, \hat{d})$ and $([a, b],||$.$) are A, B$-quasi-isometric, where $\hat{d}$ is the metric induced by $d$ on the curve $c$ and $|$.$| is the metric given$ by the absolute value in the interval $[a, b]$.

So a quasi-geodesic is quasi-isometric to a geodesic. The theory of Gromov hyperbolic spaces is very close to the theory of negatively curved manifolds, although Gromov hyperbolic spaces are much more general spaces (exponential growth of balls, compactification and ideal boundaries, etc, etc). A very interesting area of geometric group theory deals with the so-called geometric properties of groups: these are properties of groups which are preserved by quasi-isometries. We won't enter into details in this matter, the reader can look at the references. We shall mention some fundamental results for the sake of completeness.

Lemma 6.5. Let $G$ be a finitely generated group. Then any two word metrics in $G$ give two quasi-isometric metric structures on $G$.

Theorem 6.4. Gromov hyperbolicity is a geometric property.

The following result proved by Gromov [46] extends Morse's work for compact surfaces without conjugate points.

Theorem 6.5. Let $(X, d)$ be a Gromov hyperbolic space. Given $A, B>0$, there exists $D=D(A, B)>0$ such that every $A, B$ quasigeodesic is contained in the tubular neighborhood of radius $D$ of a geodesic.

The following statement proved by Bonk [16] classifies Gromov hyperbolic spaces in terms of tracing of quasi-geodesics by true geodesics of the space.

Theorem 6.6. Let $(X, d)$ be a complete geodesic space. If for every given $A, B>0$ there exists $D=D(A, B)>0$ such that every $A, B$ quasi-geodesic is contained in the D-tubular neighborhood of a geodesic, then $(X, d)$ is a Gromov hyperbolic space.

The above results are very interesting counterparts of the stability features of Anosov dynamics. Theorem 6.5 is analogous to the $C^{1}$ stability of Anosov dynamics, but it is stronger in the sense that Theorem 6.5 holds for every quasi-geodesic of the Gromov hyperbolic space, not just for quasi-geodesics arising from perturbations of the system. Observe that, since Gromov hyperbolicity is equivalent to the tracing of quasi-geodesics by geodesics, and Gromov hyperbolicity is a geometric property, then the tracing of quasi-geodesics by geodesics is a geometric property. Moreover, 
since $\pi_{1}(M)$ endowed with the word metric and $(\tilde{M}, \tilde{g})$ are quasi-isometric, then the Gromov hyperbolicity of one of them implies the Gromov hyperbolicity of the other one.

Theorem 6.6 can be compared to the stability conjecture proved by Mañé [65], which characterizes hyperbolic dynamics by structural stability. However, Theorem 6.6 seems to be weaker than the stability conjecture because it requires that all quasi-geodesics have to be shadowed by geodesics to grant the Gromov hyperbolicity of the space.

Both theorems are concerned with the relationship between shadowing (or tracing) of quasi-geodesics by geodesics and hyperbolicity. Such results inspire some of the main results that we shall discuss next.

\section{Preissmann property implies Gromov hyperbolicity?}

This natural question is in fact a hard problem closely related with Thurston's geometrization conjecture. Some partial answers are known for manifolds with nonpositive curvature, and these results will be crucial for the proofs of the main theorems of the survey. The first important step towards the study of this problem is the following statement due to Eberlein-O'Neil [35].

Theorem 6.7. Let $(M, g)$ be a compact manifold of nonpositive curvature. If $(\tilde{M}, \tilde{g})$ is not a visibility manifold then there exists a totally geodesic, flat plane in $\tilde{M}$.

The manifold $(\tilde{M}, \tilde{g})$ is a (uniform) visibility manifold if given $\epsilon>0$ there exists $r>0$ such that for every $p, x, y \in \tilde{M}$, if the distance between $p$ and the geodesic $[x, y]$ is greater than $r$, then the angle at $p$ formed by the geodesics $[p, x],[p, y]$ is less than $\epsilon$. The universal covering of compact manifolds of negative curvature are visibility manifolds. Eberlein-O'Neil showed that once a compact manifold $M$ without conjugate points admits a visibility structure, then the universal covering of every metric in $M$ without conjugate points is a visibility manifold. The notion of visibility is actually almost equivalent to Gromov hyperbolicity, from [84], [91] we have,

Theorem 6.8. Let $(M, g)$ be compact without conjugate points. Then $(\tilde{M}, \tilde{g})$ is a visibility manifold if and only if geodesic rays diverge in $\tilde{M}$ and $M$ is Gromov hyperbolic.

Proof. We just make a sketch of the proof pointing out the role of the divergence of geodesic rays in the argument. 
We show first that the Gromov hyperbolicity and divergence of geodesic rays in $\tilde{M}$ imply visibility. Let $\delta>0$ be such that every geodesic triangle in $\tilde{M}$ is $\delta$-thin. Let us denote by $[x, y]$ the geodesic joining $x, y$. It is easy to see that there exists $D=D(\delta)$ such that in every geodesic triangle $\left[x_{0}, x_{1}\right] \cup\left[x_{1}, x_{2}\right] \cup\left[x_{2}, x_{0}\right]$ there exists three points $y_{i} \in\left[x_{i}, x_{i+1}\right]$ (indices taken mod. 3) with $d\left(y_{i}, y_{i+1}\right) \leq D$.

Let us suppose that the distance between $x_{0}$ and every point in $\left[x_{1}, x_{2}\right]$ is greater than $L>0$. By the triangle inequality, we have that

$$
\inf \left\{d\left(x_{0}, y_{0}\right), d\left(x_{0}, y_{2}\right)\right\} \geq L-D \text {. }
$$

So we have two geodesic rays $\gamma_{0}, \gamma_{2}$ starting at $x_{0}=\gamma_{0}(0)=\gamma_{2}(0)$, namely, the geodesic rays containing the geodesic segments $\left[x_{0}, x_{1}\right],\left[x_{0}, x_{2}\right]$ respectively, having points $y_{0} \in \gamma_{0}, y_{2} \in \gamma_{2}$, such that

1. $d\left(\gamma_{i}(0), y_{i}\right) \geq L-D$, for $i=0,2$.

2. $d\left(y_{0}, y_{2}\right) \leq D$.

It is not hard to see that the compactness of $M$ implies the uniform divergence of rays in $\tilde{M}$ (see [91] for instance). So given $\epsilon>0$, there exists $T>$ such that if $L-D \geq T$, the angle formed by $\gamma_{0}$ and $\gamma_{2}$ at $x_{0}$ is less than $\epsilon$, which implies visibility as we wished.

To show that visibility implies divergence of geodesic rays and Gromov hyperbolicity, let us observe that the divergence of geodesic rays is just straightforward from the visibility property. The proof of the fact that geodesic triangles are $\delta$-thin for some $\delta>0$ goes by contradiction. Suppose that geodesic triangles are not $\delta$-thin for any $\delta>0$. Then given $n \in \mathbb{N}$, there exists a geodesic triangle $\left[x_{n}, y_{n}\right] \cup\left[y_{n}, z_{n}\right] \cup\left[z_{n}, x_{n}\right]$, and a point $t_{n} \in\left[x_{n}, y_{n}\right]$ such that

$$
\inf \left\{d\left(p, t_{n}\right), p \in\left[y_{n}, z_{n}\right] \cup\left[z_{n}, x_{n}\right]\right\} \geq n \text {. }
$$

Consider the geodesic triangles

$$
\left[t_{n}, x_{n}\right] \cup\left[x_{n}, z_{n}\right] \cup\left[z_{n}, t_{n}\right]
$$

and

$$
\left[t_{n}, y_{n}\right] \cup\left[y_{n}, z_{n}\right] \cup\left[z_{n}, t_{n}\right]
$$

By the visibility assumption, the angle $\alpha_{n}$ formed by the geodesics $\left[t_{n}, z_{n}\right]$, $\left[t_{n}, x_{n}\right]$ at the point $t_{n}$ must go to zero as $n \rightarrow+\infty$. The same holds for the angle $\theta_{n}$ formed by the geodesics $\left[t_{n}, z_{n}\right],\left[t_{n}, y_{n}\right]$ at the point $t_{n}$. On the other hand, notice that the sum $\alpha_{n}+\theta_{n}$ is equal to $\pi$, which is clearly a contradiction. 
From Theorem 6.8, Theorem 6.5 and Theorem 6.6 we can deduce in fact that if $(M, g)$ is compact without conjugate points and $(\tilde{M}, \tilde{g})$ is a visibility manifold, then every metric in $M$ without conjugate points has the same property. Eberlein showed that visibility manifolds enjoy many important properties of manifolds of negative curvature, like the existence of compactification for $\tilde{M}$, the existence of the cone topology, the existence of an extension of the action of $\pi_{1}(M)$ to the boundary of the compactification, and the verification of many properties of the hyperbolic topological dynamics of this action. Later, Gromov in [46] proved the same results (and many more) for Gromov hyperbolic spaces using more abstract, general arguments. We could say that nowadays, the theory of visibility manifolds is included in the theory of Gromov hyperbolic spaces.

Next, Bangert and Schröeder in [11] showed the following amazing result:

Theorem 6.9. Let $(M, g)$ be a compact analytic Riemannian manifold with nonpositive curvature. If $\tilde{M}$ contains a flat, totally geodesic isometric embedding of $\mathbb{R}^{k}$, for some $k \geq 2$ then $M$ contains an immersed, totally geodesic flat torus.

Of course, if $M$ contains a flat, totally geodesic flat torus then the fundamental group of such torus is a non cyclic, abelian subgroup of $\pi_{1}(M)$. So combining Theorems $6.3,6.7,6.8$, and Theorem 6.9 we get

Theorem 6.10. Let $(M, g)$ be a compact analytic manifold with nonpositive curvature. Then $(\tilde{M}, \tilde{g})$ is Gromov hyperbolic if and only if $\pi_{1}(M)$ is Preissmann: every abelian subgroup of $\pi_{1}(M)$ is infinite cyclic.

So the question posed in the beginning of the section is completely answered in the category of compact analytic manifolds with nonpositive curvature. There is a generalization of Eberlein's flat plane theorem for quasi-convex manifolds without conjugate points [87]:

Theorem 6.11. Let $(M, g)$ be a compact manifold without conjugate points such that $(\tilde{M}, \tilde{g})$ is quasi-convex. Then $(\tilde{M}, \tilde{g})$ is not Gromov hyperbolic if and only if there exists a quasi-isometric immersion of the Euclidean plane in $(\tilde{M}, \tilde{g})$.

Of course, the techniques employed to study the analytic nonpositive curvature case do not apply to the quasi-convex, $C^{\infty}$ case. However, in the light of the recent work of Perelman about the Poincaré conjecture and Thurston's geometrization conjecture, it seems that Theorem 6.10 would hold for three dimensional, irreducible compact manifolds without conjugate points. 


\section{Expansive dynamics, weak stability and Gro- mov hyperbolicity}

We would like to finish the chapter with an application of the notion of Gromov hyperbolicity to study the geometry of $\tilde{M}$ in the case of expansive geodesic flows. The main result of the section is the following [84]:

Theorem 6.12. Let $(M, g)$ be a compact manifold without conjugate points whose geodesic flow is expansive. Then $(\tilde{M}, \tilde{g})$ is Gromov hyperbolic.

We have already showed in Chapter 5 that expansive geodesic flows of compact manifolds without conjugate points enjoy many of the properties of the topological dynamics of Anosov systems. The above theorem shows that the analogy between such systems extends to the global geometry of the universal covering of the manifold. The interesting point of Theorem 6.12 is that the expansiveness assumption does not involve Jacobi fields or sectional curvatures. Just the expansiveness of the geodesic flow is enough to grant the Gromov hyperbolicity of $\tilde{M}$.

We give next an outline of the proof of Theorem 6.12. We would like to remind that by Corollary 5.1 , the universal covering $(\tilde{M}, \tilde{g})$ is quasi-convex. The key lemma of the proof is the following.

Proposition 6.1. Let $(M, g)$ be a compact manifold without conjugate points such that $(\tilde{M}, \tilde{g})$ is quasi-convex. If $(\tilde{M}, \tilde{g})$ is not Gromov hyperbolic, the following is true: given $n \in \mathbb{N}$, there exists a pair of geodesics $\gamma_{n}, \beta_{n}$ in $\tilde{M}$ such that

1. $\gamma_{n}$ and $\beta_{n}$ are bi-asymptotic.

2. For every $t \in \mathbb{R}$ we have that

$$
\inf _{s \in \mathbb{R}} d\left(\gamma_{n}(t), \beta_{n}(s)\right) \geq n .
$$

3. For every $s \in \mathbb{R}$ we have

$$
\inf _{t \in \mathbb{R}} d\left(\gamma_{n}(t), \beta_{n}(s)\right) \geq n
$$

Proposition 6.1 tells us that the failure of the Gromov hyperbolicity implies the existence for every $n \in \mathbb{N}$ of bi-asymptotic geodesics in $\tilde{M}$ which are far away from each other at a distance of at least $n$. But Lemma 5.2 such geodesics cannot exist if the geodesic flow is expansive.

Notice that Proposition 6.1 gives a proof of the flat plane theorem of Eberlein (Theorem 6.7). Indeed, by the geometry of nonpositive curvature, such geodesics bound a flat strip whose width is at least $n$. Passing to a 
convergent subsequence of strips $S_{n}$ of width at least $n$ (this subsequence exists because of the co-compact isometric action of $\left.\pi_{1}(M)\right)$ we get a flat plane in $\tilde{M}$.

The proof of Proposition 6.1 follows from a careful analysis of the shapes of non-thin geodesic triangles in $\tilde{M}$. The main idea of the proof of the Lemma is the following: if we consider grids of geodesic segments joining the endpoints of appropriate partitions of different sides of a fat geodesic triangle in $\tilde{M}$, we would expect to get from the grid a fat geodesic quadrilateral. So what we can show is that given $n \in \mathbb{N}$, there exists $L(n)>0$ such that if we have a sequence of increasingly fatter geodesic triangles, we get a sequence of increasingly larger geodesic quadrilaterals whose width is bounded above by $L(n)$ and bounded below by $n$.

Let us be more precise. We shall denote by $[a, b]$ the geodesic joining $a, b$ in $\tilde{M}$. Suppose that $(\tilde{M}, \tilde{g})$ is not Gromov hyperbolic. Then there exists a sequence of geodesic triangles $\Delta\left(p_{n}, q_{n}, r_{n}\right)$ in $\tilde{M}$, whose vertices are the points $p_{n}, q_{n}, r_{n}$, with the following property:

There exists $x_{n} \in\left[p_{n}, q_{n}\right]$ such that

$$
\inf _{y \in\left[p_{n}, r_{n}\right] \cup\left[q_{n}, r_{n}\right]} d\left(x_{n}, y\right) \rightarrow+\infty,
$$

when $n \rightarrow+\infty$. Let us introduce the following definition which describes the asymptotic shape of certain sequences of non-hyperbolic triangles.

Definition 6.4. Let $\triangle_{n}$ be a sequence of geodesic triangles with vertices $p_{n}, q_{n}, r_{n}$. We say that the sequence of geodesic quadrilaterals

$$
\square_{n}=\left[a_{n}^{1}, b_{n}^{1}\right] \cup\left[b_{n}^{1}, b_{n}^{2}\right] \cup\left[a_{n}^{2}, b_{n}^{2}\right] \cup\left[a_{n}^{1}, a_{n}^{2}\right],
$$

where $a_{n}^{1}, b_{n}^{1} \in\left[p_{n}, q_{n}\right], a_{n}^{2}, b_{n}^{2} \in\left[p_{n}, r_{n}\right]$, is a thin up-to-scale sequence of quadrilaterals if they satisfy the following conditions:

1. $d\left(a_{n}^{1}, p_{n}\right)<d\left(b_{n}^{1}, p_{n}\right)$ for every $n$,

2. $d\left(a_{n}^{1},\left[p_{n}, r_{n}\right]\right)=d\left(a_{n}^{1}, a_{n}^{2}\right)$, and $d\left(b_{n}^{1},\left[p_{n}, r_{n}\right]\right)=d\left(b_{n}^{1}, b_{n}^{2}\right)$ for every $n$,

3. There exists $A>0$ such that $A d\left(b_{n}^{1}, b_{n}^{2}\right) \leq d\left(a_{n}^{1}, a_{n}^{2}\right)$ for every $n$,

4. $d\left(a_{n}^{1}, a_{n}^{2}\right) \rightarrow+\infty$ with $n \rightarrow+\infty$,

5. $d\left(a_{n}^{1}, b_{n}^{1}\right) \rightarrow+\infty$ with $n \rightarrow+\infty$,

6. $\frac{d\left(b_{n}^{1}, b_{n}^{2}\right)}{d\left(a_{n}^{1}, b_{n}^{1}\right)} \rightarrow 0$ when $n \rightarrow+\infty$. 
By the definition it is not difficult to show that there exists $n_{0}>0$ such that $d\left(a_{n}^{2}, p_{n}\right)<d\left(b_{n}^{2}, p_{n}\right)$ for every $n>n_{0}$, and hence the quasi-convexity of $(\tilde{M}, \tilde{g})$ yields that

$$
d\left(a_{n}^{1}, a_{n}^{2}\right) \leq K d\left(b_{n}^{1}, b_{n}^{2}\right)+C,
$$

for every $n>n_{0}$ where $K, C$ are the quasi-convexity constants of $(\tilde{M}, \tilde{g})$. So $d\left(b_{n}^{2}, p_{n}\right)$ tends to $+\infty$ as well, as a consequence of the definition. The term thin up-to-scale is suggestive: if we think of the geodesics $\left[a^{1}, a^{2}\right],\left[b_{n}^{1}, b_{n}^{2}\right]$ as the "vertical" sides of the quadrilateral $\square_{n}$, item (6) in the definition tells us that the hight of $\square_{n}$ compared with the length of its base $\left[a_{n}^{1}, b_{n}^{1}\right]$ is very small. So re-scaling with respect to the length of $\left[a_{n}^{1}, b_{n}^{1}\right]$, the quadrilaterals $\square_{n}$ look like very thin rectangles.

The next result shows that the existence of thin up-to-scale sequences of quadrilaterals implies Proposition 6.1.

Lemma 6.6. Let $(M, g)$ be a compact manifold without conjugate points such that $(\tilde{M}, \tilde{g})$ is $K, C$-quasi-convex. Assume that there exists a sequence of geodesic triangles $\triangle_{n}$, with vertices $p_{n}, q_{n}, r_{n}$, containing the vertices $a_{n}^{1}, a_{n}^{2}, b_{n}^{1}, b_{n}^{2}$ of a sequence of thin up-to-scale geodesic quadrilaterals $\square_{n}$. Then given $m \in \mathbb{N}$, there exists a pair of geodesics $\gamma_{m}, \beta_{m}$ in $\tilde{M}$ such that

1. $\gamma_{m}$ and $\beta_{m}$ are bi-asymptotic.

2. For every $t \in \mathbb{R}$ we have that

$$
\inf _{s \in \mathbb{R}} d\left(\gamma_{m}(t), \beta_{m}(s)\right) \geq m
$$

3. For every $s \in \mathbb{R}$ we have

$$
\inf _{t \in \mathbb{R}} d\left(\gamma_{m}(t), \beta_{m}(s)\right) \geq m
$$

Proof. Let $D>0$ be an arbitrary positive number, and let us make partitions

$$
\begin{aligned}
{\left[a_{n}^{1}, a_{n}^{2}\right] } & =\cup_{i=0}^{j_{n}-1}\left[x_{i}^{n}, x_{i+1}^{n}\right], \\
{\left[b_{n}^{1}, b_{n}^{2}\right] } & =\cup_{i=0}^{j_{n}-1}\left[y_{i}^{n}, y_{i+1}^{n}\right],
\end{aligned}
$$

with the following properties:

1. $x_{0}^{n}=a_{n}^{1}, x_{j_{n}}^{n}=a_{n}^{2}$, and $y_{0}^{n}=b_{n}^{1}, y_{j_{n}}^{n}=b_{n}^{2}$.

2. $d\left(y_{i}^{n}, y_{i+1}^{n}\right)=D$ for every $i \in\left[0, j_{n}-1\right], d\left(y_{j_{n}-1}^{n}, y_{j_{n}}^{n}\right) \leq D$.

3. $d\left(x_{i}^{n}, x_{i+1}^{n}\right)=d\left(x_{i+1}^{n}, x_{i+2}^{n}\right)$ for every $i \in\left[0, j_{n}-2\right]$. 
Since there exists $n_{0}>0$ such that for $n>n_{0}$ the length of $\left[a_{n}^{1}, a_{n}^{2}\right]$ satisfies

$$
d\left(a_{n}^{1}, a_{n}^{2}\right) \leq K d\left(b_{n}^{1}, b_{n}^{2}\right)+C,
$$

the assumptions on the partitions imply that there exists $n_{1}>0$ such that for every $n>n_{1}$, and $j_{n} \geq 2$ we get

$$
A D \leq d\left(x_{i}^{n}, x_{i+1}^{n}\right) \leq K D+2,
$$

for every $i \in\left[0, j_{n}\right]$. Let us consider the geodesics

$$
\sigma_{i}^{n}=\left[x_{i}^{n}, y_{i}^{n}\right]
$$

for every $i \in\left[0, j_{n}\right]$. Notice that $\sigma_{0}^{n}=\left[a_{n}^{1}, b_{n}^{1}\right]$, and $\sigma_{j_{n}}^{n}=\left[a_{n}^{2}, b_{n}^{2}\right]$.

Claim: There exists $\lambda=\lambda(A, K, C, D)>0$ for which there exist $i_{n} \in$ $\left[0, j_{n}\right]$, and a point $w_{n} \in \sigma_{i_{n}}^{n}$, such that

1. $d\left(w_{n}, \sigma_{i_{n}+1}^{n}\right) \geq \lambda D$,

2. $\lim _{n \rightarrow+\infty} d\left(w_{n}, x_{i_{n}}^{n}\right)=\infty$ and $\lim _{n \rightarrow+\infty} d\left(w_{n}, y_{i_{n}}^{n}\right)=\infty$.

Otherwise, for every $\lambda>0$ there would exist $l>0$ such that for every $i \in\left[0, j_{n}\right]$, and $p \in \sigma_{i}^{n}$ with $d\left(p, x_{i}^{n}\right) \geq l, d\left(p, y_{i}^{n}\right) \geq l$, we would get

$$
d\left(p, \sigma_{i+1}^{n}\right) \leq \lambda D .
$$

This yields, by the triangular inequality, that there exist points $z_{n} \in \sigma_{0}^{n}$, $s_{n} \in \sigma_{j_{n}}^{n}$, such that $d\left(z_{n}, s_{n}\right) \leq j_{n} \lambda D$. Since we are assuming that $\tilde{M}$ is $K, C$-quasi-convex, we have that

$$
d\left(a_{n}^{1}, a_{n}^{2}\right) \leq K d\left(z_{n}, s_{n}\right)+C \leq K j_{n} \lambda D+C,
$$

since $\sigma_{0}^{n} \subset\left[p_{n}, b_{n}^{1}\right], \sigma_{j_{n}}^{n} \subset\left[p_{n}, b_{n}^{2}\right]$. Combining this with the assumptions on the partitions we get

$$
A\left(j_{n} D\right) \leq A d\left(b_{n}^{1}, b_{n}^{2}\right) \leq d\left(a_{n}^{1}, a_{n}^{2}\right) \leq K j_{n} \lambda D+C .
$$

Dividing by $K D j_{n}$ we obtain

$$
\frac{A D}{K} \leq \lambda+\epsilon_{n}
$$

where $\epsilon_{n} \rightarrow 0$ if $n \rightarrow+\infty$. This yields that for $n$ big enough, $\lambda$ must be greater than $\frac{A D}{2 K}$, which clearly leads to a contradiction.

The claim gives us a sequence of pairs of geodesics $\sigma_{i_{n}}^{n}, \sigma_{i_{n}+1}^{n}$ of the form

$$
\sigma_{i_{n}}^{n}=\left[p_{n}, y_{i_{n}}^{n}\right], \sigma_{i_{n}+1}^{n}=\left[p_{n}, y_{i_{n}+1}^{n}\right] \text {, }
$$

that satisfy 
1. The Hausdorff distance from $\sigma_{i_{n}}^{n}$ to $\sigma_{i_{n}+1}^{n}$ is less than $K \sup \{D, K D+$ $2\}+C$ by quasi-convexity,

2. The minimum distance from a point in $\sigma_{i_{n}}^{n}$ to $\sigma_{i_{n}+1}^{n}$ is at least $\lambda D$; as well as the minimum distance from a point of $\sigma_{i_{n}+1}^{n}$ to $\sigma_{i_{n}}^{n}$.

3. The conditions of Claim 1 hold.

Letting $n \rightarrow+\infty$, and taking convergent subsequences of appropriate iterates of $\sigma_{i_{n}}^{n}, \sigma_{i_{n}+1}^{n}$ by covering isometries, we get the pair of bi-asymptotic geodesics which are separated by a distance of at least $\lambda D$. Since $D$ is arbitrary, we can take $m \leq \lambda D$ to get the geodesics required in Proposition 6.1.

The next asymptotic configuration of non-hyperbolic geodesic triangles is in some sense the complement of the thin up-to-scale configuration.

Definition 6.5. We say that a sequence of geodesic triangles $\triangle_{n}, n \in \mathbb{N}$, is $L$-fat for $L>0$ with respect to its vertices $p_{n}, q_{n}, r_{n}$ if

1. inf $f_{s \in\left[0, d\left(q_{n}, r_{n}\right)\right.}\left\{d\left(p_{n},\left[q_{n}, r_{n}\right](s)\right\} \geq L \sup \left\{d\left(p_{n}, r_{n}\right), d\left(q_{n}, r_{n}\right), d\left(p_{n}, q_{n}\right\}\right.\right.$, where $\left[q_{n}, r_{n}\right](s)$ is an arc length parametrization of $\left[q_{n}, r_{n}\right]$ with $\left[q_{n}, r_{n}\right](0)=q_{n}$.

2. For every $t \in\left[0, d\left(p_{n}, q_{n}\right)\right]$ we have

$$
d\left(\left[p_{n}, q_{n}\right](t),\left[p_{n}, r_{n}\right]\right) \geq L t,
$$

where $\left[p_{n}, q_{n}\right](t)$ is an arc length parametrization of $\left[p_{n}, q_{n}\right]$ with $\left[p_{n}, q_{n}\right](0)=p_{n}$.

$L$-fat sequences of geodesic triangles have sequences of pairs of sides whose distance grows at least linearly, while in the case of thin up-to-scale sequences there were sequences of sides looking like very thin rectangles up to re-scaling. Observe that the notion of $L$-fatness is relative to a certain choice of the vertices of the triangles.

Notice as well that the definition of $L$-fat triangles implies that the lengths of the sides of the triangles in the sequence are comparable. More precisely, let $d_{n}^{1} \leq d_{n}^{2} \leq d_{n}^{3}$, be the lengths of the sides $l_{n}^{1}, l_{n}^{2}, l_{n}^{3}$, of $\triangle_{n}$ ordered by non-decreasing length. Then $d_{n}^{1}, d_{n}^{2}$ are the distances from the vertices of $l_{n}^{3}$ to the common vertex of $l_{n}^{1} \cap l_{n}^{2}$. So if the common vertex is $p_{n}$ in the definition of $L$-fatness we get from item (1) that

1. $L d_{n}^{3} \leq d_{n}^{1} \leq d_{n}^{3}$,

2. $L d_{n}^{3} \leq d_{n}^{2} \leq d_{n}^{3}$,

3. $L d_{n}^{2} \leq d_{n}^{1} \leq \frac{1}{L} d_{n}^{2}$, 
4. $L d_{n}^{1} \leq d_{n}^{2} \leq \frac{1}{L} d_{n}^{1}$.

Otherwise, if $p_{n}$ is in $l_{n}^{3} \cap l_{n}^{1}$ for instance we get from item (2) that

$$
L d_{n}^{3} \leq d^{2}
$$

and from item (1) we get

$$
L d_{n}^{3} \leq d^{1} .
$$

So we get again the same above set of inequalities comparing all the lengths of the sides of $\triangle_{n}$. When $p_{n}$ is in $l_{n}^{3} \cap l_{n}^{2}$ the argument is just analogous.

The following result yields the proof of Proposition 6.1 for fat sequences too.

Lemma 6.7. Let $(M, g)$ be a compact manifold without conjugate points such that $(\tilde{M}, \tilde{g})$ is $K, C$-quasi-convex. Assume that there exists a sequence of geodesic triangles $\triangle_{n}$, with vertices $p_{n}, q_{n}, r_{n}$, that is $L$-fat. Then given $m \in \mathbb{N}$, there exists a pair of geodesics $\gamma_{m}, \beta_{m}$ in $\tilde{M}$ such that

1. $\gamma_{m}$ and $\beta_{m}$ are bi-asymptotic.

2. For every $t \in \mathbb{R}$ we have that

$$
\inf _{s \in \mathbb{R}} d\left(\gamma_{m}(t), \beta_{m}(s)\right) \geq m .
$$

3. For every $s \in \mathbb{R}$ we have

$$
\inf _{t \in \mathbb{R}} d\left(\gamma_{m}(t), \beta_{m}(s)\right) \geq m
$$

Proof. We just sketch the proof since it is quite similar to the proof of Lemma 6.6. The difference in this case is the grid of geodesics constructed through partitions of the sides of the sequence of triangles. So we describe the construction of the grid and leave to the reader the details of the proof of the Lemma.

Given $D>0$ we define partitions

$$
\begin{aligned}
& {\left[p_{n}, q_{n}\right]=\cup_{i=0}^{j_{n}-1}\left[x_{i}^{n}, x_{i+1}^{n}\right],} \\
& {\left[p_{n}, r_{n}\right]=\cup_{i=0}^{j_{n}-1}\left[y_{i}^{n}, y_{i+1}^{n}\right],}
\end{aligned}
$$

with the following properties:

1. $x_{0}^{n}=p_{n}=y_{0}^{n}, x_{j_{n}}^{n}=q_{n}$, and $y_{j_{n}}^{n}=r_{n}$.

2. $d\left(y_{i}^{n}, y_{i+1}^{n}\right)=D$ for every $i \in\left[0, j_{n}-1\right], d\left(y_{j_{n}-1}^{n}, y_{j_{n}}^{n}\right) \leq D$.

3. $d\left(x_{i}^{n}, x_{i+1}^{n}\right)=d\left(x_{i+1}^{n}, x_{i+2}^{n}\right)$ for every $i \in\left[0, j_{n}-2\right]$. 
Observe that since $d\left(p_{n}, r_{n}\right) \geq d\left(p_{n}, q_{n}\right)$ we get that $d\left(x_{i}^{n}, x_{i+1}^{n}\right) \leq D$ for every $i \in\left[0, j_{n}-1\right]$. As in the previous lemma, let $\sigma_{i}^{n}=\left[x_{i}^{n}, y_{i}^{n}\right]$.

Claim: There exists $\eta=\eta(L, K, C, D)>0$ for which there exist $i_{n} \in$ $\left[0, j_{n}\right]$, and a point $w_{n} \in \sigma_{i_{n}}^{n}$, such that

1. $d\left(w_{n}, \sigma_{i_{n}+1}^{n}\right) \geq \eta D$,

2. $\lim _{n \rightarrow+\infty} d\left(w_{n}, x_{i_{n}}^{n}\right)=\infty$ and $\lim _{n \rightarrow+\infty} d\left(w_{n}, y_{i_{n}}^{n}\right)=\infty$.

The proof follows the same lines of the proof of the Claim in Lemma 6.6 and we leave it to the reader. Once we have the sequence of pairs of geodesics $\sigma_{i_{n}}^{n}, \sigma_{i_{n}+1}^{n}$ we can obtain after a limiting process (like in the proof of Lemma 6.6) the pair of bi-asymptotic geodesics required by Lemma 6.7.

Finally, the proof of Proposition 6.1 follows from Lemma 6.6, Lemma 6.7, and the next result that is proved in [84].

Lemma 6.8. Let $(M, g)$ be a compact manifold without conjugate points such that $(\tilde{M}, \tilde{g})$ is $K, C$-quasi-convex. Assume that $(\tilde{M}, \tilde{g})$ is not Gromov hyperbolic. Then one of the following assertions hold:

1. Either there exists $L>0$ and $L$-fat sequence of geodesic triangles in $\tilde{M}$,

2. Or there exists a sequence of geodesic triangles containing the vertices of a thin up-to-scale sequence of geodesic quadrilaterals.

The main idea of the proof of Lemma 6.8 is quite natural. First of all, the lack of Gromov hyperbolicity implies the existence of a sequence $\triangle_{n}$ of large geodesic triangles whose sides get more and more apart as $n \rightarrow+\infty$. The point is in what way such sides get apart from each other. Lemma 6.8 says that this happens roughly in two different ways: either the sequence $\triangle_{n}$ has a sequence of sides whose distances diverge at least $L$-linearly for some constant $L>0$ (namely, $\triangle_{n}$ is $L$-fat for some $L>0$ ), or there are sequences of pairs of sides in the sequence $\triangle_{n}$ which look like thin ends up to re-scaling as $n \rightarrow+\infty$. In this case we can show that there exists a sequence of thin up-to-scale quadrilaterals whose vertices belong to the triangles $\triangle_{n}$. We won't write the complete proof of Lemma 6.8 , for the details we refer to [84].

Some further comments to conclude the chapter. First of all, the ideas of the proof of Theorem 6.12, specially Lemma 6.1, are the starting point for the proof of Theorem 6.11. With a certain (nontrivial) amount of work, one can show that distant bi-asymptotic geodesics give rise to subsets of $\tilde{M}$ which are quasi-isometric to flat strips. Then, if $(\tilde{M}, \tilde{g})$ is not Gromov 
hyperbolic, a limit process using sequences of such quasi-strips shows the existence of a quasi-isometric copy of a flat plane.

Finally, Theorem 5.2 implies that the expansiveness of the geodesic flow of a compact manifold $(M, g)$ without conjugate points yields the shadowing property. Theorem 6.12 combined with Theorem 6.5 show that such flows have the property of shadowing of quasi-geodesics of $(\tilde{M}, \tilde{g})$. This last property says that for any metric $(M, h)$, the globally minimizing geodesics of $(\tilde{M}, \tilde{h})$ are traced by geodesics of $(\tilde{M}, \tilde{g})$. So $(M, h)$ might not be a perturbation of $(M, g)$ and nevertheless, the globally minimizing geodesics in $(\tilde{M}, \tilde{h})$ behave roughly like the geodesics of $(\tilde{M}, \tilde{g})$. The shadowing of quasi-geodesics is a strong property in geometric group theory, it characterizes Gromov hyperbolic spaces. We shall show in the forthcoming chapters that the shadowing of quasi-geodesics which are just geodesics of perturbations of $(M, g)$ still implies in certain manifolds that the universal covering is a Gromov hyperbolic space. How much can one weaken stability assumptions on the geodesic flow and still have hyperbolicity in the large in $\tilde{M}$ ? 


\section{Chapter 7}

\section{Weak hyperbolicity of the geodesic flow and global hyperbolic geometry in nonpositive curvature}

The purpose of Chapters 7 and 8 is to give an overview, including very recent results, of a theory which involves weak stability properties of the geodesic flow in manifolds without conjugate points, the global geometry of the universal covering, and geometric group theory. This theory gathers all the tools we have presented until now and illustrates very well the interaction between topological dynamics, geometry and group theory. The subject of the last two chapters has been developed essentially in four articles: [88], [89], [90], [92]. Let us start with a new definition.

Definition 7.1. We say that a smooth flow $\phi_{t}: N \longrightarrow N$ satisfies the $\epsilon-C^{k}$-shadowing property for some $\epsilon>0$ if there exists a $C^{k}$ neighborhood $V$ of $\phi_{t}$ such that for every $\psi_{t} \in V$ there is a continuous map $f: N \longrightarrow N$ such that for every $p \in N$ there exists a continuous surjective map $r: \mathbb{R} \longrightarrow$ $\mathbb{R}$ with $r(0)=0$ and

$$
d\left(\phi_{t}(f(p)), \psi_{r(t)}(p)\right) \leq \epsilon,
$$

for every $t \in \mathbb{R}$.

The $\epsilon$ - $C^{k}$-shadowing property is clearly inspired in the shadowing property of pseudo-orbits defined in Chapter 2 (Definition 2.13). However, this notion involves the $\epsilon$-approximation of orbits of perturbations $\psi_{t}$ of the flow $\phi_{t}$ by orbits of $\phi_{t}$. So from the point of view of the classical pseudo-orbit tracing property of Chapter 2, Definition 7.1 requires the tracing of those pseudo-orbits of $\phi_{t}$ which are as well orbits of perturbations of $\phi_{t}$. Therefore, the $\epsilon-C^{k}$-shadowing property of a flow $\phi_{t}$ is weaker than the tracing of pseudo-orbits of $\phi_{t}$. 
Definition 7.1 resembles the notion of topological stability, in the following sense: the possibility of $\epsilon$-shadowing every orbit of a perturbation of a system by true orbits of the system might suggest the existence of a correspondence between the perturbed orbits and the orbits of the system. In fact, the pseudo-orbit tracing property is one of the main ingredients of the proof of the structural stability of Axiom A diffeomorphisms and Anosov systems: the shadowing of pseudo-orbits implies the structural stability in these cases as we showed in Chapter 2.

Lemma 2.3 in Chapter 2 together with the results in Chapter 5 yield that expansive geodesic flows in compact manifolds without conjugate points have the shadowing property of pseudo-orbits, like expansive homeomorphisms of compact surfaces [62]. So expansive geodesic flows in compact manifolds without conjugate points have the $\epsilon$ - $C^{0}$-shadowing property for an appropriate $\epsilon$ (depending on the expansiveness constant) according to Definition 7.1. And the results in Chapter 2 imply that the pseudo-orbit tracing property of such expansive systems yields their topological stability (in general, expansive systems are not structurally stable). However, there are $C^{1}$ generic families of diffeomorphisms which are not hyperbolic where the $C^{1}$ pseudo-orbit tracing property does not hold [17], [1]; so the shadowing property in expansive, non-hyperbolic systems is exceptional in some sense. It is interesting to remark that the tracing of pseudo-orbits in expansive systems implies that the semi-conjugacies with nearby systems are homotopic to the identity. So in many respects the pseudo-orbit tracing property might be considered stronger than the assumption of topological stability, and in some families of two dimensional, $C^{1}$ generic systems the $C^{1}$ shadowing property actually implies structural stability [93], [94]. In more general contexts there is no clear relationship between shadowing and stability.

On the other hand, the $\epsilon$ - $C^{k}$-shadowing property has a strong flavor of the shadowing of quasi-geodesics by true geodesics occurring in Gromov hyperbolic spaces. Let us recall that this property characterizes Gromov hyperbolic spaces according to a theorem due to Bonk [16] as we already observed in Chapter 6. So both the classical theory of hyperbolic topological dynamics and the theory of Gromov hyperbolic spaces, suggest that the $\epsilon$ - $C^{k}$-shadowing property, as well as the topological stability, should imply that $(M, g)$ might be hyperbolic in some sense. Based on the above considerations, we formulate a conjecture that could be viewed as a sort of "topological stability conjecture" for geodesic flows of manifolds without conjugate points.

\section{Conjecture (?):}

Let $(M, g)$ be a compact, smooth Riemannian manifold without conjugate points such that the geodesic flow enjoys either the $\epsilon-C^{k}$-shadowing 
property for some $k \geq 0$ and $\epsilon>0$, or the $C^{k}$ topological stability for some $k \geq 1$. Then the universal covering of $M$ endowed with the pullback of the metric $g$ is a Gromov hyperbolic space.

We would like to remark that the ideas used by Bonk in [16] cannot be applied to our problem. Bonk's result implies that $(M, g)$ is Gromov hyperbolic provided that for every $A, B>0$ there exists $C=C(A, B)$ such that every $A, B$-quasi-geodesic is in the $C$-tubular neighborhood of a geodesic in $(M, g)$. Our hypothesis about the class of curves in the space that are shadowed by geodesics is more restrictive: we just assume that geodesics of perturbations of $(M, g)$ can be shadowed by geodesics, and the collection of quasi-geodesics of $(M, g)$ might be much larger than the collection of geodesics of perturbed metrics.

We shall discuss in this chapter the nonpositive curvature case where we have a much better understanding of the global geometry of $\tilde{M}$. The main results of the chapter show that the conjecture is true in certain manifolds with nonpositive curvature. Our main reference is [88].

Theorem 7.1. Let $M$ be a compact, analytic Riemannian manifold of nonpositive curvature. If the geodesic flow of $M$ is $C^{k}$ topologically stable for some $k>0$ then the universal covering $\tilde{M}$ of $M$ is a Gromov hyperbolic space.

Theorem 7.2. Let $M$ be a compact, analytic Riemannian manifold of nonpositive curvature. Let $\rho>0$ be the injectivity radius of $M$. Then there exists a constant $C>0$ such that if the geodesic flow of $M$ satisfies the $C \rho-C^{k}$-shadowing property for some $k>0$ then the universal covering $\tilde{M}$ of $M$ is Gromov hyperbolic.

The analyticity of the metric assumed in the above two theorems is very important for their proofs. Indeed, if we drop the analyticity hypothesis we get a much weaker result: both assumptions, the $\epsilon-C^{k}$-shadowing property and the topological stability grant that the fundamental group has the Preissmann property. So the solution of the conjecture leads naturally to the problem discussed in Chapter 6 concerning the relationship between the Preissmann property and Gromov hyperbolicity. This issue is closely related with the solution of Thurston's geometrization conjecture in the case of three dimensional manifolds. We shall comment in more detail this subject at the end of the survey.

In fact, Theorems 7.1 and 7.2 hold assuming $C^{\infty}$ topological stability or $\epsilon$-shadowing instead of $C^{k}$. We shall subdivide the proofs of the theorems in many steps. 


\section{Homoclinic geodesics in the two torus and instability}

We follow the notations of Chapters 1 and 6 concerning the fundamental group and covering isometries. A covering isometry $T$ is naturally identified with an element of one of the point base fundamental groups $\pi_{1}(M, p)$, $p \in M$. So we shall write the statement $T \in \pi_{1}(M)$ as a convention to stress this identification.

There is a key idea that is common to the proof of Theorems 7.1 and 7.2: the instability of the geodesic flow of a flat metric in the two torus produced by the existence of homoclinic geodesics in arbitrarily small perturbations of such metrics. Given a flat metric structure in the two torus, $\left(T^{2}, g\right)$, and a closed geodesic $\gamma_{\theta}$, we find $C^{\infty}$ perturbations $\bar{g}$ of $g$ such that

1. the geodesic $\gamma_{\theta}$ is a geodesic of $\left(T^{2}, \bar{g}\right)$,

2. the geodesic $\gamma_{\theta}$ is a waist in its homotopy class, i.e., the $\bar{g}$-length of every closed loop freely homotopic to $\gamma_{\theta}$ is strictly bigger than the $\bar{g}$-length of $\gamma_{\theta}$.

Under this conditions, it was proved by Morse [69] that there exists a minimizing $\bar{g}$-geodesic $\beta \subset\left(T^{2}, \bar{g}\right)$ that is homoclinic to $\gamma_{\theta}$ : the $\alpha$ and $\omega$ limits of $\beta$ coincide with $\gamma_{\theta}$. Moreover, if we take two consecutive lifts $\gamma_{1}$, $\gamma_{2}$ of $\gamma_{\theta}$ in the universal covering of $T^{2}$ (i.e., there is no other lift of $\gamma_{\theta}$ in the strip bounded by $\gamma_{1}$ and $\gamma_{2}$ ), there is a lift $\tilde{\beta}$ of $\beta$ contained in the strip bounded by $\gamma_{1}$ and $\gamma_{2}$ such that

$$
\lim _{t \rightarrow+\infty} d\left(\tilde{\beta}(t), \gamma_{1}\right)=0,
$$

and

$$
\lim _{t \rightarrow-\infty} d\left(\tilde{\beta}(t), \gamma_{2}\right)=0 .
$$

The existence of the homoclinic geodesic $\beta$ yields the proof of the conjecture for the two torus, a fact that we might naturally expect but that is very important for the proof of the conjecture in nonpositive curvature.

Lemma 7.1. The geodesic flow of a flat metric in $T^{2}$ is not $C^{k}$ topologically stable for any $k \geq 0$.

Proof. Assume that the geodesic flow of $\left(T^{2}, g\right)$ is topologically stable in some $C^{k}$ topology. Let $\gamma$ be the closed geodesic of $\left(T^{2}, g\right)$ of minimum length. Let $(M, \bar{g})$ be a $C^{k}$ perturbation of $(M, g)$ of the type described above, that turns $\gamma$ into a waist in its homotopy class. We remark that $\bar{g}$ can be constructed arbitrarily close to $g$ in the $C^{\infty}$ topology. Let $\beta$ be the homoclinic $\bar{g}$-geodesic above, and let $\gamma_{1}, \gamma_{2}$ be two consecutive lifts of $\gamma$ in $\tilde{T}^{2}=\mathbb{R}^{2}$, bounding a strip in $\mathbb{R}^{2}$ where there is no other lift of a 
closed $\bar{g}$-geodesic homotopic to $\gamma$. Let $\tilde{\beta}$ the lift of $\beta$ in this strip with the asymptotic properties stated above. Consider the semi-equivalence

$$
f:\left(T_{1} T^{2}, \bar{g}\right) \longrightarrow\left(T_{1} T^{2}, g\right)
$$

provided by the topological stability of the geodesic flow of $\left(T^{2}, g\right)$ (to simplify notation, we denote by $g$ and $\bar{g}$ the Sasaki metrics associated to $g$ and $\bar{g}$ respectively). Let us also denote by $\gamma, \beta \tilde{\beta}, \gamma_{1}, \gamma_{2}$ the orbits of the corresponding geodesic flows associated to these geodesics.

Claim: $f(\beta)=f(\gamma)$.

Indeed, $f(\gamma)$ is an orbit of the geodesic flow of the flat metric $\left(T^{2}, g\right)$, that is closed because $\gamma$ is closed. By continuity of $f$, the geodesic $f(\beta)$ must approach $f(\gamma)$ in the future and in the past. But the geodesic $f(\gamma)$ corresponds to the projection of a straight line of $\mathbb{R}^{2}$ in $T^{2}$, and hence the only geodesic of $\left(T^{2}, g\right)$ that approaches $f(\gamma)$ is $f(\gamma)$ itself, thus proving the claim.

Let $l>0$ be the minimum period of $\gamma$, and let us consider the closed loops $c_{n}$ in $T^{2}$ formed in the following way: join the point $\gamma_{2}(-n l)$ to a close point in $\tilde{\beta}$, say $\tilde{\beta}\left(t_{n}\right)$, with a geodesic segment $\left[\gamma_{2}(-n l), \tilde{\beta}\left(t_{n}\right)\right]$; join the point $\gamma_{1}(n l)$ to a close point in $\tilde{\beta}$, say $\tilde{\beta}\left(s_{n}\right)$, with a geodesic segment $\left[\gamma_{1}(n l), \tilde{\beta}\left(s_{n}\right)\right]$; consider the curve

$$
C_{n}=\left[\gamma_{2}(-n l), \tilde{\beta}\left(t_{n}\right)\right] \cup \tilde{\beta}\left(\left[t_{n}, s_{n}\right]\right) \cup\left[\gamma_{1}(n l), \tilde{\beta}\left(s_{n}\right)\right],
$$

and let $c_{n}=\Pi\left(C_{n}\right)$, where $\Pi: \mathbb{R}^{2} \longrightarrow T^{2}$ is the covering map. Observe that, as $n \rightarrow+\infty$, the lengths of the geodesic segments $\left[\gamma_{2}(-n l), \tilde{\beta}\left(t_{n}\right)\right]$ and $\left[\gamma_{2}(n l), \tilde{\beta}\left(s_{n}\right)\right]$ tend to zero. So we have that

$$
\lim _{n \rightarrow+\infty} t_{n}=-\infty, \lim _{n \rightarrow+\infty} s_{n}=\infty .
$$

Moreover, since $f$ is continuous, and $f(\beta)=f(\gamma)$, the images $f\left(\beta\left(t_{n}\right)\right)$ and $f\left(\beta\left(s_{n}\right)\right)$ are close to $f(\gamma(0))$. So we can suppose without loss of generality that $f\left(\beta\left(t_{n}\right)\right)=f\left(\beta\left(s_{n}\right)\right)$. Since $f$ is a surjective semi-equivalence, $f$ is surjective on the closed orbits of the geodesic flow and then, the induced linear map

$$
f_{*}: H_{1}\left(T^{2}, \mathbb{Z}\right) \longrightarrow H_{1}\left(T^{2}, \mathbb{Z}\right)
$$

in the first homology group is surjective. A linear, surjective transformation of $\mathbb{R}^{m}$ onto itself must be an isomorphism. Denote by $[c]$ the homology class of a closed curve in $T_{1} T^{2}$. Notice that the homology class of $c_{n}$ is

$$
\left[c_{n}\right]=2 n[\gamma]+x,
$$

where $x \in H_{1}\left(T^{2}, \mathbb{Z}\right)$ is a nontrivial homology class. Hence, we have that

$$
f_{*}\left(\left[c_{n}\right]\right)=2 n f_{*}([\gamma])+f_{*}(x) .
$$


However, taking $n$ large, $f\left(c_{n}\right)$ is a closed curved composed by $f(\gamma)$ together with two small closed curves homological to zero. Therefore,

$$
f_{*}\left(\left[c_{n}\right]\right)=2 n f_{*}([\gamma])+f_{*}(x)=2 n f_{*}([\gamma]),
$$

which implies that $f_{*}(x)=0$ contradicting the fact that $f_{*}$ is an isomorphism.

The fact that the geodesic flow is not structurally stable is much easier to show, because a geodesic flow in $T^{2}$ with a homoclinic orbit cannot be equivalent to the geodesic flow of a flat torus. The next lemma concerns the shadowing property. It can be interpreted in the following way: if the geodesic flow of a flat two torus has the $\epsilon-C^{k}$ shadowing property for some $\epsilon>0$, then the diameter of the torus has to be of the order of $\epsilon$. Therefore, if $\epsilon$ is small enough, the geodesic flow of a given flat torus cannot have the $\epsilon$ shadowing property.

Lemma 7.2. Let $\left(T^{2}, g\right)$ be a flat metric in the torus, whose injectivity radius is $\rho$. Then the geodesic flow of $\left(T^{2}, g\right)$ does not have the $\frac{\rho}{5}-C^{k}$ shadowing property for any $k \geq 0$.

Proof. First of all, let us explain the choice of the constant $\frac{\rho}{5}$. The injectivity radius is one half of the length of the shortest closed geodesic of the manifold. It is not difficult to show (see for instance [88], Lemma 1.6) that we can choose generators $v_{1}, v_{2}$ of the fundamental group of $\left(T^{2}, g\right)$ such that

1. The shortest closed geodesic of $\left(T^{2}, g\right)$ has homotopy class $v_{1}$,

2. the length of a closed geodesic with homotopy class $v_{2}$ is the shortest among the lengths of closed geodesics excluding the loops with homotopy class $v_{1}$,

3 . identifying $v_{1}, v_{2}$ with vectors in $\mathbb{R}^{2}$, the angle between $v_{1}$ and $v_{2}$, $\angle\left(v_{1}, v_{2}\right)$, satisfies $\frac{\pi}{3} \leq \angle\left(v_{1}, v_{2}\right) \leq \frac{2 \pi}{3}$.

A fundamental domain of $T^{2}$ taking $v_{1}, v_{2}$ as generators of the fundamental group is a parallelogram whose sides have lengths $l_{1}=\rho, l_{2}$, where $l_{i}$ is the length of a closed geodesic with homotopy class $v_{i}$. The estimate for the angle formed by $v_{1}, v_{2}$ implies that there exists $C>0$ such that the distance between the sides of this parallelogram is bounded above by $C \rho$. Elementary trigonometry shows that $\frac{C}{2}>\frac{1}{5}$, and that is why the number $\frac{\rho}{5}$ appears in the statement.

Assume, by contradiction, that the geodesic flow $\phi_{t}$ of $\left(T^{2}, g\right)$ satisfies the $\epsilon-C^{k}$-shadowing property for some $\epsilon \leq \frac{\rho}{5}$. Let $\alpha_{1}, \alpha_{2}$ be closed geodesics of $\left(T^{2}, g\right)$ whose homotopy classes are respectively $v_{1}, v_{2}$.

Claim: The distance between two consecutive lifts of $\alpha_{1}$ in the universal covering is less than $2 \epsilon$. 
In fact, let us consider a $C^{\infty}$ perturbation $\left(T^{2}, g_{1}\right)$ of $\left(T^{2}, g\right)$ such that $\alpha_{1}$ is strictly minimizing in its homotopy class with respect to the $g_{1}$-length. Let $\tilde{\beta}$ be the geodesic of $\left(\mathbb{R}^{2}, g_{1}\right)$ mentioned above, such that $\beta=\pi(\tilde{\beta})$ is homoclinic to $\alpha_{1}$ and such that $\tilde{\beta}$ is asymptotic to two different, consecutive lifts $\tilde{\alpha}_{1}, \tilde{\alpha}_{2}$ of $\alpha_{1}, \alpha_{2}$. Since $\beta$ is $\epsilon$-shadowed by some geodesic $\gamma$ in $\left(T^{2}, g\right)$, by the choice of $\epsilon$ we have that the lift $\tilde{\beta}$ is $\epsilon$-shadowed in $\mathbb{R}^{2}$ by a lift $\tilde{\gamma}$ of $\gamma$. But a lift of $\gamma$ is a straight line, and hence $\tilde{\gamma}$ has to be parallel to both $\tilde{\alpha}_{1}$ and $\tilde{\alpha}_{2}$. Since $\tilde{\beta}$ is contained in the strip bounded by $\tilde{\alpha}_{1}$ and $\tilde{\alpha}_{2}$, it is not difficult to see that the width of this strip has to be at most $2 \epsilon$ as we wished to show.

But now, the choice of $\epsilon$ implies that $2 \epsilon<2 \frac{C}{2} \rho=C \rho$, and this yields that the distance between $\tilde{\alpha}_{1}$ and $\tilde{\alpha}_{2}$ is less than $C \rho$, contradicting the choice of $C$. The contradiction arose from the assumption of $\epsilon-C^{k}$ shadowing, therefore the geodesic flow of $\left(T^{2}, g\right)$ cannot have the this property for any $\epsilon \leq \frac{\rho}{5}$ as claimed.

\section{The closing lemma for flat planes in ana- lytic manifolds with nonpositive curvature and the proof of the conjecture in this case}

The second important ingredient of the proof of the conjecture for nonpositively curved, analytic manifolds is Theorem 6.9 due to Bangert and Schröeder, which essentially says that isometric embeddings of $\mathbb{R}^{k}$ for $k \geq 2$ in $\tilde{M}$ give immersed, totally geodesic tori in $M$.

The third key idea to prove the conjecture is Eberlein's characterization (Theorem 6.7) of nonpositive curvature manifolds whose universal coverings are not Gromov hyperbolic: the universal covering contains flat planes.

So the idea to prove the conjecture for analytic compact manifolds of nonpositive curvature goes by contradiction. Suppose that the universal covering of $(M, g)$ is not Gromov hyperbolic. Then $\tilde{M}$ has a flat plane and hence there exists an immersed flat, totally geodesic two torus $T$ in $(M, g)$. Now, it is shown in [88], Section 3, that there exist arbitrarily small, $C^{\infty}$ perturbations $(M, \bar{g})$ of the metric $g$ which keep $T$ totally geodesic and such that $(T, \bar{g})$ has all the features mentioned in the previous subsection: a given closed geodesic in $(T, g)$ becomes a waist in $(T, \bar{g})$ and therefore all the results of Subsection 2.1 hold for the geodesic flow of $(T, \bar{g})$. The convexity of nonpositive curvature geometry allows to extend the instability results obtained for $(T, \bar{g})$ to $(M, \bar{g})[88]$, thus finishing the proof of the conjecture in this case. 
A brief summary of the argument would be the following: the geodesic flow of a flat metric in the two torus has neither the topological stability property nor the shadowing property. The same conclusion can be extended to compact analytic manifolds of nonpositive curvature because we can reduce the problem to deal with a flat torus. The Bangert-Schröeder closing lemma of flats in analytic manifolds is crucial to the argument. Therefore, the extension of this technique to the $C^{\infty}$ case is not possible in general.

\section{Sharpening the shadowing result by means of Aubry-Mather theory}

The variational arguments employed in the previous subsection have a flavor of Aubry-Mather theory. The description given by Morse and Hedlund [49] of globally minimizing geodesics in surfaces has many natural counterparts in the dynamics of minimizing orbits of measure preserving twist maps of the annulus, first studied by Birkhoff [15].

Recall that a measure preserving map of the closed annulus $F: S^{1} \times$ $[0,1] \longrightarrow S^{1} \times[0,1], F(t, s)=(\bar{t}, \bar{s})$, is called a monotone twist map if

1. $F$ preserves the boundary of $S^{1} \times[0,1]$, i.e., the restrictions $\left.F\right|_{S^{1} \times\{0\}}$, $\left.F\right|_{S^{1} \times\{1\}}$ are diffeomorphisms

$$
\begin{aligned}
& \left.F\right|_{S^{1} \times\{0\}}: S^{1} \times\{0\} \longrightarrow S^{1} \times\{0\}, \\
& \left.F\right|_{S^{1} \times\{1\}}: S^{1} \times\{0\} \longrightarrow S^{1} \times\{1\} .
\end{aligned}
$$

2. $F$ is a diffeomorphism in $S^{1} \times(0,1)$ which satisfies

$$
\frac{\partial \bar{t}}{\partial s} \neq 0
$$

for every point in $S^{1} \times(0,1)$.

The name twist map comes from the fact that the images of the vertical lines $\{p\} \times[0,1]$ by $F$ are always transversal to the vertical lines and can be expressed as graphs of monotone functions defined in connected subsets of $S^{1}$. There is a natural variational principle associated to monotone twist maps whose minima are certain orbits of the dynamics satisfying very special properties, the so-called Aubry-Mather sets (for definitions and a clear, simple exposition of the theory we refer to [9]).

It is well known that the geodesic flow of a flat two torus can be represented, locally, by a measure preserving twist map of the annulus. Indeed, any region in the unit tangent bundle bounded by two invariant tori with different rotation numbers can be represented by a measure preserving, 
monotone twist map in a certain annulus, the so-called Birkhoff map which we recall briefly in the next paragraph.

Given a Riemannian metric $\left(T^{2}, h\right)$ in $T^{2}$, and a simple closed geodesic $\beta$, the Birkhoff map $P_{\beta}$ associated to $\beta$ is the first return map of geodesics intersecting $\beta$ transversally. Namely, if $\gamma$ is a geodesic in $\left(T^{2}, h\right)$ with $\gamma(0)=\beta(s)$, and $\gamma^{\prime}(0)$ is transversal to $\beta^{\prime}(0)$, then $P_{\beta}(\gamma)=\gamma(r)=\beta(\bar{s})$ is the next intersection of $\gamma$ with $\beta$. If we assume that such next intersection always exists, the Birkhoff map induces a map

$$
\begin{aligned}
T_{\beta}: S^{1} \times(0,2 \pi) & \longrightarrow S^{1} \times(0,2 \pi), \\
T_{\beta}(s, t) & =(\bar{s}, \bar{t}),
\end{aligned}
$$

where $S^{1}=R / Z,(s, t)$ represents the geodesic $\gamma_{\theta}=\gamma_{\theta(s, t)}$ with initial conditions $\gamma_{\theta}(0)=\beta\left(s l_{h}(\beta)\right), t=\cos ^{-1} h\left(\gamma_{\theta}^{\prime}(0), \beta^{\prime}\left(s l_{h}(\beta)\right)\right)$. So the coordinate $t$ is a branch of the angle with respect to $\beta$. Analogously, $(\bar{s}, \bar{t})$ represents the first return of $\gamma_{\theta}$ to $\beta$, i.e., $P_{\beta}\left(\gamma_{\theta}\right)=\gamma_{\theta}(r)=\beta\left(\bar{s} l_{h}(\beta)\right)$, and $\bar{t}=\cos ^{-1} h\left(\gamma_{\theta}^{\prime}(r), \beta^{\prime}\left(\bar{s} l_{h}(\beta)\right)\right)$. This map might not be defined everywhere, but in the case of flat metrics in $T^{2}$ (and sufficiently small perturbations of such metrics), it is possible to represent by a suitable Birkhoff map the whole dynamics of an invariant set of the geodesic flow bounded by two invariant tori with different rotation numbers.

The representation of the geodesic flow by a twist map allows us to establish a correspondence between objects and properties of geodesic flows and twist maps: geodesics correspond to orbits of a twist map; asymptotic behavior (according to Hedlund's work [49]) of geodesics is a natural counterpart of the rotation number of orbits of a twist map; invariant tori of the geodesic flow correspond to invariant curves of the twist map, globally minimizing geodesics (Definition 4.1 in Chapter 4) are counterparts of Mather sets. Of course, the twist map associated to any region bounded by two invariant tori of a flat metric is totally integrable: the domain of the twist map is foliated by invariant curves.

The local representation of the geodesic flow by twist maps also holds for geodesic flows in the two torus of metrics which are sufficiently close to a flat metric in the $C^{4}$ topology. This is due to KAM theory, which grants the persistence of many invariant tori in $C^{4}$ perturbations of a flat metric. So the motivation for the results in [90] is to use this link between geodesic flows in the torus and the powerful Mather theory about minimizing orbits of twist maps to improve the results in the previous subsection.

Recall that the main tool used to produce instability was the existence of homoclinic geodesics after perturbing the geodesic flow. The asymptotic 
behavior of the homoclinic geodesics is determined by the asymptotic behavior of a closed geodesic. By the representation of the geodesic flow by twist maps, heteroclinic geodesics correspond to the so-called connecting orbits of a twist map (i.e., orbits whose $\alpha$ and $\omega$ limits are Mather sets with different rotation numbers). Given two closed, minimizing geodesics $\gamma_{1}$ and $\gamma_{2}$ with different asymptotic directions in the two torus, a geodesic $\beta$ is heteroclinic to $\gamma_{1}, \gamma_{2}$ if $\beta$ is $\alpha$-asymptotic to $\gamma_{1}$ and $\omega$-asymptotic to $\gamma_{2}$. Heteroclinic behavior of geodesics in the torus is much far away from Euclidean geometry than homoclinic behavior. So we might guess that the creation of heteroclinic behavior (that is more "chaotic" in some sense) by perturbations of a flat metric in the torus would improve the results obtained in Section 2. Let us consider the following new definition of shadowing of geodesics.

Definition 7.2. Let $(M, g)$ be a complete, smooth Riemannian manifold. We say that the geodesic flow of $(M, g)$ satisfies the $C^{k}$-shadowing property if there exists a $C^{k}$ neighborhood $V$ of $(M, g)$ such that every metric $\bar{g} \in$ $V$ has the following property: given a geodesic $\gamma$ in $(\tilde{M}, \bar{g})$, there exist a geodesic $\beta$ in $(\tilde{M}, g)$ within a finite Hausdorff distance from $\gamma$.

Notice that the $\epsilon$ in the definition of the shadowing property given in Definition 7.1 is not involved in Definition 7.2. The new shadowing property just requires that perturbed geodesics are shadowed by geodesics of the initial metric, regardless of the distance to the "shadow". The main results in [90] concern this stronger notion of shadowing.

Theorem 7.3. Let $(M, g)$ be a compact Riemannian manifold with nonpositive curvature. If $(M, g)$ satisfies the $C^{4}$ shadowing property then $(M, g)$ contains no immersed, flat, totally geodesic tori.

Observe that Theorem 7.3 is inspired by the results of Section 4, where the main idea of the proofs of Theorems 7.1 and 7.2 was that the presence of flat tori in the manifold implied instability. Applying Theorem 7.3, the ideas of the Section and the theory of rank one manifolds we get:

Theorem 7.4. Let $(M, g)$ be a compact manifold with nonpositive curvature. Assume that $(M, g)$ has the $C^{k}$ shadowing property for some $k>0$. Then

1. The rank of $M$ is one, the Pesin set of the geodesic flow has positive Lebesgue measure, and in particular, the metric entropy of the geodesic flow with respect to the Lebesgue measure is positive.

2. If $(M, g)$ is analytic, the universal covering $(\tilde{M}, g)$ endowed with the pullback of $g$ is a Gromov hyperbolic space.

Indeed, item (1) of Theorem 7.4 follows from the fact that flat, totally geodesic tori are dense in compact, higher rank manifolds of nonpositive 
curvature [7], [5], and hence by Theorem 7.3 such manifolds cannot have the shadowing property. Rank one manifolds have many hyperbolic-like ergodic properties like a positive Lebesque measure Pesin set which coincides with an open and dense subset of $T_{1} M$ almost everywhere (see [5], [61] for instance, or [82] for a similar result for manifolds with bounded asymptote).

Item (2) combines the ideas of Section 2 with Theorem 7.3: the BangertSchröeder closing lemma of flats (Theorem 6.9) holds in compact, analytic manifolds of nonpositive curvature and therefore the shadowing property prevents the existence of flats in the universal covering. Then, Eberlein's theorem (Theorem 6.7) implies that the universal covering is a Gromov hyperbolic space.

The main issue of the section is Theorem 7.3. The crucial idea of the proof of this theorem is the existence of a sort of complicated "almost" heteroclinic behavior in perturbations of a flat metric in the two torus. The proof of this fact relies in the following result due to Mather [67]:

Theorem 7.5. Let $F: S^{1} \times \mathbb{R} \longrightarrow S^{1} \times \mathbb{R}$ be a monotone twist map, and let $B$ be a Birkhoff region of instability. Let $\Gamma_{-}, \Gamma_{+}$be the invariant curves in the boundary of $B$, with rotation numbers $\rho_{-}<\rho_{+}$, and let $M_{F, \omega}$ be the Mather set of $F$ with rotation number $\omega \in\left(\rho_{-}, \rho_{+}\right)$. Consider for each $i \in \mathbb{Z}$ a real number $\omega_{i} \in\left[\rho_{-}, \rho_{+}\right]$and a positive number $\epsilon_{i}$. Then there exists an orbit $\left\{F^{k}(x)\right\}$ in $B$ and an increasing bi-infinite sequence $\left\{j_{i}\right\}$ of integers such that $d\left(F^{j_{i}}(x), M_{F, \omega_{i}}\right)<\epsilon_{i}$.

Recall that a Birkhoff region of instability $B$ of a twist map $F: S^{1} \times$ $R \longrightarrow S^{1} \times R$ is an open annulus bounded by two homotopically nontrivial, invariant curves of $F$ where there are no other invariant curves of $F$. Mather's theorem implies that in a Birkhoff region of instability, given any sequence of rotation numbers $\omega_{i}$ in the rotation interval $\left[\rho_{-}, \rho_{+}\right]$of the monotone twist map in the Birkhoff region, there exists an orbit $O(x)$ of the twist map with a large piece $O_{i}$ "shadowed" by the Mather set whose rotation number is $\omega_{i}$, for every $i \in \mathbb{Z}$. This is not exactly what we meant by heteroclinic behavior, because the orbit $O(x)$ might not be asymptotic to any of the Mather sets involved. However, the behavior of $O(x)$ connects somehow these Mather sets which have different asymptotic behaviors.

Now, we apply Mather's theorem to geodesic flows on the two torus. Starting with a flat metric on $T^{2}$, we consider a $C^{k}$ perturbation of this metric for $k \geq 4$. There are regions of the geodesic flow of the perturbed metric that can be represented by a monotone twist map of an annulus $F: A \longrightarrow A$ as we observed before. Globally minimizing geodesics of the geodesic flow give rise to Mather sets of the twist map, and the asymptotic behavior of these geodesics determines the rotation number of the 
corresponding Mather set. However, we need to find a Birkhoff region of instability in this twist map to be able to apply Mather's result. The existence of a Birkhoff region of instability follows from the following lemma in $[90]$.

Lemma 7.3. Given $k>0$, the set of metrics in $T^{2}$ without invariant tori having rational rotation numbers is dense in the $C^{k}$ topology. In the $C^{2}$ topology, the above set is generic (i.e., an intersection of a countable collection of open and dense sets).

Claim: Given a $C^{\infty}$ metric $\bar{g}$ in $T^{2}$ such that

1. $\left(T^{2}, \bar{g}\right)$ is a $C^{4}$ perturbation of a flat metric in the torus,

2. The geodesic flow of $\left(T^{2}, \bar{g}\right)$ has no invariant tori of rational rotation number,

there exists an invariant region (in fact many of them) of the unit tangent bundle of $\left(T^{2}, \bar{g}\right)$ that can be represented by a monotone twist map of an annulus having a Birkhoff region of instability.

To show the claim it will be enough to find an open region of the unit tangent bundle bounded by two invariant tori of different rotation numbers, where there is no other invariant torus. Let us first recall that the collection of invariant tori varies continuously in the unit tangent bundle, as well as their corresponding rotation numbers (see [9] for instance for a nice exposition about invariant tori). We already know that there are lots of invariant tori if the perturbed metric is sufficiently close to the flat metric. But the absence of invariant tori with rational rotation numbers implies that invariant tori cannot be dense in the unit tangent bundle. Otherwise, since the collection of invariant tori is closed, every point in the unit tangent bundle would be contained in some invariant torus. In particular, a periodic orbit would be contained in an invariant torus, contradicting the absence of invariant tori with rational rotation numbers. Since the set of invariant tori of the perturbed metric is almost dense in the unit tangent bundle, there must be a pair of invariant tori close to each other and bounding a region where there is an open set $B$ not meeting any invariant curve. Let $A_{\rho_{1}, \rho_{2}}(B)$ be an invariant region of the unit tangent bundle bounded by two invariant tori whose rotation numbers are $\rho_{1}<\rho_{2}$, which contains $B$. The intersection of this collection of invariant regions gives rise to the desired Birkhoff region of instability.

Another way of proving that invariant tori cannot be dense in the unit tangent bundle of a perturbation of a flat metric in the torus is to use Birkhoff's theorem of invariant curves of twist maps. Indeed, identifying invariant tori with invariant curves, it is possible to show that invariant tori of perturbations of flat metrics are minimizing, in the sense that every 
geodesic contained in such a torus is globally minimizing [9]. Hence, if invariant tori are dense then every geodesic would be minimizing and thus the metric would have no conjugate points. But the only metrics in the torus without conjugate points are the flat metrics by Hopf's theorem. We leave the details to the reader.

The next step to show Theorem 7.3 consists in looking at the instability of geodesics of perturbations of a flat metric in the two torus produced by Mather's theorem.

Lemma 7.4. A flat metric in the two torus does not satisfy the $C^{k}$ shadowing property for any $k \geq 4$.

Proof. We just sketch the argument in [90]. Let us consider a $C^{4}$ perturbation $\left(T^{2}, \bar{g}\right)$ of a flat metric $\left(T^{2}, g\right)$ as described above. Mather's result implies that there exist a sequence (in fact many of them) $\omega_{i}, i \in \mathbb{Z}$ of different asymptotic directions; globally minimizing geodesics $\gamma_{i}$ in $\left(\mathbb{R}^{2}, \bar{g}\right)$ (the universal covering of $\left(T^{2}, \bar{g}\right)$ ) whose asymptotic directions are $\omega_{i}$; and a geodesic $\beta \in\left(\mathbb{R}^{2}, \bar{g}\right)$ such that large subsets $\beta\left[t_{i}, s_{i}\right]$ of $\beta$ are shadowed by the geodesics $\gamma_{i}$. If we assume that $\left(T^{2}, g\right)$ satisfies the $C^{k}$ shadowing property for $k \geq 4$, then the geodesic $\beta$ must have a shadow in the collection of geodesics of a flat metric, namely, straight lines in the plane. Since $\beta$ is within a finite distance from its shadow, $\beta$ has an asymptotic direction. On the other hand, $\beta$ follows, along large subsets, minimizing geodesics $\gamma_{i}$ with different asymptotic behaviors. By Hedlund's work [49] the geodesics $\gamma_{i}$ are shadowed by straight lines, and the distance of these geodesics to their shadows is uniform in $i \in \mathbb{Z}$. Therefore, we conclude that $\beta$ has a collection of large subsets $\beta\left[t_{i}, s_{i}\right]$ which are within finite distance of straight lines $L_{i}$ which diverge: they have different asymptotic behaviors. Using this fact we prove in [90] that $\beta$ cannot have a well defined asymptotic direction, contradicting the assumption.

The proof of Theorem 7.3 combines the previous lemma with the geometry of nonpositive curvature. Let $(M, g)$ be a compact manifold of nonpositive curvature containing an immersed, flat, totally geodesic torus $\left(T^{2}, g\right)$. Assume that the manifold satisfies the $C^{k}$ shadowing property for some $k \geq 4$. We use the same idea of Section 2 to get perturbations $(M, \bar{g})$ of $(M, g)$ which keep $\left(T^{2}, \bar{g}\right)$ totally geodesic, in a way that $\left(T^{2}, \bar{g}\right)$ satisfies Lemma 7.3. Then we show, by means of the convexity of nonpositive curvature geometry, that a geodesic of $\left(T^{2}, \bar{g}\right)$ that is shadowed by some geodesic in $(M, g)$ can be actually shadowed by a geodesic in $\left(T^{2}, g\right)$, thus reducing the problem to the two torus and Lemma 7.4 . 


\section{Chapter 8}

\section{Weak hyperbolicity of geodesics, global geometry and Poincaré conjecture}

The last chapter of the survey is devoted to discuss some generalizations of the theorems presented in Chapter 7 . Such generalizations are far from a complete answer to the conjecture settled in Chapter 7 , but show how to deal in some cases with the lack of convexity of the metric.

The first result concerning the conjecture for manifolds which might have regions of positive curvature involves the so-called manifolds with bounded asymptote defined in Chapter 4 (Definition 4.6). The theorem we shall discuss next is proved in [89].

Theorem 8.1. Let $(M, g)$ be a compact manifold without conjugate points and bounded asymptote with constant $B$. Let $\rho(M)$ be the injectivity radius of $(M, g)$. Assume that the geodesic flow is either topologically stable or possesses the $\epsilon-C^{k}$ shadowing property, where $\epsilon=\frac{1}{6 B} \rho(M)$. Then every abelian subgroup of the fundamental group of $M$ is infinite cyclic.

The bounded asymptote condition is weaker than the restriction of nonpositive curvature. However, it is difficult to check in practice and thus it is not a very satisfactory assumption. Yet, the $C^{0}$ techniques developed to deal with the absence of nonpositive curvature are very interesting and show how hard is to study dynamics and global geometry in the absence of convexity. Some results for a more general class of manifolds were proved for three dimensional manifolds in [92].

Theorem 8.2. Let $(M, g)$ be a compact three-dimensional manifold without conjugate points with the weak asymptoticity property, where $(\tilde{M}, g)$ is $K, C$ quasi-convex and $C<\frac{\rho(M)}{4}$. If $(M, g)$ satisfies the $\epsilon-C^{k}$ shadowing property 
for some $k>0$, and $\epsilon<\frac{1}{2 K}\left(\frac{\rho(M)}{2}-2 C\right)$, then $\pi_{1}(M)$ has the Preissmann property.

We say that a complete manifold without conjugate points $(M, g)$ satisfies the weak asymptoticity property if the map $\theta \rightarrow H_{\theta}(0)$ is continuous according to the definition given in Chapter 4 , Section 4 . Weak asymptoticity and quasi-convexity are properties of manifolds with bounded asymptote, according to Lemma 4.11. However, they are again difficult properties to verify in practice and they are usually related to uniformization theory (example: Corollary 4.1 in the case of surfaces).

Concerning the relationship between topological stability and the Preissmann property, we have to introduce the notion of strongly topologically stable.

Definition 8.1. We say that the geodesic flow of $(M, g)$ is strongly $C^{k}$ topologically stable for some $k>0$ if the geodesic flow of $(M, g)$ is $C^{k}$ topologically stable and the equivalences arising from the topological stability of the geodesic flow of $(M, g)$ act trivially in the homotopy group of $(M, g)$.

The above definition is based on the theory of hyperbolic systems: the theory of structural stability of Anosov systems implies that the conjugacies between such a system and neighboring systems are isotopic to the identity. Then, we can show the following:

Theorem 8.3. Let $(M, g)$ be a compact three-dimensional Riemannian manifold without conjugate points with the weak axiom of asymptoticity whose universal covering is $K, C$-quasi-convex, where $C<\frac{\rho(M)}{2}$. If the geodesic flow is strongly $C^{k}$ topologically stable then the fundamental group has the Preissmann's property.

Observe that in both Theorem 8.2 and Theorem 8.3 the quasi-convexity additive constant $C$ has an a priori bound. So Theorems 8.2 and 8.3 can be viewed as generalizations of the results in Chapter 7 for manifolds which are not far from being $K, 0$-quasi-convex.

The last section of the chapter will be devoted to discuss the connections of the theory presented in Chapters 6,7 and 8 with the recent work of Perelmann about the Poincaré conjecture and its relationship with Thurston's geometrization conjecture. We shall show that under certain usual topological assumptions, the Preissmann property implies the Gromov hyperbolicity of the universal covering of compact, connected three dimensional manifolds without conjugate points, provided that Thurston's geometrization is true. Moreover, Thurston's geometrization conjecture and the results of Chapter 6 would imply that compact, connected three dimensional manifolds without conjugate points and expansive geodesic flows admit metrics 
of negative curvature. So the results in Chapters 6,7 and 8 would give a relevant link between weak stability properties of the geodesic flow and uniformization theory in higher dimensions.

\section{Homoclinic geodesics in manifolds without conjugate points and variable curvature sign}

One of the main steps towards the proof of the conjecture for nonpositive curvature manifolds was the creation of homoclinic geodesics by perturbing the metric $(M, g)$. This problem is in itself interesting and hard, we shall devote this section to show in some detail some of the results on the subject. The main result of the section concerning this issue is the following [92] (see also [89]) which hold under quite general assumptions.

Proposition 8.1. Let $(M, g)$ be a compact Riemannian manifold without conjugate points such that $(\tilde{M}, \tilde{g})$ is quasi-convex. Assume that there exists a closed geodesic $\gamma$, and a lift $\bar{\gamma}$ of $\gamma$ in $\tilde{M}$, such that the centralizer of the covering isometry $T_{\bar{\gamma}}$ contains a subgroup isomorphic to $\mathbb{Z} \times \mathbb{Z}$. Then, given $\delta>0$ small and $k>0$, there exists a $\delta$ - $C^{k}$ conformal perturbation $\left(M, g_{\delta}\right)$ of $(M, g)$ satisfying the following properties:

1. The geodesic $\gamma$ is a $g_{\delta}$ geodesic that is a waist in its homotopy class with respect to the $g_{\delta}$-length.

2. Given a lift $\gamma_{1}$ of $\gamma$ in $\tilde{M}$, there exist a lift $\eta \neq \gamma_{1}$ of $\gamma$, and a $g_{\delta^{-}}$ geodesic $\beta$ with the following properties:

(a) $\lim _{t \rightarrow-\infty} d\left(\beta(t), \gamma_{1}\right)=0$ and $\lim _{t \rightarrow+\infty} d(\beta(t), \eta)=0$.

(b) If $\theta=\left(\gamma_{1}(0), \gamma_{1}^{\prime}(0)\right)$, then we have that

$$
\lim _{t \rightarrow+\infty} \angle\left(\beta^{\prime}(t),-\nabla b^{\theta}(\beta(t))\right)=0
$$

where $\angle(v, w)$ is the angle in the metric $g$ formed by the vectors $v, w$.

Proposition 8.1 is an interesting generalization of Morse-Hedlund two dimensional theory of existence of homoclinic geodesics. Observe that there is no restriction on the dimension of the manifold, there are no extra assumptions on the curvature and Jacobi fields, and the geodesic $\Pi(\beta)$ in $M$ is homoclinic to $\gamma$. However, the lemma does not grant that the lift $\gamma_{2}$ of $\gamma$ is an axis of the covering isometry $T_{\gamma_{1}}: \tilde{M} \longrightarrow \tilde{M}$ which translates $\gamma_{1}$, as in the case of surfaces.

We would like to make a remark that is connected with some of the questions posed at the end of the survey. The metric $g_{\delta}$ in Proposition 8.1 is 
a perturbation of the metric $g$ which has no conjugate points. Since $\pi_{1}(M)$ has an abelian subgroup of rank at least two, the geodesic flow cannot be Anosov because $\pi_{1}(M)$ for compact manifolds with Anosov geodesic flows is Gromov hyperbolic according to Chapter 6. Therefore, by virtue of Theorem 3.7 the metric $(M, g)$ is in the boundary of the set of metrics of $M$ without conjugate points and hence, the metric $g_{\delta}$ is likely to have conjugate points. The existence of conjugate points for $g_{\delta}$ is not part of the proof of Proposition 8.1, this feature of $g_{\delta}$ is not needed in that proof. However, the kind of homoclinic behavior of geodesics claimed in Proposition 8.1 is a strong sign of the existence of conjugate points in $\left(M, g_{\delta}\right)$, since in all known examples of manifolds without conjugate points such behavior is not possible. We think that homoclinic geodesics like in Proposition 8.1 should not exist in manifolds without conjugate points, but we do not know how to show this assertion. We shall come back to this issue in forthcoming sections.

Proposition 8.1 will play a key role in the study of the main conjecture in the case of manifolds whose curvature sign may change. Moreover, Proposition 8.1 is stated in [92] without proof. So we think that is interesting to show the main ideas of its proof with some detail due to its relevance for the rest of the survey.

\subsection{A generalization of Hedlund's tunnels}

The construction of the perturbations in Proposition 8.1 is motivated by some of the ideas that Hedlund [49] applied to investigate minimizing geodesics in certain metrics on the torus $T^{n}$. Hedlund changed the flat metric on $T^{n}$ in tubular neighbourhoods of closed geodesics generating the homotopy of $T^{n}, n \geq 3$, by shrinking drastically the metric on these neighbourhoods. This was to show the existence of homoclinic and heteroclinic, globally minimizing geodesics in the new metric. Such construction showed that there were examples of globally minimizing, homoclinic geodesics in $T^{n}, n \geq 3$, and showed new global behavior of minimizers in $T^{3}$ (heteroclinic geodesics). Now we known from Mather's theory that there exists heteroclinic minimizing geodesics in any non-flat metric in $T^{2}$ as well as we noticed in Chapter 7 .

Inspired by these Hedlund's tunnels, we construct in [89] perturbations of $(M, g)$ supported in a tubular neighborhood of a closed geodesic that force the globally minimizing geodesics of the perturbed metric to be most of the time in the support of the perturbation. Let us denote by $l_{g}\left(\gamma_{\theta}\right)$ the length of a closed geodesic $\gamma_{\theta}$ with respect to the metric $g$. The perturbation Lemma is the following:

Lemma 8.1. Let $(M, g)$ be a compact Riemannian manifold and let $[\gamma]$ be a nonzero free homotopy class represented by a minimizing closed geodesic 
$\gamma_{\theta}$, where $\theta \in T_{1} M$. Then, given $k \in N, \delta>0$, there exists a $\delta-C^{k}$ conformal perturbation $\left(M, g_{\delta}\right)$ of $(M, g)$ such that

1. $\gamma_{\theta}$ is a geodesic of $\left(M, g_{\delta}\right)$.

2. $l_{g_{\delta}}(\gamma)<l_{g}(\gamma)$.

3. Let $\tilde{g}_{\delta}$ be the pull-back of $g_{\delta}$ in $\tilde{M}$. Let $\gamma_{\tilde{\theta}}$ be a lift of $\gamma_{\theta}$ in $\tilde{M}$, where $\tilde{\theta} \in T_{1} \tilde{M}$. Let $\beta:[0,1] \longrightarrow \tilde{M}$ be a smooth curve such that $\beta(0) \in H_{\tilde{\theta}}(0), \beta(1) \in H_{\tilde{\theta}}\left(l_{g}\left(\gamma_{\theta}\right)\right)$. Then $l_{\tilde{g} \delta}\left(\gamma_{\tilde{\theta}}\right) \leq l_{\tilde{g} \delta}(\beta) \leq l_{\tilde{g}}(\beta)$.

4. Let $\beta$ be the curve defined in item (3). Then given $\epsilon>0$ there exists $a=a(\epsilon)$ such that if

$$
\left|l_{\tilde{g}_{\delta}}(\beta)-l_{\tilde{g}_{\delta}}\left(\gamma_{\tilde{\theta}}\right)\right| \leq a
$$

then $d_{H}\left(\beta, \gamma_{\tilde{\theta}}\left[0, l_{g}\left(\gamma_{\theta}\right)\right]\right) \leq \epsilon$, where $d_{H}$ is the Hausdorff distance in the metric $\tilde{g}$.

Proof. The proof follows the same line of reasoning in [89]. We give next a sketch of proof.

The main idea is to multiply the metric $g$ in by a factor function of the form $f_{\delta}(p)=\left(1-h_{\delta}(p)\right)^{2}$, where $h_{\delta}: M \longrightarrow \mathbb{R}$ is a $\delta$-small $C^{\infty}$ nonnegative function supported in a tubular neighborhood $V_{r}\left(\gamma_{\theta}\right)$ of $\gamma_{\theta}$ of small radius $r=r(\delta)$. We can choose $h_{\delta}$ with the following properties:

1. $h_{\delta}\left(\gamma_{\theta}(t)\right)=m_{\delta}>0$ for every $t \in\left[0, l_{g}\left(\gamma_{\theta}\right)\right]$, and $m_{\delta}$ is the maximum of $h_{\delta}$ in $M$.

2. $h_{\delta}(p)<m_{\delta}$ for every $p \notin \gamma_{\theta}$.

So the metric $\left(g_{\delta}\right)_{p}=f_{\delta}(p) g_{p}$ shrinks the length of the geodesic $\gamma_{\theta}$ :

$$
l_{g_{\delta}}\left(\gamma_{\theta}\right)=\left(1-m_{\delta}\right) l_{g}\left(\gamma_{\theta}\right) .
$$

To shorten notation, let us denote by $P$ the $g$-length of $\gamma_{\theta}$. Consider a lift $\gamma_{\tilde{\theta}}$ of $\gamma_{\theta}$ in the universal covering $\tilde{M}$. Take a smooth curve $\beta:[0,1] \longrightarrow \tilde{M}$ such that $\beta(0) \in H_{\tilde{\theta}}(0), \beta(1) \in H_{\tilde{\theta}}(P)$. Since the $\tilde{g}$-distance between the horospheres $H_{\tilde{\theta}}(0)$ and $H_{\tilde{\theta}}(P)$ is $P=l_{g}\left(\gamma_{\theta}\right)=l_{\tilde{g}}\left(\gamma_{\tilde{\theta}}[0, P]\right)$, we have that $l_{\tilde{g}}(\beta) \geq l_{\tilde{g}}\left(\gamma_{\tilde{\theta}}[0, P]\right)$. Moreover, we have equality in the previous inequality if and only if $\beta$ is a subset of an orbit of the Busemann flow of $\gamma_{\tilde{\theta}}$. Let us parametrize $\beta:[0, s] \longrightarrow \tilde{M}$ by $\tilde{g}$-arc length. We have

$$
\begin{aligned}
l_{\tilde{g}_{\delta}}(\beta) & =\int_{0}^{s}\left(1-h_{\delta}(\beta(t))\right) \sqrt{\tilde{g}\left(\beta^{\prime}(t), \beta^{\prime}(t)\right)} d t \\
& =l_{\tilde{g}}(\beta)-\int_{0}^{s} h_{\delta}(\beta(t)) d t .
\end{aligned}
$$


So we get

$$
\begin{aligned}
l_{\tilde{g}_{\delta}}(\beta)-l_{\tilde{g}_{\delta}}\left(\gamma_{\tilde{\theta}}[0, P]\right)= & l_{\tilde{g}}(\beta)-l_{\tilde{g}}\left(\gamma_{\tilde{\theta}}[0, P]\right) \\
& -\left(\int_{0}^{s} h_{\delta}(\beta(t)) d t-\int_{0}^{P} h_{\delta}\left(\gamma_{\tilde{\theta}}(t)\right) d t\right) .
\end{aligned}
$$

Namely,

$$
\begin{aligned}
l_{\tilde{g}_{\delta}}(\beta)-l_{\tilde{g}_{\delta}}\left(\gamma_{\tilde{\theta}}[0, P]\right) & =s-P-\left(\int_{0}^{s} h_{\delta}(\beta(t)) d t-P m_{\delta}\right) \\
& =s-P-\left(\int_{0}^{s}\left(h_{\delta}(\beta(t))-m_{\delta}\right) d t+(s-P) m_{\delta}\right) \\
& =(s-P)\left(1-m_{\delta}\right)+\int_{0}^{s}\left(m_{\delta}-h_{\delta}(\beta(t)) d t .\right.
\end{aligned}
$$

The terms $(s-P)\left(1-m_{\delta}\right)$ and $\int_{0}^{s}\left(m_{\delta}-h(\beta(t)) d t\right.$ are both non negative, since $m_{\delta}$ is the maximum value of $h_{\delta}$ and $s \geq P$. It is clear that their sum goes to zero if and only if $s=l_{\tilde{g}}(\beta)$ tends to $P$ and $h_{\delta}(\beta(t))$ tends to $m_{\delta}$. By the choice of $h_{\delta}$, this can only happen if $\beta[0, s]$ tends to $\gamma_{\tilde{\theta}}[0, P]$ in the Hausdorff distance.

The fact that $\gamma_{\theta}$ is still a $g_{\delta}$-geodesic can be checked in many ways. This is a consequence, for instance, of the conformal connection formula and the fact that the function $f$ is critical along $\gamma_{\theta}$ (see [88], [89] for details). Another way to show this fact is to use item (3) of the lemma. Indeed, item (3) implies that $\gamma_{\theta}$ is a $g_{\delta}$-minimizer in its homotopy class. Because if a closed curve $c \subset M$ is homotopic to $\gamma_{\theta}$, it has a lift $\tilde{c}$ within a finite Hausdorff distance from the lift $\gamma_{\tilde{\theta}}$ of $\gamma_{\theta}$. This yields that $\tilde{c}$ meets every horosphere $H_{\tilde{\theta}}(t), t \in \mathbb{R}$, so we can apply item (3) to compare the $g_{\delta}$-length of periodic pieces of $\tilde{c}$ with the length of $\gamma_{\theta}$. We leave the details to the reader.

\subsection{On finding homoclinic, minimizing geodesics in perturbed metrics}

Now, let $[\gamma]$ be a nontrivial free homotopy class represented by a closed geodesic $\gamma_{\theta}$. Let us assume that there is a lift $\gamma_{\tilde{\theta}}$ of $\gamma=\gamma_{\theta}$ such that the covering isometry $T=T_{\gamma_{\tilde{\theta}}}$ has nontrivial centralizer $Z([\gamma])$, i.e., the quotient $Z([\gamma]) /\langle T\rangle$ is not the identity. The notation $d_{g}(p, A)$ means the $g$-distance from a point $p$ to a set $A$. If $\gamma, \beta$ are two geodesics in $\tilde{M}$, the notation $d_{H}(\gamma, \beta)$ means the Hausdorff distance between $\gamma$ and $\beta$ in the metric $\tilde{g}$. Notice that since any two metrics in $M$ are equivalent, the Hausdorff distances associated to two metrics $\tilde{g}, \tilde{h}$ obtained as pull backs of metrics in $M$ are equivalent too. So in general, we shall not refer to the 
metric $g$ in the notation $d_{H}$ of the Haussdorff distance unless it is strictly necessary. Sometimes we shall use the notation $d($,$) , meaning d_{g}$. We shall always use the notation $d_{h}$ whenever $h$ is a metric different from $g$.

We apply Lemma 8.1 to $\gamma_{\theta}$ to get a metric $g_{\delta}$ in $M$ satisfying all the properties of the lemma. In particular, $\gamma_{\theta}$ is a $g_{\delta}$-geodesic which is a waist in its homotopy class.

Recall that the length $l_{g}\left(\gamma_{\theta}\right)$ is the positive, minimum period of $\gamma_{\theta}$ in the metric $g$. We shall always assume that the displacement of $T$ along $\gamma_{\tilde{\theta}}$ is $l_{g}\left(\gamma_{\theta}\right)$ (in general, the displacement of covering isometries preserving $\gamma_{\tilde{\theta}}$ is an integer multiple of $\left.l_{g}\left(\gamma_{\theta}\right)\right)$.

Let $b \in Z([\gamma]) \backslash\langle T\rangle$ and define $\gamma_{\tilde{\eta}}=T_{b}\left(\gamma_{\tilde{\theta}}\right)$. The geodesic $\gamma_{\tilde{\eta}}$ is biasymptotic to $\gamma_{\tilde{\theta}}$ according to Lemma 6.1 , i.e., there exists $L=L(b)>0$ such that $d\left(\gamma_{\tilde{\theta}}, \gamma_{\tilde{\eta}}\right) \leq L$, because $T$ and $T_{b}$ commute. Parametrize both geodesics in a way that $\gamma_{\tilde{\eta}}(t)=\gamma_{\tilde{\eta}} \cap H_{\tilde{\theta}}(t)$.

Call $\beta_{n}$ the geodesic of $\left(\tilde{M}, g_{\delta}\right)$ that attains the distance between $\gamma_{\tilde{\theta}}\left(-n l_{g}\left(\gamma_{\theta}\right)\right)$ and $\gamma_{\tilde{\eta}}\left(n l_{g}\left(\gamma_{\theta}\right)\right)$. We shall show that a subsequence of the $\beta_{n}$ approaches the lift $\beta_{0}$ of a homoclinic geodesic $\beta$ of $\gamma_{\theta}$. This procedure generalizes the standard method used in surfaces by Morse to show the existence of homoclinic geodesics.

We shall always parametrize $\beta_{n}:\left[0, L_{n}\right] \longrightarrow \tilde{M}$ by $\tilde{g}_{\delta}$-arclength, where $\beta_{n}(0)=\gamma_{\tilde{\theta}}\left(-n l_{g}\left(\gamma_{\theta}\right)\right)$ and $\beta_{n}\left(L_{n}\right)=\gamma_{\tilde{\eta}}\left(n l_{g}\left(\gamma_{\theta}\right)\right)$. We shall denote by $d_{T_{1} \tilde{M}}(v, w)$ the Sasaki distance associated to $\tilde{g}$ in the unit tangent bundle of $\tilde{M}$. The following notion was introduced in [87].

Definition 8.2. Given $\epsilon>0, \theta \in T_{1} M$, and a lift $\gamma_{\tilde{\theta}}$ of $\gamma_{\theta}$ in $\tilde{M}$, we say that a $\tilde{g}_{\delta}$-geodesic segment $\alpha:(a, b) \longrightarrow \tilde{M}$ is $\epsilon, \tilde{\theta}$-critical if the following two conditions hold:

1. $\angle\left(\alpha^{\prime}(t),-\nabla b^{\tilde{\theta}}(\alpha(t))\right) \leq \epsilon$ for every $t \in(a, b)$, where the angle $\angle($,$) is$ measured with respect to the metric $g$.

2. There exists a lift $\gamma_{\tilde{\eta}}$ of $\gamma_{\theta}$ which can be parametrized so that $d_{T_{1} \tilde{M}}\left(\alpha^{\prime}(t), \gamma_{\tilde{\eta}}^{\prime}(t)\right) \leq \epsilon$ for every $t \in(a, b)$.

A $0, \tilde{\theta}$-critical geodesic segment is a subset of a lift of $\gamma_{\theta}$ that is an orbit of the Buseman flow $\psi^{\tilde{\theta}}$ (recall that every lift of $\gamma_{\theta}$ is also a $g_{\delta}$-geodesic by the construction of $g_{\delta}$ ). In particular, this lift is asymptotic to $\gamma_{\tilde{\theta}}$ and therefore, by Lemma 1.3 in [89], it is an axis of $T$.

Lemma 8.2. A $g_{\delta}$-geodesic segment $\eta:(a, b) \longrightarrow \tilde{M}$ that is a limit of $\epsilon, \tilde{\theta}$ critical segments is an $\epsilon, \tilde{\theta}$-critical geodesic segment. Moreover, if $\alpha(a, b)$ is $\epsilon, \tilde{\theta}$-critical then $(T)^{k}(\alpha(a, b))$ is also $\epsilon, \tilde{\theta}$-critical for every $k \in \mathbb{Z}$. 
Proof. Since the proof is easy, we just give an outline for the sake of completeness. The first assertion of the Lemma follows from the fact that items (1) and (2) in Definition 8.2 are given by closed conditions. The second assertion follows from two facts. First of all, the map $T$ is an isometry of $(\tilde{M}, \tilde{g})$ that preserves the Busemann flow $\psi^{\tilde{\theta}}$, i.e., $(D T)^{k}\left(-\nabla_{p} b^{\tilde{\theta}}\right)=$ $-\nabla_{T(p)} b^{\tilde{\theta}}$, so $T$ preserves the $g$-angles of curves with respect to $-\nabla b_{\tilde{\theta}}$.

Secondly, the representation of $\pi_{1}(M)$ in the group of diffeomorphisms of $\tilde{M}$ acts by isometries in both $(\tilde{M}, \tilde{g})$ and $\left(\tilde{M}, \tilde{g}_{\delta}\right)$. This is just by the definition of such representation: the action of $\pi_{M}$ in $\tilde{M}$ is topological and intrinsic, it is obtained by lifting closed curves in $M$. Clearly, this is independent of the metric in $M$, so $T$ is an isometry of $\left(\tilde{M}, \tilde{g}_{\delta}\right)$.

Now, if $\alpha(a, b)$ is a $\tilde{g}_{\delta}$-geodesic segment, then $T^{k}(\alpha(a, b))$ is a $\tilde{g}_{\delta}$-geodesic segment for every $k \in \mathbb{Z}$. Moreover, if $\alpha^{\prime}(t)$ is close to the vector field $-\nabla b_{\tilde{\theta}}$ for every $t \in(a, b)$, then $D T^{k}\left(\alpha^{\prime}(t)\right)$ remains close to the vector field $D T^{k}\left(-\nabla b^{\tilde{\theta}}\right)=-\nabla b^{\tilde{\theta}}$ for every $t \in(a, b)$. Furthermore, if $\alpha(a, b)$ is close to a lift $\beta$ of $\gamma_{\theta}$, then $(T)^{k}(\alpha(a, b))$ is close to $T(\beta)$ that is a lift of $\gamma_{\theta}$. This concludes the proof of the Lemma.

Lemma 8.3. There exists $\epsilon_{0}$ such that, if $\epsilon \leq \epsilon_{0}$ and two consecutive $\tilde{g}_{\delta}$-geodesic segments $\alpha(a, b), \alpha(b, c)$ are $\epsilon, \tilde{\theta}$-critical, then $\alpha(a, c)$ is also $\epsilon, \tilde{\theta}$-critical.

Proof. If $\alpha(a, b), \alpha(b, c)$ are $\epsilon, \tilde{\theta}$-critical, then $\angle\left(\alpha^{\prime}(t),-\nabla_{\alpha(t)} b^{\tilde{\theta}}\right) \leq \epsilon$ for every $t \in(a, b) \cup(b, c)$, and there exist two lifts $\beta, \sigma$ of $\gamma$ such that

$$
d_{T_{1} \tilde{M}}\left(\alpha^{\prime}(t), \beta^{\prime}(t)\right) \leq \epsilon
$$

for every $t \in(a, b)$,

$$
d_{T_{1} \tilde{M}}\left(\alpha^{\prime}(t), \sigma^{\prime}(t)\right) \leq \epsilon
$$

for every $t \in(b, c)$. The point is that if $\epsilon$ is small enough, then $\beta=\sigma$. Indeed, since the action of $\pi_{1}(M)$ is discrete in $\tilde{M}$, there exists $\epsilon_{1}>0$ such that if two lifts $\sigma_{1}, \sigma_{2}$ of $\gamma_{\theta}$ have points at distance less than $\epsilon_{1}$, then either $\sigma_{1}=\sigma_{2}$, or $\sigma_{1}$ intersects $\sigma_{2}$ transversally at some point making an angle of at least $\epsilon_{1}$. This clearly implies that there exists $\epsilon_{2}\left(\epsilon_{1}\right)>0$ such that, given two lifts $\sigma_{1}, \sigma_{2}$ of $\gamma$, then either $\sigma_{1}=\sigma_{2}$, or $d_{T_{1} \tilde{M}}\left(\sigma_{1}^{\prime}(t), \sigma_{2}^{\prime}(t)\right)>\epsilon_{2}$ for every $t \in \mathbb{R}$. Now, let $\epsilon_{0}=\frac{\epsilon_{2}}{2}$. If $d_{T_{1} \tilde{M}}\left(\alpha^{\prime}(t), \beta^{\prime}(t)\right) \leq \epsilon_{0}$ for every $t \in(a, b)$, and $d_{T_{1} \tilde{M}}\left(\alpha^{\prime}(t), \sigma^{\prime}(t)\right) \leq \epsilon_{0}$ for every $t \in(b, c)$, then

$$
d_{T_{1} \tilde{M}}\left(\beta^{\prime}(b), \sigma^{\prime}(b)\right) \leq 2 \epsilon_{0} \leq \epsilon_{2},
$$

and hence $\beta=\sigma$ according to the definition of $\epsilon_{2}$. This implies that the geodesic segment $\alpha(a, c)$ is within a distance $\epsilon_{0}$ from a single lift of $\gamma_{\theta}$, thus concluding the proof of the Lemma. 
From now on, we assume that the number $r=r(\delta)$, that is the radius of the support $V_{r}\left(\gamma_{\theta}\right)$ of $g_{\delta}$, is smaller than $\epsilon_{0}$. To simplify the notation during this section, let us call by $H_{k}$ the horosphere of $\gamma_{\tilde{\theta}}$ in $(\tilde{M}, \tilde{g})$ containing the point $\gamma_{\tilde{\theta}}\left(k l_{g}\left(\gamma_{\theta}\right)\right)$. Take a partition of $\beta_{n}$ by connected segments $\beta_{n}\left[t_{i}, t_{i+1}\right]$, for $-n \leq i \leq n$, such that $\beta_{n}\left(t_{i}\right) \in H_{i}$ and $t_{i}<t_{i+1}$.

Lemma 8.4. For each $0<\epsilon \leq \epsilon_{0}$ there is a number $N(\epsilon)$ such that, for any $n \in \mathbb{N}$, all except at most $N(\epsilon)$ of the segments $\beta_{n}\left(\left[t_{i}, t_{i+1}\right]\right)$ are $\epsilon, \tilde{\theta}$-critical. Furthermore, the complement of the union of the $\epsilon, \tilde{\theta}$-critical segments $\beta_{n}\left(\left[t_{i}, t_{i+1}\right]\right)$ has $\tilde{g}_{\delta}$-length bounded above by $N(\epsilon)\left(l_{g_{\delta}}\left(\gamma_{\theta}\right)+L\right)$, where $L$ was defined by $d_{H}\left(\gamma_{\tilde{\theta}}, T_{b}\left(\gamma_{\tilde{\theta}}\right)\right) \leq L$.

Proof. By horosphere geometry we have that the $\tilde{g}$-distance between $H_{i}$ and $H_{i+m}$ is equal to $m l_{g}\left(\gamma_{\theta}\right)=m l$, so

$$
l_{\tilde{g}}\left(\beta_{n}\left[t_{i}, t_{i+1}\right]\right) \geq l_{g}\left(\gamma_{\theta}\right)
$$

for every $i \in[-n, n]$. This means by Lemma 8.1 (3) that

$$
l_{g_{\delta}}\left(\beta_{n}\left[t_{i}, t_{i+1}\right]\right) \geq l_{g_{\delta}}(\gamma)
$$

for every $i$. From the fact that $\beta_{n}$ is $g_{\delta}$-globally minimizing and the triangle inequality we obtain

$$
l_{g_{\delta}}\left(\beta_{n}\right)=\sum_{i=-n}^{n-1} l_{g_{\delta}}\left(\beta_{n}\left[t_{i}, t_{i+1}\right]\right) \leq 2 n l_{g_{\delta}}\left(\gamma_{\theta}\right)+2 L,
$$

which implies that

$$
0 \leq \sum_{i=-n}^{n-1}\left[l_{g_{\delta}}\left(\beta_{n}\left[t_{i}, t_{i+1}\right]\right)-l_{g_{\delta}}\left(\gamma_{\theta}\right)\right] \leq 2 L .
$$

The above inequality has strong consequences. Since each term $\left[l_{g_{\delta}}\left(\beta_{n}\left[t_{i}, t_{i+1}\right]\right)-l_{g_{\delta}}(\gamma)\right]$ is positive, the fact that their sum is bounded by $2 L$ for all $n$ implies that given $\lambda>0$ there exists $N=N(\lambda, \delta, L)$ and a subset $A_{N, n}$ of indexes, with $\operatorname{card}\left(A_{N, n}\right) \leq N$, such that

$$
\left|l_{g_{\delta}}\left(\beta_{n}\left[t_{i}, t_{i+1}\right]\right)-l_{g_{\delta}}(\gamma)\right| \leq \lambda
$$

for every $i \in[-n, n] \backslash A_{N, n}$.

Claim 1: Given $\lambda>0$ there exists $\mu=\mu(\lambda)>0$, and covering isometries $a_{i, n} \in \pi_{1}(M)$, such that, if $\left|l_{g_{\delta}}\left(\beta_{n}\left[t_{i}, t_{i+1}\right]\right)-l_{g_{\delta}}\left(\gamma_{\theta}\right)\right| \leq \lambda$, then

$$
d_{T_{1} \tilde{M}}\left(\beta_{n}^{\prime}(t), a_{i, n}\left(\gamma_{\tilde{\theta}}^{\prime}(x(t))\right)\right) \leq \mu,
$$

and

$$
\angle\left(\beta_{n}^{\prime}(t),-\nabla b^{\tilde{\theta}}\left(\beta_{n}(t)\right)\right) \leq \mu,
$$


for every $t \in\left[t_{i}, t_{i+1}\right]$, where $x(t) \in \mathbb{R}$.

Otherwise, the contradiction of the hypothesis of Claim 1 implies that there exists $t \in\left[t_{i}, t_{i+1}\right]$ such that, either $d\left(\beta_{n}(t), a\left(\gamma_{0}\right)\right)>\mu$ for every $a \in \pi_{1}(M)$, or the angle between $\beta_{n}^{\prime}(t)$ and $-\nabla b^{\theta}\left(\beta_{n}(t)\right)$ is at least $\mu$. In the first case, there exists $\alpha(\mu, \delta)>0$ such that

$$
\left[l_{g_{\delta}}\left(\beta_{n}\left[t_{i}, t_{i+1}\right]\right)-l_{g_{\delta}}\left(\gamma_{\theta}\right)\right]>\alpha(\mu, \delta),
$$

by Lemma $8.1,(3)$. In the latter case, there exists $\omega(\mu)>0$ such that $\left[l_{g}\left(\beta_{n}\left[t_{i}, t_{i+1}\right]\right)-l_{g}\left(\gamma_{\theta}\right)\right]>\omega(\mu)$, since the $g$-distance between $H_{i}$ and $H_{i+1}$ is attained at the asymptotes of $\gamma_{\tilde{\theta}}$ and equals $l=l_{g}\left(\gamma_{\theta}\right)$. Thus, again by Lemma 8.1, there exists $\bar{\alpha}(\mu, \delta)>0$ such that

$$
\left[l_{g_{\delta}}\left(\beta_{n}\left[t_{i}, t_{i+1}\right]\right)-l_{g_{\delta}}\left(\gamma_{\theta}\right)\right]>\bar{\alpha} .
$$

But, the number of indexes $i \in[-n, n-1]$ such that $\left[t_{i}, t_{i+1}\right]$ satisfies either (2) or (3) is bounded above by $N(\inf \{\alpha, \bar{\alpha}\})$. It is clear that $\lim _{\lambda \rightarrow 0} \mu(\lambda)=0$. This finishes the proof of Claim 1 .

As a consequence of the Claim we get that, given $\epsilon>0$ there exists $\lambda=\lambda(\epsilon)$ such that $\mu(\lambda) \leq \epsilon$, since $\lim _{\lambda \rightarrow 0} \mu(\lambda)=0$. Let

$$
\left.I_{n}^{1}=I_{n}^{1}(\epsilon)=\left\{\beta_{n}(t) \text { s.t. } d_{T_{1} \tilde{M}}\left(\beta_{n}^{\prime}(t), \sigma^{\prime}\right)\right)>\epsilon \forall \text { lift } \sigma \text { of } \gamma_{\theta}\right\} .
$$

and

$$
I_{n}^{2}=I_{n}^{2}(\epsilon)=\left\{\beta_{n}(t) \text { s.t. } \angle\left(\beta_{n}^{\prime}(t),-\nabla b^{\theta}\left(\beta_{n}(t)\right)\right)>\epsilon\right\} .
$$

In the definition of $I^{1}$, the notation $\sigma^{\prime}$ where $\sigma$ is a lift of $\gamma_{\theta}$ stands for the orbit of $\sigma$ under the geodesic flow. Let $I_{n}=I_{n}^{1} \cup I_{n}^{2}$. By inequality (1), and Claim 1, the cardinality of the collection of the $\beta_{n}\left[t_{i}, t_{i+1}\right]$ that contain points of $I_{n}$ is bounded above by $A_{N, n} \leq N=N(\lambda(\epsilon))=N(\epsilon)$. Moreover, by inequality $(7.1), l_{g_{\delta}}\left(\beta_{n}\left[t_{i}, t_{i+1}\right]\right) \leq l_{g_{\delta}}\left(\gamma_{\theta}\right)+2 L$, and hence, the length of the union of the $\beta_{n}\left[t_{i}, t_{i+1}\right]$ having points in $I_{n}$ is bounded by $N\left(l_{g_{\delta}}(\gamma)+2 L\right)$ (that is a number independent of $n$ ). Finally, observe that if $\beta_{n}\left[t_{i}, t_{i+1}\right]$ does not contain points of $I_{n}$ then $\beta_{n}\left[t_{i}, t_{i+1}\right]$ is an $\epsilon, \theta$-critical geodesic segment. This finishes the proof of the Lemma.

Lemma 8.4 motivates the following definition (taken from [89]).

Definition 8.3. Given $\epsilon>0$, we say that a $\tilde{g}_{\delta}$-geodesic segment $\alpha:(a, b) \longrightarrow \tilde{M}$ is mostly $\epsilon, \tilde{\theta}$-critical if there exist an integer $r \leq N(\epsilon)$, and a collection of intervals $\left[c_{1}, d_{1}\right], . .,\left[c_{r}, d_{r}\right]$ such that

1. $a \leq c_{1}<d_{1}<c_{2}<\ldots<c_{r}<d_{r} \leq b$.

2. Each curve $\alpha\left(\left[c_{i}, d_{i}\right]\right)$ is $\epsilon, \tilde{\theta}$-critical. 
3. the $\tilde{g}_{\delta}$-length of the complement of the set $\cup_{i=1}^{r} \alpha\left(\left[c_{i}, d_{i}\right]\right)$ is at most $N(\epsilon)\left(l_{g_{\delta}}(\gamma)+2 L\right)=Q(\epsilon)$.

Clearly, an $\epsilon, \tilde{\theta}$-critical geodesic segment is mostly $\epsilon, \tilde{\theta}$-critical.

Corollary 8.1. Given $0<\epsilon \leq \epsilon_{0}$, the geodesic segment $\beta_{n}$ is mostly $\epsilon, \tilde{\theta}$-critical for every $n \in \mathbb{N}$.

Proof. According to Lemma 8.4, each $\beta_{n}$ contains at most $r_{n} \leq N(\epsilon)$ intervals $\beta_{n}\left[t_{i_{j}}, t_{i_{j}+1}\right], 0 \leq j \leq r_{n}$, which are not $\epsilon, \tilde{\theta}$-critical. The total $\tilde{g}_{\delta}$-length of these geodesic segments is at most $Q(\epsilon)$. Each connected component in the complement of this collection is a union of $\epsilon, \tilde{\theta}$-critical $\tilde{g}_{\delta}$-geodesic segments. Since $\epsilon \leq \epsilon_{0}$ by hypothesis, Lemma 7.3 implies that such a component is $\epsilon, \theta$-critical, thus proving the corollary.

Corollary 8.1 improves Lemma 8.4: the geodesic segments $\beta_{n}$ are most of the time (up to a subset of length $\leq Q(\epsilon)$ ) close to the vector field $-\nabla b^{\tilde{\theta}}$, and simultaneously close to a certain number of lifts of $\gamma$. This number of lifts is bounded above by $N(\epsilon)$, a number which does not depend on $n$.

Proposition 8.2. There exists a subsequence of the $\beta_{n_{k}}$ satisfying the following properties:

1. There exists covering isometries $F_{k}$ such that the sequence of $\tilde{g}_{\delta}$ geodesics $\bar{\beta}_{k}=F_{k}\left(\beta_{n_{k}}\right)$ converges to a globally minimizing $\tilde{g}_{\delta}$-geodesic

$$
\beta:(-\infty, \infty) \longrightarrow \tilde{M}
$$

2. There exists a lift $\eta$ of $\gamma_{\theta}$ in $\tilde{M}$, that is different from $\gamma_{\tilde{\theta}}$ such that

$$
\begin{aligned}
& \lim _{t \rightarrow-\infty} d\left(\gamma_{\tilde{\theta}}, \beta(t)\right)=0, \\
& \lim _{t \rightarrow+\infty} d(\eta, \beta(t))=0 .
\end{aligned}
$$

Proof. The proof of the lemma is based on the ideas in [89], where the hypothesis of bounded asymptote is assumed (and hence, the conclusion of the lemma is stronger: we get that $\eta$ is in fact bi-asymptotic to $\gamma_{\tilde{\theta}}$ ). We shall show that we do not need the bounded asymptote hypothesis to prove the lemma. The first remark is that

Claim 1: Any limit of mostly $\epsilon, \tilde{\theta}$-critical geodesic segments is mostly $\epsilon, \tilde{\theta}$-critical. Also, the image of a mostly $\epsilon, \theta$-critical geodesic segment by any power of $T$ is a mostly $\epsilon, \tilde{\theta}$-critical geodesic segment.

The second important remark is the following result proved in [89] (Lemma 4.4): 
Lemma 8.5. Let $\gamma_{\tilde{\theta}}, T=T_{\gamma_{\tilde{\theta}}} \in \pi_{1}(M), T_{b}\left(\gamma_{\tilde{\theta}}\right)$, and $\beta_{n}$ be as before. Suppose that given $\epsilon>0$ there exists $n_{0}>0$ such that for every $n \geq n_{0}$ we have an interval $\left[s_{n}, r_{n}\right]$-with $\left|s_{n}-r_{n}\right| \geq n$ - and an axis $\Gamma$ of $T$ such that $d_{H}\left(\beta_{n}\left(\left[s_{n}, r_{n}\right]\right), \Gamma\right) \leq \epsilon$. Then the angle formed by $\beta_{n}$ and $\gamma_{\tilde{\theta}}$ at $\beta_{n}(0)$ must tend to zero as $n \rightarrow+\infty$.

The proof of Lemma 8.5 is based on a well known shortcut argument used by Morse [69] to study globally minimizing geodesics in surfaces. We show in [89] that this argument can be applied in this $n$-dimensional case. From this lemma we get:

Claim 2: Let $\beta_{n}:\left[0, L_{n}\right] \longrightarrow \tilde{M}$, where $\beta_{n}(0) \in \gamma_{\tilde{\theta}}$, and $\beta_{n}\left(L_{n}\right) \in T_{b}\left(\gamma_{\tilde{\theta}}\right)$. Let $\beta\left(\left[c_{i}^{n}, d_{i}^{n}\right]\right)$ be the $\epsilon, \tilde{\theta}$-critical subsets of $\beta_{n}$ given by Corollary 8.1. If there exists a subsequence $n_{k} \rightarrow+\infty$ such that

$$
d_{H}\left(\beta\left(\left[c_{i}^{n_{k}}, d_{i}^{n_{k}}\right]\right), \gamma_{\tilde{\theta}}\right) \rightarrow 0,
$$

then the angle between the $T$-axis $T_{b}\left(\gamma_{\tilde{\theta}}\right)$ and $\beta_{n_{k}}$ at $\beta_{n_{k}}\left(L_{n_{k}}\right)$ must tend to 0 as $k \rightarrow+\infty$. Analogously, if the sequence of curves $\beta\left(\left[c_{i}^{n_{k}}, d_{i}^{n_{k}}\right]\right)$ approaches $T_{b}\left(\gamma_{\tilde{\theta}}\right)$ then the angle between $\gamma_{\tilde{\theta}}$ and $\beta_{n_{k}}$ at $\beta_{n_{k}}(0)$ tends to 0 as $k$ tends to $+\infty$.

Claim 3: Assume that $(\tilde{M}, \tilde{g})$ is $K, C$-quasi-convex. Then the sequence $\beta_{n} \epsilon$-approaches (in the sense of Definition 8.3) at least two different lifts of $\gamma_{\theta}$.

Notice that the quasi-convexity assumption in Proposition 8.1 has not been used so far, this is the first place in the proof of the proposition where it shall be required.

Indeed, by Corollary 8.1 the geodesics $\beta_{n}$ are most of the time close to some lift of $\gamma_{\theta}$. By Lemma 8.5 we know that if the sequence $\beta_{n}$ approaches either $\gamma_{\tilde{\theta}}$ or $T_{b}\left(\gamma_{\tilde{\theta}}\right)$ then the sequence $\beta_{n}$ must approach both of them (and Claim 3 holds).

So let us suppose that the $\epsilon, \theta$-critical subsets of the sequence $\beta_{n}$ get close to neither $\gamma_{\tilde{\theta}}$ nor $T_{b}\left(\gamma_{\tilde{\theta}}\right)$.

Under this condition, assume by contradiction that that there exists a subsequence $\beta_{n_{i}}$ which comes close to just one lift $\gamma_{i}$ of $\gamma_{\theta}$. More precisely, assume that there exist $\epsilon>0, B=B(\epsilon)>0$, a subsequence $\beta_{n_{i}}$, a sequence of lifts $\gamma_{i}$ of $\gamma_{\theta}$ which are different from $\gamma_{\tilde{\theta}}$ and $T_{b}\left(\gamma_{\tilde{\theta}}\right)$, such that

1. The $\epsilon, \theta$-critical subsets $\beta_{n_{i}}\left(\left[c_{j}^{n_{i}}, d_{j}^{n_{i}}\right]\right)$ of $\beta_{n_{i}}\left(\left[0, L_{n_{i}}\right]\right)$, where $1 \leq j \leq$ $N_{i}$, satisfy

$$
d_{H}\left(\beta_{n_{i}}\left(\left[c_{j}^{n_{i}}, d_{j}^{n_{i}}\right]\right), \gamma_{i}\right) \leq \epsilon,
$$

for every $0 \leq j \leq N_{i} \leq N(\epsilon)$, where $N(\epsilon)$ is the number defined in Definition 8.3. 
2. The length of the complement in $\beta_{n_{i}}\left(\left[0, L_{n_{i}}\right]\right)$ of the union of the $\epsilon, \theta$-critical subsets is bounded above by $B$.

Notice that, since $L_{n_{i}}$ goes to $+\infty$ with $i$, assumptions (1) and (2) imply that

$$
\lim _{i \rightarrow+\infty}\left|c_{1}^{n_{i}}-d_{N_{i}}^{n_{i}}\right|=+\infty
$$

Let $a_{i}, b_{i}$ be such that

1. $d\left(\gamma_{i}\left(a_{i}\right), \beta_{n_{i}}\left(c_{1}^{n_{i}}\right)\right) \leq \epsilon$,

2. $d\left(\gamma_{i}\left(b_{i}\right), \beta_{n_{i}}\left(d_{N_{i}}^{n_{i}}\right)\right) \leq \epsilon$,

The contradiction assumptions and the triangle inequality imply that

$$
\begin{aligned}
d\left(\gamma_{i}\left(a_{i}\right), \gamma_{\tilde{\theta}}\right) & \leq d\left(\gamma_{i}\left(a_{i}\right), \beta_{n_{i}}\left(c_{1}^{n_{i}}\right)\right)+d\left(\beta_{n_{i}}\left(c_{1}^{n_{i}}\right), \gamma_{\tilde{\theta}}\right) \\
& \leq \epsilon+d\left(\beta_{n_{i}}\left(c_{1}^{n_{i}}\right), \beta_{n_{i}}(0)\right) \\
& \leq \epsilon+B .
\end{aligned}
$$

Analogously,

$$
\begin{aligned}
d\left(\gamma_{i}\left(b_{i}\right), T_{b}\left(\gamma_{\tilde{\theta}}\right)\right) & \leq d\left(\gamma_{i}\left(b_{i}\right), \beta_{n_{i}}\left(c_{N_{i}}^{n_{i}}\right)\right)+d\left(\beta_{n_{i}}\left(c_{N_{i}}^{n_{i}}\right), T_{b}\left(\gamma_{\tilde{\theta}}\right)\right) \\
& \leq \epsilon+B
\end{aligned}
$$

This yields

$$
\max \left\{d_{\tilde{g}}\left(\gamma_{i}\left(a_{i}\right), \gamma_{\tilde{\theta}}\right), d_{\tilde{g}}\left(\gamma_{i}\left(b_{i}\right), \gamma_{\tilde{\theta}}\right)\right\} \leq B+\epsilon+P,
$$

where $P$ is an upper bound for the distance between $\gamma_{\tilde{\theta}}$ and $T_{b}\left(\gamma_{\tilde{\theta}}\right)$.

By the $K, C$-quasi convexity of $(\tilde{M}, \tilde{g})$, we get that

$$
d_{H}\left(\gamma_{i}\left(\left[a_{i}, b_{i}\right]\right), \gamma_{\tilde{\theta}}\right) \leq K(B+P+\epsilon)+C .
$$

It is clear that $\left|a_{i}-b_{i}\right| \rightarrow+\infty$ as $i \rightarrow+\infty$. Let $D \subset \tilde{M}$ be a closed ball centered at $\gamma_{\tilde{\theta}}(0)$ with radius $2 K(B+\epsilon+P)+2 C$. We have iterates $T^{k_{i}}$ of the covering isometry $T$ such that $T^{k_{i}}\left(\gamma_{i}\left(\frac{b_{i}-a_{i}}{2}\right)\right)$ belongs to $D$. Since $T$ is an isometry preserving $\gamma_{\tilde{\theta}}$, the lifts $T^{k_{i}}\left(\gamma_{i}\left(\left[a_{i}, b_{i}\right]\right)\right)$ of $\gamma_{\theta}$ remain within a distance of $2 K(B+P+\epsilon)+2 C$ from $\gamma_{\tilde{\theta}}$.

Hence, we get on the one hand a convergent subsequence of the lifts $T^{k_{i}}\left(\gamma_{i}\left(\left[a_{i}, b_{i}\right]\right)\right)$ which converges to a $\tilde{g}$-geodesic $\eta$ that is bi-asymptotic to $\gamma_{\tilde{\theta}}$. The geodesic $\eta$ is a lift of $\gamma_{\theta}$, and hence it is not difficult to show (see Lemma 1.3 in [89]) that $\eta$ has to be an axis of $T$.

On the other hand, since the number of lifts of $\gamma_{\theta}$ meeting the compact set $D$ has to be finite, we have in fact that the number of geodesics $T^{k_{i}}\left(\gamma_{i}\left(\left[a_{i}, b_{i}\right]\right)\right)$ is finite. Therefore, the convergent subsequence of the geodesics $T^{k_{i}}\left(\gamma_{i}\left(\left[a_{i}, b_{i}\right]\right)\right)$ must coincide with its limit $\eta$, and this yields 
that all the $\gamma_{i}$ are bi-asymptotic to $\gamma_{\tilde{\theta}}$ and hence axes of $T$. This leaves us in shape to apply Lemma 8.5 to conclude that the sequence $\beta_{n_{i}}$ must approach both $\gamma_{\tilde{\theta}}$ and $T_{b}\left(\gamma_{\tilde{\theta}}\right)$, that is forbidden by the assumptions in the sequence $\beta_{n_{i}}$.

The contradiction arose from the uniqueness of the lift $\gamma_{i} \epsilon$-approached by $\beta_{n_{i}}$, so we conclude that there must be at least two different lifts of $\gamma_{\theta}$ approached by any subsequence of the geodesics $\beta_{n}$ as $n \rightarrow+\infty$, as we wished to show.

Claim 3 together with Corollary 8.1 imply that for $n$ big enough, we see at least two different lifts $\gamma_{1}^{n}, \gamma_{2}^{n}$ of $\gamma_{\theta}$ which are $\epsilon$-approached by $\beta_{n}$ (in the sense of Definition 8.3 along intervals $\left[c_{j_{1}}^{n}, d_{j_{1}}^{n}\right],\left[c_{j_{2}}^{n}, d_{j_{2}}^{n}\right]$, such that

1. $\lim _{n \rightarrow+\infty}\left|c_{j_{i}}^{n}-d_{j_{i}}^{n}\right|=\infty$ for $i=1,2$.

2. $d_{H}\left(\beta_{n}\left(\left[c_{j_{i}}^{n}, d_{j_{i}}^{n}\right]\right), \gamma_{i}^{n}\right) \leq \epsilon$ for $i=1,2$.

3. $d_{j_{1}}^{n}<c_{j_{2}}^{n}$, and there exists a constant $E>0$ such that $\left|c_{j_{2}}^{n}-d_{j_{1}}^{n}\right| \leq E$.

Let $F_{n}$ be a sequence of covering isometries which send $\gamma_{1}^{n}$ to $\gamma_{\tilde{\theta}}$, in such a way that

$$
d\left(\beta_{n}\left(d_{j_{1}}^{n}\right), \gamma_{\tilde{\theta}}\left(\left[0, l_{g}\left(\gamma_{\theta}\right)\right]\right)\right) \leq \epsilon,
$$

where $l_{g}\left(\gamma_{\theta}\right)$ is the period of $\gamma_{\theta}$. This gives $\tilde{g}_{\delta}$-geodesics $\bar{\beta}_{n}=F_{n}\left(\beta_{n}\right)$ and intervals $\left[s_{1}^{n}, r_{1}^{n}\right],\left[s_{2}^{n}, r_{2}^{n}\right]$ such that

1. $\lim _{n \rightarrow+\infty}\left|r_{i}^{n}-s_{i}^{n}\right|=\infty$ for $i=1,2$.

2. $d_{H}\left(\bar{\beta}_{n}\left(\left[s_{1}^{n}, r_{1}^{n}\right]\right), \gamma_{\tilde{\theta}}\right) \leq \epsilon$.

3. $r_{1}^{n}<s_{2}^{n}$, and there exists a constant $E>0$ such that $\left|s_{2}^{n}-r_{1}^{n}\right| \leq E$.

4. The curve $\bar{\beta}_{n}\left(\left[r_{1}^{n}, s_{2}^{n}\right]\right)$ is contained in the ball $W$ of radius $l_{g}\left(\gamma_{\theta}\right)+$ $\epsilon+E$ centered at $\gamma_{\tilde{\theta}}(0)$ for every large $n$.

5. There exists a lift $\bar{\gamma}_{n}$ of $\gamma_{\theta}$ that is different from $\gamma_{\tilde{\theta}}$ such that $d_{H}\left(\bar{\beta}_{n}\left(\left[s_{2}^{n}, r_{2}^{n}\right]\right), \bar{\gamma}_{n}\right) \leq \epsilon$.

Notice that the number of lifts $\bar{\gamma}_{n}$ has to be finite by their choice, since all of them have points within a distance of $l_{g}\left(\gamma_{\theta}\right)+2 \epsilon+E$ from $\gamma_{\tilde{\theta}}(0)$. So letting $n \rightarrow+\infty$, we get a convergent subsequence of the geodesics $\bar{\beta}_{n}$ which converges to a globally minimizing $\tilde{g}_{\delta}$-geodesic $\beta$. The geodesic $\beta$ is $\epsilon$-close to $\gamma_{\tilde{\theta}}$ in the past and gets $\epsilon$-close to some lift $\eta$ of $\gamma_{\theta}$ in the future. The lift $\eta$ is different from $\gamma_{\tilde{\theta}}$, and replacing $L$ by $\epsilon$ in inequality (7.1) in the proof of Lemma 7.4 we get that in fact

$$
\begin{aligned}
& \lim _{t \rightarrow-\infty} d\left(\beta(t), \gamma_{\tilde{\theta}}\right)=0, \\
& \lim _{t \rightarrow+\infty} d(\beta(t), \eta)=0,
\end{aligned}
$$

thus proving Proposition 8.2. 


\section{Proof of Proposition 8.1}

Item (1) and item (2), (a) of Proposition 8.1 follow from the construction of the metric $g_{\delta}$ (Lemma 8.1) and Proposition 8.2. Item 2, (b) follows from the fact that the limit $\beta$ of the $\epsilon, \tilde{\theta}$-critical geodesics $\bar{\beta}_{n}$ is $\epsilon, \tilde{\theta}$-critical too, together with Proposition 8.2.

\subsection{Morse homoclinic geodesics and bounded asymptote}

To distinguish between general homoclinic geodesics and the sort of homoclinic geodesics found by Morse using variational calculus, we shall denote the latter ones by Morse homoclinic geodesics. Namely, $\beta \subset M$ is Morse homoclinic to a closed geodesic $\gamma$ if there exist two different bi-asymptotic lifts $\alpha_{1}, \alpha_{2}$ in $\tilde{M}$ of $\gamma$ and a lift $\beta_{1}$ of $\beta$ such that $\beta_{1}$ is backward asymptotic to $\alpha_{1}$ and forward asymptotic to $\alpha_{2}$. The best we can say about the asymptotic geometry of the lifts $\gamma_{\tilde{\theta}}, \eta$ of the closed geodesic $\gamma_{\theta}$ in Proposition 8.1 is the following:

Lemma 8.6. Let $(M, g)$ be a compact manifold without conjugate points such that the universal covering is quasi-convex. Let $\gamma_{\tilde{\sigma}} \subset \tilde{M}$, and let $\gamma_{\tilde{\eta}} \in \tilde{M}$ be any geodesic such that

$$
\lim _{t \rightarrow+\infty} \angle\left(\gamma_{\tilde{\eta}}^{\prime}(t),-\nabla b^{\tilde{\sigma}}\left(\gamma_{\tilde{\eta}}(t)\right)\right)=0
$$

where $b^{\tilde{\sigma}}$ is the Busemann function of $\gamma_{\tilde{\sigma}}$. Then

$$
\left.\lim _{t \rightarrow+\infty} \frac{1}{t} d\left(\gamma_{\tilde{\sigma}}, \gamma_{\tilde{\eta}}(t)\right)\right)=0
$$

Proof. Let $K, C$ be the quasi-convexity constants of $(\tilde{M}, \tilde{g})$. To shorten notation, let us call $\beta(t)=\gamma_{\tilde{\eta}}(t)$. Given $n \in \mathbb{N}$, let $t_{n} \rightarrow+\infty$ such that

$$
d\left(\beta(t), \gamma_{-\nabla b^{\tilde{\sigma}}(\beta(t))}\right) \leq \frac{1}{n},
$$

for every $t \in\left[t_{n}, t_{n+1}\right]$. By the continuity of geodesics with respect to initial conditions, we have that $\lim _{n \rightarrow+\infty}\left|t_{n+1}-t_{n}\right| \rightarrow+\infty$. Let $\beta_{n}(t)$ be the Busemann asymptote of $\gamma_{\tilde{\sigma}}$ such that

1. $\beta_{n}(0) \in H_{\tilde{\sigma}}(0)$,

2. There exists $a_{n}>0$ such that $\beta_{n}\left(a_{n}\right)=\beta\left(t_{n}\right)$.

Let $b_{n}>0$ be the supremum of the values of $t>a_{n}$ for which $d\left(\beta, \beta_{n}(t)\right) \leq \frac{1}{n}$. Then we get

1. $d\left(\beta_{n}\left(b_{n}\right), \beta_{n+1}\left(a_{n+1}\right)\right) \leq \frac{1}{n}$, 
2. $d\left(\beta_{n}\left(b_{n}+t\right), \beta_{n+1}\left(a_{n+1}+t\right)\right) \leq K \frac{1}{n}+C$ for every $t \geq 0$.

3. $\lim _{n \rightarrow+\infty}\left|b_{n}-a_{n}\right|=+\infty$.

Item (2) is a consequence of quasi-convexity and the weak asymptoticity. Item (3) follows from the triangular inequality: we have that

$$
|| t_{n+1}-t_{n}|-| b_{n}-a_{n}|| \leq \frac{1}{n}
$$

for every $n>0$, and since $\left|t_{n+1}-t_{n}\right| \rightarrow+\infty$ the same happens to $\left|b_{n}-a_{n}\right|$.

The above properties of $\beta_{n}$ imply that for every $t \geq a_{n}$ and $m>0$,

$$
d\left(\beta_{n}(t), \beta_{n+m}\right) \leq m\left(\frac{K}{n}+C\right),
$$

and hence

$$
\begin{aligned}
d\left(\gamma_{\tilde{\sigma}}(t), \beta_{n+1}(t)\right) & \leq d\left(\gamma_{\tilde{\sigma}}(t), \beta_{1}(t)\right)+n\left(\frac{K}{n}+C\right) \\
& \leq K\left(d\left(\gamma_{\tilde{\sigma}}(0), \beta_{1}(0)\right)+C+n\left(\frac{K}{n}+C\right)\right. \\
& \leq K(D+1)+C(n+1),
\end{aligned}
$$

for every $n>0$, where $D=d\left(\gamma_{\tilde{\sigma}}(0), \beta_{1}(0)\right)$.

Claim: $\quad \lim _{n \rightarrow+\infty} \frac{n}{t_{n}}=0$.

To see this, we can write $t_{n+1}=t_{0}+\sum_{i=0}^{n}\left(t_{n+1}-t_{n}\right)$, and thus

$$
\frac{n}{t_{n}}=\frac{n}{t_{0}+\sum_{i=0}^{n}\left(t_{n+1}-t_{n}\right)} \text {. }
$$

Now, recall that $\left(t_{n+1}-t_{n}\right) \rightarrow+\infty$, so it is an easy exercise to check the claim.

From the claim, we deduce that

$$
\lim _{n \rightarrow+\infty} \frac{1}{t_{n+1}} d\left(\gamma_{\tilde{\sigma}}\left(t_{n+1}\right), \beta_{n}\left(t_{n+1}\right)\right) \leq \lim _{n \rightarrow+\infty} \frac{K(D+1)}{t_{n+1}}+C \frac{n+1}{t_{n+1}}=0,
$$

and by quasi-convexity we obtain

$$
\lim _{n \rightarrow+\infty} \frac{1}{t} d\left(\gamma_{\tilde{\sigma}}(t), \beta_{n}(t)\right)=0 .
$$

Therefore we have for $t \in\left[t_{n}, t_{n+1}\right]$,

$$
d\left(\beta(t), \gamma_{\tilde{\sigma}}(t)\right) \leq d\left(\beta(t), \beta_{n}\right)+d\left(\beta_{n}, \gamma_{\tilde{\sigma}}\right) \leq \frac{1}{n}+d\left(\beta_{n}(t), \gamma_{\tilde{\sigma}}(t)\right),
$$

from which the lemma follows. 
At this point, the bounded asymptote hypothesis appears in the argument for the first time. Notice that the bounded asymptote hypothesis implies quasi-convexity. Item (2b) of Proposition 8.1 tells us that the geodesic $\beta$ behaves asymptotically like an integral orbit of the Busemann flow of $\gamma_{\tilde{\theta}}$, and since $\beta$ is asymptotic to $\eta$ the same holds for $\eta$. But Knieper showed ([60], see also [89]) that under the assumption of bounded asymptote the distance between any two geodesics in $(M, g)$ either diverges at least linearly or the geodesics are asymptotic. Hence, $\eta$ has to be asymptotic to $\gamma_{\tilde{\theta}}$. Now, as in Claim 3 of the proof of Proposition 8.2, we have that a lift of $\gamma_{\theta}$ that is asymptotic to $\gamma_{\tilde{\theta}}$ has to be an axis of the covering isometry $T_{\gamma_{\tilde{\theta}}}$. So we get from Proposition 8.1 (compare with Proposition 5.1 of [89]):

Corollary 8.2. Let $(M, g)$ be a compact Riemannian manifold without conjugate points and bounded asymptote. Assume that there exists a free homotopy class $[\gamma]$ represented by a closed geodesic $\gamma$ such that centralizer of $T=T_{\bar{\gamma}}$ contains a subgroup isomorphic to $\mathbb{Z} \times \mathbb{Z}$, where $\bar{\gamma}$ is a lift of $\gamma$. Then, given $\delta>0$ small and $k>0$, there exists a $\delta$ - $C^{k}$ perturbation $\left(M, g_{\delta}\right)$ of $(M, g)$ satisfying the following properties:

1. The geodesic $\gamma$ is a $g_{\delta}$ geodesic that is a waist in its homotopy class with respect to the $g_{\delta}$-length.

2. There exists a $g_{\delta}$-geodesic $\beta$ that is Morse homoclinic to $\gamma$.

\section{Bounded asymptote and Preissmann's property}

In this section we show Theorem 8.1. One of the crucial steps to show the conjecture in nonpositive curvature manifolds, namely, the existence of homoclinic and connecting orbits in perturbations of flat metrics in the two torus, relies strongly in two key facts: the geometry of nonpositive curvature and two dimensional topology. In nonpositive curvature, we have flat planes in the universal covering if it is not a Gromov hyperbolic space. Moreover, we get flat tori in the manifold if there exists a subgroup of the fundamental group isomorphic to $\mathbb{Z} \times \mathbb{Z}$. The possibility of perturbing the metric keeping a perturbation of a flat torus totally geodesic, allows us to use the theory of minimizing geodesics of surfaces (Morse-Hedlund) to get homoclinic geodesics; as well as to use Mather's theorem about connecting orbits of twist maps. But, if we drop the assumption on the sign of the curvature of the manifold it is not clear whether we can produce chaotic behavior of geodesics by using the geometry of minimizing geodesics of the two torus. In general, it is not known (and seems to be very difficult to prove) if a compact manifold without conjugate points whose universal 
covering is not Gromov hyperbolic has a flat plane in its universal covering. Moreover, it is not known if the existence of a subgroup of the fundamental group isomorphic to $\mathbb{Z} \times \mathbb{Z}$ implies that the manifold has an immersed, flat torus. There are some examples of manifolds without conjugate points where the flat strip theorem is false [19].

Theorem 8.1 is a generalization of Theorem 7.3, and shows that the same weaker version of the conjecture that holds for manifolds of nonpositive curvature holds for manifolds with bounded asymptote. The main idea of the proof is the existence of homoclinic geodesics in perturbations of metrics without conjugate points whose fundamental group contains a subgroup isomorphic to $\mathbb{Z} \times \mathbb{Z}$.

\subsection{Shadowing of geodesics and the Preissmann property in bounded asymptote}

The proof of the fact that $\epsilon-C^{k}$-shadowing property implies Preissmann's property, for $\epsilon$ as in the statement of Theorem 8.1, is a natural generalization of the argument used in the proof of Theorems 7.2, 7.3. Let us give a brief sketch of the proof (see [89] for details). Suppose that there is a subgroup $S$ of $\pi_{1}(M)$ isomorphic to $\mathbb{Z} \times \mathbb{Z}$. Let $T, P$ be two commuting covering isometries. It is easy to show (see for instance [22]) that if $\alpha$ is an axis of $T$, then $P^{n}(\alpha)$ is an axis of $T$ for every $n \in \mathbb{Z}$. As in the proof of Lemma 7.2 in the nonpositive curvature case, we have to find a good pair of generators $T_{1}, T_{2}$ of $S$. Let us make precise this notion.

Definition 8.4. Let $G$ be an abelian, finitely generated subgroup of $\pi_{1}(M)$. We say that a set of generators $\left\{a_{1}, a_{2}, \ldots, a_{m}\right\}$ is tight if

1. $d_{a_{1}}$ is the least displacement among the non-trivial elements of $G$,

2. $d_{a_{i+1}}$ is the least displacement among the elements of the quotient of $G$ by the subgroup generated by $\left\{a_{1}, a_{2}, . ., a_{i}\right\}$, for every $i \leq m$.

It is not difficult to get a tight set of generators of an abelian subgroup of $\pi_{1}(M)$ when $M$ is compact (see for instance [88], [89]). In our case, let $T_{1}, T_{2}$ be a pair of tight generators of $S$, and let us suppose that $T_{1}$ has the minimum displacement among the nontrivial elements of $S$. The special property of tight generators that will be important for the proof of the theorem is the following.

Lemma 8.7. Let $(M, g)$ be a compact manifold without conjugate points and let $S$ be a rank two abelian subgroup of the fundamental group with tight generators $T_{1}, T_{2}$. Let us suppose that $T_{1}$ has the minimum displacement among the nontrivial elements of $S$. Then each two different axes of $T_{1}$ in $\tilde{M}$ are separated by a distance which is bounded below by the constant $C=\frac{1}{3 B} \rho(M)$ (which depends only on the manifold $(M, g)$ ) 
The proof of this lemma is not difficult, we leave the details to the reader. Now, let $\gamma$ be a closed geodesic such that a lift $\gamma_{1}$ of $\gamma$ is an axis of $T_{1}$. Let $\left(M, g_{\delta}\right)$ be a $\delta-C^{k}$ perturbation of $(M, g)$ satisfying Corollary 8.2. Let $\gamma_{2}$ be the other axis of $T_{1}$ such that there is a homoclinic geodesic $\beta \subset \tilde{M}$ that is $\alpha$-asymptotic to $\gamma_{1}$ and $\omega$-asymptotic to $\gamma_{2}$. The argument in the proof of Theorems 7.2, 7.3 can be generalized without problems because,

Claim: Under the assumption of bounded asymptote, there are no homoclinic geodesics in $(\tilde{M}, g)$ "connecting" two different axes of a covering isometry ([89], Lemma 5.2).

Here we denote by $(\tilde{M}, g)$ the universal covering endowed with the pullback of $g$. In fact, the bounded asymptote hypothesis implies that given $\tilde{\gamma}$, $\tilde{\beta}$ two asymptotic geodesics in $\tilde{M}$, with $\tilde{\gamma}(0), \tilde{\beta}(0)$ in the same horosphere of $\tilde{\gamma}$, then

$$
d(\tilde{\gamma}(t), \tilde{\beta}(t)) \leq B d(\tilde{\gamma}(0), \tilde{\beta}(0)),
$$

for every $t \geq 0$. So every geodesic that is asymptotic to $\gamma_{1}$ and whose initial conditions are close to $\gamma_{1}$ must remain in a tubular neighborhood of $\gamma_{1}$ of small diameter.

At this point, we used the bounded asymptote condition in the argument for the second time. Therefore, it is not difficult to show that if we can $\rho-C^{k}$ shadow the homoclinic geodesic $\beta$, then its shadow $\bar{\beta}$, that is a $g$ geodesic, must be within a distance of the axes of $T_{1}$ which is comparable to $\rho$. Hence, the distance between the axes must be comparable to $\rho$ as well. So if $\rho$ is suitably small we contradict the fact that the minimum distance between $\gamma_{1}$ and $\gamma_{2}$ is bounded below by some universal constant according to Lemma 8.7. The contradiction yields the proof of the claim.

\subsection{Topological stability and Preissmann property}

The proof of the fact that topological stability implies the Preissmann property (Lemma 5.4 in [89]) is as well a generalization of the argument in Theorems 7.2, 7.3. Let us recall the main idea in the proof of Lemma 7.1: the image of the perturbed geodesic $\beta$, homoclinic to a closed geodesic $\gamma$, by a semiconjugacy of the perturbed geodesic flow with the flat geodesic flow coincides with the image of $\gamma$ by the semiconjugacy. This is a consequence of the fact that the image of $\beta$ by the semiconjugacy has to be a geodesic of the flat metric, and by continuity of the semiconjugacy this geodesic has to be asymptotic to the image of $\gamma$. Now, two asymptotic lines in the plane have to coincide, thus concluding the proof. Under the hypothesis of Theorem 8.1, there is no restriction on the sign of the curvature, so the behavior of bi-asymptotic geodesics might be different from the behavior of straight lines. We have already noticed that the flat strip theorem does not hold in manifolds without conjugate points. But the bounded asymptote 
assumption implies that there are no homoclinic geodesics in $(M, g)$ whose lifts in $\tilde{M}$ "connect" two different axes of a covering isometry (Claim 2.1 in the previous subsection). Hence, the image of $\beta$ by the semiconjugacy must coincide with the image of $\gamma$, and the same argument proceeds to conclude the proof of the theorem.

The issue of the nonexistence of homoclinic behavior in manifolds without conjugate points is a delicate point of the analysis of the relationship between topological stability and the Preissmann property. However, the absence of homoclinic behavior is not essential in the study of the connections between the shadowing property and the Preissmann property. It is not difficult to show that Theorem 8.1 hold under the assumption of $K, 0$ quasi-convexity of the universal covering.

Proposition 8.3. Let $(M, g)$ be a compact manifold without conjugate points whose universal covering is K,0-quasi-convex. Then either the $\epsilon$ $C^{k}$ shadowing property for some appropriate $\epsilon=\epsilon(M)>0$ or the $C^{k}$ topologically stability of the geodesic flow imply the Preissmann property.

We leave the details of the proof to the reader. Notice that the $K, 0$-quasiconvexity is similar to the bounded asymptote condition. In fact, bounded asymptote condition implies $K, 0$-quasi-convexity for some $K>0$, because the bounded asymptote is an assumption of similar type on the norm of stable Jacobi fields (whose integrals give the behavior of Busemann flows). The converse of this statement is likely to be false, however we do not know examples which support this assertion. The ideas developed in this section rise the following interesting question in itself: Are there manifolds without conjugate points with homoclinic geodesics connecting two different axes of a covering transformation? We would guess that the answer to this question is negative, because this type of homoclinic behavior is typical of manifolds with conjugate points. However, we do not know the answer to this question so far. We shall deal with this problem in the forthcoming sections.

\section{$3 \quad$ Further results in certain manifolds without conjugate points}

In this section we briefly discuss the proof of Theorems 8.2 and 8.3 , which are generalizations of Theorem 8.1 under the weaker hypotheses introduced in the chapter. We follow [92]. 


\subsection{Morse homoclinic geodesics in perturbations of metrics without conjugate points}

We follow the strategy used to deal with manifolds with bounded asymptote. So we need first of all a generalization of Corollary 8.2 for this case.

Proposition 8.4. Let $(M, g)$ be a compact three-dimensional manifold without conjugate points such that the universal covering endowed with the pullback of $g$ is a quasi-convex space and satisfies the weak axiom of asymptoticity. Let $\{S, T\}$ be a pair of tight generators of a subgroup of covering isometries which is isomorphic to $\mathbb{Z} \times \mathbb{Z}$. Let $\gamma$ be a closed geodesic with a lift $\gamma_{\theta}$ which is an axis of $T$. Let $\gamma_{\eta} \subset \tilde{M}$ be any lift of $\gamma$ such that

$$
\lim _{t \rightarrow+\infty} \angle\left(\gamma_{\eta}^{\prime}(t),-\nabla b^{\theta}\left(\gamma_{\eta}^{\prime}(t)\right)\right)=0
$$

where $\angle(v, w)$ is the angle in the metric $\tilde{g}$ formed by the vectors $v, w$, and $b^{\theta}$ is the Busemann function of $\gamma_{\theta}$ in $(M, \tilde{g})$. Then $\gamma_{\eta}$ is also an axis of $T$, and in particular, the above geodesics are bi-asymptotic.

Proposition 8.4 implies in particular that the Hausdorff distance between any two lifts of the closed geodesic $\gamma$ given in the proposition is either finite or diverges at least linearly. Let us give an outline of the proof of Proposition 8.4 ([92]). Proposition 8.1 already implies the existence of a pair of lifts of closed geodesics in $(\tilde{M}, \tilde{g})$ such that the distance from one to the other diverges sublinearly. The existence of a subgroup of $\pi_{1}(M)$ isomorphic to $\mathbb{Z} \times \mathbb{Z}$ such that one of the above lifts is an axis of $T$ implies that there exists a grid of axes of $T$. Now, in dimension 3, and with the help of the action of $\pi_{1}(M)$ on the pair of initial lifts of closed geodesics, we show that either such pair of lifts is bi-asymptotic, or we get a sequence of balls in $\tilde{M}$ whose volumes increase at most as a polynomial function of their radii. Then we apply Gromov's theorem relating polynomial growth of the fundamental group with virtually nilpotent groups; Croke-Schröeder theorem [26] connecting nilpotent subgroups of $\pi_{1}(M)$ and abelian subgroups when $M$ is compact and has no conjugate points; and Burago-Ivanov solution of the Hopf conjecture in n-dimensional tori (too many big shots indeed ...). So we conclude that the assumptions on Proposition 8.4 imply that either the pair of lifts in Proposition 8.1 are in fact bi-asymptotic, or the manifold $M$ is a flat torus. Since in a flat metric the divergence of axes in $\tilde{M}$ is at least linear, we conclude that the pair of lifts in Proposition 8.1 had to be bi-asymptotic anyway.

It is possible to prove Proposition 8.4 following a simpler approach based in elementary properties of the fundamental group of compact manifolds without conjugate points. However, the proof would be long and technical and we prefer not to present it in this survey. Although Proposition 8.4 seems to be a mild improvement of Corollary 8.2 (only holds for three 
dimensional manifolds and the assumptions on the global geometry of the universal covering are not the ideal ones), the proof is not easy. Even for three dimensional manifolds, we do not have a version of Proposition 8.1 available without the assumption of quasi-convexity.

\subsection{On the nonexistence of Morse homoclinic geodesics in manifolds without conjugate points}

The second step of the proof of the theorem requires a result about the nonexistence of Morse type homoclinic geodesics in manifolds without conjugate points, like in the proofs of the previous cases. Our main reference is [92]. We get a partial nonexistence result assuming weak asymptoticity and quasi-convexity. In the proof of this result the weak asymptoticity assumption plays once more time a crucial role.

Lemma 8.8. Let $(M, g)$ be a compact manifold without conjugate points satisfying the weak axiom of asymptoticity, such that $(\tilde{M}, g)$ is $K, C$-quasiconvex. Then, if the Haussdorff distance between two different axes $\gamma_{1}, \gamma_{2}$ of a covering isometry is greater than $C$, there are no geodesics $\beta$ in $\tilde{M}$ satisfying

$$
\lim _{t \rightarrow-\infty} d\left(\beta(t), \gamma_{1}\right)=0
$$

and

$$
\lim _{t \rightarrow+\infty} d\left(\beta(t), \gamma_{2}\right)=0
$$

Proof. The proof of this lemma is much simpler than the proof of Proposition 8.4, so we give it in detail for the sake of completeness. Let $p=\gamma_{1}(0)$, $q=\gamma_{2}(0)$, such that the maximum distance between $\gamma_{1}$ and $\gamma_{2}$ is attained at $d(p, q)$. Let $\gamma=\Pi\left(\gamma_{1}\right)$, and let $l_{g}(\gamma)>0$ be the period of $\gamma . l_{g}(\gamma)$ is the period of $\Pi\left(\gamma_{2}\right)$ too, because all minimizing geodesics in the same free homotopy class have the same period. Then the points $p_{n}=\gamma_{1}\left(n l_{g}(\gamma)\right)$, $q_{n}=\gamma_{2}\left(n l_{g}(\gamma)\right)$ satisfy $d\left(p_{n}, q_{n}\right)=d(p, q)$. Assume that $d(p, q)>C$ and suppose that there exists a geodesic $\beta \subset \tilde{M}$ such that

$$
\begin{aligned}
& \lim _{t \rightarrow-\infty} d\left(\beta(t), \gamma_{1}\right)=0, \\
& \lim _{t \rightarrow+\infty} d\left(\beta(t), \gamma_{2}\right)=0 .
\end{aligned}
$$

Given $\epsilon>0$, let $t_{\epsilon}<0, s_{\epsilon}<0$ be such that $d\left(\beta\left(t_{\epsilon}\right), \gamma_{1}\left(s_{\epsilon}\right)\right)<\epsilon$. Since the axes $\gamma_{1}$ and $\gamma_{2}$ are bi-asymptotic, the geodesic $\beta$ is bi-asymptotic to both $\gamma_{1}$ and $\gamma_{2}$. Therefore, by Lemma $4.9, \beta$ is an orbit of the Busemann flow associated to $\gamma_{1}$ and $\gamma_{2}$. This implies that, by definition, the geodesic $\beta$ is the limit of the geodesics $\left[\beta\left(t_{\epsilon}\right), \gamma_{1}(t)\right]$ joining the points $\beta\left(t_{\epsilon}\right), \gamma_{1}(t)$, as 
$t \rightarrow+\infty$. Since $\beta(t)$ approaches $\gamma_{2}$ as $t \rightarrow+\infty$, given $\delta>0$ there exist $t_{0}>0, n_{0}>0$, and points $x_{t, n} \in\left[\beta\left(t_{\epsilon}\right), \gamma_{1}(t)\right]$, such that

$$
d\left(q_{n}, x_{t, n}\right)<\delta
$$

for some $n>n_{0}$ and every $t>t_{0}$. By the quasi-convexity of the metric in $\tilde{M}$ we have

$$
d\left(\left[\beta\left(t_{\epsilon}\right), \gamma_{1}(t)\right], \gamma_{1}\left[s_{\epsilon}, t\right]\right) \leq K d\left(\beta\left(t_{\epsilon}\right), \gamma_{1}\left(s_{\epsilon}\right)\right)+C \leq K \epsilon+C .
$$

By the triangle inequality, we get

$$
d\left(q_{n}, \gamma_{1}\left[s_{\epsilon}, t\right]\right) \leq d\left(q_{n}, x_{t, n}\right)+d\left(x_{t, n}, \gamma_{1}\left[s_{\epsilon}, t\right]\right) \leq \delta+K \epsilon+C,
$$

since $x_{t, n} \in\left[\beta\left(t_{\epsilon}\right), \gamma_{1}(t)\right]$. This clearly implies that the distance between $q_{n}$ and $\gamma_{1}$ cannot be greater than $C$, otherwise we would get

$$
C+a<d\left(q_{n}, \gamma_{1}\right) \leq d\left(q_{n}, \gamma_{1}\left[s_{\epsilon}, t\right]\right) \leq \delta+K \epsilon+C,
$$

for some $a>0$. So $a<K \epsilon+\delta$, but by the choice of $\delta$ and $\epsilon$ these numbers can be arbitrarily small. Therefore, we conclude that if there exists a homoclinic geodesic $\beta$ connecting $\gamma_{1}, \gamma_{2}$ in the way described in the hypothesis, then the distance between $\gamma_{1}$ and $\gamma_{2}$ must be less than $C$, as claimed.

As we have already mentioned, it would be interesting to know if Morse homoclinic behavior is possible in manifolds without conjugate points without any additional assumptions on the metric. In surfaces it is not possible by the work of Morse [69]. In higher dimensions, we can show for instance that

Lemma 8.9. Let $(M, g)$ be a compact manifold without conjugate points such that $\pi_{1}(M)$ has non-trivial center $Z$. Then closed geodesics in a homotopy class in $Z$ cannot have Morse homoclinic geodesics.

Proof. We just give an outline of proof. The proof relies in Lemma 8.5, which implies that any geodesic $\beta$ in $\tilde{M}$ that is backward asymptotic to an axis $\gamma_{1}$ of a covering isometry $T$, and forward asymptotic to another axis $\gamma_{2}$ of $T$, cannot intersect any axis of $T$. On the other hand, if we suppose that $\pi_{1}(M)$ has non-trivial center $Z$, and $T \in Z$ is a covering isometry, we have that $\tilde{M}$ is foliated by the axes of $T$. Thus, any Morse homoclinic geodesic has to intersect some axis, proving that such geodesics cannot exist under the hypothesis of the lemma. 


\subsection{Proof of Theorem 8.2}

As in Chapter 7 we argue by contradiction. Suppose that there exists a subgroup $G$ of $\pi_{1}(M)$ isomorphic to $\mathbb{Z} \times \mathbb{Z}$. Let $S, T$ be tight generators of $G$, and let $\gamma$ be a closed geodesic in $M$ that is a waist in $G$ such that the axes of $T$ are lifts of $\gamma$. Let $\gamma_{1}, \gamma_{2}$ be two different axes of $T$. Lemma 8.7 (or Lemma 5.3 in [86]) implies that the minimum distance from points in $\gamma_{1}$ to $\gamma_{2}$ is at least $\frac{\rho(M)}{2}$, where $\rho(M)$ is the injectivity radius of $M$.

Applying Proposition 8.1 and Proposition 8.4, given $\delta>0$ we get a $\delta$ - $C^{k}$ perturbation $\left(M, g_{\delta}\right)$ of $(M, g)$, for any $k>0$, such that $\gamma$ is a $g_{\delta}$-waist in its homotopy class and there are lifts $\gamma_{1}, \gamma_{2}$ of $\gamma$ which are axes of the same covering isometry $T$, connected by a Morse homoclinic $\widetilde{g_{\delta}}$-geodesic $\beta$. We can suppose that $\beta(t)$ approaches $\gamma_{1}$ when $t \rightarrow-\infty$, and approaches $\gamma_{2}$ as $t \rightarrow+\infty$. Suppose that $\beta$ is $\epsilon$-shadowed by some $g$-geodesic $\bar{\beta}$ in $\tilde{M}$. Let us consider $n<0, s_{n}<0$ such that

$$
d\left(\gamma_{1}(n), \beta\left(s_{n}\right)\right) \leq \frac{1}{|n|},
$$

where $d$ is the distance in $(\tilde{M}, \tilde{g})$. Let $a_{n}$ be such that $d\left(\beta\left(s_{n}\right), \bar{\beta}\left(a_{n}\right)\right) \leq \epsilon$. Then we have that

$$
d\left(\gamma_{1}, \bar{\beta}\left(a_{n}\right)\right) \leq d\left(\gamma_{1}, \beta\left(s_{n}\right)\right)+d\left(\beta\left(s_{n}\right), \bar{\beta}\left(a_{n}\right)\right) \leq \epsilon+\frac{1}{|n|} .
$$

Since $\bar{\beta}$ is a $\tilde{g}$-geodesic which is clearly asymptotic to both $\gamma_{1}$ and $\gamma_{2}$, by the weak axiom of asymptoticity (Lemma $4.9(2)) \bar{\beta}$ is a limit of $\tilde{g}$-geodesics $\bar{\beta}_{n, m}$ of the type

$$
\bar{\beta}_{n, m}=\left[\bar{\beta}\left(a_{n}\right), \gamma_{1}\left(T_{m}\right)\right],
$$

where $T_{m} \rightarrow+\infty$, and the notation $[x, y]$ designates the (unique) $\tilde{g}$-geodesic curve joining $x, y$. By the $K, C$-quasi-convexity we get

$$
d\left(\gamma_{1}, \bar{\beta}\right) \leq K\left(\epsilon+\frac{1}{|n|}\right)+C
$$

and since $|n|$ can be taken arbitrarily large, we conclude

$$
d\left(\gamma_{1}, \bar{\beta}\right) \leq K(\epsilon)+C .
$$

The same estimate holds for the distance between $\bar{\beta}$ and $\gamma_{2}$. Thus we get

$$
d\left(\gamma_{1}, \gamma_{2}\right) \leq 2 K \epsilon+2 C,
$$

so by the claim we obtain

$$
\frac{\rho(M)}{2}<d\left(\gamma_{1}, \gamma_{2}\right) \leq 2 K \epsilon+2 C
$$


This yields

$$
\frac{1}{2 K}\left(\frac{\rho(M)}{2}-2 C\right) \leq \epsilon .
$$

This estimate determines the constants $C, \epsilon$ in the statement of the theorem, in terms of $K$ and the injectivity radius. For if we take $C<\frac{\rho(M)}{4}$, the left hand side of the inequality is strictly positive, and if we consider any $\epsilon<\frac{1}{2 K}\left(\frac{\rho(M)}{2}-2 C\right)$ we get a contradiction. Since the contradiction arises from the existence of an abelian subgroup of $\pi_{1}(M)$ isomorphic to $\mathbb{Z} \times \mathbb{Z}$ together with the $\epsilon$-shadowing assumption, we get that $\pi_{1}(M)$ must enjoy the Preissmann's property provided that the geodesic flow has the $\epsilon-C^{k}$ shadowing property for some $k>0$ and $\epsilon<\frac{1}{2 K}\left(\frac{\rho(M)}{2}-2 C\right)$.

\subsection{Proof of Theorem 8.3}

We extend the argument of Chapter 7 to this case. If there are no Morse type homoclinic geodesics in $(M, g)$ whose lifts in $\tilde{M}$ are asymptotic to two different axes of a covering isometry, we get the Preissmann's property. However, Lemma 8.8 just tells us that there are no such geodesics if the Hausdorff distance between the axes of a covering isometry is greater than $C$, the quasi-convex constant. So in principle, a pair of axes in $\tilde{M}$ which are close to each other might be connected by lifts of Morse type homoclinic geodesics.

At this point we use the strong stability assumption. Assume by contradiction that there is a subgroup $S$ of $\pi_{1}(M)$ isomorphic to $\mathbb{Z} \times \mathbb{Z}$. Let $\gamma$ be a closed geodesic whose homotopy class belongs to $S$ and whose length is the shortest among closed loops with homotopy class in $S$. By the proof of Theorem 8.2, the distance between two different lifts $\tau, \sigma$ of $\gamma$ which are axes of a covering isometry $T$ is at least $\frac{\rho(M)}{2}$. So by Lemma 8.8 and the hypothesis of Theorem 8.3, there are no Morse type homoclinic geodesics in $(M, g)$ whose lifts connect $\tau, \sigma$. Now, apply Proposition 8.1 and Proposition 8.4 to get arbitrarily small perturbations $(M, \bar{g})$ of $(M, g)$ in the $C^{k}$ topology such that $\gamma$ is a waist in its homotopy class with respect to $\bar{g}$ length, and such that there is a homoclinic geodesic $\bar{\beta}_{0} \subset M$ with a lift $\bar{\beta} \subset \tilde{M}$ connecting two different axes $\gamma_{1}, \gamma_{2}$ of $T$. Let $f$ be the equivalence between orbits of the geodesic flows of $(M, \bar{g})$ and $(M, g)$. By the continuity of $f$, the image $\beta_{0}=f\left(\bar{\beta}_{0}\right)$ is a $g$-geodesic that is homoclinic to $f(\gamma)$. By lifting $f$ to

$$
\tilde{f}: T_{1}(\tilde{M}, \bar{g}) \longrightarrow T_{1}(M, \tilde{g})
$$

$f\left(\bar{\beta}_{0}\right)$ lifts in $\tilde{M}$ to a $\tilde{g}$-geodesic $\beta$ that connects two axes $\alpha_{1}, \alpha_{2}$ of a covering isometry $T^{\prime}$. These axes are lifts of $f(\gamma)$, moreover, $\tilde{f}\left(\gamma_{1}\right)=\alpha_{1}$, and $\tilde{f}\left(\gamma_{2}\right)=\alpha_{2}$. 
The natural homomorphism

$$
f^{*}: \pi_{1}(M) \longrightarrow \pi_{1}(M)
$$

induced by $f$ in homotopy satisfies the following property: if $G$ is a covering isometry of $(\tilde{M}, \bar{g})$, and $c$ is a closed $\bar{g}$-geodesic with nontrivial homotopy class, then

$$
\tilde{f}(G(\tilde{c}))=f^{*}(G)(\tilde{f}(\tilde{c})),
$$

for every lift $\tilde{c}$ of $c$ in $(\tilde{M}, \bar{g})$ (here we identify covering isometries with elements of the homotopy group of $M$ ).

Now, by hypothesis, $f^{*}$ acts trivially in the free homotopy group, and therefore the homotopy class of $f(\gamma)$ is the same as the homotopy class of $\gamma$. So $T=T^{\prime}, f(\gamma)$ is a closed geodesic of minimal $g$-length among the closed loops with homotopy class in $S$; and by Lemma 8.8, there are no homoclinic $g$-geodesics connecting any two different lifts of $f(\gamma)$ in $\tilde{M}$. Hence, $\alpha_{1}$ and $\alpha_{2}$ coincide, and $\tilde{f}(\bar{\beta})$ is bi-asymptotic to $\tilde{f}\left(\gamma_{1}\right)=\alpha_{1}=\alpha_{2}=\tilde{f}\left(\gamma_{2}\right)$. On the other hand, by the contradiction assumption we have that there exists a nontrivial covering isometry $P$ in the subgroup $S$ such that

1. $P\left(\gamma_{2}\right)=\gamma_{1}$,

2. $P$ does not belong to the subgroup generated by $T$.

Since the action of $f^{*}$ in $\pi_{1}(M)$ is trivial, we must have that

$$
\tilde{f}\left(\gamma_{2}\right)=\tilde{f}\left(P\left(\gamma_{1}\right)\right)=f^{*}(P)\left(\tilde{f}\left(\gamma_{1}\right)\right)=P\left(\tilde{f}\left(\gamma_{1}\right)\right) .
$$

Hence, since we have that $\tilde{f}\left(\gamma_{1}\right)=\tilde{f}\left(\gamma_{2}\right)$ we get that

$$
P\left(\tilde{f}\left(\gamma_{1}\right)\right)=\tilde{f}\left(\gamma_{1}\right),
$$

namely, the isometry $P$ preserves the axis $\tilde{f}\left(\gamma_{1}\right)$. This implies that $P$ must belong to the subgroup generated by $T$, leading to a contradiction. The contradiction arose from the existence of a subgroup of $\pi_{1}(M)$ isomorphic to $\mathbb{Z} \times \mathbb{Z}$, thus finishing the proof of the Theorem.

\section{Weak stability and Thurston's geometriza- tion conjecture}

We would like to finish the survey with a brief discussion about Thurston's geometrization conjecture and its connections with the weak stability conjectures stated in the introduction of Chapter 7. Our main references for this subject are [96], [99], [56]. The term geometrization means the existence of a geometric structure for a manifold. The term geometry in this context is a model, simply connected Riemannian manifold $X$ which is a 
homogeneous space. A manifold $M$ admits a geometric structure if it admits a locally homogeneous metric, which is equivalent to say that $M$ is homeomorphic to the quotient of a homogeneous, simply connected space $X$ by the action of a discrete subgroup of isometries of $X$.

\subsection{The Poincaré conjecture}

Surfaces admit geometric structures by the well known theory of classification of surfaces: two-dimensional spaces are quotients of either the two sphere, the Euclidean plane or the hyperbolic plane by discrete subgroup of isometries. However, in higher dimensions the classification theory of manifolds is much harder, and in the late 1970's Thurston proposed what it would be the complete list of geometric structures on three-dimensional manifolds.

The first geometrization problem in the realm of three-dimensional manifolds is the so-called Poincaré conjecture, which claims that a compact, simply connected three-dimensional manifold is homeomorphic to $S^{3}$. Poincaré proposed a proof which had a gap, and since then this problem has fascinated mathematicians and inspired a great body of work in topology.

The conjecture in dimension 4 was proved by Freedman [39] in 1982, in dimension 5 by Zeeman [104] (1961), in dimension 6 was shown by Stallings [98] (1962), and for dimension higher than 6 by Smale [97] in 1961. Actually, Smale extended later his proof to include all dimensions $n \geq 5$.

The recent work of Perelman [74] (2003), certified by many mathematicians around the world (Kleiner-Lott [56], Besson [14] and many others) seems to have solved the Poincare conjecture. The work of Perelman is remarkable not only because of the solution of the Poincaré conjecture, but also because it would lead to the solution of the Thurston's geometrization conjecture (see [56] for instance).

\subsection{Thurston's geometrization conjecture}

Before stating Thurston's geometrization conjecture we need to recall some basic notions of the theory of three-dimensional manifolds. We won't enter into deep detail about the theory of three-dimensional manifolds because this subject is in itself an incredibly huge area of topology. Threedimensional manifolds possess what is known as a standard two-level decomposition. First of all, there is the connected sum decomposition proved by Kneser [59], which says that every compact three-manifold is the connected sum of a finite collection of prime three-manifolds. A three-manifold 
$M$ is called prime if whenever we can express $M$ as a connected sum $M_{1} \sharp M_{2}$, then either $M_{1}$ or $M_{2}$ is homeomorphic to $S^{3}$. Milnor [68] showed that when $M$ is orientable, this connected sum decomposition is unique.

The second kind of decomposition of three-manifolds is the so-called Jaco-Shalen-Johannson torus decomposition [55], [54], which states that irreducible orientable compact three-manifolds have a canonical (up to isotopy) minimal collection of disjointly embedded, two-sided incompressible tori such that if we remove these tori from the manifold then each remaining connected component is either atoroidal or a Seifert fiber space. A surface $S \subset M$ in a three-dimensional manifold $M$ is called incompressible if $S$ is neither $S^{2}$ nor the projective space, and the natural inclusion of $\pi_{1}(S)$ into $\pi_{1}(M)$ is injective. A three-manifold is called atoroidal if every incompressible torus is isotopic to an incompressible boundary component of the manifold. Of course, if the manifold has no boundary, the above definition implies that there is no incompressible torus in the manifold. A three-manifold $M$ is called a Seifert fiber space if $M$ is foliated (fibred) by circles (the fibers) in a way that each point of $M$ has an open neighborhood which is homeomorphic to either a fibred solid torus or a fibred solid Klein bottle. The unit tangent bundle of a surface is an example of Seifert fiber space. A famous result of Epstein [36] shows that every compact three-dimensional manifold that is foliated by circles is a Seifert fiber space, so we can take as definition of compact Seifert fiber space a compact three-dimensional manifold foliated by circles.

After all these preliminaries, we can state Thurston's geometrization conjecture:

Conjecture: Let $M$ be a prime three-manifold that is either atoroidal or a Seifert fiber space. Then $M_{0}$ has exactly one of the following geometric structures:

1. Euclidean geometry,

2. Hyperbolic geometry,

3. Spherical geometry,

4. The geometry of $S^{2} \times \mathbb{R}$,

5. The geometry of $H^{2} \times \mathbb{R}$,

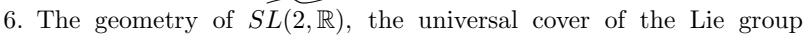
$S L(2, \mathbb{R})$, of $2 \times 2$ matrices with determinant 1 , 
7. Nil geometry, where Nil is the real, three-dimensional group of upper triangular matrices with only 1 in the diagonal, under matrix multiplication,

8. Sol geometry.

The Nil group is the three-dimensional Heisenberg group of nilpotent matrices. The Sol group is most easily defined as a split extension of $\mathbb{R}^{2}$ by $\mathbb{R}$. We have the following exact sequence

$$
0 \longrightarrow \mathbb{R}^{2} \longrightarrow S o l \longrightarrow \mathbb{R} \longrightarrow 0,
$$

where an element $t \in \mathbb{R}$ acts in $\mathbb{R}^{2}$ by the hyperbolic map $f_{t}(x, y)=$ $\left(e^{t} x, e^{-t} y\right)$. For instance, torus bundles over the circle with hyperbolic gluing maps admit Sol geometric structures.

\subsection{Applications of the Thurston's geometrization con- jecture to weak stability problems}

As we mentioned before, Perelman's work about the Poincaré conjecture seems very promising regarding the solution of Thurston's geometrization conjecture. So we would like to state some consequences of Thurston's conjecture combined with the results of Chapters $5,6,7,8$. We shall adopt the notation of topologists to distinguish compact manifolds from compact manifolds without boundary, which we shall denote by closed manifolds. Our main result would be the following:

Theorem 8.4. Let $(M, g)$ be a closed, prime, three-dimensional manifold without conjugate points. Assume that Thurston's geometrization conjecture is true and that $M$ satisfies one of the following conditions:

1. The geodesic flow is expansive,

2. $M$ has bounded asymptote and the geodesic flow is $C^{k}$-topologically stable for some $k \in \mathbb{R}$,

3. $M$ has bounded asymptote and the geodesic flow satisfies the $\epsilon-C^{k}$ shadowing property for $\epsilon=\frac{1}{6 B} \rho(M)$, where $\rho(M)$ is the injectivity radius of $M$.

Then $M$ admits a hyperbolic metric of constant curvature -1 .

Theorem 8.4 solves the conjectures posed in the Introduction of Chapter 7 for prime, three-dimensional manifolds of bounded asymptote: the existence of a hyperbolic structure implies that the fundamental group is Gromov hyperbolic. A slightly more general version of Theorem 8.4 can be stated using the assumptions of Theorems 8.2 and 8.3 . 
The proof of Theorem 8.4 relies in the hyperbolic properties of the fundamental group of $M$ proven in Chapters $6,7,8$, under the hypotheses of the theorem. The proof of item (1) is perhaps the simplest one. By Theorem 6.12 , the expansiveness of the geodesic flow implies that the fundamental group is Gromov hyperbolic. In particular, the fundamental group has the Preissmann property and therefore, the manifold is atoroidal. So we can apply Thurston's geometrization conjecture to conclude that $M$ admits one of the geometric structures in the above list. Since $\pi_{1}(M)$ is Gromov hyperbolic, the universal covering $(\tilde{M}, \tilde{g})$ is Gromov hyperbolic. Hence, by the results of Chapter 6 , any metric $h$ in $M$ has the property that $(\tilde{M}, \tilde{h})$ is Gromov hyperbolic. So the geometric structure of $M$ has to be a Gromov hyperbolic space, and the only one with this property is the hyperbolic three-space.

The proofs of items (2), (3) rely as well in the Preissmann property, which holds under the assumptions of Theorem 8.4 by Theorem 8.1. So the following partial answer to a question posed in Chapter 6 would be enough to finish the proof of the theorem:

Theorem 8.5. Assume that Thurston's geometrization conjecture is true. Let $(M, g)$ be a closed, prime, three-dimensional manifold without conjugate points whose fundamental group has the Preissmann property. Then $M$ admits a hyperbolic geometric structure.

The proof of Theorem 8.5 requires a certain amount of work, so we just give an outline of the argument and leave the details to the reader.

1) First of all, if $M$ has no conjugate points then it cannot admit a geometric structure whose model space is either $S^{3}, S^{2} \times \mathbb{R}$. This is because $\tilde{M}$ is diffeomorphic to $\mathbb{R}^{3}$ and none of the above geometries satisfies this property.

2) By Theorem 6.1, if $M$ admits a $N i l$ geometric structure, then $\pi_{1}(M)$ would be nilpotent and hence abelian. Since $M$ is three-dimensional and is an Eilenberg-Maclane space, the rank of $\pi_{1}(M)$ as an abelian group has to be 3 (see for instance [60]). Thus, $\pi_{1}(M)$ is not Preissmann. The same conclusion follows if we suppose $(M, g)$ analytic and having a $S o l$ structure: Theorem 6.1 would imply that $\pi_{1}(M)$ has an abelian subgroup of finite index, which has to have rank 3 contradicting the Preissmann property. However, since we are not assuming analyticity for $(M, g)$ we have to deal with Sol using a different argument.

3) The case of manifolds admitting a $S o l$ structure is well understood. We follow Peter Scott ([96] Theorems 4.17 and 5.3).

Theorem 8.6. $M$ is a closed three-dimensional manifold admitting a geometric structure modelled on Sol if and only if $M$ is finitely covered by 
a torus bundle over $S^{1}$. Moreover, the identity component of Isom(Sol) intersects the fundamental group of $M$ in a subgroup $G \subset \pi_{1}(M)$ of finite index that is isomorphic to $\mathbb{Z} \times \mathbb{Z}$.

Clearly, Theorem 8.6 yields that if $M$ has the Preissmann property then it cannot admit a $S o l$ structure.

4) The following result is proved in the book of Peter Scott (Theorems $4.15,4.16$ and 5.3$)$ :

Theorem 8.7. $M$ is a closed three-dimensional manifold admitting a geometric structure modelled on one of $S^{3}, \mathbb{R}^{3}, S^{2} \times \mathbb{R}, \mathbb{H}^{2} \times \mathbb{R}, \widehat{S(2, \mathbb{R})}$ or $N i l$ if and only if $M$ is a Seifert fiber space. Moreover, if the geometric structure of $M$ is $\mathbb{H}^{2} \times \mathbb{R}$, then $M$ is finitely covered by a trivial fiber bundle $M_{0}=S \times S^{1}$, where $S$ is a compact surface of genus greater than one.

Theorem 8.7 implies that if $M$ has the Preissmann property, then it cannot admit a $\mathbb{H}^{2} \times \mathbb{R}$ structure. Indeed, since in this case $M$ is a Seifert fiber space we have that for every $p \in M$, the subgroup of $\pi_{1}(M, p)$ generated by the homotopy class of the fiber through $p$ is infinite cyclic, and it is not difficult to show that it is a normal subgroup of $\pi_{1}(M, p)$. The fact that $M$ is finitely covered by a trivial bundle implies that there exists a subgroup of finite index of the fiber subgroup which has nontrivial center in $\pi_{1}(M)$. Since $\pi_{1}(M)$ has no torsion, we get a subgroup of $\pi_{1}(M)$ isomorphic to $\mathbb{Z} \times \mathbb{Z}$, which contradicts the Preissmann property.

$5)$ We are left to consider the cases of $\widehat{S L(2, \mathbb{R})}$ and $\mathbb{H}^{3}$ structures. The next result tells us that a Seifert fiber space without conjugate points is almost a product manifold.

Proposition 8.5. Let $(M, g)$ be a closed three-dimensional manifold without conjugate points. If $M$ is a Seifert fiber space, then $(\tilde{M}, \tilde{g})$ is quasiisometric to a Riemannian product $S \times \mathbb{R}$.

Proof. We just give an outline of the proof since it shares many ideas with the proof of Lemma 8.9. As we mentioned in item (4), there is a nontrivial, infinite cyclic subgroup of $\pi_{1}(M, p)$ attached to the $S^{1}$-fibers of a Seifert fiber space, that is a normal subgroup of $\pi_{1}(M, p)$. This is the key observation which leads to the proof of the proposition.

Claim: Let $(M, g)$ be a compact manifold without conjugate points such that $\pi_{1}(M)$ has a nontrivial, infinite cyclic normal subgroup $G$. Let $\gamma_{\theta} \subset$ $\tilde{M}$ be a lift of a closed geodesic representing a minimal generator of $G$ and let $T: \tilde{M} \longrightarrow \tilde{M}$ be the corresponding covering isometry. Then the displacement of $T$ is constant in $\tilde{M}$, and $\tilde{M}$ is quasi-isometric to $H_{\theta}(0) \times \mathbb{R}$ (here, $H_{\theta}(0)$ is endowed with the restriction of the metric $\tilde{g}$ ). 
This statement is well known for manifolds with nonpositive curvature, where $\tilde{M}$ splits in fact in a Riemannian product. Let us show first the following statement:

There exists $P>0$ such that the displacement $d_{T}$ of $T$ satisfies $d_{T}(x) \leq P$ for every $x \in \tilde{M}$.

To see this, recall that an iterate $g\left(\gamma_{\theta}\right)$ by a covering isometry $g$ is an axis of the conjugate $g T g^{-1}$ of $T$. The isometry $T$ belongs to a representation of $\pi_{1}(M, p)$, for $p=\Pi\left(\gamma_{\theta}(0)\right)$, in the group of isometries of $\tilde{M}$. By hypothesis, the group generated by $T$ is normal in this representation of $\pi_{1}(M, p)$. This implies that given a covering isometry $g$ in $\pi_{1}(M, p)$, there exists $n \in \mathbb{Z}$ such that $g T g^{-1}=T^{n}$. Therefore, $g\left(\gamma_{\theta}\right)$ is an axis of $T^{n}$ and by Lemma 6.1 item (3) it is an axis of $T$ as well.

Let $\theta=(\tilde{p}, v), \tilde{p} \in \tilde{M})$ and let $D_{0}$ be a compact fundamental domain containing $\tilde{p}$. Given a fundamental domain $D$ of $M$ in $\tilde{M}$ there exists a covering isometry $g$ in the above representation of $\pi_{1}(M, p)$ such that $g\left(D_{0}\right)=D$ and hence, $g\left(\gamma_{\theta}\right) \cap D$ is nonempty. So we get that every point in $\tilde{M}$ is within a finite distance from an axis of $T$.

Now, recalling that the displacement $d_{T}$ of $T$ restricted to their axes is just the period of the closed geodesic $\Pi\left(\gamma_{\theta}\right)$, we get that every fundamental domain in $\tilde{M}$ has a point where $d_{T}$ is the period of $\Pi\left(\gamma_{\theta}\right)$. By the continuity of $T$ and the compactness of the fundamental domains, there exists a constant $P>0$ such that $d_{T}(q) \leq P$ for every $q \in \tilde{M}$. This implies that the displacement is constant: indeed, the above argument implies that $d_{T}$ attains its maximum value in $\tilde{M}$, but Lemma 6.1 tells us that the critical points of $d_{t}$ are only minimum points. Therefore, the maximum and minimum values of $d_{T}$ are equal which yields that $d_{T}$ is constant. This finishes the proof of the statement.

The above statement shows that there is a rigidity phenomenon associated to the presence of normal subgroups of the fundamental group. In fact, since every point of $\tilde{M}$ is critical for $d_{T}$, we have that $\tilde{M}$ is foliated by lifts of the geodesic $\gamma_{\theta}$, which are translated by $T$ and which are perpendicular to the foliation of horospheres $H_{\theta}(t), t \in \mathbb{R}$. The map $T$ acts isometrically in the foliation of horospheres: $H_{\theta}(t)$ is isometric to $H_{\theta}(t+n O)$ for every $n \in \mathbb{Z}$, where $O$ is the period of $\Pi\left(\gamma_{\theta}\right)$. From this fact it is not hard to show that all horospheres are quasi-isometric. Namely, there exists constant $K, C$ such that any two horosphreres $H_{\theta}\left(t_{1}\right), H_{\theta}\left(t_{2}\right)$ endowed with the restriction of the metric $\tilde{g}$ are $K, C$-quasi-isometric. Now, a little amount of work allows to conclude that $\tilde{M}$ is quasi-isometric to $H_{\theta}(0) \times \mathbb{R}$, where the factor $\mathbb{R}$ represents the axes of $T$.

Proposition 8.5 and Theorem 8.7 imply that $M$ cannot admit neither a $\widehat{S L(2, \mathbb{R})}$ structure or a $N i l$ structure, since none of them is quasi-isometric to a product. 
After (1), (2), (3) and (4) we conclude that the only possible geometric structure for $M$ under the assumptions of Theorem 8.4 is $\mathbb{H}^{3}$, thus finishing the proof of the statement.

\section{$5 \quad$ Further remarks and questions}

We would like to finish the survey with a list of questions and problems arising from the many subjects considered along the exposition.

We start with the notions of asymptoticity introduced in Chapter 4 . The asymptoticity axiom for manifolds without conjugate points introduced by Pesin in [75] is stronger than our notion of weak asymptoticity defined in Chapter 8, in fact, Pesin's asymptoticity axiom implies the weak asymptoticity property . It would be interesting to know if quasi-convexity implies Pesin's asymptoticity axiom.

Regarding the theory of Morse homoclinic geodesics developed in Chapters 7 and 8 , notice that the hypothesis of Proposition 8.1 just requires no conjugate points and its conclusion seems to be close to the conclusion of Proposition 8.2. This motivates us to guess that Proposition 8.2 would hold without the quasi-convexity hypothesis.

The question of knowing if the Preissmann property implies the Gromov hyperbolicity of the fundamental group of a compact manifold manifold without conjugate points remains a very difficult question of geometric group theory. We saw in Chapter 7 that the answer to this question is positive in compact, analytic manifolds with nonpositive curvature by the Bangert-Schröeder closing lemma for flats and Eberlein's characterization (in terms of the existence of flats) of non Gromov hyperbolic universal coverings of manifolds with nonpositive curvature [35]. In compact, three dimensional manifolds without conjugate points we have just pointed out the connections of this problem with Thurston's geometrization conjecture and Perelman's work. Certainly, the existence of a hyperbolic metric in the manifold is stronger than the Gromov hyperbolicity of the fundamental group, and we cannot expect to have the same result in higher dimensions.

Finally, we would like to observe that the connections between weak stability properties of the geodesic flow and the global geometry of the manifold might exist outside the context of manifolds without conjugate points. It is interesting to point out that the two dimensional sphere does not admit an expansive geodesic flow, since expansiveness in this case implies that the surface has no conjugate points. This was proved by Paternain [73]. We could ask for instance if there exists a topologically stable geodesic flow in 
the two sphere. Moreover, we do not know if the shadowing property can be satisfied by the geodesic flow of a metric in the two sphere. It seems to be that weak stability properties of the geodesic flow would imply strong restrictions in the global geometry of the manifold. We could guess based on the Anosov theory that the weak stability properties considered in the survey might imply that the fundamental group of the manifold is infinite. How close to expansive geodesic flows are flows satisfying one of our weak stability properties? This field is completely open, we do not have any hints or results about the subject. 


\section{Bibliography}

[1] Abdenour, F., Díaz, L.: Shadowing in the $C^{1}$ topology. Preprint, 2004.

[2] Anosov, D.: Geodesic flow on closed Riemannian manifolds of negative curvature. Tr. Mat. Inst. Steklova 90 (1967).

[3] Arnold, V. I.: Mathematical Methods of Classical Mechanics. Second Edition. Graduate Texts in Mathematics, 60. Springer-Verlag, New York, Berlin, Heidelberg.

[4] Ballmann, W.: Nonpositively curved manifolds of higher rank. Annals of Math. (1985) 122, 597-609.

[5] Ballmann, W.: Lectures on Spaces of Nonpositive Curvature. DMV Seminar, Band 25 (1995).

[6] Ballmann, W., Brin, M., Burns, K.: On surfaces with no conjugate points. J. Diff. Geom. 25 (1987), 249-273.

[7] Ballmann, W., Brin, M., Eberlein, P.: Structure of manifolds of nonpositive curvature, I. Annals of Math. (1985) 2, 122, 171-203.

[8] Ballmann, W., Gromov, M., Schröeder, V.: Manifolds of nonpositive curvature. Boston, Birkhauser, 1985.

[9] Bangert, V.: Mather sets for twist maps and geodesics on tori. Dynamics reported, 1 (1988), U. Kirchgraber, H. O. Walter Editors, John Wiley and Sons and B.G. Teubner.

[10] Bangert, V.: Minimal geodesics. Erg. Th. Dyn. Sys. (1989) 10, 263286 .

[11] Bangert, V., Schröeder, V.: Existence of flat tori in analytic manifolds of nonpositive curvature. Ann. Sci. Ec. Norm. Sup. 24 (1991) 605-634.

[12] Barbosa, J., Ruggiero, R.: Rigidity of surfaces whose geodesic flows preserve smooth foliations of codimension 1. Proceedings of the Amer. Math. Soc. 135 (2007) 507-515.

[13] Barbosa, J., Ruggiero, R.: Uniqueness of central foliations of geodesic flows in compact surfaces without conjugate points. To appear in Nonlinearity. 
[14] Besson, G.: Preuve de la conjecture de Poincaré en déformant la métrique par la courbure de Ricci, d'après G. Perelman. Astérisque 307 (2006). Société Mathématique de France.

[15] Birkhoff, G. D.: Surface transformations and their dynamical applications. Acta Math., 43 (1922), 1-119.

[16] Bonk, M.: Quasi-geodesic segments and Gromov hyperbolic spaces. Geom. Ded. (1996) 62, 281-298.

[17] Bonatti, C., Díaz, L.: Persistent non-hyperbolic transitive diffeomorphisms. Annals of Math. (1996) 2, 143, 357-396.

[18] Bowen, R.: Equilibrium states and ergodic theory of Anosov diffeomorphisms. Lecture Notes in Math. Springer, Berlin, 1975.

[19] Burns, K.: The flat theorem fails for surfaces with no conjugate points. Preprint (1990).

[20] Burns, K., Knieper, G.: Rigidity of surfaces with no conjugate points. J. of Diff. Geom. 34 (1991) 623-650.

[21] Burago, D., Ivanov, S.: Riemannian tori without conjugate points are flat. Geom. Funct. Anal. 4 (1994), n. 3, 259-269.

[22] Busemann, H.: The geometry of geodesics. New York, Academic Press, 1955.

[23] Cheeger, J., Ebin, D.: Comparison theorems in Riemannian geometry. North Holland, Amsterdam, 1975.

[24] Croke, C.: Volumes of balls in manifolds without conjugate points. Internat. J. Math. 3 (1992) no. 4, 455-467.

[25] Croke, C., Kleiner, B.: On tori without conjugate points. Invent. Math. 120 (1995) no. 2, 241-257.

[26] Croke, C., Schröeder, V.: The fundamental group of compact manifolds wihout conjugate points. Comm. Math. Helv. 61 (1986) 161-175.

[27] De La Harpe, P., Ghys, E.: Sur les groupes hyperboliques d'après M. Gromov. Progress in Mathematics, 83. Birkhauser. Zurich.

[28] De Melo, W.: Structural stability of diffeomorphisms on two manifolds. Invent. Math. 21 (1973) 233-246.

[29] Do Carmo, M.: Geometria Riemanniana. IMPA. Projeto Euclides, 1979.

[30] Donnay, V., Pugh, C.: Anosov geodesic flows for embedded surfaces. Astérisque 287 )2003) 61-70. Geometric methods in dynamics, II, volume in honor of Jacob Palis. De Melo, Viana, Yoccoz Ed. 
[31] Dubrovin, B. A., Fomenko, A. T., Novikov, S. P.: Modern geometric methods and applications, part I. The geometry of surfaces, transformation groups and fields. Graduate texts in mathematics 93, SpringerVerlag. New York 1992.

[32] Eberlein, P.: Geodesic flows in certain manifolds without conjugate points. Trans. Amer. Math. Soc. 167 (1972) 151-170.

[33] Eberlein, P.: When is a geodesic flow of Anosov type I. J. Diff. Geom. 8 (1973) 437-463.

[34] Eberlein, P.: Geodesic flows in manifolds of nonpositive curvature. Proceedings of Symposia in Pure Mathematics. Volume 69 (2001) 525571, AMS Providence, Rhode Island.

[35] Eberlein, P., O’Neil, B.: Visibility manifolds. Pacific J. Math. 46 (1973) 45-109.

[36] Epstein, D. B. A.: Periodic flows on 3-manifolds. Annals of Math. 95 (1972) 66-82.

[37] Eschenburg, J.-H.: Horospheres and the stable part of the geodesic flow. Math. Z. 153 (1977) 237-251.

[38] Franks, J.: Anosov diffeomorphisms on tori. Transactions of the AMS, 145 (1969) 117-124.

[39] Freedman, M. H. The Topology of Four-Differentiable Manifolds. J. Diff. Geom. 17 (1982) 357-453.

[40] Freire, A., Mañé, R.: On the entropy of the geodesic flow in manifolds without conjugate points. Inv. Math. 69 (1982) 375-392.

[41] Furstenberg, H.: The unique ergodicity of the horocycle flow. Recent advances in topological dynamics (Proc. Conf., Yale Univ., New Haven, Conn., 1972; in honor of Gustav Arnold Hedlund), pp. 95-115. Lecture Notes in Math., Vol. 318, Springer, Berlin, 1973.

[42] Ghys, E.: Flots d'Anosov sur les 3-variétés fibrées en cercles. Ergod. Th. Dynam. Sys. 4 (1984) 67-80.

[43] Ghys, E.: Flots d'Anosov dont les feuilletages stables sont différentiables. Ann. Scient. c. Norm. Sup. 20 (1987), 251-270.

[44] Green, L.: Geodesic instability. Proc. Amer. Math. Soc. 7 (1956) 438448.

[45] Green, L.: A theorem of E. Hopf. Michigan Math. Journal 5 (1958) 31-34.

[46] Gromov, M.: Hyperbolic groups. Essays in group theory 75-263. Gersten Ed. Springer-Verlag, New York.

[47] Gulliver, R.: On the variety of manifolds without conjugate points. Trans. Amer. Math. Soc. 210 (1975), 185-201. 
[48] Hasselblat, B., Katok, A.: Introduction to the modern theory of dynamical systems. Encyclopedia of Mathematics and its applications, vol. 54. G.-C. Rota Editor. Cambridge University Press, 1995.

[49] Hedlund, G.: Geodesics on a two dimensional Riemannian manifolds with periodic coefficients. Annals of Math. 33 (1932) 719-739.

[50] Hedlund, G.: Fuchsian groups and transitive horocycles. Duke Mathematical Journal II (1936) 530-542.

[51] Hirsch, M., Pugh, C., Shub, M.: Invariant manifolds. Vol. 583 of Lecture Notes in Mathematics, Springer-Verlag 1977.

[52] Hopf, E.: Statistik der geodätischen Linien in Mannigfaltigkeiten negativer Krümmung. Ber. Verh. Sächs. Akad. Wiss. Leipzig 91, (1939). 261-304.

[53] Hopf, E.: Closed surfaces without conjugate points. Proceedings of the National Academy of Sciences of the United States of America (1948), 47-51.

[54] Jaco, W., Shalen, P.: Seifert fibred spaces in 3-manifolds. Mem. Amer. Math. Soc. (1979) 220.

[55] Johannson, K.: Homotopy equivalences of 3-manifolds with boundary. Lecture Notes in Mathematics, 761, Springer, Berlin 1979.

[56] Kleiner, B., Lott, J.: Notes on Perelman's papers (2005). http://arxiv.org/abs/math.DG/0605667.

[57] Klingenberg, W.: Riemannian manifolds with geodesic flows of Anosov type. Ann. of Math. 99 (1974) 1-13.

[58] Klingenberg, W.: Lectures on closed geodesics. Springer, BerlinHeidelberg-New York, 1974.

[59] Kneser, H.: Gleschlossene Flächen in dreidimensionale Maningfaltigkeiten. Jahresber. Deutsch Math.-Verein 38 (1929) 248-260.

[60] Knieper, G.: Mannifaltigkeiten ohne konjugierte Punkte. Bonner Mathematische Schriften. 168 (1986).

[61] Knieper, G.: Hyperbolic dynamics and Riemannian geometry. Handbook of Dynamical systems, Vol. 1A. Hasselblat, B., Katok, A. Editors. (2002) Elsevier Science B.V.

[62] Lewowicz, J.: Expansive homeomorphisms of surfaces. Bol. Soc. Bras. Mat. (1989) 20, 113-133.

[63] Mañé, R.: An ergodic closing lemma. Ann. of Math. 116 (1982) 503540.

[64] Liao, S. T.: On the stability conjecture. Chinese Annals of Mathematics 1 (1980) 9-30. 
[65] Mañé, R.: A proof of the $C^{1}$ stability conjecture. Publications Mathématiques de l'IHES (1987) 66, 721-724.

[66] Mañé, R.: On a theorem of Klingenberg, In: M. I. Camacho, M. J. Pacífico, F. Takens (Ed.), Dynamical Systems and Bifurcation Theory, Longman Scientific \& Technical, New York, 1987, pp. 319-345.

[67] Mather, J.: Variational construction of connecting orbits. Ann. Inst. Fourier 43 (1993) 1349-1386.

[68] Milnor, J.: A unique factorization theorem for 3-manifolds. Amer. J. Math. 79 (1962) 1-7.

[69] Morse, M.: A fundamental class of geodesics on any closed surface of genus greater than one. Trans. Amer. Math. Soc. 26 (1924) 25-60.

[70] Moser, J.: Proof of a generalized form of a fixed point theorem due to G. D. Birkhoff. Lecture Notes in Math. Vol. 597. 464-549. Springer Verlag, Berlin, Heidelberg, New York 1977.

[71] Newhouse, S.: Quasi-elliptic periodic points in conservative dynamical systems. Amer. J. Math. 99 (1977), no. 5, 1061-1087.

[72] Paternain, G.: Geodesic flows. Progress in Mathematics, vol. 180, ISBN 0817641440, Boston, Birkhäusser, 1999.

[73] Paternain, M.: Expansive geodesic flows on surfaces. Ergod. Th. Dynam. Sys. 13 (1993) 153-165.

[74] Perelman, G. Ricci Flow with Surgery on Three-Manifolds. Preprint, March 2003.

[75] Pesin, Ya. B.: Geodesic flows on closed Riemannian manifolds without focal points. Math. USSR Izvestija. 11 (1977) 1195-1228.

[76] Preissmann, A.: Quelques propriétés globales des espaces de Riemann. Comm. Math. Helv. 15 (1943) 175-216.

[77] Pugh, C.: The closing lemma. Amer. J. Math. 89 (1967) 956-1009.

[78] Pugh, C., Shub, M.: The $\Omega$-stability theorem for flows. Amer. J. Math. 89 (1967) 1010-1021.

[79] Rauch, H. E.: A contribution to differential geometry in the large. Ann. of Math. 54 (1951) 38-55.

[80] Robbin, J. W.: A structural stability theorem. Annals of Mathematics (1971) 94, 447-493.

[81] Robinson, C.: Structural stability of $C^{1}$ diffeomorphisms. Journal of differential equations (1976) 22, 28-73.

[82] Rosas, V., Ruggiero, R.: On the Pesin set for expansive geodesic flows in manifolds without conjugate points. Bol. Soc. Bras. Mat. vol. 34, n. 2 (2003) 263-274. 
[83] Ruggiero, R.: On the creation of conjugate points. Math. Z. 208 (1991) 41-55.

[84] Ruggiero, R.: Expansive dynamics and Hyperbolic geometry. Bol. Soc. Bras. Mat. vol. 25, n. 2 (1994) 139-172.

[85] Ruggiero, R.: On a conjecture about expansive geodesic flows. Ergod. Th. Dynam. Sys. 16 (1996) 545-553.

[86] Ruggiero, R.: Expansive geodesic flows in manifolds with no conjugate points. Ergod. Th. Dynam. Sys. 17 (1997) 211-225.

[87] Ruggiero, R.: On nonhyperbolic quasiconvex spaces. Trans. Amer. Math. Soc. 350, 2 (1998) 665-687.

[88] Ruggiero, R.: Topological stability and Gromov hyperbolicity. Ergod. Th. Dynam. Sys. (1999), 19, 143-154.

[89] Ruggiero, R.: Weak stability of the geodesic flow and Preissmann's theorem. Ergod. Th. Dynam. Sys. (2000), 20, 1231-1251.

[90] Ruggiero, R.: On the nonexistence of rational geodesic foliations in the torus, Mather sets and Gromov hyperbolic spaces. Bol. Soc. Bras. Mat. (2000) 31, 1, 93-111.

[91] Ruggiero, R.: On the divergence of geodesic rays in manifolds without conjugate points, dynamics of the geodesic flow and global geometry. Astérisque 287 (2003) 231-250. Geometric methods in dynamics, II, volume in honor of Jacob Palis. De Melo, Viana, Yoccoz Ed.

[92] Ruggiero, R.: Shadowing of geodesics, weak stability of the geodesic flow and global hyperbolic geometry. Discrete and Continuous Dyn. Sys. Vol. 14, n. 2 (2006).

[93] Sakai, K.: Diffeomorphisms with weak shadowing. Fundamenta Math. 168 (2001) 1, 57-75.

[94] Sakai, K.: Diffeomorphisms with $C^{2}$ stable shadowing. Dyn. Sys. 17 (2002) 3, 235-241.

[95] Schröeder, V.: Codimension one tori in manifolds of nonpositive curvature. Geom. Dedicata (1990) 33, 251-263.

[96] Scott, P.: The geometries of 3-manifolds. Bull. London Math. Soc. 15 (1983) 401-487.

[97] Smale, S. Generalized Poincaré's Conjecture in Dimensions Greater than Four. Ann. Math. 74 (1961) 391-406.

[98] Stallings, J.: Polyhedral homotopy spheres. Bull. Amer. Math. Soc. 66 (1960) 485-488.

[99] Thurston, W.: Hyperbolic structures on three manifolds I. Annals of Math. 124 (1986) 203-246. 
[100] Thurston, W.: Hyperbolic structures on three manifolds II. Preprint (1987).

[101] Verjovsky, A.: Sistemas de Anosov. XII ELAM, Monografías del IMCA, Lima, Perú, 1999.

[102] Wolf, J. A.: Spaces of constant curvature. Mc Graw-Hill (1967).

[103] Walters, P.: On the pseudo-orbit tracing property and its relationship to stability. Lecture Notes in Mathematics, 608 (1978) 231-244.

[104] Zeeman, E. C. The Generalised Poincaré Conjecture. Bull. Amer. Math. Soc. 67, 270 (1961).

Rafael O. Ruggiero

Universidade Católica do Rio de Janeiro

Rua Marqués de São Vicente, 225, Gávea, 22453-900

Rio de Janeiro, Brazil

rorr@mat.puc-rio.br

www.mat.puc-rio.br 